\title{
A Severe Asthma Disease Signature from Gene Expression Profiling of Peripheral Blood from U- BIOPRED Cohorts
}

Jeannette Bigler ${ }^{1^{* \wedge}}$, Michael Boedigheimer ${ }^{2 \wedge}$, James P.R. Schofield ${ }^{3}$, Paul J. Skipp ${ }^{3}$, Julie Corfield $^{4}$, Anthony Rowe ${ }^{5}$, Ana R. Sousa ${ }^{6}$, Martin Timour ${ }^{1}$, Lori Twehues ${ }^{2}$, Xuguang Hu ${ }^{7}$, Graham Roberts $^{8}$, Andrew A. Welcher ${ }^{2}$, Wen Yu ${ }^{1 \#}$, Kian F. Chung ${ }^{9}$, lan M. Adcock ${ }^{9}$, Peter J. Sterk ${ }^{10}$, and Ratko Djukanović ${ }^{8}$ on behalf of The U-BIOPRED Study Group with input from the U-BIOPRED Patient Input Platform and patient representatives from the Ethics Board and Safety Management Board.

\section{Affiliations:}

${ }^{1}$ Amgen Inc, Seattle, WA

${ }^{2}$ Amgen Inc, Thousand Oaks, CA

${ }^{3}$ Centre for Biological Sciences, Southampton University, Southampton, United Kingdom

${ }^{4}$ AstraZeneca R\&D Molndal, Sweden and Areteva R\&D, Nottingham, UK

${ }^{5}$ Janssen Research and Development, High Wycombe, Buckinghamshire, United Kingdom

${ }^{6}$ Respiratory Therapeutic Unit, GSK, Stockley Park, UK

${ }^{7}$ Amgen Inc, South San Francisco, CA

${ }^{8}$ Respiratory Biomedical Research Unit, Faculty of Medicine, University Hospital Southampton, Southampton, UK

${ }^{9}$ National Heart \& Lung Institute, Imperial College \& Biomedical Research Unit, Royal Brompton \& Harefield NHS Trust, London, UK

${ }^{10}$ Dept. Respiratory Medicine, Academic Medical Centre, University of Amsterdam, The Netherlands

*Current address: BJ Group, LLC, Seattle, WA

\#Current address: Research Informatics, MedImmune LLC, Gaithersburg, MD

$\wedge$ These authors contributed equally to this work 
Corresponding Author:

Jeannette Bigler, PhD

BJ Group, LLC

3200 NE $92^{\text {nd }}$ Street

Seattle, WA 98115

E-mail: jeannette.bigler@bjgroupllc.com

\section{Author Contributions:}

Substantial contributions to the conception or design of the work: JB, MB, JPRS, PJS, JC, AR, ARS, GR, KFC, IMA, PJS, RD

The acquisition, analysis, or interpretation of data for the work: JB, MB, JPRS, PJS, MT, LT, XH, GR, AAW, WY, KFC, IMA, PJS, RD

Drafting the work or revising it critically for important intellectual content: JB, MB, AAW, KFC, IMA, PJS, RD

Final approval of the version to be published: all authors

Source of Support: U-BIOPRED is supported through an Innovative Medicines Initiative Joint Undertaking under grant agreement no. 115010, resources of which are composed of financial contribution from the European Union's Seventh Framework Programme (FP7/2007-2013) and EFPIA companies' in kind contribution (www.imi.europa.eu).

Running title: A Severe Asthma Gene Expression Signature in Blood

Descriptor number: 3.06 (Functional genomics/proteomics)

Word count body of manuscript: 3848 


\section{At a Glance Commentary}

Scientific Knowledge on the Subject

Asthma is a heterogeneous disease that only responds partially to currently available therapies.

Understanding heterogeneity in severe asthma at the molecular level and identifying biomarkers characterizing subgroups is essential to develop new, targeted therapies, and to select patients most likely to respond to these.

What this Study Adds to the Field

This study has detected marked differences in gene expression in blood cells between asthmatics and non-asthmatic individuals and produced a severe asthma disease signature composed of nearly 1700 genes. Based on gene expression, the population could be divided into two clusters: a cluster enriched for severe asthmatics (87\%), which included users of oral corticosteroid and individuals with blood neutrophilia, and a second cluster enriched for mixed severity asthmatic and non-asthmatic individuals. This study shows the value of blood transcriptomics for the identification of asthma stratification biomarkers and for the unbiased identification of molecular pathways of interest. Follow-up studies will be needed to take further the findings presented here.

This article has an online data supplement, which is accessible from this issue's table of content online at www.atsjournals.org 


\section{Abstract}

Rationale: Stratification of asthma at the molecular level, especially using accessible biospecimens, could greatly enable patient selection for targeted therapy.

Objectives: To determine the value of blood to identify transcriptional differences between clinically defined asthmatic and non-asthmatic groups, identify potential patient subgroups based on gene expression, and explore biological pathways associated with identified differences.

Methods: Transcriptomics profiles were generated by microarray analysis of blood from 610 asthmatic and control participants in U-BIOPRED. Differentially expressed genes (DEGs) were identified by ANOVA, including covariates for RNA quality, gender, and clinical site, and Ingenuity Pathway Analysis was applied. Patient subgroups based on DEGs were created by hierarchical clustering and topological data analysis.

Measurements and Main Results: 1693 genes were differentially expressed between severe asthmatics and non-asthmatics. The differences to non-asthmatics in non-smoking severe and mild/moderate asthmatics were significantly related $(r=0.76)$, with a larger effect size in the severe asthmatics. The majority, but not all, differences were explained by differences in circulating immune cell populations. Pathway analysis showed an increase in chemotaxis, migration, and myeloid cell trafficking in severe asthmatics, decreased B lymphocyte development and hematopoietic progenitor cells and lymphoid organ hypoplasia. Cluster 
analysis of DEGs created subgroups among the severe asthmatics that differed in molecular responses to oral corticosteroids.

Conclusions: Blood gene expression differences between clinically defined subgroups of asthmatics and non-asthmatic individuals as well as subgroups of severe asthma defined by transcript profiles show the value of blood in stratifying asthma patients and identifying molecular pathways for further study.

Word count abstract: 247

Keywords: biomarker, immune cell, microarray 


\section{Introduction}

Asthma is a complex disease that presents in various clinical forms and severity (1). While clinical severity is routinely used as an asthma classifier, it is not stable (2) and does not take into account the multiple underlying pathobiological processes. A substantial group of severe patients do not respond to existing therapies and have the greatest unmet needs $(1,3,4)$. With the exception of patients treated with the anti-IgE antibody, Omalizumab, the majority are treated with the same drugs despite heterogeneous underlying pathobiological mechanisms (5). Patient stratification efforts have used mainly clinical and pathophysiological parameters to understand underlying mechanisms (5). Recognizing the limitations of this approach to asthma, significant efforts are being made to stratify asthmatics using methods that bypass traditional clinical biases $(6,7)$.

Subgroups of asthma patients have been identified using either mechanistic, hypothesis-driven approaches (e.g. (8)) or unbiased statistical analyses of clinical and pathophysiological characteristics $(9,10)$. Additionally, molecular and genetic markers have been considered with the goal of understanding the pathobiological mechanisms underlying each subgroup (6). A subgroup of mild/moderate asthmatics expresses high levels of some Th2-associated genes in airway epithelial brushings and is characterized by eosinophilic inflammation, atopy, and a good clinical response to inhaled corticosteroids (ICS) (6). Although generally accepted biomarker(s) for the identification of T2 asthma do not exist yet, a biomarker set, composed of blood eosinophil counts, fractional exhaled nitric oxide (FeNO) and serum periostin levels, is emerging as a predictor of corticosteroid response (11). In contrast, patients who lack some of the T2 
features, the non-T2 asthmatics, tend to be less responsive to ICS (6). Understanding their disease, therefore, is important to develop effective treatments $(12,13)$.

The heterogeneity of severe asthma involves more than T2 gene expression $(14,15)$. The UBIOPRED (Unbiased BIOmarkers in Prediction of REspiratory Disease outcomes) study of severe asthma (16), obtained 'omics data from bronchial biopsies, bronchial and nasal brushings, sputum, blood, and urine (17). We report here the focused analysis of gene expression in blood. Blood is an important medium through which inflammatory and immune cells, as well as systemic treatment, reach the lungs. Analysis of this compartment can provide insight into pathobiological pathways associated with disease severity or other clinically relevant features. There were two main objectives. First, we sought to identify the major differences in blood transcript profiles and molecular pathways between non-asthmatic study participants and asthmatics stratified as severe and mild/moderate (3) and whether this was affected by treatment with oral corticosteroids (OCS). Our second aim was to use an unbiased approach to cluster patients on the basis of gene expression, independent of clinical parameters, hypothesizing that this would point to processes that transcend the standard clinical strata and management with current therapies.

Some of the results of these studies have been previously reported in the form of an abstract (18). 


\section{Material and Methods}

\section{Study population}

U-BIOPRED is a multi-center prospective cohort study, involving 16 clinical centers in 11

European countries. The adult part of the U-BIOPRED study consists of four cohorts (16): i) severe asthmatics/non-smoking (nsm) ( $n=311)$, ii) severe asthmatics/smoking (sm) ( $n=110)$, iii) mild/moderate asthmatics/nsm ( $n=88)$, and iv) non-asthmatics/nsm $(n=101)$ (see Supplemental Methods for more detail).

\section{Ethics Statement}

The study was conducted in accordance with the principles expressed in the Declaration of Helsinki. It was approved by the Institutional Review Boards of all the participating institutions and adhered to the standards set by the International Conference on Harmonization and Good Clinical Practice. All participants provided written informed consent. The study is registered under NCT01982162 on clinicaltrials.gov.

\section{Samples}

Blood samples were collected from 606 study participants (309 non-smoking severe asthmatics, 110 smoking severe asthmatics, 87 non-smoking mild/moderate asthmatics and 100 nonsmoking non-asthmatic individuals).

\section{Microarray Analysis}

RNA was isolated using the PAXgene Blood RNA kit (Qiagen, Valencia, CA) with on-column DNase treatment (Qiagen). RNA integrity was assessed using a Bioanalyzer 2100 (Agilent Technologies, Santa Clara, CA). Samples with RIN $\geq 6$ were processed for microarray as described 
(19) and hybridized onto Affymetrix HT HG-U133PM+ arrays (Affymetrix, Santa Clara, CA) using a GeneTitan ${ }^{\circledR}$ according to Affymetrix technical protocols. The microarray data are deposited in GEO under GSE69683.

\section{Data Analysis Sets}

After RNA and microarray quality control and exclusion of samples due to discrepancies with demographic data, the 498 samples available for analysis were randomized into training and validation sets (Supp Table 1).

\section{Statistical Analysis}

Data were normalized and $\log 2$ transformed prior to statistical analyses. Differentially expressed genes (DEGs) and gene signatures were identified using ANOVA including covariates for RIN, clinical site, and gender. When age was examined as a covariate in the main model, there were no significant gene expression changes associated with age. Initial comparisons were made in a training set and results were tested in a separate validation set (Supp Table 1). Both sets were then combined for further analysis, including clustering. Volcano plots show an area containing most null results $(99.99 \%)$, referred to as the patch of disbelief (PoD). Findings outside the PoD with a local false discovery rate (IFDR) of $\leq 0.05$ were considered significant (20, 21). Correlations were found with differential cell counts as well as OCS use and these variables were included as covariates in some analyses as described in the supplement.

To discover asthma sub-groups without a priori hypotheses, unbiased Hierarchical Clustering was performed on standardized data using Euclidean distance and Ward linkage (see 
Supplement). Ingenuity Pathway Analysis (IPA; QIAGEN, Redwood City, CA) was used for functional analysis of DEG sets and predictors of upstream regulators.

\section{Gene Correlation Network Analysis}

Gene expression correlation matrices were created using subsets of genes as described in the text. Gene modules were identified using scaled correlation matrices (22).

\section{Topological Data Analysis}

The transcriptomics data was clustered by topological data analysis (TDA) (23-27). TDA provides geometric representation of the relationships between patient data and variables in high dimensional datasets. TDA structures were generated using Ayasdi Cure with a norm correlation metric and two Neighborhood lenses (resolution 38, gain 3.4 ×, equalized).

For further details of data acquisition and analysis please see online supplement.

\section{Results}

\section{Study Population}

Demographic and clinical characteristics for the participants included in the gene expression analysis are shown in Table 1. Females were more highly represented in the severe asthma/non-smoking cohort and the median age in the mild/moderate asthma and nonasthmatic cohorts was lower. Approximately $40 \%$ of severe asthmatics reported using OCS. FeNO, a marker of inflammation in the lung, IgE, white blood cell counts (WBC), blood eosinophils, and neutrophils were elevated in all the asthma cohorts compared to the non- 
asthmatic cohort. The increase in WBC was highest in severe asthmatics using OCS and in severe asthmatic smokers regardless of OCS use (Supp Fig 1).

\section{Severe Asthma Disease Signature (SADS)}

Compared to non-asthmatic participants, both the non-smoking and smoking severe asthmatics had markedly different gene expression profiles (Fig 1A,B). Both severe asthmatic cohorts had a larger number of DEGs and larger fold-changes for some of these genes. Some of the DEGs were selective for particular immune cell subtypes and major differences in expression were observed in genes associated with eosinophils and neutrophils (higher in asthma) and B cells (lower in asthma) (Supp Table 2). There was good agreement between gene expression differences (severe asthma vs. non-asthmatic) discovered in the training when compared to the validation set ( $r=0.83$; Supp Fig 2).

By comparison, mild/moderate asthmatics showed fewer differences to non-asthmatic participants (Fig 1C). Most disease/non-asthmatic gene expression ratios in the severe and mild/moderate asthmatics trended in the same direction, even in cases where the differences were not statistically significant. While the differences relative to non-asthmatic participants were larger in the severe asthmatics than in mild/moderate asthmatics, there was a good correlation between the groups ( $r=0.76$; Supp Fig 3).

A severe asthma disease signature was developed using data from the combined data set. The collection of probe sets outside the PoD from the severe asthmatics vs. non-asthmatics comparison was designated the Severe Asthma Disease Signature (SADS); it contained 2695 
probe sets, corresponding to 1693 genes. The top DEGs in severe asthmatics ranked by significance are shown in Table 2 and the full list in Supp Table 3).

Gene expression differences between the severe asthmatics and non-asthmatic individuals could be due either to differences in cell populations or different activation states of the cells. To control for cell count effects, the statistical analysis was repeated with total white blood cell counts, percent monocytes, lymphocytes, neutrophils, and eosinophils as covariates in the analysis. This reduced the number of probe sets with differential expression from 2695 to 268 (Supp Fig 4; Supp Table 4).

Forty percent of the severe asthma patients were on OCS maintenance treatment, which can have an effect on transcriptional regulation. The steroid-inducible gene, FKBP5, was easily detected in the blood of the U-BIOPRED participants (Supp Fig 5) but levels were elevated only in a subset of asthmatics on OCS, while ICS did not appear to have an effect on blood FKBP5 transcript levels (Supp Fig 5).

When only severe asthmatics not using OCS were compared to non-asthmatic controls, the signature was reduced to 877 probe sets (Suppl Table 5), of which 774 were shared with the SADS. Thus, about $30 \%$ of the SADS was not due to OCS use. A direct comparison of severe asthmatics using and not using OCS showed distinct differences between the groups (Supp Fig 6). There were 1442 differentially expressed probe sets corresponding to 893 genes. B cellassociated genes had lower expression among OCS users and a small number of eosinophilassociated genes had higher expression in this group. 


\section{Hierarchical Clustering of the SADS}

Hierarchical clustering using the full dataset and the probe sets in the SADS showed two major patient clusters (Fig 2A). One of the patient clusters contained $>87 \%$ severe asthmatics (severe asthma-enriched cluster (SA-EC)), whereas the other contained approximately $56 \%$ severe asthmatics (mixed cluster (MC)) (Table 3). About $90 \%$ and $86 \%$ of the non-asthmatics and mild/moderate asthmatics, respectively, were assigned to the MC.

To test the robustness of this clustering, we generated severe asthmatic vs. non-asthmatic signatures separately in the training and the validation sets. For both data sets, their respective signatures generated two main clusters, one corresponding to the MC and the other to the SAEC. As in the full data set, $>87 \%$ of participants in the SA-EC were from the severe asthma cohort. The MC contained about $88 \%$ and $84 \%$ non-asthmatics and mild/moderate asthmatics, respectively.

The robustness of the clustering into SA-EC and $M C$ was tested further by using the signature gene list generated from the training set to cluster the validation set and vice versa. There was a significant overlap between this and the original classification (Fisher exact test $p=3 e-10$ ). The signature gene list from the validation set was then used to cluster the training set, again with significant overlap $(p=2 e-5)$.

\section{Topological Data Analysis of the SADS}

TDA clustering was first performed on the training and validation gene expression sets and then on the full data set, thereby creating three TDA networks. TDA reproduced the two clusters identified by hierarchical clustering, with good separation of the SA-EC and MC clusters. (Fig. 
2B). In all three networks, the participants identified as members of the SA-EC (presented as red nodes in Fig 2B) localized to the top part of the structure, while the MC participants (presented as the blue nodes in Fig 2B) localized to the bottom part. There was little mixing of the SA-EC and MC clusters and edges throughout the structures, supporting the classification by hierarchical clustering.

\section{Differences between Asthma Clusters}

The proportion of OCS users was larger in the SA-EC: 60/101 (two participants had no information on OCS usage) severe asthmatics in the SA-EC used OCS [59\%; $95 \% \mathrm{Cl}(50 \%-69 \%)]$ versus 39/131 in the $\mathrm{MC}$ [30\%; 95\% Cl (22\%-38\%)]. There were also differences in blood cell counts between the clusters with SA-EC having higher total white cell and neutrophils, but lower lymphocyte, counts (Supp Fig 7).

When overlaid onto the TDA structure, participants with high neutrophil counts, defined as $>60 \%$ of neutrophils in blood, were similarly distributed to patients from SA-EC (Figure $2 B-$ panel B). In the MC, lymphocyte counts were elevated in the OCS users compared to non-users, while in the SA-EC, neutrophil counts were elevated in OCS users and eosinophil counts trended lower (ANOVA $\mathrm{p}=0.07$ ). No other clinical or pathophysiological variables were clearly associated with the MC and SA-EC.

\section{Pathway Analysis}

Notable pathways upregulated in peripheral blood of severe asthmatics compared to nonasthmatic participants were chemotaxis, mobilization, migration, and infiltration of myeloid cells (Table 4). Gene sets similar to those affecting myeloid cells were involved in functions 
associated with decreased viral and bacterial infections. In severe asthmatics' peripheral blood, there also was a decrease in pathways related to the abundance of B-lymphocytes and hematopoietic progenitor cells, B-cell development, and hypoplasia of lymphoid organs.

Table 5 shows the five top upstream activators (positive activation scores) and inhibitors (negative activation scores). The upstream regulators include cytokines, enzymes, transcription factors, as well as fluticasone. As OCS use was a clinical variables and there were apparent differences in the effect of OCS use in the SA-EC and the MC, we looked for effects of OCS use on gene expression by cluster. In the MC, there were no gene expression differences between OCS users and non-users, whereas extensive differences were present in the SA-EC. Pathway analysis of the DEGs between OCS users and non-users in the SA-EC showed that these genes are known corticosteroids targets. The top three predicted upstream regulators were dexamethasone, fluticasone, and prednisolone (data not shown).

\section{Discussion}

This is the first comprehensive study of whole genome expression in circulating cells in asthma, a complex respiratory disease in which the contribution of circulating inflammatory cells is poorly understood. It is also the first study to explore the value of transcriptomic analysis of blood cells for asthma stratification. It shows that gene expression does not follow standard clinical classification that is routinely used in clinical practice and for drug development. Prespecified statistical analyses were first applied to data from cohorts of asthmatics defined by the U-BIOPRED consensus group (3) and from non-asthmatic participants. Marked differences between non-asthmatic individuals and severe asthmatics and fewer differences between 
mild/moderate asthmatics and non-asthmatics suggest a continuum of pathobiology from mild/moderate to severe disease. We then applied unbiased clustering, the principal objective of U-BIOPRED, which stratified the study participants into two main clusters: a SA-EC, that consisted mostly of severe asthmatics, and a MC, consisting of about $56 \%$ severe asthmatics. More than $85 \%$ of the enrolled non-asthmatics and mild/moderate asthmatics were in the MC.

In the pairwise comparisons between the clinically defined non-asthmatic and severe asthmatic cohorts, several DEGs expressed in specific immune cells stood out. Genes associated with Blymphocytes, including TCL1A, EBF1, TSPAN13, IGK@, BLNK, and FAM129C and genes associated with T-cells (IL6ST, LRRN3, and MAN1C1) $[(28,29)$ and unpublished Amgen data] had lower expression in severe asthmatics. Other down-regulated genes in severe asthma (AKAP2, STRBP, TCF4, BCL11A, RAB30, and CCDC50) have been reported as most highly expressed in Blymphocytes but are also present in other cell types $[(28,29)$ and unpublished Amgen data]. Genes mainly associated with granulocytes with some contributions from T-cells (e.g. DEF4A, OFLM4, CEACAM8, LTF, CCL23, or BPI) $[(28,29)$ and unpublished Amgen data] had higher expression in severe asthmatics. Recently, signatures of eosinophilic and granulocytic inflammatory signals in whole blood were found to be associated with lower asthma control (30). Of the six TREM-1/LPS signaling genes, CCL23, OLIG1 and OLIG2 were contained in the SADS.

The number of DEGs was reduced by about $90 \%$ when blood cell counts were included as covariates. The DEGs that persisted may indicate altered cell function, although we cannot exclude that additional cell types, for which we did not have frequency data, contributed to the 
SADS. After adjusting for cell counts, genes associated with B-lymphocytes and IPA functions such as lymphoid organ hypoplasia and decrease in B-lymphocyte development remained significant. While these differences were clearly detectable in the severe asthmatics, they were reduced or absent in the mild/moderate asthmatics. This observation is consistent with the notion that effects on B-lymphocytes are due either to severity of disease itself or OCS treatment in the severe asthmatics. Indeed, our analysis of severe OCS users and non-users showed a considerable number of DEGs. About 10 percent of the DEGs with lower expression in OCS users were B cell-selective. However, in this comparison, we cannot rule out contributions from disease, as OCS users by definition have more severe disease than OCS non-users. Furthermore, the number of probe sets in a signature derived from a comparison of severe asthmatics not using OCS with non-asthmatics was considerably smaller than the SADS. As in the SADS, B and T cell-selective genes had lower expression in the asthmatics and eosinophilselective genes had higher expression. In addition to the absence of OCS effects, better asthma control and, therefore, less severe disease and the smaller sample size likely contributed to this observation.

Consistent with the observed gene expression differences, the top upstream inhibitors included PAX5, TCF3, and MYC, all of which are transcriptional regulators with roles in B- and T-cell development, early B-cell differentiation, or lymphoid carcinogenesis $(31,32)$. The top upstream activators included genes involved in activation of granulocyte production, differentiation, and function (upstream regulators CSF3, NOS2, and CEBPE) (33-35). The identification of the topical (inhaled) corticosteroid, fluticasone, as an upstream activator confirmed the presence of steroid effects on gene expression. TGM2 is a cross-linking enzyme 
with a role in cell adhesion, wound healing, proliferation, and cellular motility (36). Its expression is increased in inflammatory and allergic conditions and there is evidence for a role of TGM2 in allergic asthma $(37,38)$. It is also the implicated auto-antigen in celiac disease $(39)$.

An important consideration for the observations made in this study is whether they reflect clinical severity of asthma or treatment. Severity is defined by symptoms, lung physiology, and the various types and doses of medications used for symptom control (5). Of these three elements, treatment with OCS potentially has the greatest impact on gene expression of circulating cells. OCS is, therefore, the most important confounder because of greater systemic bioavailability than ICS, which have a predominantly local anti-inflammatory effect (40). Furthermore, the inflammatory milieu within the lungs may influence gene expression in blood cells, partly because many of them re-circulate and partly because of systemic mediator signals from the lungs. Corticosteroids inhibit neutrophil apoptosis and contribute to neutrophil activation (41-43) and are likely to account, at least partly, for the observed blood neutrophilia. Corticosteroids also reduce eosinophil longevity (44). Furthermore, glucocorticoids induce cell death in lymphoid cells and are included in chemotherapy regimens for lymphoid malignancies (45). However, the doses used in asthma therapy may not be sufficient to kill lymphoid cells and no reduction in lymphoid cell count was observed. Therefore, corticosteroid effects on lymphocyte function are probably more relevant.

Corticosteroids act through specific receptors, including the nuclear receptor subfamily 3 (NR3C), which function as ligand-dependent transcriptional regulators (46). In the absence of robust published data exploring the effects of OCS on blood cell gene expression in a human 
population in vivo, FKBP5 expression was used to gauge the effects of asthma therapy. FKBP5 is an NRC3 chaperone, is highly expressed in blood of both non-asthmatics and asthmatics, and is itself corticosteroid-inducible (47). With the exception of one asthmatic, all the OCS users with elevated FKBP5 levels were in the SA-EC. The segregation of patients with elevated FKBP5 levels into the SA-EC, especially the non-smokers, suggests that disease severity is associated with high FKBP5 expression. Whether this is due to underlying disease mechanisms or treatment with OCS is unclear. Whether the high variability within each patient group reflects interindividual differences in the ability to respond to corticosteroids, possibly due to NR3C or FKBP5 genetic variation (48), the presence of oxidative/nitrosative stress (NOS2 above), or the lack of adherence to asthma treatment, requires further analysis.

This study creates a solid basis for further assessment of the clinical value of the observed DEGs. The two classes of patients distinguished by blood transcript profiling did not align in a simple way with clinical characteristics, so the clinical applicability has yet to be defined. Gene sets identifying the two clusters can be developed based on the data from this study and can be tested prospectively in observational and intervention studies. Observational studies would serve to validate the findings of this study while intervention studies could provide insight into appropriate courses of treatment for the two classes of patients.

Blood eosinophilia is now widely used as a stratification biomarker of so-called T2 type asthma (49-52). As the reporting of eosinophil counts can vary between laboratories in multi-center studies, the expression of several eosinophil-selective genes (ALOX15, ADORA3, or CCL23), found in this study to correlate with eosinophil counts, could be used for a more consistent 
measure of eosinophil numbers. However, blood transcript analysis cannot be used to stratify patients of other genes associated with T2 asthma: POSTN, CLCA1 and SERPINB2. These T2 genes were shown to be elevated in lung epithelial cells and in steroid-naive asthmatics (53) and to be responsive to IL-13 and corticosteroids $(53,54)$, but only SERPINB2 transcripts were detectable in blood in the current study, likely derived from myeloid cells. Furthermore, this transcript was not elevated in asthmatics and there was no indication of down-regulation in patients taking maintenance OCS. Thus, these genes appear to play a role in T2 high or low classification only in lung samples but not in blood.

One limitation of this study is that a number of clinical outcomes used in U-BIOPRED relied on self-reporting by the participants, which is subject to greater inaccuracy than measurements of physiologic or pathobiological biomarkers. For proper interpretation of blood transcript profiling data, it is important to have reliable information on OCS use. Non-adherence to treatment in severe asthma is estimated to be $30-70 \%$ (55). In U-BIOPRED, participants were required to have been under follow-up by a respiratory physician for at least six months, while their asthma control was optimized and medication adherence assessed using the Medication Adherence Report Scale (MARS) (16). The average MARS score among non-smoking severe asthmatics was 22.44, suggesting good adherence to treatment. However, self-reported adherence tends to overestimate actual adherence to treatment (56). Furthermore, some severe asthmatics show relative corticosteroid insensitivity (57), which was not assessed in this study. The SA-EC cluster of asthmatics included patients not taking OCS but they still had expression profiles that were more similar to severe asthmatics taking OCS in the SA-EC than the severe asthmatics in the MC cluster. 
In summary, this study has provided convincing evidence of differential gene expression in blood in patients with asthma. Our study shows, for the first time, major differences in the activity of circulating cells that do not follow currently applied clinical classification based on severity of asthma. The findings presented here are hypothesis-generating and clinical studies will be needed to determine the utility of stratifying markers derived from this study. Follow-up biological studies may lead to new insights into asthma disease mechanisms and open new avenues for therapeutic intervention. 


\section{Acknowledgments}

U-BIOPRED is supported through an Innovative Medicines Initiative Joint Undertaking under grant agreement no. 115010, resources of which are composed of financial contribution from the European Union's Seventh Framework Programme (FP7/2007-2013) and EFPIA companies' in kind contribution (www.imi.europa.eu). We thank all the members of each recruiting Centre (Appendix) for the recruitment and assessment of the participants. 


\section{References}

1. Bousquet J, Mantzouranis E, Cruz AA, Aït-Khaled N, Baena-Cagnani CE, Bleecker ER, Brightling CE, Burney P, Bush A, Busse WW, Casale TB, Chan-Yeung M, Chen R, Chowdhury B, Chung KF, Dahl R, Drazen JM, Fabbri LM, Holgate ST, Kauffmann F, Haahtela T, Khaltaev N, Kiley JP, Masjedi MR, Mohammad Y, O'Byrne P, Partridge MR, Rabe KF, Togias A, et al. Uniform definition of asthma severity, control, and exacerbations: Document presented for the World Health Organization Consultation on Severe Asthma. J Allergy Clin Immunol 2010;126:926-938.

2. Kupczyk M, Dahlén B, Sterk PJ, Nizankowska-Mogilnicka E, Papi A, Bel EH, Chanez P, Howarth PH, Holgate ST, Brusselle G, Siafakas NM, Gjomarkaj M, Dahlén SE. Stability of phenotypes defined by physiological variables and biomarkers in adults with asthma. Allergy Eur J Allergy Clin Immunol 2014;69:1198-1204.

3. Bel EH, Sousa A, Fleming L, Bush A, Chung KF, Versnel J, Wagener AH, Wagers SS, Sterk PJ, Compton $\mathrm{CH}$. Diagnosis and definition of severe refractory asthma: an international consensus statement from the Innovative Medicine Initiative (IMI). Thorax 2011;66:910917.

4. Chung KF, Wenzel SE, Brozek JL, Bush A, Castro M, Sterk PJ, Adcock IM, Bateman ED, Bel EH, Bleecker ER, Boulet L-P, Brightling C, Chanez P, Dahlen S-E, Djukanovic R, Frey U, Gaga M, Gibson P, Hamid Q, Jajour NN, Mauad T, Sorkness RL, Teague WG. International ERS/ATS guidelines on definition, evaluation and treatment of severe asthma. Eur Respir J 2014;43:343-373. 
5. Bateman ED, Hurd SS, Barnes PJ, Bousquet J, Drazen JM, FitzGerald M, Gibson P, Ohta K, O’Byrne P, Pedersen SE, Pizzichini E, Sullivan SD, Wenzel SE, Zar HJ. Global strategy for asthma management and prevention: GINA executive summary. Eur Respir J 2008;31:143-178.

6. Wenzel SE. Asthma phenotypes: the evolution from clinical to molecular approaches. Nat Med 2012;18:716-725.

7. Holgate ST. Stratified approaches to the treatment of asthma. Br J Clin Pharmacol 2013;76:277-291.

8. Holguin F, Comhair SAA, Hazen SL, Powers RW, Khatri SS, Bleecker ER, Busse WW, Calhoun WJ, Castro M, Fitzpatrick AM, Gaston B, Israel E, Jarjour NN, Moore WC, Peters SP, Teague WG, Chung KF, Erzurum SC, Wenzel SE. An association between Larginine/asymmetric dimethyl arginine balance, obesity, and the age of asthma onset phenotype. Am J Respir Crit Care Med 2013;187:153-159.

9. Wenzel S. Severe asthma: from characteristics to phenotypes to endotypes. Clin Exp Allergy 2012;42:650-658.

10. Moore WC, Hastie AT, Li X, Li H, Busse WW, Jarjour NN, Wenzel SE, Peters SP, Meyers DA, Bleecker ER. Sputum neutrophil counts are associated with more severe asthma phenotypes using cluster analysis. J Allergy Clin Immunol 2014;133:1557-1563.e5.

11. Heaney LG, Djukanovic R, Woodcock A, Walker S, Matthews JG, Pavord ID, Bradding P, Niven R, Brightling CE, Chaudhuri R, Arron JR, Choy DF, Cowan D, Mansur A, Menzies- 
Gow A, Adcock I, Chung KF, Corrigan C, Coyle P, Harrison T, Johnston S, Howarth P, Lordan J, Sabroe I, Bigler J, Smith D, Catley M, May R, Pierre L, et al. Research in progress: Medical Research Council United Kingdom Refractory Asthma Stratification Programme (RASP-UK). Thorax 2016;71:187-189.

12. Fajt ML, Wenzel SE. Asthma phenotypes and the use of biologic medications in asthma and allergic disease: The next steps toward personalized care. J Allergy Clin Immunol 2016;135:299-310.

13. Berry A, Busse WW, Lapraz JC, Hedayat KM, Pauly P, Frank R, Hargreaves R, CastroRodriguez JA, Holberg CJ, Wright AL, Martinez FD, Strimbu K, Tavel JA, Lyons TJ, Basu A, Brumme Z, Wang B, Nair K, Brumme C, Pierres C de, Reddy S, al. et, El-Sadr WM, Lundgren J, Neaton JD, Gordin F, Abrams D, Arduino RC, Group S for M of ATS, et al. Biomarkers in asthmatic patients: Has their time come to direct treatment? J Allergy Clin Immunol 2016;137:1317-1324.

14. Chung KF, Adcock IM. How variability in clinical phenotypes should guide research into disease mechanisms in asthma. Ann Am Thorac Soc 2013;10 Suppl:S109-17.

15. Cowan DC, Taylor DR, Peterson LE, Cowan JO, Palmay R, Williamson A, Hammel J, Erzurum SC, Hazen SL, Comhair SAA. Biomarker-based asthma phenotypes of corticosteroid response. J Allergy Clin Immunol 2015;135:877-883.e1.

16. Shaw DE, Sousa AR, Fowler SJ, Fleming L, Roberts G, Corfield J, Pandis I, Bansal AT, Bel EH, Auffray C, Compton CH, Bisgaard H, Bucchioni E, Caruso M, Chanez P, Dahlén B, 
Dahlen S-E, Dyson K, Frey U, Geiser T, Gerhardsson de Verdier M, Gibeon D, Guo Y-K, Hashimoto S, Hedlin G, Jeyasingham E, Hekking P-PW, Higenbottam T, Horváth I, et al. Clinical and inflammatory characteristics of the European U-BIOPRED adult severe asthma cohort. Eur Respir J 2015;46:1308-21.

17. Wheelock CE, Goss VM, Balgoma D, Nicholas B, Brandsma J, Skipp PJ, Snowden S, Burg D, D’Amico A, Horvath I, Chaiboonchoe A, Ahmed H, Ballereau S, Rossios C, Chung KF, Montuschi P, Fowler SJ, Adcock IM, Postle AD, Dahlen S-E, Rowe A, Sterk PJ, Auffray C, Djukanovic R. Application of 'omics technologies to biomarker discovery in inflammatory lung diseases. Eur Respir J 2013;42:802-825.

18. Bigler J, Boedigheimer M, Rowe A, Twehues L, Chung KF, Djukanovic R, Sousa AR, Corfield J, Adcock IM, Welcher A, Sterk PJ. Transcriptomic Fingerprints in Peripheral Blood from the Adult Cohort of the U-BIOPRED Study. Am J Respir Crit Care Med 2015;A2486.doi:doi:10.1164/ajrccm-conference.2015.191.1_MeetingAbstracts.A2486.

19. Bigler J, Rand HA, Kerkof K, Timour M, Russell CB. Cross-study homogeneity of psoriasis gene expression in skin across a large expression range. PLoS One 2013;8:e52242.

20. Efron B, Tibshirani R, Storey JD, Tusher V. Empirical Bayes Analysis of a Microarray Experiment. J Am Stat Assoc 2001;96:1151-1160.

21. Ploner A, Calza S, Gusnanto A, Pawitan Y. Multidimensional local false discovery rate for microarray studies. Bioinformatics 2006;22:556-565.

22. Yip AM, Horvath S. Gene network interconnectedness and the generalized topological 
overlap measure. BMC Bioinformatics 2007;8:22.

23. Hinks TSC, Zhou X, Staples KJ, Dimitrov BD, Manta A, Petrossian T, Lum PY, Smith CG, Ward J a., Howarth PH, Walls AF, Gadola SD, Djukanović R. Innate and adaptive T cells in asthmatic patients: Relationship to severity and disease mechanisms. J Allergy Clin Immunol 2015;1-11.doi:10.1016/j.jaci.2015.01.014.

24. Li L, Cheng W-Y, Glicksberg BS, Gottesman O, Tamler R, Chen R, Bottinger EP, Dudley JT. Identification of type 2 diabetes subgroups through topological analysis of patient similarity. Sci Transl Med 2015;7:311ra174-311ra174.

25. Nielson JL, Paquette J, Liu AW, Guandique CF, Tovar CA, Inoue T, Irvine K-A, Gensel JC, Kloke J, Petrossian TC, Lum PY, Carlsson GE, Manley GT, Young W, Beattie MS, Bresnahan JC, Ferguson AR. Topological data analysis for discovery in preclinical spinal cord injury and traumatic brain injury. Nat Commun 2015;6:8581.

26. Lum PY, Singh G, Lehman A, Ishkanov T, Vejdemo-Johansson M, Alagappan M, Carlsson J, Carlsson G. Extracting insights from the shape of complex data using topology. Sci Rep 2013;3:1236.

27. Carlsson G. Topology and data. Bull Am Math Soc 2009;46:255-308.

28. Allantaz F, Cheng DT, Bergauer T, Ravindran P, Rossier MF, Ebeling M, Badi L, Reis B, Bitter H, D’Asaro M, Chiappe A, Sridhar S, Pacheco GD, Burczynski ME, Hochstrasser D, Vonderscher J, Matthes T. Expression profiling of human immune cell subsets identifies miRNA-mRNA regulatory relationships correlated with cell type specific expression. PLoS 
One 2012;7:e29979.

29. Abbas AR, Baldwin D, Ma Y, Ouyang W, Gurney A, Martin F, Fong S, van Lookeren Campagne M, Godowski P, Williams PM, Chan AC, Clark HF. Immune response in silico (IRIS): immune-specific genes identified from a compendium of microarray expression data. Genes Immun 2005;6:319-331.

30. Croteau-Chonka DC, Qiu W, Martinez FD, Strunk RC, Lemanske, Jr. RF, Liu AH, Gilliland FD, Millstein J, Gauderman WJ, Ober C, Krishnan JA, White SR, Naureckas ET, Nicolae DL, Barnes KC, London SJ, Barraza-Villarreal A, Carey VJ, Weiss ST, Raby BA, Consortium on behalf of the AB for IGE (Asthma B. Gene Expression Profiling in Blood Provides Reproducible Molecular Insights into Asthma Control. Am J Respir Crit Care Med 2016;rccm.201601-01070C.doi:10.1164/rccm.201601-01070C.

31. Somasundaram R, Prasad MAJ, Ungerbäck J, Sigvardsson M. Transcription factor networks in B-cell differentiation link development to acute lymphoid leukemia. Blood 2015;126:144-152.

32. Nie Z, Hu G, Wei G, Cui K, Yamane A, Resch W, Wang R, Green DR, Tessarollo L, Casellas R, Zhao K, Levens D. C-Myc is a universal amplifier of expressed genes in lymphocytes and embryonic stem cells. Cell 2013;151:68-79.

33. Panopoulos AD, Watowich SS. Granulocyte colony-stimulating factor: molecular mechanisms of action during steady state and "emergency" hematopoiesis. Cytokine 2008;42:277-88. 
34. Kolaczkowska E, Kubes P. Neutrophil recruitment and function in health and inflammation. Nat Rev Immunol 2013;13:159-75.

35. Pabst T, Mueller BU. Complexity of CEBPA dysregulation in human acute myeloid leukemia. Clin Cancer Res 2009;15:5303-5307.

36. Griffin M, Casadio R, Bergamini CM. Transglutaminases : Nature's biological glues. Biochem J 2002;368:377-396.

37. Hallstrand TS, Wurfel MM, Lai Y, Ni Z, Gelb MH, Altemeier WA, Beyer RP, Aitken ML, Henderson WR. Transglutaminase 2, a novel regulator of eicosanoid production in asthma revealed by genome-wide expression profiling of distinct asthma phenotypes. PLoS One 2010;5:e8583.

38. Kim DY, Park BS, Hong GU, Lee BJ, Park JW, Kim SY, Ro JY. Anti-inflammatory effects of the R2 peptide, an inhibitor of transglutaminase 2 , in a mouse model of allergic asthma, induced by ovalbumin. Br J Pharmacol 2011;162:210-225.

39. Di Sabatino A, Vanoli A, Giuffrida P, Luinetti O, Solcia E, Corazza GR. The function of tissue transglutaminase in celiac disease. Autoimmun Rev 2012;11:746-753.

40. Derendorf H, Daley-Yates PT, Pierre LN, Efthimiou J. Systemic bioavailability of inhaled steroids: the importance of appropriate and comparable methodology. Eur Respir J Off J Eur Soc Clin Respir Physiol 2001;17:157-8.

41. Schleimer RP, Freeland HS, Peters SP, Brown KE, Derse CP. An assessment of the effects of glucocorticoids on degranulation, chemotaxis, binding to vascular endothelium and 
formation of leukotriene B4 by purified human neutrophils. J Pharmacol Exp Ther 1989;250:598-605.

42. Kato T, Takeda Y, Nakada T, Sendo F. Inhibition by dexamethasone of human neutrophil apoptosis in vitro. Nat Immun 1995;14:198-208.

43. Nguyen LT, Lim S, Oates T, Chung KF. Increase in airway neutrophils after oral but not inhaled corticosteroid therapy in mild asthma. Respir Med 2005;99:200-7.

44. Jones CP, Paula Neto HA, Assreuy J, Vargaftig BB, Gaspar Elsas MI, Elsas PX. Prostaglandin E2 and dexamethasone regulate eosinophil differentiation and survival through a nitric oxide- and CD95-dependent pathway. Nitric Oxide 2004;11:184-93.

45. Schmidt S, Rainer J, Ploner C, Presul E, Riml S, Kofler R. Glucocorticoid-induced apoptosis and glucocorticoid resistance: molecular mechanisms and clinical relevance. Cell Death Differ 11:S45-S55.

46. Lu NZ, Wardell SE, Burnstein KL, Defranco D, Fuller PJ, Giguere V. International Union of Pharmacology. LXV. The pharmacology and classification of the nuclear receptor superfamily: glucocorticoid, mineralcorticoid, progesterone, and androgen receptors. Pharmacol Rev 2006;58:782-797.

47. Jääskeläinen $\mathrm{T}$, Makkonen $\mathrm{H}$, Palvimo JJ. Steroid up-regulation of FKBP51 and its role in hormone signaling. Curr Opin Pharmacol 2011;11:326-31.

48. Koper JW, van Rossum EFC, van den Akker ELT. Glucocorticoid receptor polymorphisms and haplotypes and their expression in health and disease. Steroids 2014;92:62-73. 
49. Wenzel S, Ford L, Pearlman D, Spector S, Sher L, Skobieranda F, Wang L, Kirkesseli S, Rocklin R, Bock B, Hamilton J, Ming JE, Radin A, Stahl N, Yancopoulos GD, Graham N, Pirozzi G. Dupilumab in persistent asthma with elevated eosinophil levels. N Engl J Med $2013 ; 368: 2455-2466$.

50. Castro M, Wenzel SE, Bleecker ER, Pizzichini E, Kuna P, Busse WW, Gossage DL, Ward CK, Wu Y, Wang B, Khatry DB, van der Merwe R, Kolbeck R, Molfino NA, Raible DG.

Benralizumab, an anti-interleukin 5 receptor $\alpha$ monoclonal antibody, versus placebo for uncontrolled eosinophilic asthma: a phase $2 \mathrm{~b}$ randomised dose-ranging study. Lancet Respir Med 2014;2:879-90.

51. Castro M, Zangrilli J, Wechsler ME, Bateman ED, Brusselle GG, Bardin P, Murphy K, Maspero JF, O’Brien C, Korn S. Reslizumab for inadequately controlled asthma with elevated blood eosinophil counts: results from two multicentre, parallel, double-blind, randomised, placebo-controlled, phase 3 trials. Lancet Respir Med 2015;3:355-66.

52. Ortega HG, Liu MC, Pavord ID, Brusselle GG, FitzGerald JM, Chetta A, Humbert M, Katz LE, Keene ON, Yancey SW, Chanez P. Mepolizumab Treatment in Patients with Severe Eosinophilic Asthma. N Engl J Med 2014;371:1198-1207.

53. Woodruff PG, Modrek B, Choy DF, Jia G, Abbas AR, Ellwanger A, Arron JR, Koth LL, Fahy J V. T-helper type 2-driven inflammation defines major subphenotypes of asthma. Am J Respir Crit Care Med 2009;180:388-395.

54. Woodruff PG, Boushey HA, Dolganov GM, Barker CS, Yang YH, Donnelly S, Ellwanger A, 
Sidhu SS, Dao-Pick TP, Pantoja C, Erle DJ, Yamamoto KR, Fahy J V. Genome-wide profiling identifies epithelial cell genes associated with asthma and with treatment response to corticosteroids. Proc Natl Acad Sci U S A 2007;104:15858-63.

55. Lindsay JT, Heaney LG. Non-adherence in difficult asthma and advances in detection. Expert Rev Respir Med 2013;7:607-14.

56. Foster J, Lavoie K, Boulet L-P. Treatment adherence and psychosocial factors in severe asthma. In: Chung KF, Bel EH WS, editor. Eur Respir Monogr 51 Difficult-to-treat asthma Sheffield, UK: European Respiratory Society; 2011. p. 28-49.

57. Hew M, Bhavsar P, Torrego A, Meah S, Khorasani N, Barnes PJ, Adcock I, Chung KF. Relative corticosteroid insensitivity of peripheral blood mononuclear cells in severe asthma. Am J Respir Crit Care Med 2006;174:134-41. 


\section{Figure Legends}

Figure 1. Comparison of Non-smoking Mild/moderate Asthmatics, and Non-smoking and Smoking Severe Asthmatics with Non-smoking Non-asthmatic Volunteers. A. Severe asthmatics, nsm, B. Severe asthmatics, sm and C. Mild/moderate asthmatics. A positive fold-change indicates higher expression in asthmatics compared to non-asthmatic individuals and negative fold-change lower expression. Differentially expressed genes that were not assigned a cell type are ubiquitously expressed in immune cells. Light blue shaded area: Patch of Disbelief (nonsignificant differences).

Figure 2. A. Two-dimensional Clustering of the Severe Asthma Disease Signature. The heat map was generated using all the samples in the study and the genes that were differentially expressed between non-asthmatic individuals and severe asthmatics. Turquoise: class enriched in severe asthmatics; red: mixed class containing $90 \%$ of the non-asthmatic participants and $86 \%$ of the mild/moderate asthmatics. Blue squares: non-smoking severe asthmatics; turquoise squares: smoking severe asthmatics; brown squares: mild/moderate asthmatics; red squares: non-asthmatic individuals. B. Topological Data Analysis. Left: The distribution of classes derived from hierarchical clustering of the SADS in a network created by TDA using data from (top) the training set $(n=328)$, (middle) the validation set $(n=170)$, and (bottom) the whole data set $(n=$ 498). Red and blue nodes represent participants identified by hierarchical clustering as being within the severe asthma-enriched cluster and the mixed cluster, respectively. Right: The distribution of study participants with high neutrophil cell counts in TDA structures: red nodes represent those with blood neutrophil cell count $>60 \%$, and blue nodes represent those with 
blood neutrophil cell count $<60 \%$. [Metric: Norm Correlation. Lenses: Neighborhood Lens 1

(Res. 38, Gain 3.4), Neighborhood Lens 2 (Res. 38, Gain 3.4)]. 


\section{Table 1 Demographic Characteristics}

\begin{tabular}{|c|c|c|c|c|}
\hline Characteristic & $\begin{array}{c}\text { Severe } \\
\text { Asthma/Non- } \\
\text { smoking }(n=246)\end{array}$ & $\begin{array}{c}\text { Severe } \\
\text { Asthma/Smoking } \\
(n=88)\end{array}$ & $\begin{array}{l}\text { Mild/moderate } \\
\text { Asthma/Non- } \\
\text { smoking }(n=77)\end{array}$ & $\begin{array}{c}\text { Non- } \\
\text { asthmatic/Non- } \\
\text { smoking }(n=87)\end{array}$ \\
\hline \multicolumn{5}{|l|}{ Gender } \\
\hline Male (n/\%) & $85 / 34.6$ & $45 / 51.1$ & $40 / 51.9$ & $53 / 60.9$ \\
\hline Female (n/\%) & $161 / 65.4$ & $43 / 48.9$ & $37 / 48.1$ & $34 / 39.1$ \\
\hline Age (years)* & $53(43 ; 62)$ & $55(48 ; 61)$ & $39(27 ; 55)$ & $37(27 ; 49)$ \\
\hline White Caucasian (\%) & 87.4 & 95.5 & 93.5 & 92.0 \\
\hline $\begin{array}{l}\text { OCS dose normalized to } \\
\text { prednisolone }(\mathrm{mg})^{*} \#\end{array}$ & $10(5.8 ; 16.3)$ & $10(7.5 ; 18.8)$ & NA & NA \\
\hline $\begin{array}{l}\text { Atopy } \\
\text { (positive/negative/unavailable) }\end{array}$ & $180 / 47 / 19$ & $54 / 27 / 7$ & $68 / 8 / 1$ & $32 / 44 / 11$ \\
\hline FEV1 \% predicted* & $67(50 ; 84)$ & $65(53 ; 75)$ & $92(77 ; 102)$ & $103(94 ; 110)$ \\
\hline FeNO ppb* & $26(15.5 ; 48.9)$ & $25(11.5 ; 48)$ & $25.5(18.4 ; 45.4)$ & $19(13.8 ; 26.8)$ \\
\hline $\operatorname{lgE} \mathrm{IU} /\left.\mathrm{m}\right|^{*}$ & $112(44 ; 317)$ & $140(70 ; 378)$ & $102(53 ; 244)$ & $27(9 ; 68)$ \\
\hline Blood eosinophils $10^{3} / \mu \mathrm{L}^{*}$ & $0.20(0.10 ; 0.40)$ & $0.23(0.12 ; 0.40)$ & $0.20(0.10 ; 0.30)$ & $0.10(0.10 ; 0.20)$ \\
\hline Blood neutrophils $10^{3} / \mu \mathrm{L}^{*}$ & $4.7(3.5 ; 6.1)$ & $4.8(3.8 ; 6.6)$ & $3.3(2.7 ; 4.5)$ & $3.0(2.4 ; 3.9)$ \\
\hline Blood lymphocytes $10^{3} / \mu \mathrm{L}^{*}$ & $1.9(1.5 ; 2.4)$ & $2.1(1.6 ; 2.6)$ & $1.7(1.5 ; 2.1)$ & $1.7(1.4 ; 2.2)$ \\
\hline Total white blood cells $10^{3} / \mu \mathrm{L}^{*}$ & $7.8(6.2 ; 9.4)$ & $7.9(6.7 ; 9.8)$ & $5.8(5.1 ; 7.2)$ & $5.3(4.7 ; 6.4)$ \\
\hline *median (interquartile range) & & & & \\
\hline
\end{tabular}


Table 2 Genes with Most Significant Differential Expression between Severe Asthmatics and Non-asthmatic Individuals

\begin{tabular}{cccc}
\multicolumn{4}{c}{ Higher Expression in Severe Asthmatics } \\
Gene symbol & $\begin{array}{c}\text { Fold } \\
\text { Change }\end{array}$ & P-value & IFDR \\
DEFA4 & 2.58 & $7.13 \mathrm{E}-10$ & 0.00 \\
OLFM4 & 2.43 & $2.27 \mathrm{E}-07$ & 0.00 \\
CEACAM8 & 2.40 & $2.17 \mathrm{E}-09$ & 0.00 \\
LTF & 2.20 & $5.79 \mathrm{E}-09$ & 0.00 \\
MMP8 & 2.18 & $9.98 \mathrm{E}-09$ & 0.00 \\
BPI & 2.03 & $3.57 \mathrm{E}-09$ & 0.00 \\
LCN2 & 2.03 & $5.45 \mathrm{E}-09$ & 0.00 \\
CRISP3 & 1.98 & $8.07 \mathrm{E}-09$ & 0.00 \\
RNASE3 & 1.98 & $2.65 \mathrm{E}-08$ & 0.00 \\
CEACAM6 & 1.97 & $8.44 \mathrm{E}-09$ & 0.00 \\
CCL23 & 1.86 & $3.52 \mathrm{E}-06$ & 0.00 \\
HP & 1.75 & $2.52 \mathrm{E}-09$ & 0.00 \\
DEFA1 & 1.72 & $3.04 \mathrm{E}-10$ & 0.00 \\
MS4A3 & 1.69 & $1.09 \mathrm{E}-06$ & 0.00 \\
CTSG & 1.68 & $5.90 \mathrm{E}-07$ & 0.00 \\
CD24 & 1.62 & $5.34 \mathrm{E}-08$ & 0.00 \\
ANXA3 & 1.60 & $2.33 \mathrm{E}-08$ & 0.00 \\
SLPI & 1.60 & $2.16 \mathrm{E}-09$ & 0.00 \\
CAMP & 1.58 & $6.95 \mathrm{E}-09$ & 0.00 \\
RNASE2 & 1.56 & $1.57 \mathrm{E}-07$ & 0.00 \\
TCN1 & 1.52 & $7.10 \mathrm{E}-08$ & 0.00 \\
F5 & 1.47 & $1.21 \mathrm{E}-10$ & 0.00 \\
CEBPE & 1.46 & $6.86 \mathrm{E}-09$ & 0.00 \\
GAPT & 1.36 & $4.56 \mathrm{E}-10$ & 0.00 \\
PNPLA1 & 1.35 & $1.40 \mathrm{E}-08$ & 0.00 \\
STXBP5 & 1.31 & $1.04 \mathrm{E}-08$ & 0.00 \\
ACSL1 & 1.30 & $6.85 \mathrm{E}-09$ & 0.00 \\
ALAS1 & 1.21 & $1.46 \mathrm{E}-09$ & 0.00 \\
AMPD3 & 1.19 & $8.84 \mathrm{E}-10$ & 0.00 \\
MYD88 & 1.18 & $1.70 \mathrm{E}-10$ & 0.00
\end{tabular}

\begin{tabular}{cccc}
\multicolumn{4}{c}{ Lower Expression in Severe Asthmatics } \\
& Fold & P-value & IFDR \\
Gene symbol & Change & & \\
TCL1A & -1.71 & $9.51 \mathrm{E}-07$ & 0.00 \\
EBF1 & -1.69 & $4.56 \mathrm{E}-09$ & 0.00 \\
TSPAN13 & -1.69 & $1.91 \mathrm{E}-12$ & 0.00 \\
IGK@ & -1.68 & $1.31 \mathrm{E}-09$ & 0.00 \\
LRRN3 & -1.66 & $4.83 \mathrm{E}-07$ & 0.00 \\
CUX2 & -1.49 & $2.63 \mathrm{E}-09$ & 0.00 \\
AFF3 & -1.47 & $8.43 \mathrm{E}-08$ & 0.00 \\
BLNK & -1.46 & $2.89 \mathrm{E}-09$ & 0.00 \\
AKAP2 & -1.44 & $1.96 \mathrm{E}-08$ & 0.00 \\
STRBP & -1.43 & $1.83 \mathrm{E}-09$ & 0.00 \\
IL6ST & -1.42 & $2.30 \mathrm{E}-09$ & 0.00 \\
MAN1C1 & -1.40 & $1.59 \mathrm{E}-08$ & 0.00 \\
FAM129C & -1.39 & $3.69 \mathrm{E}-08$ & 0.00 \\
TCF4 & -1.39 & $5.04 \mathrm{E}-11$ & 0.00 \\
TTN & -1.38 & $5.81 \mathrm{E}-09$ & 0.00 \\
CCR6 & -1.37 & $3.00 \mathrm{E}-09$ & 0.00 \\
RAB11FIP3 & -1.37 & $1.20 \mathrm{E}-09$ & 0.00 \\
BCL11A & -1.36 & $2.99 \mathrm{E}-09$ & 0.00 \\
GPM6B & -1.36 & $8.25 \mathrm{E}-10$ & 0.00 \\
RAB30 & -1.35 & $7.20 \mathrm{E}-09$ & 0.00 \\
PDE7A & -1.34 & $1.41 \mathrm{E}-10$ & 0.00 \\
SLFNL1 & -1.31 & $6.97 \mathrm{E}-09$ & 0.00 \\
CCDC50 & -1.29 & $3.15 \mathrm{E}-09$ & 0.00 \\
ENAM & -1.26 & $3.80 \mathrm{E}-09$ & 0.00 \\
LUC7L & -1.25 & $9.86 \mathrm{E}-10$ & 0.00 \\
NSUN6 & -1.25 & $2.79 \mathrm{E}-09$ & 0.00 \\
TGIF2 & -1.25 & $7.67 \mathrm{E}-10$ & 0.00 \\
ZBTB20 & -1.25 & $1.17 \mathrm{E}-10$ & 0.00 \\
CBFA2T2 & -1.22 & $1.30 \mathrm{E}-09$ & 0.00 \\
KIAA0355 & -1.20 & $3.76 \mathrm{E}-10$ & 0.00
\end{tabular}


Table 3 Representation of the Four Cohorts in the Two Transcript Classes

\section{Cohort}

Non-asthmatic, nsm

Mild/moderate asthma, nsm

Severe asthma, nsm

Severe asthma, sm

Total

\section{Cluster Classification n (\%)}

Mixed (MC)

$78(22.8)$

$66(19.3)$

$143(41.8)$

55 (16.1)

$342(100)$
Severe Asthma

Enriched (SA-EC)

$9(5.8)$

$11(7.1)$

103 (66.0)

$33(21.2)$

$156(100)$ 
Table 4 Ingenuity Pathway Functional Analysis

\section{Summary of Functions}

Total Genes

Increased chemotaxis, recruitment and infiltration of

myeloid cells such as neutrophils; increased angiogenesis;

arthritis

Increase in protein metabolism and decrease in cancer

Decreased quantity and differentiation of lymphocytes and mononuclear leukocytes

Decreased viral infection

Increased apoptosis and decreased cell survival

Increased migration of mononuclear leukocytes and killing of bacteria; decrease in infections including fungal

infections

Hypoplasia of lymphoid organs and decreased quantity of hematopoietic progenitor cells; increase in quantity of double negative T-cells

Decreased development of pre-B, pro-B and B lymphocytes 


\section{Table 5 Ingenuity Pathway Analysis Top Upstream Regulators and Inhibitors}

$\begin{array}{llll}\begin{array}{l}\text { Upstream } \\ \text { Regulator }\end{array} & \text { Molecule Type } & \begin{array}{l}\text { p-value } \\ \text { of } \\ \text { Overlap }\end{array} & \begin{array}{l}\text { Activation } \\ \text { z-score }\end{array} \\ \text { CSF3 } & \text { Cytokine } & 9.18 \mathrm{E}-07 & 4.47 \\ \text { TGM2 } & \text { Enzyme } & 4.09 \mathrm{E}-08 & 4.08 \\ \text { NOS2 } & \text { Enzyme } & 1.00 \mathrm{E}+00 & 3.52 \\ \text { Fluticasone } & \text { Chemical drug } & 1.19 \mathrm{E}-05 & 3.38 \\ \text { CEBPE } & \text { Transcription regulator } & 4.58 \mathrm{E}-04 & 3.11 \\ \text { PAX5 } & \text { Transcription regulator } & 2.94 \mathrm{E}-06 & -2.76 \\ \text { TCF3 } & \text { Transcription regulator } & 1.41 \mathrm{E}-01 & -2.80 \\ \text { Immunoglobulin } & \text { Complex } & 1.10 \mathrm{E}-05 & -3.29 \\ \text { MKL2 } & \text { Transcription regulator } & 1.73 \mathrm{E}-03 & -3.32 \\ \text { MYC } & \text { Transcription regulator } & 1.15 \mathrm{E}-04 & -4.83\end{array}$



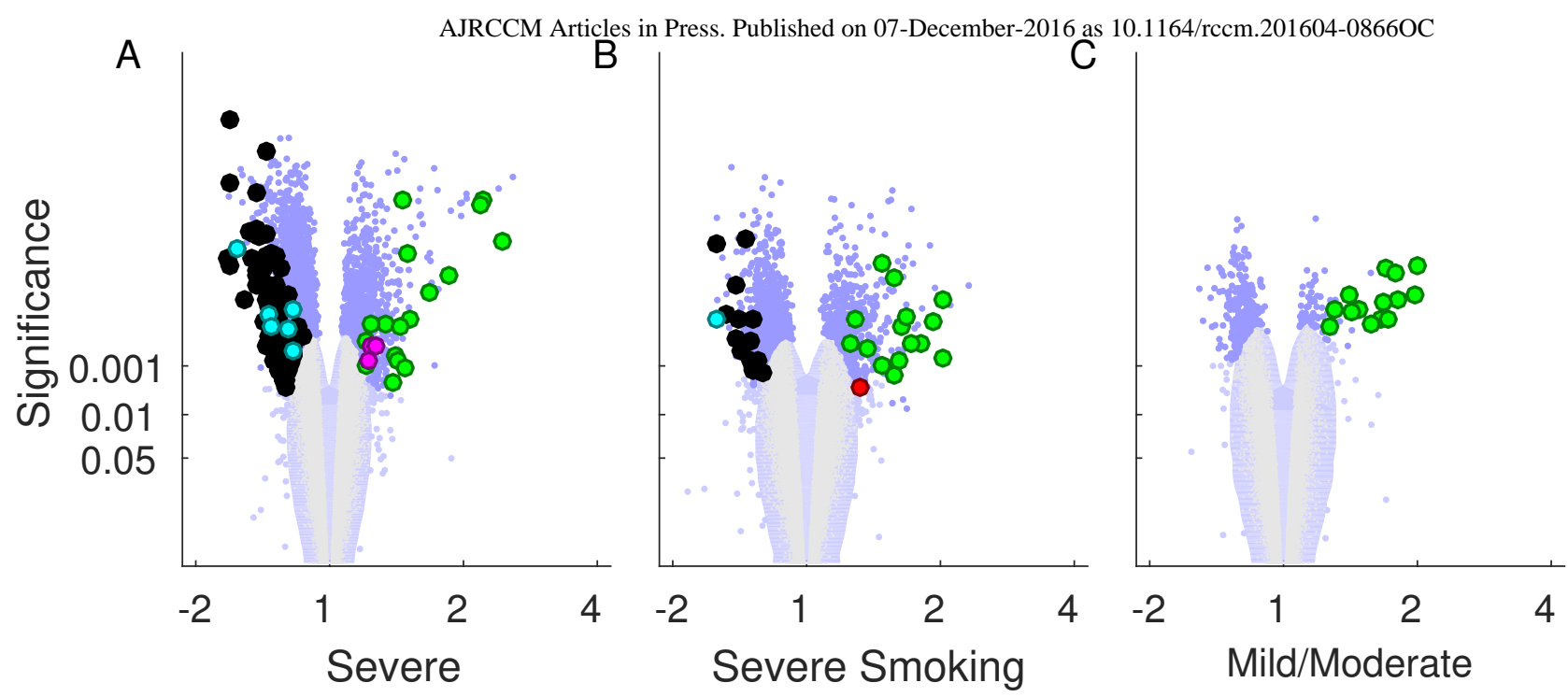

Figure 1

Fold Difference Relative to Non-Asthmatics 


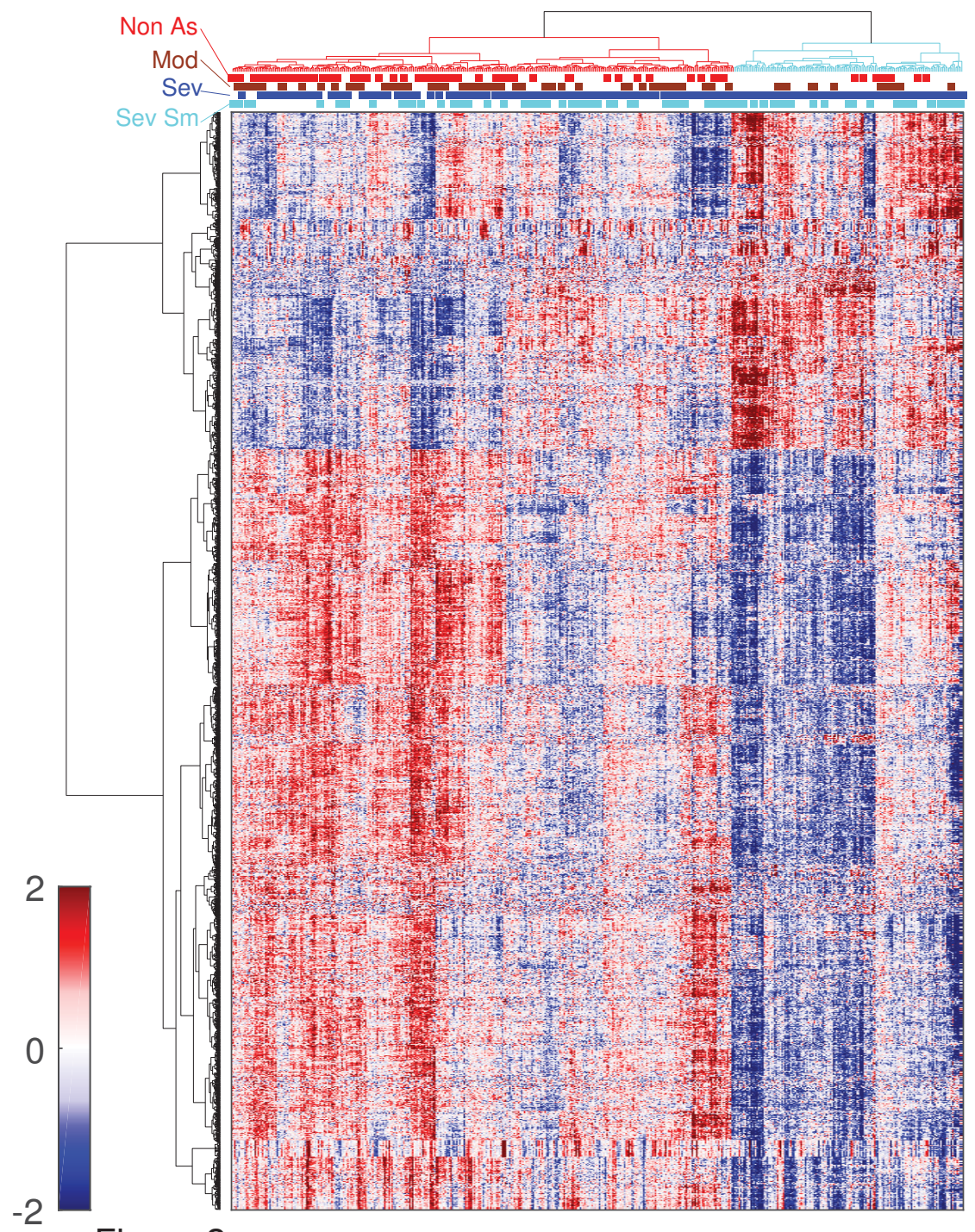

Figure 2a 


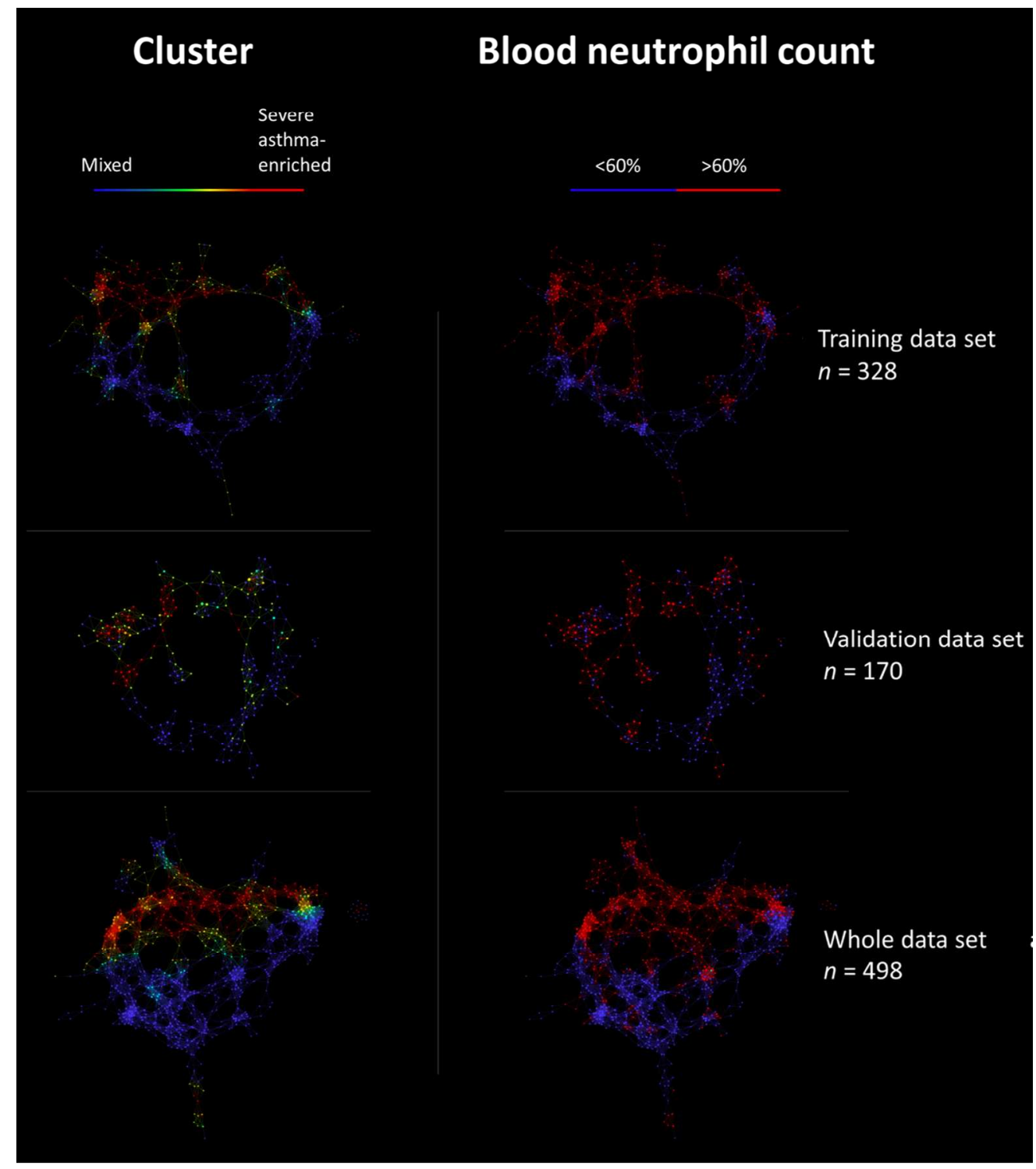

Figure $2 b$

$242 \times 275 \mathrm{~mm}(150 \times 150 \mathrm{DPI})$ 


\section{Online Data Supplement}

A Severe Asthma Disease Signature from Gene Expression Profiling of Peripheral Blood from UBIOPRED Cohorts

J. Bigler $^{1 *}$, M. Boedigheimer ${ }^{2}$, J.P.R. Schofield ${ }^{3}$, P.J. Skipp ${ }^{3}$, J. Corfield $^{4}$, A. Rowe $^{5}$, A.R. Sousa ${ }^{6}$, M.

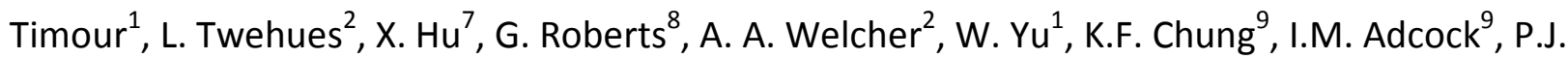
Sterk $^{10}$, and R. Djukanovic ${ }^{8}$ on behalf of The U-BIOPRED Study Group with input from the UBIOPRED Patient Input Platform and patient representatives from the Ethics Board and Safety Management Board. 


\section{Supplementary Methods}

\section{Categorization of the Study Population}

The participants were categorized into four groups:

Severe non-smoking asthma: Participants in this group were non-smokers with a less than five pack-year smoking history, with asthma and uncontrolled symptoms defined according to GINA guidelines and/or frequent exacerbations (more than two per year) despite high-dose inhaled corticosteroids (ICS) (ICS $\geq 1000 \mu \mathrm{g}$ fluticasone propionate/day or equivalent dose).

- $\quad$ Smokers and ex-smokers with severe asthma: This group was defined as for the severe asthma group except that they were either current smokers or ex-smokers with a pack history of at least five years.

- $\quad$ Mild/moderate non-smoking asthmatics: These non-smoking participants had controlled or partially controlled asthma symptoms, as defined by the Global Initiative for Asthma (GINA), whilst receiving a dose of less than $500 \mu \mathrm{g}$ fluticasone propionate/day or equivalent.

- $\quad$ Healthy non-smoking controls (HC): These participants had no history of asthma or wheeze, had no other chronic respiratory disease, had never smoked and their prebronchodilator $\mathrm{FEV}_{1}$ was $\geq 80 \%$ predicted.

\footnotetext{
Atopy

Atopy was diagnosed either by skin tests or RAST analysis of six common aero-allergens as described in more detail in the Supplement to Shaw et al. (1).
} 
Skin prick testing was performed to common allergens using single headed lancet and positive $(10 \mathrm{mg} / \mathrm{ml}$ histamine $)$ and negative (saline) controls. A positive skin prick test was defined as a wheal $\geq 3 \mathrm{~mm}$ to common aeroallergens (Dermatophagoides pteronyssinus, Dermatophagoides farinae, cat, dog, grass pollen mixture, tree pollen mixture and aspergillus plus the following were they were relevant to the site: cockroach and Olea europea and Parietaria) in the presence of positive $(\geq 3 \mathrm{~mm})$ and negative $(0 \mathrm{~mm})$ controls.

Total IgE and specific IgE tests to the six common aeroallergens were measured. Allergic sensitization was defined as at least one test with a specific IgE $\geq 0.34 I U$. These measurements were made in the laboratory of the individual site.

\section{Statistical Analysis}

Differentially expressed genes (DEGs) and gene signatures were identified using an ANOVA model including covariates for RIN, site and gender. Permutation testing was used to estimate a null distribution and an iso-density line encircled the area containing most null results (99.99\%). The area is referred to as the patch of disbelief (PoD). A local false discovery rate (IFDR) of $<0.05$ was estimated using methods similar to those previously described $(2,3)$. DEGs are those results outside the PoD with an IFDR $<0.05$.

In some analyses, white blood cell counts (WBC) were used as covariates to capture the total abundance of cells. Percentages of eosinophils, basophils, neutrophils, monocytes, and lymphocytes were used to model the proportion of that cell type in the blood. Zero values were 
imputed at $1 / 2$ the observed minimum of non-zero values. Data were log2 transformed to improve normality

Hierarchical Clustering was performed on data standardized to have a mean of zero and standard deviation of one. A Euclidean distance metric and Ward linkage were used. For validation purposes, clustering was done on various datasets (e.g. training and validation) in combination with various gene lists (e.g. severe asthma signature developed from the training set). Cutting the top branch formed two clusters. A cross-tabulation of number of participants by cohort and cluster were evaluated for enrichment using a chi-squared test. Characteristics that were tested included lung function (including $\mathrm{FEV}_{1}$, methacholine challenge, airflow limitation, forced oscillation technique, plethysmography, spirometry), exacerbation frequency, blood chemistry, lung biopsy and sputum differential cell count, medication use (inhaled corticosteroid and medications other than asthma medication), ACQ, AQLQ, ESS, HADS, and MARS questionnaires, demographics, family history, medical history, and allergies.

Functional analysis of DEG sets and predictors of upstream regulators were identified using Ingenuity Pathway Analysis (IPA; QIAGEN, Redwood City, CA). The pathway analysis examines how many known targets of each disease, function, or regulator are present in the submitted data set and also compares the direction of change. A p-value for the overlap of dataset and known pathways and an activation score for the likely activation state that takes into account the direction of differences were generated. 


\section{Gene Correlation Network Analysis}

Gene expression correlation matrices were created using subsets of genes as described in the text. The absolute value of the correlation matrices was scaled (each element raised to some power) to make them approximately scale free. Generalized topological overlay (GTOM) (4) was applied to the scaled correlation matrices. Graphs were created by adding all edges with a GTOM value greater than a variable threshold and monitoring the number of connected components formed at each threshold. A bounded search algorithm was used to identify a threshold that maximized number of connected components. Each connected component was one gene module.

\section{References:}

1. Shaw DE, Sousa AR, Fowler SJ, Fleming LJ, Roberts G, Corfield J, Pandis I, Bansal AT, Bel EH, Auffray C, Compton CH, Bisgaard H, Bucchioni E, Caruso M, Chanez P, Dahlén B, Dahlen S-E, Dyson K, Frey U, Geiser T, Gerhardsson de Verdier M, Gibeon D, Guo Y-K, Hashimoto S, Hedlin G, Jeyasingham E, Hekking P-PW, Higenbottam T, Horváth I, et al. Clinical and inflammatory characteristics of the European U-BIOPRED adult severe asthma cohort. Eur Respir J $2015 ; 46: 1308-21$.

2. Efron B, Tibshirani R, Storey JD, Tusher V. Empirical Bayes Analysis of a Microarray Experiment. J Am Stat Assoc 2001;96:1151-1160.

3. Ploner A, Calza S, Gusnanto A, Pawitan Y. Multidimensional local false discovery rate for microarray studies. Bioinformatics 2006;22:556-565.

4. Yip AM, Horvath S. Gene network interconnectedness and the generalized topological overlap measure. BMC Bioinformatics 2007;8:22. 


\section{Supplementary Figures}

Supplementary Figure 1

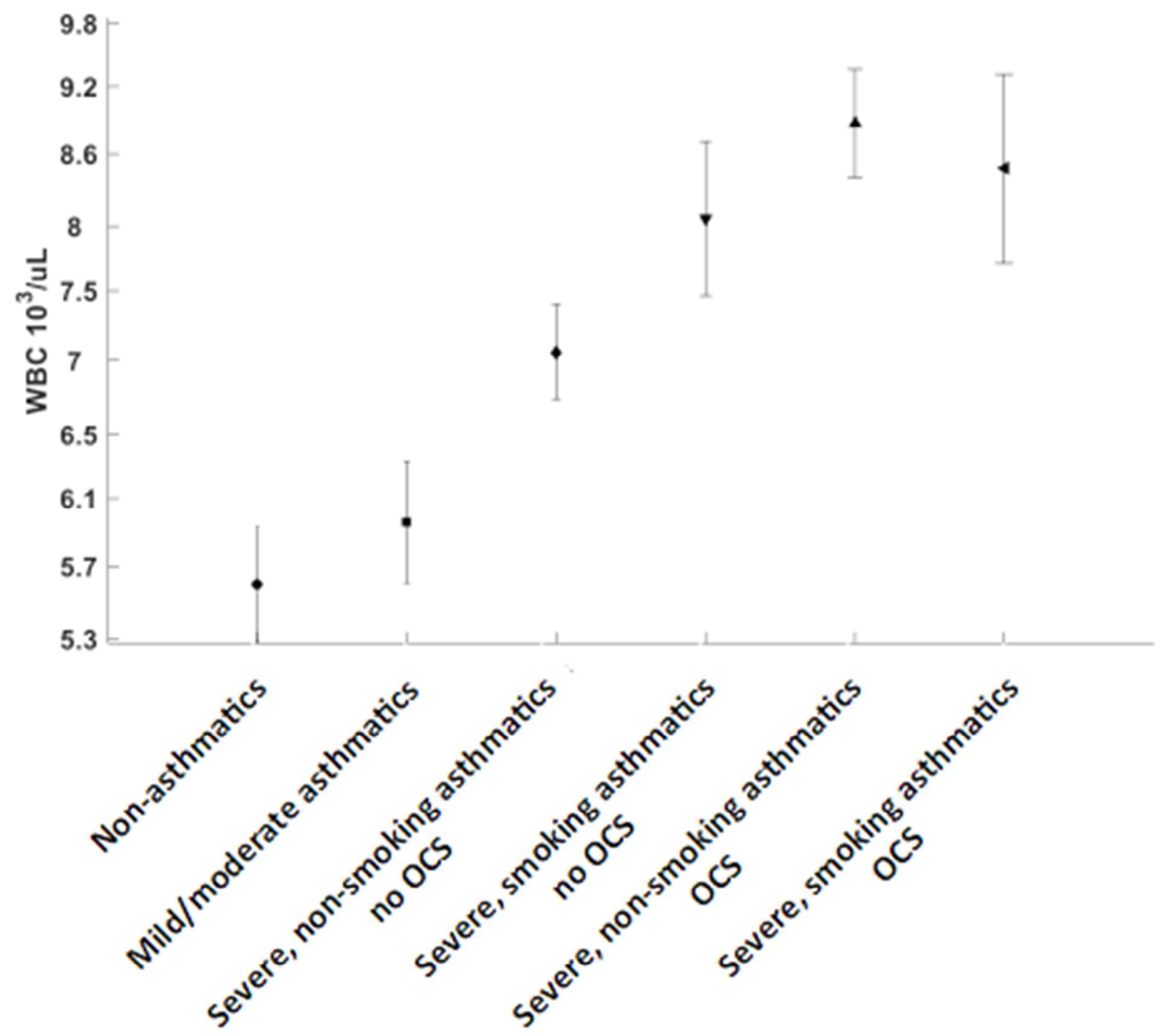

Mean white blood cell counts plotted for the non-asthmatics, mild/moderate asthmatics, and severe asthmatics. The latter group was divided by OCS use and smoking. Mean and 95\% confidence intervals are shown for each group. 


\section{Supplementary Figure 2}

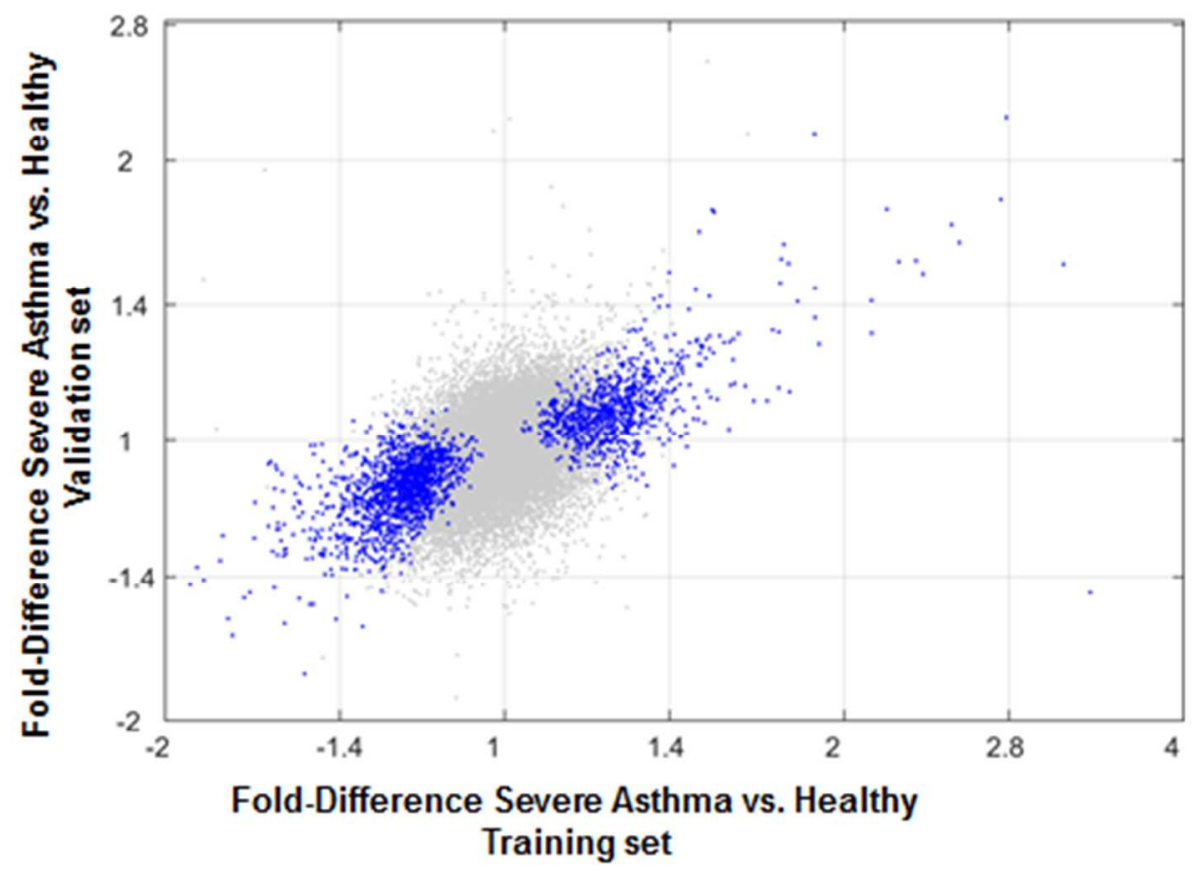

Comparison of gene expression differences between non-asthmatic and severe asthmatics were assessed separately in the training and the validation sets. The fold-change values for the two comparisons were then plotted against each other. Differences between the two data sets generally trended in the same direction, even though the magnitude of the effect may differ. Blue dots indicate reporters that were significantly different in the training set. 
Supplementary Figure 3

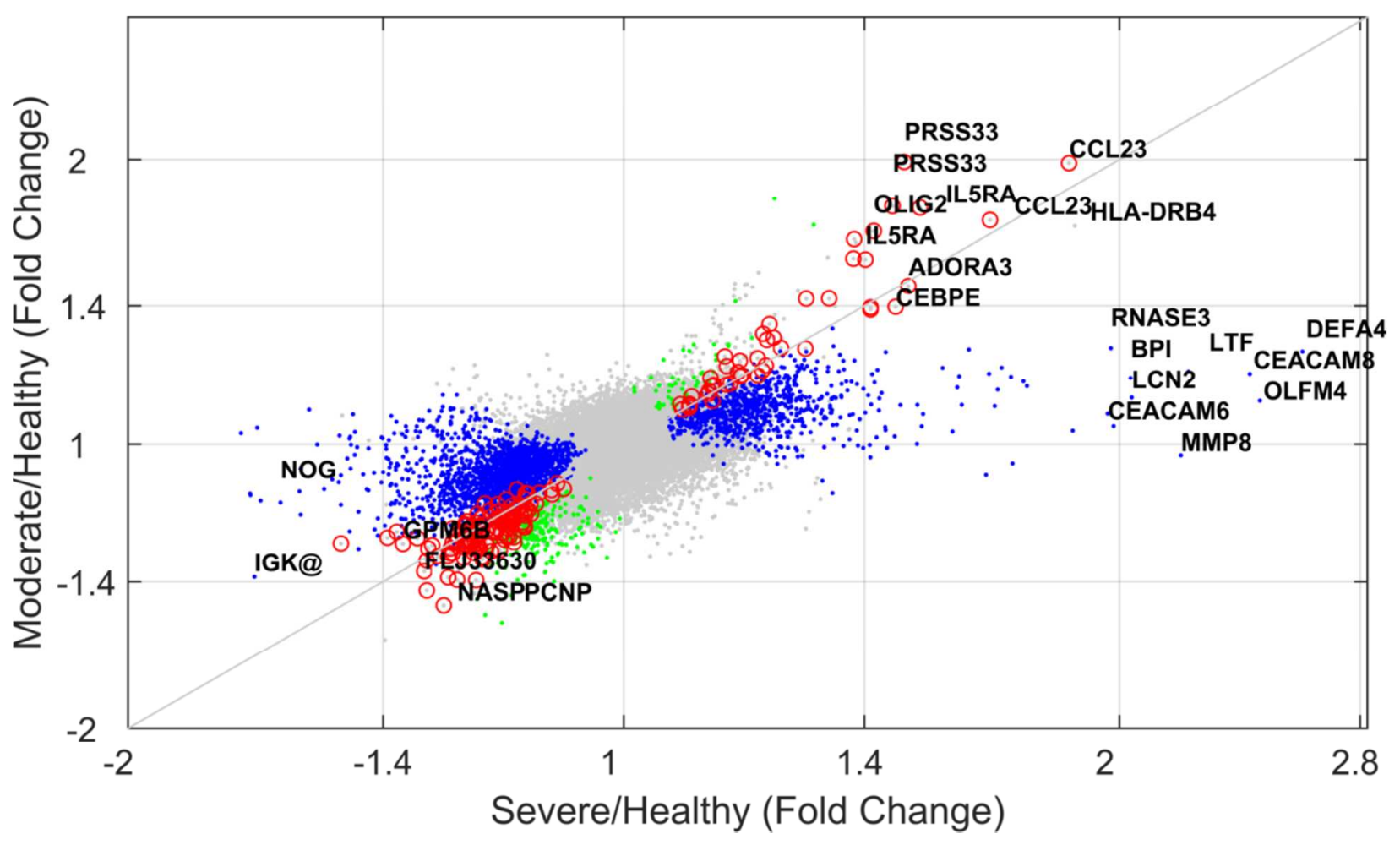

Comparison of gene expression differences between mild/moderate asthmatics or severe asthmatics and non-asthmatic individuals. The fold-change values for the two comparisons were plotted against each other. Perfect agreement between the two comparisons would put all the data points on the $45^{\circ}$ diagonal. The moderate and severe asthmatics shared differences to nonasthmatic individuals. The magnitude of the difference and the number of differentially expressed genes was larger in the severe asthmatic vs. non-asthmatic comparison. Blue, green and red indicate changes found in severe only, mild/moderate only or both cohorts compared to non-asthmatic volunteers. 


\section{Supplementary Figure 4}

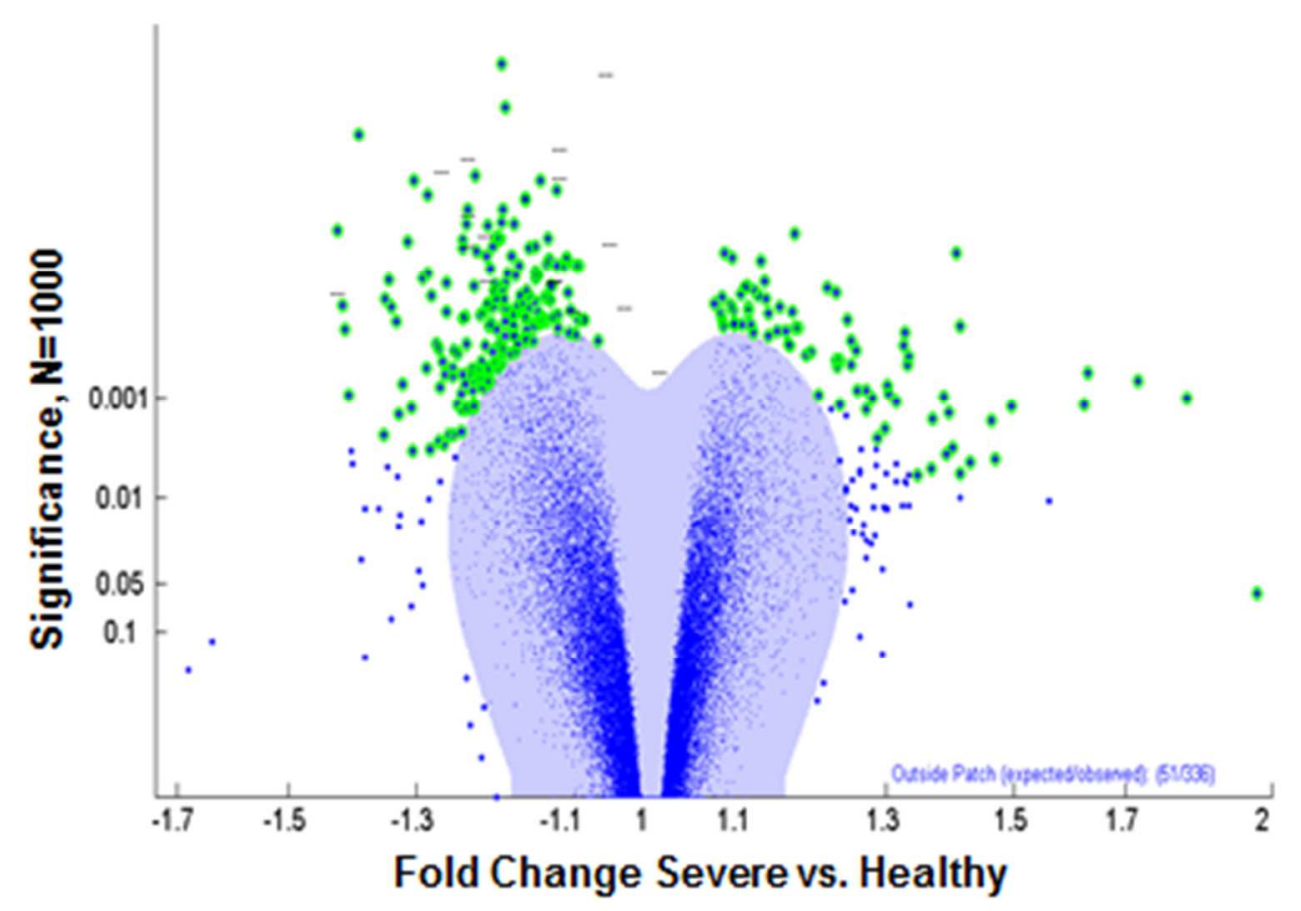

Comparison of non-smoking severe asthmatics with non-smoking non-asthmatic individuals. In this analysis, whole blood cell, basophil, eosinophil, neutrophil, lymphocyte, and monocyte counts were included as covariates. A positive fold-change indicates higher expression in asthmatics compared to non-asthmatic individuals and negative fold-change lower expression. Light blue shaded area: Patch of Disbelief (non-significant differences). Green data points represent reporters outside the Patch of Disbelief with a local false discovery rate of less than $5 \%$. 
Supplementary Figure 5

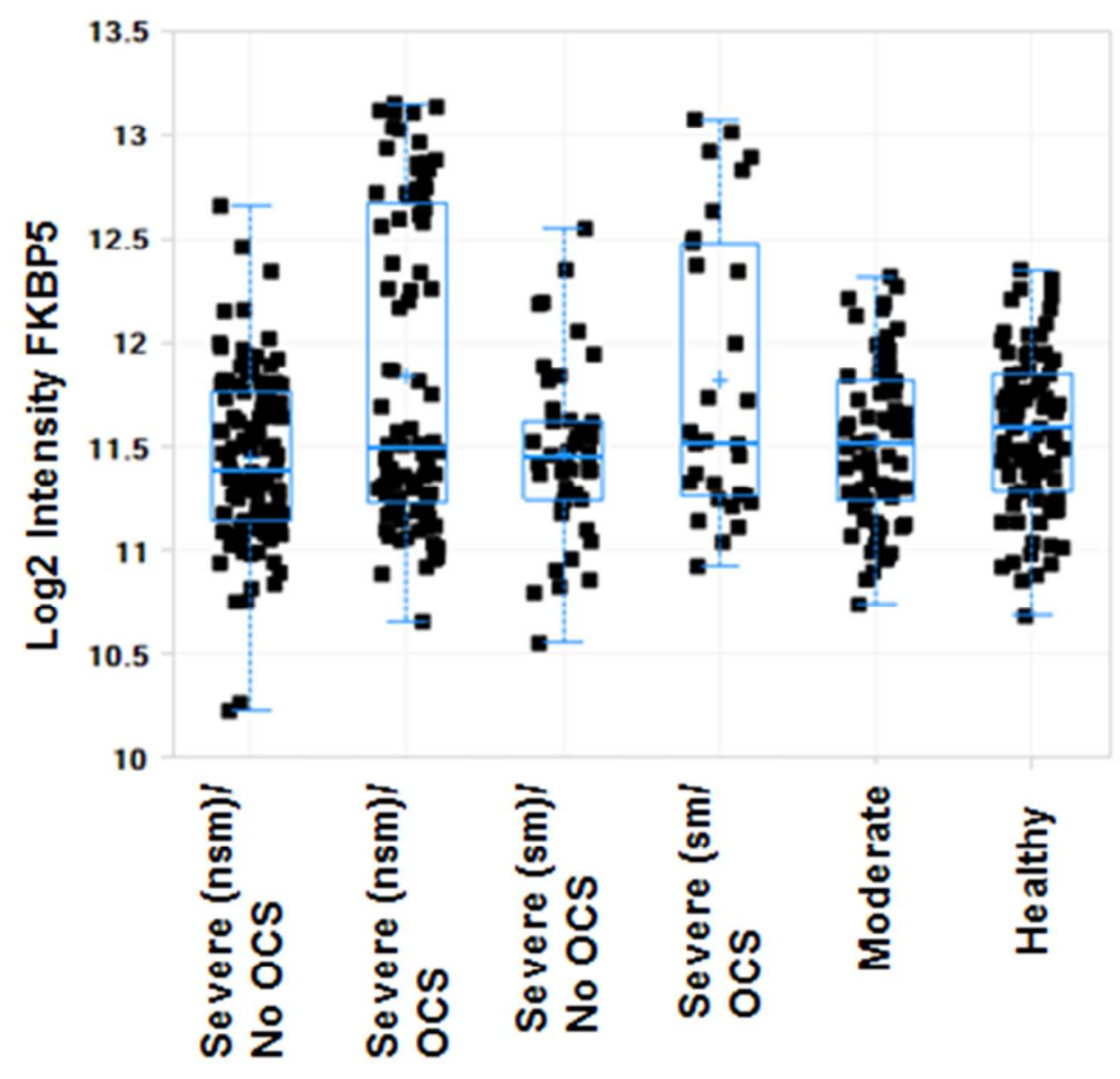

FKBP5 transcript levels by cohort and oral corticosteroid (OCS) use. The FKBP5 transcript levels were taken from microarrays. Elevated FKBP5 levels were detected only in cohorts reporting OCS use, but not all the patients taking OCS had elevated FKBP5 levels. 


\section{Supplementary Figure 6}

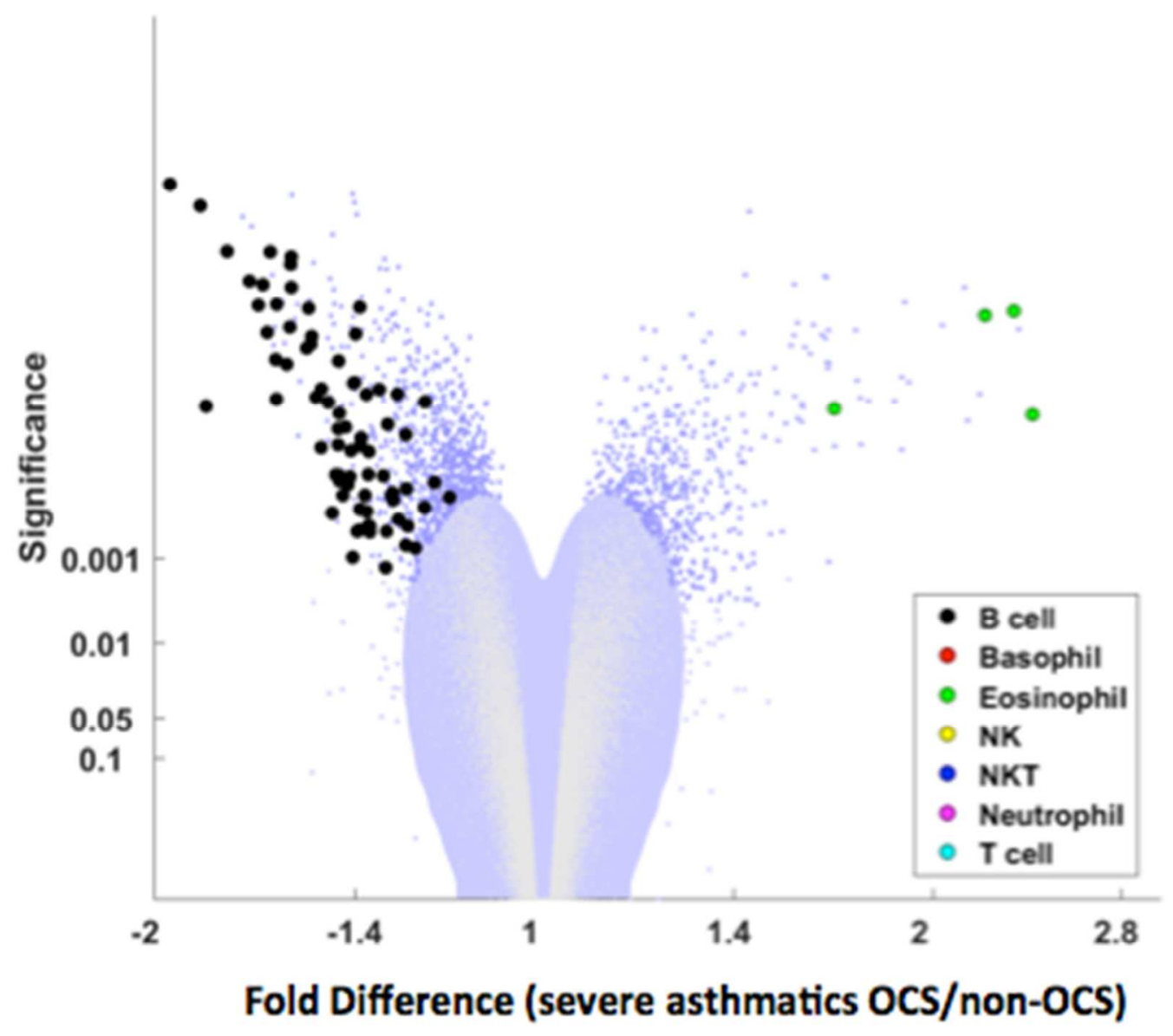

Comparison of non-smoking severe asthmatics on maintenance OCS treatment and non-

smoking severe asthmatics not on maintenance OCS. A positive fold-change indicates higher expression in OCS users when compared to non-users; negative fold-change indicates lower expression. Light blue shaded area: Patch of Disbelief (non-significant differences). Data points outside the Patch of Disbelief have a local false discovery rate $\leq 0.05$. Data points representing genes that are selective for a particular immune cell subtype are highlighted in different colors - for interpretation of color, see insert. 
Supplementary Figure 7

A
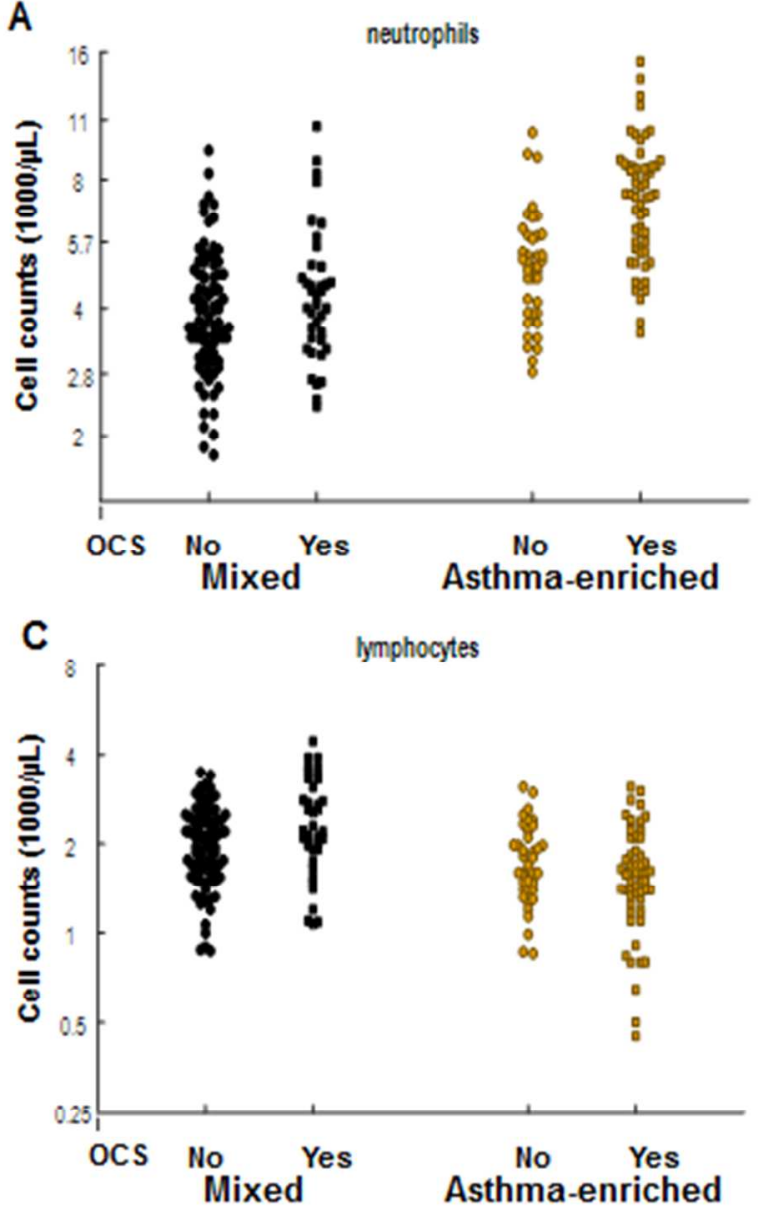

B

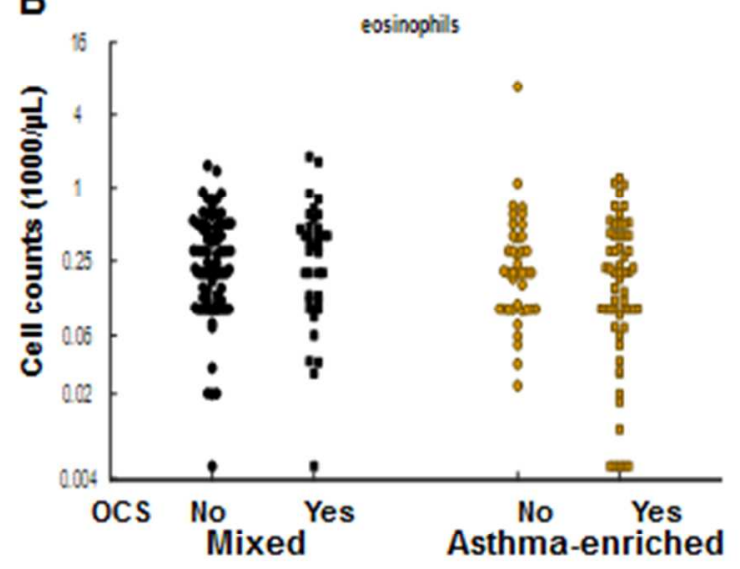

The cell counts for neutrophils (A), eosinophils (B), and lymphocytes (C) for the non-smoking severe asthmatics are shown by cluster as defined by transcriptional profiles and by oral corticosteroid use. 
Supplemental Table 1 - Sample Sets

\begin{tabular}{lccc}
\hline \multicolumn{1}{c}{ Description } & $\begin{array}{c}\text { Baseline } \\
\text { Training Set }\end{array}$ & $\begin{array}{c}\text { Baseline } \\
\text { Validation Set }\end{array}$ & $\begin{array}{c}\text { Total } \\
\text { Baseline }\end{array}$ \\
\hline Severe asthmatics/non-smoking & 165 & 81 & 246 \\
Severe asthmatics/smoking & 57 & 31 & 88 \\
Mild-moderate asthmatics/non-smoking & 50 & 27 & 77 \\
Non-asthmatic/non-smoking & 56 & 31 & 87 \\
\hline Total & $\mathbf{3 2 8}$ & $\mathbf{1 7 0}$ & $\mathbf{4 9 8}$ \\
\hline
\end{tabular}


Supplemental Table 2 - Cell-specific Genes

\begin{tabular}{|c|c|c|c|c|c|c|c|}
\hline symbol & probe_name & product & CellSpecific & foldChange & pval & IFDR & group \\
\hline TCF4 & 212382_PM_at & transcription factor 4 & B cell & -1.37 & 0.00 & 0.00 & severe asthma, smoking \\
\hline TSPAN13 & 217979_PM_at & tetraspanin 13 & B cell & -1.59 & 0.00 & 0.00 & severe asthma, smoking \\
\hline PAX5 & 221969_PM_at & paired box 5 & B cell & -1.44 & 0.00 & 0.00 & severe asthma, smoking \\
\hline \multirow[t]{2}{*}{ EBF1 } & 232204_PM_at & early B-cell factor 1 & B cell & -1.31 & 0.00 & 0.00 & severe asthma, smoking \\
\hline & 244394_PM_at & --- & B cell & -1.43 & 0.00 & 0.00 & severe asthma, smoking \\
\hline IGHM & 209374_PM_s_at & immunoglobulin heavy constant mu & B cell & -1.44 & 0.00 & 0.01 & severe asthma, smoking \\
\hline BLNK & 207655_PM_s_at & B-cell linker & B cell & -1.33 & 0.00 & 0.01 & severe asthma, smoking \\
\hline IGK@ & 237625_PM_s_at & immunoglobulin kappa locus & B cell & -1.52 & 0.00 & 0.01 & severe asthma, smoking \\
\hline EBF1 & 227646_PM_at & early B-cell factor 1 & B cell & -1.4 & 0.00 & 0.01 & severe asthma, smoking \\
\hline \multirow[t]{2}{*}{ MS4A1 } & 231418_PM_at & Membrane-spanning 4-domains, subfamily & B cell & -1.34 & 0.00 & 0.01 & severe asthma, smoking \\
\hline & 242104_PM_at & --- & B cell & -1.29 & 0.00 & 0.01 & severe asthma, smoking \\
\hline AFF3 & 227198_PM_at & AF4 & B cell & -1.33 & 0.00 & 0.03 & severe asthma, smoking \\
\hline EBF1 & 229487_PM_at & early B-cell factor 1 & B cell & -1.26 & 0.00 & 0.03 & severe asthma, smoking \\
\hline PLEKHG1 & 226122_PM_at & pleckstrin homology domain containing, far & B cell & -1.32 & 0.00 & 0.03 & severe asthma, smoking \\
\hline $\mathrm{HRH} 4$ & 221170_PM_at & histamine receptor $\mathrm{H} 4$ & Basophil & 1.32 & 0.00 & 0.05 & severe asthma, smoking \\
\hline LTF & 202018_PM_s_at & lactotransferrin & Eosinophil & 1.92 & 0.00 & 0.00 & severe asthma, smoking \\
\hline ADORA3 & 206171_PM_at & adenosine $\mathrm{A} 3$ receptor & Eosinophil & 1.57 & 0.00 & 0.00 & severe asthma, smoking \\
\hline OLFM4 & 212768_PM_s_at & olfactomedin 4 & Eosinophil & 2.04 & 0.00 & 0.00 & severe asthma, smoking \\
\hline CEBPE & 214523_PM_at & CCAAT & Eosinophil & 1.47 & 0.00 & 0.00 & severe asthma, smoking \\
\hline MMP8 & 231688_PM_at & matrix metallopeptidase 8 (neutrophil colla & Eosinophil & 2.03 & 0.00 & 0.00 & severe asthma, smoking \\
\hline PRSS33 & 1552348_PM_at & protease, serine, 33 & Eosinophil & 1.71 & 0.00 & 0.00 & severe asthma, smoking \\
\hline CCL23 & 210549_PM_s_at & chemokine ( $\mathrm{C}-\mathrm{C}$ motif) ligand 23 & Eosinophil & 1.81 & 0.00 & 0.00 & severe asthma, smoking \\
\hline ACPP & 204393_PM_s_at & acid phosphatase, prostate & Eosinophil & 1.29 & 0.00 & 0.00 & severe asthma, smoking \\
\hline IL5RA & 211517_PM_s_at & interleukin 5 receptor, alpha & Eosinophil & 1.64 & 0.00 & 0.00 & severe asthma, smoking \\
\hline OLIG2 & 213825_PM_at & oligodendrocyte lineage transcription facto & Eosinophil & 1.67 & 0.00 & 0.00 & severe asthma, smoking \\
\hline SMPD3 & 219695_PM_at & sphingomyelin phosphodiesterase 3 , neutra & Eosinophil & 1.37 & 0.00 & 0.01 & severe asthma, smoking \\
\hline SPNS3 & 235900_PM_at & spinster homolog 3 (Drosophila) & Eosinophil & 1.26 & 0.00 & 0.01 & severe asthma, smoking \\
\hline ALOX15 & 207328_PM_at & arachidonate 15-lipoxygenase & Eosinophil & 1.58 & 0.00 & 0.01 & severe asthma, smoking \\
\hline CCL23 & 210548_PM_at & chemokine (C-C motif) ligand 23 & Eosinophil & 1.61 & 0.00 & 0.01 & severe asthma, smoking \\
\hline IL5RA & 210744_PM_s_at & interleukin 5 receptor, alpha & Eosinophil & 1.48 & 0.00 & 0.03 & severe asthma, smoking \\
\hline NOG & 231798_PM_at & noggin & T cell & -1.59 & 0.00 & 0.01 & severe asthma, smoking \\
\hline$\overline{B L N K}$ & 207655_PM_s_at & B-cell linker & B cell & -1.46 & 0.00 & 0.00 & severe asthma \\
\hline
\end{tabular}




\begin{tabular}{|c|c|c|c|c|c|c|c|}
\hline IGHM & 209374_PM_s_at & immunoglobulin heavy constant mu & B cell & -1.49 & 0.00 & 0.00 & severe asthma \\
\hline TCL1A & 209995_PM_s_at & T-cell leukemia & B cell & -1.67 & 0.00 & 0.00 & severe asthma \\
\hline TCF4 & 212382_PM_at & transcription factor 4 & B cell & -1.39 & 0.00 & 0.00 & severe asthma \\
\hline IGHM & 212827_PM_at & immunoglobulin heavy constant mu & B cell & -1.46 & 0.00 & 0.00 & severe asthma \\
\hline TSPAN13 & 217979_PM_at & tetraspanin 13 & B cell & -1.69 & 0.00 & 0.00 & severe asthma \\
\hline PAX5 & 221969_PM_at & paired box 5 & B cell & -1.39 & 0.00 & 0.00 & severe asthma \\
\hline TCL1A & 39318_PM_at & T-cell leukemia & B cell & -1.71 & 0.00 & 0.00 & severe asthma \\
\hline AFF3 & 227198_PM_at & AF4 & B cell & -1.47 & 0.00 & 0.00 & severe asthma \\
\hline EBF1 & 227646_PM_at & early B-cell factor 1 & B cell & -1.52 & 0.00 & 0.00 & severe asthma \\
\hline MS4A1 & 231418_PM_at & Membrane-spanning 4-domains, subfamily & B cell & -1.45 & 0.00 & 0.00 & severe asthma \\
\hline FCRL1 & 235982_PM_at & Fc receptor-like 1 & B cell & -1.46 & 0.00 & 0.00 & severe asthma \\
\hline \multirow[t]{2}{*}{ IGK@ } & 237625_PM_s_at & immunoglobulin kappa locus & B cell & -1.68 & 0.00 & 0.00 & severe asthma \\
\hline & 242104_PM_at & --- & B cell & -1.38 & 0.00 & 0.00 & severe asthma \\
\hline \multirow[t]{2}{*}{ FCRL1 } & 243968_PM_x_at & Fc receptor-like 1 & B cell & -1.47 & 0.00 & 0.00 & severe asthma \\
\hline & 244394_PM_at & --- & B cell & -1.47 & 0.00 & 0.00 & severe asthma \\
\hline CD79A & 1555779̄PM _âatat & CD79a molecule, immunoglobulin-associat $\epsilon$ & B cell & -1.4 & 0.00 & 0.00 & severe asthma \\
\hline CCDC50 & 228693_PM_at & coiled-coil domain containing 50 & B cell & -1.35 & 0.00 & 0.00 & severe asthma \\
\hline EBF1 & 232204_PM_at & early B-cell factor 1 & B cell & -1.32 & 0.00 & 0.00 & severe asthma \\
\hline EBF1 & 229487_PM_at & early B-cell factor 1 & B cell & -1.34 & 0.00 & 0.00 & severe asthma \\
\hline MS4A1 & 217418_PM_x_at & membrane-spanning 4-domains, subfamily & B cell & -1.38 & 0.00 & 0.00 & severe asthma \\
\hline MS4A1 & 210356_PM_x_at & membrane-spanning 4-domains, subfamily & B cell & -1.37 & 0.00 & 0.00 & severe asthma \\
\hline \multirow[t]{2}{*}{ OSBPL10 } & 219073_PM_s_at & oxysterol binding protein-like 10 & B cell & -1.39 & 0.00 & 0.00 & severe asthma \\
\hline & 230648_PM_at & uncharacterized LOC283663 & B cell & -1.36 & 0.00 & 0.00 & severe asthma \\
\hline CD79A & 205049_PM_s_at & CD79a molecule, immunoglobulin-associat $\epsilon$ & B cell & -1.36 & 0.00 & 0.00 & severe asthma \\
\hline \multirow[t]{2}{*}{ COBLL1 } & 203642_PM_s_at & COBL-like 1 & B cell & -1.32 & 0.00 & 0.00 & severe asthma \\
\hline & 230245_PM_s_at & uncharacterized LOC283663 & B cell & -1.39 & 0.00 & 0.00 & severe asthma \\
\hline PLEKHG1 & 226122_PM_at & pleckstrin homology domain containing, far & B cell & -1.37 & 0.00 & 0.00 & severe asthma \\
\hline SPIB & 205861_PM_at & Spi-B transcription factor (Spi-1 & B cell & -1.28 & 0.00 & 0.00 & severe asthma \\
\hline IGHD & 230877_PM_at & immunoglobulin heavy constant delta & B cell & -1.55 & 0.00 & 0.00 & severe asthma \\
\hline CKAP2 & 217148_PM_x_at & Cytoskeleton associated protein 2 & B cell & -1.38 & 0.00 & 0.00 & severe asthma \\
\hline IGHD & 215621_PM_s_at & immunoglobulin heavy constant delta & B cell & -1.4 & 0.00 & 0.00 & severe asthma \\
\hline LARGE & 215543_PM_s_at & like-glycosyltransferase & B cell & -1.36 & 0.00 & 0.00 & severe asthma \\
\hline BLK & 206255_PM_at & B lymphoid tyrosine kinase & B cell & -1.24 & 0.00 & 0.00 & severe asthma \\
\hline BANK1 & 1558662_PM_s_at & B-cell scaffold protein with ankyrin repeats & B cell & -1.29 & 0.00 & 0.00 & severe asthma \\
\hline
\end{tabular}




\begin{tabular}{|c|c|c|c|c|c|c|c|}
\hline IGHA1 & 214916_PM_x_at & immunoglobulin heavy constant alpha 1 & B cell & -1.33 & 0.00 & 0.00 & severe asthma \\
\hline FCRLA & 235400_PM_at & Fc receptor-like $A$ & B cell & -1.3 & 0.00 & 0.00 & severe asthma \\
\hline FCRLA & 235372_PM_at & Fc receptor-like A & B cell & -1.32 & 0.00 & 0.00 & severe asthma \\
\hline CKAP2 & 216984_PM_x_at & Cytoskeleton associated protein 2 & B cell & -1.33 & 0.00 & 0.00 & severe asthma \\
\hline \multirow[t]{2}{*}{$\mathrm{CD} 22$} & 204581_PM_at & CD22 molecule & B cell & -1.3 & 0.00 & 0.00 & severe asthma \\
\hline & 243467_PM_at & --- & B cell & -1.18 & 0.00 & 0.00 & severe asthma \\
\hline KLHL14 & 228377_PM_at & kelch-like 14 (Drosophila) & B cell & -1.31 & 0.00 & 0.00 & severe asthma \\
\hline IGHV5-78 & 210450_PM_at & \multicolumn{2}{|c|}{ immunoglobulin heavy variable 5-78 (pseuc B cell } & -1.18 & 0.00 & 0.00 & severe asthma \\
\hline SNX22 & 235460_PM_at & sorting nexin 22 & B cell & -1.15 & 0.00 & 0.01 & severe asthma \\
\hline CKAP2 & 217235_PM_x_at & Cytoskeleton associated protein 2 & B cell & -1.34 & 0.00 & 0.01 & severe asthma \\
\hline MS4A1 & 228599_PM_at & membrane-spanning 4-domains, subfamily & B cell & -1.38 & 0.00 & 0.01 & severe asthma \\
\hline FCRL2 & 221239_PM_s_at & Fc receptor-like 2 & B cell & -1.21 & 0.00 & 0.01 & severe asthma \\
\hline MS4A1 & 228592_PM_at & membrane-spanning 4-domains, subfamily & B cell & -1.29 & 0.00 & 0.01 & severe asthma \\
\hline IGHG1 & 213674_PM_x_at & Immunoglobulin heavy constant gamma 1 ( & ( B cell & -1.38 & 0.00 & 0.01 & severe asthma \\
\hline SNX22 & 230509_PM_at & sorting nexin 22 & B cell & -1.24 & 0.00 & 0.01 & severe asthma \\
\hline BANK1 & 222915_PM_s_at & B-cell scaffold protein with ankyrin repeats & B cell & -1.21 & 0.00 & 0.01 & severe asthma \\
\hline BTLA & 236226_PM_at & B and T lymphocyte associated & B cell & -1.21 & 0.00 & 0.01 & severe asthma \\
\hline \multirow[t]{3}{*}{ IGK@ } & 214768_PM_x_at & immunoglobulin kappa locus & B cell & -1.31 & 0.00 & 0.01 & severe asthma \\
\hline & 217251_PM_x_at & --- & B cell & -1.24 & 0.00 & 0.01 & severe asthma \\
\hline & 239244_PM_at & uncharacterized LOC100507616 & B cell & -1.27 & 0.00 & 0.02 & severe asthma \\
\hline IGHG1 & 228518_PM_at & \multicolumn{2}{|c|}{ immunoglobulin heavy constant gamma 1 ( $B$ cell } & -1.23 & 0.00 & 0.02 & severe asthma \\
\hline FAM129C & 1553369_PM_at & \multicolumn{2}{|c|}{ family with sequence similarity 129, memb $B$ cell } & -1.23 & 0.00 & 0.02 & severe asthma \\
\hline \multirow[t]{2}{*}{ IGK@ } & 216576_PM_x_at & immunoglobulin kappa locus & B cell & -1.3 & 0.00 & 0.02 & severe asthma \\
\hline & 211645_PM_x_at & --- & B cell & -1.29 & 0.00 & 0.02 & severe asthma \\
\hline IGK@ & 211644_PM_x_at & immunoglobulin kappa locus & B cell & -1.29 & 0.00 & 0.02 & severe asthma \\
\hline CKAP2 & 216566_PM_at & Cytoskeleton associated protein 2 & B cell & -1.33 & 0.00 & 0.02 & severe asthma \\
\hline FCRL5 & 224404_PM_s_at & Fc receptor-like 5 & B cell & -1.29 & 0.00 & 0.03 & severe asthma \\
\hline CNTNAP2 & 219301_PM_s_at & contactin associated protein-like 2 & B cell & -1.25 & 0.00 & 0.03 & severe asthma \\
\hline IGKC & 216207_PM_x_at & immunoglobulin kappa constant & B cell & -1.27 & 0.00 & 0.03 & severe asthma \\
\hline IGKC & 215217_PM_at & Immunoglobulin kappa constant & B cell & -1.27 & 0.00 & 0.03 & severe asthma \\
\hline \multirow[t]{3}{*}{ IGLV1-44 } & 234707_PM_x_at & immunoglobulin lambda variable 1-44 & B cell & -1.26 & 0.00 & 0.04 & severe asthma \\
\hline & 217480_PM_x_at & ig kappa chain V-I region Walker-like & B cell & -1.25 & 0.00 & 0.04 & severe asthma \\
\hline & 217378_PM_x_at & ig kappa chain V-I region Walker-like & B cell & -1.25 & 0.00 & 0.05 & severe asthma \\
\hline LTF & 202018_PM_s_at & lactotransferrin & Eosinophil & 2.2 & 0.00 & 0.00 & severe asthma \\
\hline
\end{tabular}




\begin{tabular}{|c|c|c|c|c|c|c|c|}
\hline ADORA3 & 206171_PM_at & adenosine $\mathrm{A} 3$ receptor & Eosinophil & 1.49 & 0.00 & 0.00 & severe asthma \\
\hline CCL23 & 210548_PM_at & chemokine ( $\mathrm{C}-\mathrm{C}$ motif) ligand 23 & Eosinophil & 1.67 & 0.00 & 0.00 & severe asthma \\
\hline CCL23 & 210549_PM_s_at & chemokine ( $\mathrm{C}-\mathrm{C}$ motif) ligand 23 & Eosinophil & 1.86 & 0.00 & 0.00 & severe asthma \\
\hline OLFM4 & 212768_PM_s_at & olfactomedin 4 & Eosinophil & 2.43 & 0.00 & 0.00 & severe asthma \\
\hline CEBPE & 214523_PM_at & CCAAT & Eosinophil & 1.46 & 0.00 & 0.00 & severe asthma \\
\hline MMP8 & 231688_PM_at & matrix metallopeptidase 8 (neutrophil colla & Eosinophil & 2.18 & 0.00 & 0.00 & severe asthma \\
\hline SMPD3 & 219695_PM_at & sphingomyelin phosphodiesterase 3 , neutra & Eosinophil & 1.33 & 0.00 & 0.00 & severe asthma \\
\hline PIK3R6 & 1558770_PM_a_at & phosphoinositide-3-kinase, regulatory subu & Eosinophil & 1.23 & 0.00 & 0.00 & severe asthma \\
\hline ADORA3 & 223660_PM_at & adenosine $\mathrm{A} 3$ receptor & Eosinophil & 1.44 & 0.00 & 0.00 & severe asthma \\
\hline IL5RA & 211517_PM_s_at & interleukin 5 receptor, alpha & Eosinophil & 1.51 & 0.00 & 0.00 & severe asthma \\
\hline ACPP & 204393_PM_s_at & acid phosphatase, prostate & Eosinophil & 1.21 & 0.00 & 0.01 & severe asthma \\
\hline OLIG2 & 213825_PM_at & oligodendrocyte lineage transcription facto & Eosinophil & 1.42 & 0.00 & 0.01 & severe asthma \\
\hline IL5RA & 210744_PM_s_at & interleukin 5 receptor, alpha & Eosinophil & 1.4 & 0.00 & 0.01 & severe asthma \\
\hline PRSS33 & 1552348_PM_at & protease, serine, 33 & Eosinophil & 1.48 & 0.00 & 0.02 & severe asthma \\
\hline GPR97 & 220404_PM_at & G protein-coupled receptor 97 & Eosinophil & 1.21 & 0.00 & 0.03 & severe asthma \\
\hline SIGLEC8 & 208253_PM_at & sialic acid binding Ig-like lectin 8 & Eosinophil & 1.38 & 0.00 & 0.04 & severe asthma \\
\hline SLC22A1 & 207201_PM_s_at & solute carrier family 22 (organic cation tran & Neutrophil & 1.23 & 0.00 & 0.01 & severe asthma \\
\hline KCNJ15 & 238428_PM_at & potassium inwardly-rectifying channel, subf & f Neutrophil & 1.26 & 0.00 & 0.01 & severe asthma \\
\hline ALPL & 215783_PM_s_at & alkaline phosphatase, liver & Neutrophil & 1.22 & 0.00 & 0.02 & severe asthma \\
\hline NOG & 231798_PM_at & noggin & T cell & -1.62 & 0.00 & 0.00 & severe asthma \\
\hline LEF1-AS1 & 243362_PM_s_at & LEF1 antisense RNA 1 (non-protein coding) & T cell & -1.37 & 0.00 & 0.00 & severe asthma \\
\hline GAL3ST4 & 219815_PM_at & galactose-3-O-sulfotransferase 4 & T cell & -1.21 & 0.00 & 0.00 & severe asthma \\
\hline LEF1-AS1 & 243363_PM_at & LEF1 antisense RNA 1 (non-protein coding) & T cell & -1.36 & 0.00 & 0.00 & severe asthma \\
\hline YME1L1 & 234849_PM_at & YME1-like 1 (S. cerevisiae) & T cell & -1.23 & 0.00 & 0.00 & severe asthma \\
\hline DPP4 & 203717_PM_at & dipeptidyl-peptidase 4 & T cell & -1.2 & 0.00 & 0.01 & severe asthma \\
\hline$\overline{A L O X 15}$ & 207328_PM_at & arachidonate 15-lipoxygenase & Eosinophil & 1.82 & 0.00 & 0.00 & moderate asthma \\
\hline IDO1 & 210029_PM_at & indoleamine 2,3-dioxygenase 1 & Eosinophil & 1.71 & 0.00 & 0.00 & moderate asthma \\
\hline CCL23 & 210549_PM_s_at & chemokine ( $\mathrm{C}-\mathrm{C}$ motif) ligand 23 & Eosinophil & 1.98 & 0.00 & 0.00 & moderate asthma \\
\hline IL5RA & 211517_PM_s_at & interleukin 5 receptor, alpha & Eosinophil & 1.78 & 0.00 & 0.00 & moderate asthma \\
\hline OLIG2 & 213825_PM_at & oligodendrocyte lineage transcription facto & Eosinophil & 1.68 & 0.00 & 0.00 & moderate asthma \\
\hline PRSS33 & 1552348_PM_at & protease, serine, 33 & Eosinophil & 1.99 & 0.00 & 0.00 & moderate asthma \\
\hline CCL23 & 210548_PM_at & chemokine ( $\mathrm{C}-\mathrm{C}$ motif) ligand 23 & Eosinophil & 1.73 & 0.00 & 0.00 & moderate asthma \\
\hline SIGLEC8 & 208253_PM_at & sialic acid binding Ig-like lectin 8 & Eosinophil & 1.65 & 0.00 & 0.00 & moderate asthma \\
\hline CEBPE & 214523_PM_at & CCAAT & Eosinophil & 1.40 & 0.00 & 0.01 & moderate asthma \\
\hline
\end{tabular}




\begin{tabular}{lllrlrl} 
IL5RA & 210744_PM_S_at & interleukin 5 receptor, alpha & Eosinophil & 1.57 & 0.00 & 0.01 moderate asthma \\
ADORA3 & 206171_PM_at & adenosine A3 receptor & Eosinophil & 1.47 & 0.00 & 0.01 moderate asthma \\
PIK3R6 & 1558770_PM_a_at & phosphoinositide-3-kinase, regulatory subu Eosinophil & 1.30 & 0.00 & 0.01 moderate asthma \\
SMPD3 & 219695_PM_at & sphingomyelin phosphodiesterase 3, neutrá Eosinophil & 1.43 & 0.00 & 0.02 moderate asthma \\
SPNS3 & 235900_PM_at & spinster homolog 3 (Drosophila) & Eosinophil & 1.27 & 0.00 & 0.03 moderate asthma \\
\hline
\end{tabular}


Supplemental Table 3 - Severe Asthma Disease Signature

\begin{tabular}{|c|c|c|c|c|c|}
\hline symbol & probe_name & product & foldChange & pval & IFDR \\
\hline TCL1A & 39318_PM_at & T-cell leukemia & -1.71 & 0.00 & 0.00 \\
\hline TSPAN13 & 217979_PM_at & tetraspanin 13 & -1.69 & 0.00 & 0.00 \\
\hline EBF1 & 233261_PM_at & Early B-cell factor 1 & -1.69 & 0.00 & 0.00 \\
\hline IGK@ & 237625_PM_s_at & immunoglobulin kappa locus & -1.68 & 0.00 & 0.00 \\
\hline TCL1A & 209995_PM_s_at & T-cell leukemia & -1.67 & 0.00 & 0.00 \\
\hline LRRN3 & 209841_PM_s_at & leucine rich repeat neuronal 3 & -1.66 & 0.00 & 0.00 \\
\hline NOG & 231798_PM_at & noggin & -1.62 & 0.00 & 0.00 \\
\hline--- & 243780_PM_at & --- & -1.59 & 0.00 & 0.00 \\
\hline--- & 236307_PM_at & --- & -1.57 & 0.00 & 0.00 \\
\hline--- & 244696_PM_at & --- & -1.57 & 0.00 & 0.00 \\
\hline LRRN3 & 209840_PM_s_at & leucine rich repeat neuronal 3 & -1.57 & 0.00 & 0.00 \\
\hline IGHD & 230877_PM_at & immunoglobulin heavy constant delta & -1.55 & 0.00 & 0.00 \\
\hline--- & 232286_PM_at & --- & -1.54 & 0.00 & 0.00 \\
\hline EBF1 & 227646_PM_at & early B-cell factor 1 & -1.52 & 0.00 & 0.00 \\
\hline--- & 244172_PM_at & --- & -1.5 & 0.00 & 0.00 \\
\hline CUX2 & 213920_PM_at & cut-like homeobox 2 & -1.49 & 0.00 & 0.00 \\
\hline--- & 244876_PM_at & --- & -1.49 & 0.00 & 0.00 \\
\hline--- & 1557580_PM_at & --- & -1.49 & 0.00 & 0.00 \\
\hline IGHM & 209374_PM_s_at & immunoglobulin heavy constant mu & -1.49 & 0.00 & 0.00 \\
\hline--- & 233401_PM_at & --- & -1.48 & 0.00 & 0.00 \\
\hline AFF3 & 227198_PM_at & AF4 & -1.47 & 0.00 & 0.00 \\
\hline--- & 244394_PM_at & --- & -1.47 & 0.00 & 0.00 \\
\hline--- & 1557581_PM_x_at & --- & -1.47 & 0.00 & 0.00 \\
\hline FCRL1 & 243968_PM_x_at & Fc receptor-like 1 & -1.47 & 0.00 & 0.00 \\
\hline BLNK & 207655_PM_s_at & B-cell linker & -1.46 & 0.00 & 0.00 \\
\hline--- & 241577_PM_at & --- & -1.46 & 0.00 & 0.00 \\
\hline--- & 1566880_PM_at & --- & -1.46 & 0.00 & 0.00 \\
\hline FCRL1 & 235982_PM_at & Fc receptor-like 1 & -1.46 & 0.00 & 0.00 \\
\hline IGHM & 212827_PM_at & immunoglobulin heavy constant mu & -1.46 & 0.00 & 0.00 \\
\hline--- & 224348_PM_s_at & --- & -1.45 & 0.00 & 0.00 \\
\hline MS4A1 & 231418_PM_at & Membrane-spanning 4-domains, subfamily & -1.45 & 0.00 & 0.00 \\
\hline
\end{tabular}




\begin{tabular}{|c|c|c|c|c|c|}
\hline--- & 240861_PM_at & --- & -1.45 & 0.00 & 0.00 \\
\hline AKAP2 & 202760_PM_s_at & A kinase (PRKA) anchor protein 2 & -1.44 & 0.00 & 0.00 \\
\hline STRBP & 233252_PM_s_at & spermatid perinuclear RNA binding protein & -1.43 & 0.00 & 0.00 \\
\hline--- & 1556451_PM_at & --- & -1.43 & 0.00 & 0.00 \\
\hline IFI44L & 204439_PM_at & interferon-induced protein 44-like & -1.43 & 0.00 & 0.03 \\
\hline IL6ST & 234474_PM_x_at & interleukin 6 signal transducer (gp130, oncc & -1.42 & 0.00 & 0.00 \\
\hline--- & 236796_PM_at & --- & -1.42 & 0.00 & 0.00 \\
\hline--- & 241339_PM_at & --- & -1.41 & 0.00 & 0.00 \\
\hline COL4A3 & 222073_PM_at & collagen, type IV, alpha 3 (Goodpasture ant & -1.41 & 0.00 & 0.00 \\
\hline MAN1C1 & 214180_PM_at & mannosidase, alpha, class $1 C$, member 1 & -1.4 & 0.00 & 0.00 \\
\hline LOC100506195 & 1563039_PM_at & uncharacterized LOC100506195 & -1.4 & 0.00 & 0.00 \\
\hline $\mathrm{ABCB} 4$ & 1570505_PM_at & ATP-binding cassette, sub-family B (MDR & -1.4 & 0.00 & 0.00 \\
\hline CD79A & 1555779_PM_a_at & CD79a molecule, immunoglobulin-associat $€$ & -1.4 & 0.00 & 0.00 \\
\hline IGHD & 215621_PM_s_at & immunoglobulin heavy constant delta & -1.4 & 0.00 & 0.01 \\
\hline TCF4 & 212382_PM_at & transcription factor 4 & -1.39 & 0.00 & 0.00 \\
\hline FAM129C & 230983_PM_at & family with sequence similarity 129, membe & -1.39 & 0.00 & 0.00 \\
\hline--- & 240593_PM_x_at & --- & -1.39 & 0.00 & 0.00 \\
\hline CR2 & 205544_PM_s_at & complement component $(3 d$ & -1.39 & 0.00 & 0.00 \\
\hline PAX5 & 221969_PM_at & paired box 5 & -1.39 & 0.00 & 0.00 \\
\hline HLA-DOB & 205671_PM_s_at & major histocompatibility complex, class II, [ & -1.39 & 0.00 & 0.00 \\
\hline--- & 1560971_PM_a_at & --- & -1.39 & 0.00 & 0.00 \\
\hline OSBPL10 & 219073_PM_s_at & oxysterol binding protein-like 10 & -1.39 & 0.00 & 0.00 \\
\hline LOC283663 & 230245_PM_s_at & uncharacterized LOC283663 & -1.39 & 0.00 & 0.00 \\
\hline COL5A3 & 218975_PM_at & collagen, type V, alpha 3 & -1.39 & 0.00 & 0.01 \\
\hline--- & 237334_PM_at & --- & -1.38 & 0.00 & 0.00 \\
\hline TTN & 241791_PM_at & titin & -1.38 & 0.00 & 0.00 \\
\hline--- & 1554963_PM_at & --- & -1.38 & 0.00 & 0.00 \\
\hline POU2AF1 & 205267_PM_at & POU class 2 associating factor 1 & -1.38 & 0.00 & 0.00 \\
\hline--- & 242104_PM_at & --- & -1.38 & 0.00 & 0.00 \\
\hline MS4A1 & 217418_PM_x_at & membrane-spanning 4-domains, subfamily & -1.38 & 0.00 & 0.00 \\
\hline EPPK1 & 232165_PM_at & epiplakin 1 & -1.38 & 0.00 & 0.00 \\
\hline CKAP2 & 217148_PM_x_at & Cytoskeleton associated protein 2 & -1.38 & 0.00 & 0.00 \\
\hline--- & 228297_PM_at & --- & -1.38 & 0.00 & 0.00 \\
\hline
\end{tabular}




\begin{tabular}{|c|c|c|c|c|c|}
\hline MS4A1 & 228599_PM_at & membrane-spanning 4-domains, subfamily & -1.38 & 0.00 & 0.01 \\
\hline IGHG1 & 213674_PM_x_at & Immunoglobulin heavy constant gamma 1 ( & -1.38 & 0.00 & 0.01 \\
\hline CCR6 & 206983_PM_at & chemokine ( $\mathrm{C}-\mathrm{C}$ motif) receptor 6 & -1.37 & 0.00 & 0.00 \\
\hline RAB11FIP3 & 228613_PM_at & RAB11 family interacting protein 3 (class II) & -1.37 & 0.00 & 0.00 \\
\hline AGMAT & 221648_PM_s_at & agmatine ureohydrolase (agmatinase) & -1.37 & 0.00 & 0.00 \\
\hline--- & 1558409_PM_at & --- & -1.37 & 0.00 & 0.00 \\
\hline MS4A1 & 210356_PM_x_at & membrane-spanning 4-domains, subfamily & -1.37 & 0.00 & 0.00 \\
\hline PLEKHG1 & 226122_PM_at & pleckstrin homology domain containing, far & -1.37 & 0.00 & 0.00 \\
\hline $\mathrm{CD} 22$ & 217422_PM_s_at & CD22 molecule & -1.37 & 0.00 & 0.00 \\
\hline FCRLA & 235401_PM_s_at & Fc receptor-like A & -1.37 & 0.00 & 0.00 \\
\hline LEF1-AS1 & 243362_PM_s_at & LEF1 antisense RNA 1 (non-protein coding) & -1.37 & 0.00 & 0.00 \\
\hline GATM & 203178_PM_at & glycine amidinotransferase (L-arginine:glyci & -1.37 & 0.00 & 0.00 \\
\hline GPM6B & 209167_PM_at & glycoprotein M6B & -1.36 & 0.00 & 0.00 \\
\hline--- & 242052_PM_at & --- & -1.36 & 0.00 & 0.00 \\
\hline BCL11A & 1559078_PM_at & B-cell CLL & -1.36 & 0.00 & 0.00 \\
\hline TNFRSF21 & 218856_PM_at & tumor necrosis factor receptor superfamily, & -1.36 & 0.00 & 0.00 \\
\hline IL6ST & 234967_PM_at & interleukin 6 signal transducer (gp130, oncc & -1.36 & 0.00 & 0.00 \\
\hline CD79A & 205049_PM_s_at & CD79a molecule, immunoglobulin-associat $\epsilon$ & -1.36 & 0.00 & 0.00 \\
\hline LOC283663 & 230648_PM_at & uncharacterized LOC283663 & -1.36 & 0.00 & 0.00 \\
\hline--- & 243107_PM_at & --- & -1.36 & 0.00 & 0.00 \\
\hline LARGE & 215543_PM_s_at & like-glycosyltransferase & -1.36 & 0.00 & 0.00 \\
\hline--- & 234838_PM_at & --- & -1.36 & 0.00 & 0.00 \\
\hline LOC389834 & 244631_PM_at & ankyrin repeat domain 57 pseudogene & -1.36 & 0.00 & 0.00 \\
\hline IGLC1 & 217138_PM_x_at & Immunoglobulin lambda constant 1 (Mcg m & -1.36 & 0.00 & 0.00 \\
\hline LEF1-AS1 & 243363_PM_at & LEF1 antisense RNA 1 (non-protein coding) & -1.36 & 0.00 & 0.01 \\
\hline COL5A3 & 52255_PM_s_at & collagen, type $\mathrm{V}$, alpha 3 & -1.36 & 0.00 & 0.02 \\
\hline SLC15A2 & 240159_PM_at & solute carrier family $15(\mathrm{H}+$ & -1.36 & 0.00 & 0.02 \\
\hline BCL11A & 222891_PM_s_at & B-cell CLL & -1.35 & 0.00 & 0.00 \\
\hline RAB30 & 228003_PM_at & RAB30, member RAS oncogene family & -1.35 & 0.00 & 0.00 \\
\hline--- & 1554964_PM_x_at & --- & -1.35 & 0.00 & 0.00 \\
\hline--- & 237916_PM_at & --- & -1.35 & 0.00 & 0.00 \\
\hline POU6F1 & 229809_PM_at & POU class 6 homeobox 1 & -1.35 & 0.00 & 0.00 \\
\hline PMEPA1 & 222450_PM_at & prostate transmembrane protein, androger & -1.35 & 0.00 & 0.00 \\
\hline
\end{tabular}




\begin{tabular}{|c|c|c|c|c|c|}
\hline CCDC50 & 228693_PM_at & coiled-coil domain containing 50 & -1.35 & 0.00 & 0.00 \\
\hline STAP1 & 1554343_PM_a_at & signal transducing adaptor family member: & -1.35 & 0.00 & 0.00 \\
\hline MZB1 & 223565_PM_at & marginal zone B and B1 cell-specific protein & -1.35 & 0.00 & 0.00 \\
\hline--- & 238309_PM_x_at & --- & -1.35 & 0.00 & 0.02 \\
\hline--- & 232796_PM_at & --- & -1.34 & 0.00 & 0.00 \\
\hline PDE7A & 1552343_PM_s_at & phosphodiesterase 7A & -1.34 & 0.00 & 0.00 \\
\hline--- & 239449_PM_at & --- & -1.34 & 0.00 & 0.00 \\
\hline $\mathrm{BACH} 2$ & 227173_PM_s_at & BTB and CNC homology 1 , basic leucine zipr & -1.34 & 0.00 & 0.00 \\
\hline EBF1 & 229487_PM_at & early B-cell factor 1 & -1.34 & 0.00 & 0.00 \\
\hline--- & 1561035_PM_at & --- & -1.34 & 0.00 & 0.00 \\
\hline EPPK1 & 232164_PM_s_at & epiplakin 1 & -1.34 & 0.00 & 0.00 \\
\hline CKAP2 & 216708_PM_x_at & Cytoskeleton associated protein 2 & -1.34 & 0.00 & 0.00 \\
\hline CKAP2 & 217235_PM_x_at & Cytoskeleton associated protein 2 & -1.34 & 0.00 & 0.01 \\
\hline LAMP3 & 205569_PM_at & Iysosomal-associated membrane protein 3 & -1.34 & 0.00 & 0.02 \\
\hline TCF4 & 212386_PM_at & transcription factor 4 & -1.33 & 0.00 & 0.00 \\
\hline TCF4 & 212387_PM_at & transcription factor 4 & -1.33 & 0.00 & 0.00 \\
\hline IL28RA & 244261_PM_at & interleukin 28 receptor, alpha (interferon, li & -1.33 & 0.00 & 0.00 \\
\hline--- & 1560259_PM_at & --- & -1.33 & 0.00 & 0.00 \\
\hline SPIB & 232739_PM_at & Spi-B transcription factor (Spi-1 & -1.33 & 0.00 & 0.00 \\
\hline LEF1 & 210948_PM_s_at & lymphoid enhancer-binding factor 1 & -1.33 & 0.00 & 0.00 \\
\hline--- & 1563217_PM_at & --- & -1.33 & 0.00 & 0.00 \\
\hline IGHA1 & 214916_PM_x_at & immunoglobulin heavy constant alpha 1 & -1.33 & 0.00 & 0.00 \\
\hline CKAP2 & 216984_PM_x_at & Cytoskeleton associated protein 2 & -1.33 & 0.00 & 0.00 \\
\hline SLC16A10 & 219915_PM_s_at & solute carrier family 16 , member 10 (aroma & -1.33 & 0.00 & 0.01 \\
\hline PRKXP1 & 235987_PM_at & protein kinase, $\mathrm{X}$-linked, pseudogene 1 & -1.33 & 0.00 & 0.01 \\
\hline CKAP2 & 216566_PM_at & Cytoskeleton associated protein 2 & -1.33 & 0.00 & 0.02 \\
\hline NRCAM & 204105_PM_s_at & neuronal cell adhesion molecule & -1.32 & 0.00 & 0.00 \\
\hline--- & 239411_PM_at & --- & -1.32 & 0.00 & 0.00 \\
\hline EBF1 & 232204_PM_at & early B-cell factor 1 & -1.32 & 0.00 & 0.00 \\
\hline--- & 236315_PM_at & --- & -1.32 & 0.00 & 0.00 \\
\hline--- & 242839_PM_at & --- & -1.32 & 0.00 & 0.00 \\
\hline FLJ33630 & 228686_PM_at & uncharacterized LOC644873 & -1.32 & 0.00 & 0.00 \\
\hline--- & 244610_PM_x_at & --- & -1.32 & 0.00 & 0.00 \\
\hline
\end{tabular}




\begin{tabular}{|c|c|c|c|c|c|}
\hline ZFP112 & 236328_PM_at & zinc finger protein 112 homolog (mouse) & -1.32 & 0.00 & 0.00 \\
\hline HLA-DOA & 226878_PM_at & major histocompatibility complex, class II, [ & -1.32 & 0.00 & 0.00 \\
\hline STRBP & 229513_PM_at & spermatid perinuclear RNA binding protein & -1.32 & 0.00 & 0.00 \\
\hline PPM1K & 244011_PM_at & protein phosphatase, $\mathrm{Mg} 2+$ & -1.32 & 0.00 & 0.00 \\
\hline--- & 239673_PM_at & --- & -1.32 & 0.00 & 0.00 \\
\hline COBLL1 & 203642_PM_s_at & COBL-like 1 & -1.32 & 0.00 & 0.00 \\
\hline FCRLA & 235372_PM_at & Fc receptor-like $A$ & -1.32 & 0.00 & 0.00 \\
\hline--- & 241625_PM_at & --- & -1.32 & 0.00 & 0.00 \\
\hline PCDH9 & 219737_PM_s_at & protocadherin 9 & -1.32 & 0.00 & 0.00 \\
\hline--- & 241320_PM_at & --- & -1.31 & 0.00 & 0.00 \\
\hline SLFNL1 & 1553315_PM_at & schlafen-like 1 & -1.31 & 0.00 & 0.00 \\
\hline--- & 1556964_PM_s_at & --- & -1.31 & 0.00 & 0.00 \\
\hline LOC100507539 & 244308_PM_at & uncharacterized LOC100507539 & -1.31 & 0.00 & 0.00 \\
\hline--- & 244878_PM_at & --- & -1.31 & 0.00 & 0.00 \\
\hline--- & 1558410_PM_s_at & --- & -1.31 & 0.00 & 0.00 \\
\hline FAM171A1 & 212771_PM_at & family with sequence similarity 171 , memb & -1.31 & 0.00 & 0.00 \\
\hline AAK1 & 205434_PM_s_at & AP2 associated kinase 1 & -1.31 & 0.00 & 0.00 \\
\hline CAND2 & 213547_PM_at & cullin-associated and neddylation-dissociat & -1.31 & 0.00 & 0.00 \\
\hline MTUS1 & 239576_PM_at & microtubule associated tumor suppressor 1 & -1.31 & 0.00 & 0.00 \\
\hline--- & 232834_PM_at & --- & -1.31 & 0.00 & 0.00 \\
\hline--- & 1560861_PM_at & --- & -1.31 & 0.00 & 0.00 \\
\hline--- & 233302_PM_at & --- & -1.31 & 0.00 & 0.00 \\
\hline--- & 242664_PM_at & --- & -1.31 & 0.00 & 0.00 \\
\hline--- & 237189_PM_at & --- & -1.31 & 0.00 & 0.00 \\
\hline KLHL14 & 228377_PM_at & kelch-like 14 (Drosophila) & -1.31 & 0.00 & 0.01 \\
\hline PPAT & 209433_PM_s_at & phosphoribosyl pyrophosphate amidotrans & -1.31 & 0.00 & 0.01 \\
\hline IGK@ & 215176_PM_x_at & immunoglobulin kappa locus & -1.31 & 0.00 & 0.01 \\
\hline IGK@ & 214768_PM_x_at & immunoglobulin kappa locus & -1.31 & 0.00 & 0.02 \\
\hline IGHA1 & 216510_PM_x_at & immunoglobulin heavy constant alpha 1 & -1.31 & 0.00 & 0.02 \\
\hline BCL11A & 210347_PM_s_at & B-cell CLL & -1.3 & 0.00 & 0.00 \\
\hline TCF4 & 222146_PM_s_at & transcription factor 4 & -1.3 & 0.00 & 0.00 \\
\hline --- & 235756_PM_at & --- & -1.3 & 0.00 & 0.00 \\
\hline --- & 243450_PM_at & --- & -1.3 & 0.00 & 0.00 \\
\hline
\end{tabular}




\begin{tabular}{|c|c|c|c|c|c|}
\hline--- & 241893_PM_at & --- & -1.3 & 0.00 & 0.00 \\
\hline--- & 233995_PM_at & --- & -1.3 & 0.00 & 0.00 \\
\hline--- & 242886_PM_at & --- & -1.3 & 0.00 & 0.00 \\
\hline--- & 241732_PM_at & --- & -1.3 & 0.00 & 0.00 \\
\hline LINC00494 & 236854_PM_at & long intergenic non-protein coding RNA 494 & -1.3 & 0.00 & 0.00 \\
\hline ZCCHC18 & 230224_PM_at & zinc finger, $\mathrm{CCHC}$ domain containing 18 & -1.3 & 0.00 & 0.00 \\
\hline--- & 234822_PM_at & --- & -1.3 & 0.00 & 0.00 \\
\hline DPF3 & 238532_PM_at & D4, zinc and double PHD fingers, family 3 & -1.3 & 0.00 & 0.00 \\
\hline FCRLA & 235400_PM_at & Fc receptor-like $A$ & -1.3 & 0.00 & 0.00 \\
\hline--- & 215284_PM_at & --- & -1.3 & 0.00 & 0.00 \\
\hline CD72 & 215925_PM_s_at & CD72 molecule & -1.3 & 0.00 & 0.00 \\
\hline $\mathrm{CD} 22$ & 204581_PM_at & CD22 molecule & -1.3 & 0.00 & 0.00 \\
\hline--- & 239867_PM_at & --- & -1.3 & 0.00 & 0.01 \\
\hline--- & 235172_PM_at & --- & -1.3 & 0.00 & 0.01 \\
\hline IGK@ & 216576_PM_x_at & immunoglobulin kappa locus & -1.3 & 0.00 & 0.03 \\
\hline--- & 1556932_PM_at & --- & -1.3 & 0.00 & 0.03 \\
\hline CCDC50 & 225331_PM_at & coiled-coil domain containing 50 & -1.29 & 0.00 & 0.00 \\
\hline--- & 1556963_PM_at & --- & -1.29 & 0.00 & 0.00 \\
\hline--- & 243736_PM_at & --- & -1.29 & 0.00 & 0.00 \\
\hline BCL11A & 219498_PM_s_at & B-cell CLL & -1.29 & 0.00 & 0.00 \\
\hline FAM226B & 242292_PM_at & family with sequence similarity 226 , membs & -1.29 & 0.00 & 0.00 \\
\hline PTPRK & 233609_PM_at & Protein tyrosine phosphatase, receptor typ & -1.29 & 0.00 & 0.00 \\
\hline AUTS2 & 212599_PM_at & autism susceptibility candidate 2 & -1.29 & 0.00 & 0.00 \\
\hline--- & 1561195_PM_at & --- & -1.29 & 0.00 & 0.00 \\
\hline--- & 244414_PM_at & --- & -1.29 & 0.00 & 0.00 \\
\hline CD79B & 205297_PM_s_at & CD79b molecule, immunoglobulin-associat $\epsilon$ & -1.29 & 0.00 & 0.00 \\
\hline BANK1 & 1558662_PM_s_at & B-cell scaffold protein with ankyrin repeats & -1.29 & 0.00 & 0.00 \\
\hline--- & 233425_PM_at & --- & -1.29 & 0.00 & 0.00 \\
\hline--- & 243798_PM_at & --- & -1.29 & 0.00 & 0.00 \\
\hline--- & 1562453_PM_at & --- & -1.29 & 0.00 & 0.00 \\
\hline--- & 232614_PM_at & --- & -1.29 & 0.00 & 0.00 \\
\hline MZB1 & 221286_PM_s_at & marginal zone B and B1 cell-specific protein & -1.29 & 0.00 & 0.00 \\
\hline CLDN23 & 228707_PM_at & claudin 23 & -1.29 & 0.00 & 0.00 \\
\hline
\end{tabular}




\begin{tabular}{|c|c|c|c|c|c|}
\hline--- & 233862_PM_at & --- & -1.29 & 0.00 & 0.00 \\
\hline STAP1 & 220059_PM_at & signal transducing adaptor family member: & -1.29 & 0.00 & 0.00 \\
\hline ZNF662 & 228538_PM_at & zinc finger protein 662 & -1.29 & 0.00 & 0.00 \\
\hline--- & 243810_PM_at & --- & -1.29 & 0.00 & 0.00 \\
\hline--- & 224533_PM_s_at & --- & -1.29 & 0.00 & 0.01 \\
\hline--- & 233034_PM_at & --- & -1.29 & 0.00 & 0.01 \\
\hline MS4A1 & 228592_PM_at & membrane-spanning 4-domains, subfamily & -1.29 & 0.00 & 0.01 \\
\hline--- & 1569312_PM_at & --- & -1.29 & 0.00 & 0.01 \\
\hline VPREB3 & 220068_PM_at & pre-B lymphocyte 3 & -1.29 & 0.00 & 0.01 \\
\hline IGHA1 & 215118_PM_s_at & Immunoglobulin heavy constant alpha 1 & -1.29 & 0.00 & 0.02 \\
\hline--- & 236220_PM_at & --- & -1.29 & 0.00 & 0.03 \\
\hline--- & 211645_PM_x_at & --- & -1.29 & 0.00 & 0.03 \\
\hline IGK@ & 211644_PM_x_at & immunoglobulin kappa locus & -1.29 & 0.00 & 0.04 \\
\hline FCRL5 & 224404_PM_s_at & Fc receptor-like 5 & -1.29 & 0.00 & 0.04 \\
\hline IGLC1 & 216853_PM_x_at & Immunoglobulin lambda constant 1 (Mcg m & -1.29 & 0.00 & 0.04 \\
\hline MUM1 & 221290_PM_s_at & melanoma associated antigen (mutated) 1 & -1.28 & 0.00 & 0.00 \\
\hline TCF4 & 203753_PM_at & transcription factor 4 & -1.28 & 0.00 & 0.00 \\
\hline TMEM97 & 212282_PM_at & transmembrane protein 97 & -1.28 & 0.00 & 0.00 \\
\hline TARSL2 & 227611_PM_at & threonyl-tRNA synthetase-like 2 & -1.28 & 0.00 & 0.00 \\
\hline--- & 240605_PM_at & --- & -1.28 & 0.00 & 0.00 \\
\hline--- & 215164_PM_at & --- & -1.28 & 0.00 & 0.00 \\
\hline--- & 235912_PM_at & --- & -1.28 & 0.00 & 0.00 \\
\hline SIK1 & 208078_PM_s_at & salt-inducible kinase 1 & -1.28 & 0.00 & 0.00 \\
\hline METAP1D & 227805_PM_at & methionyl aminopeptidase type 1D (mitoch & -1.28 & 0.00 & 0.00 \\
\hline ZDHHC21 & 229923_PM_at & zinc finger, DHHC-type containing 21 & -1.28 & 0.00 & 0.00 \\
\hline--- & 231450_PM_at & --- & -1.28 & 0.00 & 0.00 \\
\hline LARS & 223888_PM_s_at & leucyl-tRNA synthetase & -1.28 & 0.00 & 0.00 \\
\hline LRPPRC & 1557360_PM_at & leucine-rich pentatricopeptide repeat conta & -1.28 & 0.00 & 0.00 \\
\hline CHD1L & 238070_PM_at & chromodomain helicase DNA binding protei & -1.28 & 0.00 & 0.00 \\
\hline PSMG4 & 242055_PM_at & Proteasome (prosome, macropain) assembl & -1.28 & 0.00 & 0.00 \\
\hline--- & 243855_PM_at & --- & -1.28 & 0.00 & 0.00 \\
\hline--- & 243005_PM_at & --- & -1.28 & 0.00 & 0.00 \\
\hline--- & 236610_PM_at & --- & -1.28 & 0.00 & 0.00 \\
\hline
\end{tabular}




\begin{tabular}{|c|c|c|c|c|c|}
\hline LILRA4 & 210313_PM_at & leukocyte immunoglobulin-like receptor, su & -1.28 & 0.00 & 0.00 \\
\hline SPIB & 205861_PM_at & Spi-B transcription factor (Spi-1 & -1.28 & 0.00 & 0.00 \\
\hline LOC100131541 & 1558569_PM_at & Uncharacterized LOC100131541 & -1.28 & 0.00 & 0.00 \\
\hline SCML1 & 222747_PM_s_at & sex comb on midleg-like 1 (Drosophila) & -1.28 & 0.00 & 0.00 \\
\hline SERPINB9 & 242814_PM_at & serpin peptidase inhibitor, clade B (ovalbun & -1.28 & 0.00 & 0.00 \\
\hline TRAF3IP2 & 202987_PM_at & TRAF3 interacting protein 2 & -1.28 & 0.00 & 0.00 \\
\hline PLEKHA1 & 219024_PM_at & pleckstrin homology domain containing, far & -1.28 & 0.00 & 0.00 \\
\hline RASGRP3 & 205801_PM_s_at & RAS guanyl releasing protein 3 (calcium and & -1.28 & 0.00 & 0.00 \\
\hline--- & 241505_PM_at & --- & -1.28 & 0.00 & 0.00 \\
\hline LINC00342 & 213703_PM_at & long intergenic non-protein coding RNA 342 & -1.28 & 0.00 & 0.00 \\
\hline UBE3D & 1569262_PM_x_at & ubiquitin protein ligase E3D & -1.28 & 0.00 & 0.00 \\
\hline--- & 242741_PM_x_at & --- & -1.28 & 0.00 & 0.00 \\
\hline--- & 236462_PM_at & --- & -1.28 & 0.00 & 0.00 \\
\hline LOC285972 & 1560762_PM_at & uncharacterized LOC285972 & -1.28 & 0.00 & 0.00 \\
\hline LOC100233156 & 227715_PM_at & tektin 4 pseudogene & -1.28 & 0.00 & 0.00 \\
\hline SAMD4A & 212845_PM_at & sterile alpha motif domain containing $4 \mathrm{~A}$ & -1.28 & 0.00 & 0.00 \\
\hline LIX1 & 230865_PM_at & Lix1 homolog (chicken) & -1.28 & 0.00 & 0.01 \\
\hline--- & 1554636_PM_at & --- & -1.28 & 0.00 & 0.01 \\
\hline--- & 234186_PM_at & --- & -1.28 & 0.00 & 0.01 \\
\hline--- & 244181_PM_at & --- & -1.28 & 0.00 & 0.04 \\
\hline CAMTA1 & 213268_PM_at & calmodulin binding transcription activator 1 & -1.28 & 0.00 & 0.04 \\
\hline IGJ & 212592_PM_at & immunoglobulin J polypeptide, linker prote & -1.28 & 0.00 & 0.05 \\
\hline CARD11 & 1562368_PM_at & caspase recruitment domain family, memb & -1.27 & 0.00 & 0.00 \\
\hline U2SURP & 236696_PM_at & U2 snRNP-associated SURP domain contain & -1.27 & 0.00 & 0.00 \\
\hline--- & 240622_PM_at & --- & -1.27 & 0.00 & 0.00 \\
\hline NAF1 & 244828_PM_x_at & nuclear assembly factor 1 homolog (S. cereı & -1.27 & 0.00 & 0.00 \\
\hline AQP3 & 39249_PM_at & aquaporin 3 (Gill blood group) & -1.27 & 0.00 & 0.00 \\
\hline IL6ST & 204863_PM_s_at & interleukin 6 signal transducer (gp130, oncc & -1.27 & 0.00 & 0.00 \\
\hline--- & 237067_PM_at & --- & -1.27 & 0.00 & 0.00 \\
\hline TCF4 & 213891_PM_s_at & transcription factor 4 & -1.27 & 0.00 & 0.00 \\
\hline--- & 244625_PM_at & --- & -1.27 & 0.00 & 0.00 \\
\hline MBNL2 & 232138_PM_at & Muscleblind-like splicing regulator 2 & -1.27 & 0.00 & 0.00 \\
\hline--- & 236279_PM_at & --- & -1.27 & 0.00 & 0.00 \\
\hline
\end{tabular}




\begin{tabular}{|c|c|c|c|c|c|}
\hline METTL6 & 232102_PM_at & Methyltransferase like 6 & -1.27 & 0.00 & 0.00 \\
\hline AQP3 & 203747_PM_at & aquaporin 3 (Gill blood group) & -1.27 & 0.00 & 0.00 \\
\hline TSPAN3 & 200972_PM_at & tetraspanin 3 & -1.27 & 0.00 & 0.00 \\
\hline--- & 232778_PM_at & --- & -1.27 & 0.00 & 0.00 \\
\hline--- & 237953_PM_at & --- & -1.27 & 0.00 & 0.00 \\
\hline PMEPA1 & 222449_PM_at & prostate transmembrane protein, androger & -1.27 & 0.00 & 0.00 \\
\hline EPT1 & 1570371_PM_a_at & ethanolaminephosphotransferase 1 (CDP-e & -1.27 & 0.00 & 0.00 \\
\hline--- & 1560999_PM_a_at & --- & -1.27 & 0.00 & 0.00 \\
\hline NUP62 & 236505_PM_at & Nucleoporin $62 \mathrm{kDa}$ & -1.27 & 0.00 & 0.00 \\
\hline ACAT1 & 1559239_PM_s_at & acetyl-CoA acetyltransferase 1 & -1.27 & 0.00 & 0.0 \\
\hline--- & 1566446_PM_at & --- & -1.27 & 0.00 & 0.00 \\
\hline--- & 243512_PM_x_at & --- & -1.27 & 0.00 & 0.00 \\
\hline--- & 243791_PM_at & --- & -1.27 & 0.00 & 0.00 \\
\hline $\mathrm{BACH} 2$ & 221234_PM_s_at & BTB and CNC homology 1 , basic leucine zipk & -1.27 & 0.00 & 0.00 \\
\hline--- & 244605_PM_at & --- & -1.27 & 0.00 & 0.00 \\
\hline--- & 241417_PM_at & --- & -1.27 & 0.00 & 0.00 \\
\hline--- & 239292_PM_at & --- & -1.27 & 0.00 & 0.00 \\
\hline TTC9 & 213172_PM_at & tetratricopeptide repeat domain 9 & -1.27 & 0.00 & 0.00 \\
\hline SPON1 & 213994_PM_s_at & spondin 1 , extracellular matrix protein & -1.27 & 0.00 & 0.00 \\
\hline AXIN2 & 222696_PM_at & $\operatorname{axin} 2$ & -1.27 & 0.00 & 0.00 \\
\hline $\mathrm{CD} 22$ & 38521_PM_at & CD22 molecule & -1.27 & 0.00 & 0.00 \\
\hline TSPYL5 & 213122_PM_at & TSPY-like 5 & -1.27 & 0.00 & 0.00 \\
\hline--- & 238157_PM_at & --- & -1.27 & 0.00 & 0.00 \\
\hline SNX29 & 232485_PM_at & sorting nexin 29 & -1.27 & 0.00 & 0.00 \\
\hline PTPRK & 203038_PM_at & protein tyrosine phosphatase, receptor typ & -1.27 & 0.00 & 0.01 \\
\hline IGLJ3 & 211798_PM_x_at & immunoglobulin lambda joining 3 & -1.27 & 0.00 & 0.01 \\
\hline RCAN3 & 219864_PM_s_at & RCAN family member 3 & -1.27 & 0.00 & 0.01 \\
\hline USP10 & 209136_PM_s_at & ubiquitin specific peptidase 10 & -1.27 & 0.00 & 0.01 \\
\hline OR2A20P & 222290_PM_at & olfactory receptor, family 2 , subfamily $A, m$ & -1.27 & 0.00 & 0.01 \\
\hline IGLV1-44 & 217258_PM_x_at & immunoglobulin lambda variable 1-44 & -1.27 & 0.00 & 0.02 \\
\hline LOC100507616 & 239244_PM_at & uncharacterized LOC100507616 & -1.27 & 0.00 & 0.02 \\
\hline FCRL5 & 224406_PM_s_at & Fc receptor-like 5 & -1.27 & 0.00 & 0.03 \\
\hline IGLJ3 & 216852_PM_x_at & Immunoglobulin lambda joining 3 & -1.27 & 0.00 & 0.03 \\
\hline
\end{tabular}




\begin{tabular}{|c|c|c|c|c|c|}
\hline IGKC & 216207_PM_x_at & immunoglobulin kappa constant & -1.27 & 0.00 & 0.04 \\
\hline--- & 216401_PM_x_at & --- & -1.27 & 0.00 & 0.04 \\
\hline--- & 217036_PM_at & --- & -1.27 & 0.00 & 0.04 \\
\hline IGKC & 215217_PM_at & Immunoglobulin kappa constant & -1.27 & 0.00 & 0.05 \\
\hline ENAM & 223893_PM_at & enamelin & -1.26 & 0.00 & 0.00 \\
\hline--- & 242920_PM_at & --- & -1.26 & 0.00 & 0.00 \\
\hline--- & 243286_PM_at & --- & -1.26 & 0.00 & 0.00 \\
\hline--- & 232134_PM_at & --- & -1.26 & 0.00 & 0.00 \\
\hline ETS1 & 1555355_PM_a_at & v-ets erythroblastosis virus E26 oncogene $h$ & -1.26 & 0.00 & 0.00 \\
\hline NAA25 & 236816_PM_at & N(alpha)-acetyltransferase 25, NatB auxiliar & -1.26 & 0.00 & 0.00 \\
\hline ZNF275 & 225383_PM_at & zinc finger protein 275 & -1.26 & 0.00 & 0.00 \\
\hline АКT3 & 222880_PM_at & v-akt murine thymoma viral oncogene hom & -1.26 & 0.00 & 0.00 \\
\hline LMLN & 238037_PM_at & leishmanolysin-like (metallopeptidase M8 fi & -1.26 & 0.00 & 0.00 \\
\hline BCL7A & 203796_PM_s_at & B-cell CLL & -1.26 & 0.00 & 0.00 \\
\hline FAM213A & 228155_PM_at & family with sequence similarity 213, membs & -1.26 & 0.00 & 0.00 \\
\hline--- & 240238_PM_at & --- & -1.26 & 0.00 & 0.00 \\
\hline--- & 222266_PM_at & --- & -1.26 & 0.00 & 0.00 \\
\hline HAUS5 & 213054_PM_at & HAUS augmin-like complex, subunit 5 & -1.26 & 0.00 & 0.00 \\
\hline ABLIM1 & 210461_PM_s_at & actin binding LIM protein 1 & -1.26 & 0.00 & 0.00 \\
\hline--- & 241007_PM_at & --- & -1.26 & 0.00 & 0.00 \\
\hline--- & 244845_PM_at & --- & -1.26 & 0.00 & 0.00 \\
\hline C14orf64 & 1559097_PM_at & chromosome 14 open reading frame 64 & -1.26 & 0.00 & 0.00 \\
\hline MAP2K5 & 216765_PM_at & Mitogen-activated protein kinase kinase 5 & -1.26 & 0.00 & 0.00 \\
\hline ZCCHC2 & 219062_PM_s_at & zinc finger, $\mathrm{CCHC}$ domain containing 2 & -1.26 & 0.00 & 0.00 \\
\hline--- & 236343_PM_at & --- & -1.26 & 0.00 & 0.00 \\
\hline SCAF4 & 243759_PM_at & SR-related CTD-associated factor 4 & -1.26 & 0.00 & 0.00 \\
\hline $\mathrm{CD} 27$ & 206150_PM_at & CD27 molecule & -1.26 & 0.00 & 0.00 \\
\hline IKZF1 & 1565818_PM_s_at & IKAROS family zinc finger 1 (Ikaros) & -1.26 & 0.00 & 0.00 \\
\hline SNRPN & 1560741_PM_at & small nuclear ribonucleoprotein polypeptid & -1.26 & 0.00 & 0.00 \\
\hline HTATSF1 & 202602_PM_s_at & HIV-1 Tat specific factor 1 & -1.26 & 0.00 & 0.00 \\
\hline APBA2 & 209871_PM_s_at & amyloid beta (A4) precursor protein-bindin & -1.26 & 0.00 & 0.00 \\
\hline--- & 239784_PM_at & --- & -1.26 & 0.00 & 0.00 \\
\hline DUSP5 & 209457_PM_at & dual specificity phosphatase 5 & -1.26 & 0.00 & 0.00 \\
\hline
\end{tabular}




\begin{tabular}{|c|c|c|c|c|c|}
\hline MLLT3 & 1569652_PM_at & myeloid & -1.26 & 0.00 & 0.00 \\
\hline LOXL1-AS1 & 232752_PM_at & LOXL1 antisense RNA 1 (non-protein coding & -1.26 & 0.00 & 0.00 \\
\hline SLC16A10 & 222939_PM_s_at & solute carrier family 16 , member 10 (aroma & -1.26 & 0.00 & 0.00 \\
\hline TMEM67 & 1569377_PM_at & transmembrane protein 67 & -1.26 & 0.00 & 0.00 \\
\hline DIP2C & 212504_PM_at & DIP2 disco-interacting protein 2 homolog C & -1.26 & 0.00 & 0.00 \\
\hline--- & 217394_PM_at & --- & -1.26 & 0.00 & 0.00 \\
\hline LOC100653018 & 203413_PM_at & uncharacterized LOC100653018 & -1.26 & 0.00 & 0.01 \\
\hline NASP & 242918_PM_at & Nuclear autoantigenic sperm protein (histol & -1.26 & 0.00 & 0.01 \\
\hline TNIP1 & 243423_PM_at & TNFAIP3 interacting protein 1 & -1.26 & 0.00 & 0.01 \\
\hline--- & 1561004_PM_at & --- & -1.26 & 0.00 & 0.02 \\
\hline IGHA1 & 216557_PM_x_at & immunoglobulin heavy constant alpha 1 & -1.26 & 0.00 & 0.03 \\
\hline IGLV1-44 & 234707_PM_x_at & immunoglobulin lambda variable 1-44 & -1.26 & 0.00 & 0.05 \\
\hline ZBTB20 & 205383_PM_s_at & zinc finger and BTB domain containing 20 & -1.25 & 0.00 & 0.00 \\
\hline TGIF2 & 218724_PM_s_at & TGFB-induced factor homeobox 2 & -1.25 & 0.00 & 0.00 \\
\hline NSUN6 & 230200_PM_at & NOP2 & -1.25 & 0.00 & 0.00 \\
\hline--- & 239003_PM_at & --- & -1.25 & 0.00 & 0.00 \\
\hline LUC7L & 1557066_PM_at & LUC7-like (S. cerevisiae) & -1.25 & 0.00 & 0.00 \\
\hline--- & 1567044_PM_s_at & --- & -1.25 & 0.00 & 0.00 \\
\hline ZNF785 & 1554770_PM_x_at & zinc finger protein 785 & -1.25 & 0.00 & 0.00 \\
\hline ATF7IP2 & 219870_PM_at & activating transcription factor 7 interacting & -1.25 & 0.00 & 0.00 \\
\hline--- & 233246_PM_at & --- & -1.25 & 0.00 & 0.00 \\
\hline PRKRA & 237107_PM_at & protein kinase, interferon-inducible double & -1.25 & 0.00 & 0.00 \\
\hline--- & 243030_PM_at & --- & -1.25 & 0.00 & 0.00 \\
\hline SHMT2 & 214095_PM_at & serine hydroxymethyltransferase 2 (mitochı & -1.25 & 0.00 & 0.00 \\
\hline ATAD5 & 220223_PM_at & ATPase family, AAA domain containing 5 & -1.25 & 0.00 & 0.00 \\
\hline--- & 241106_PM_at & --- & -1.25 & 0.00 & 0.00 \\
\hline--- & 222098_PM_s_at & --- & -1.25 & 0.00 & 0.00 \\
\hline ABLIM1 & 200965_PM_s_at & actin binding LIM protein 1 & -1.25 & 0.00 & 0.00 \\
\hline BCL7A & 203795_PM_s_at & B-cell CLL & -1.25 & 0.00 & 0.00 \\
\hline--- & 242031_PM_at & --- & -1.25 & 0.00 & 0.00 \\
\hline TMEM97 & 212281_PM_s_at & transmembrane protein 97 & -1.25 & 0.00 & 0.00 \\
\hline PTPLAD1 & 234000_PM_s_at & protein tyrosine phosphatase-like $\mathrm{A}$ domain & -1.25 & 0.00 & 0.00 \\
\hline--- & 238032_PM_at & --- & -1.25 & 0.00 & 0.00 \\
\hline
\end{tabular}




\begin{tabular}{|c|c|c|c|c|c|}
\hline BCL11A & 219497_PM_s_at & B-cell CLL & -1.25 & 0.00 & 0.00 \\
\hline MAN1C1 & 218918_PM_at & mannosidase, alpha, class $1 \mathrm{C}$, member 1 & -1.25 & 0.00 & 0.00 \\
\hline--- & 215204_PM_at & --- & -1.25 & 0.00 & 0.00 \\
\hline PRICKLE1 & 226065_PM_at & prickle homolog 1 (Drosophila) & -1.25 & 0.00 & 0.00 \\
\hline--- & 233099_PM_at & --- & -1.25 & 0.00 & 0.00 \\
\hline--- & 237429_PM_at & --- & -1.25 & 0.00 & 0.00 \\
\hline--- & 229465_PM_s_at & --- & -1.25 & 0.00 & 0.00 \\
\hline--- & 239862_PM_at & --- & -1.25 & 0.00 & 0.00 \\
\hline--- & 1565614_PM_at & --- & -1.25 & 0.00 & 0.00 \\
\hline HOXB-AS3 & 244512_PM_at & HOXB cluster antisense RNA 3 (non-protein & -1.25 & 0.00 & 0.00 \\
\hline 8-Sep & 209000_PM_s_at & septin 8 & -1.25 & 0.00 & 0.00 \\
\hline BCAS4 & 231584_PM_s_at & Breast carcinoma amplified sequence 4 & -1.25 & 0.00 & 0.00 \\
\hline--- & 240216_PM_at & --- & -1.25 & 0.00 & 0.00 \\
\hline--- & 239966_PM_at & --- & -1.25 & 0.00 & 0.00 \\
\hline STRBP & 233251_PM_at & Spermatid perinuclear RNA binding protein & -1.25 & 0.00 & 0.00 \\
\hline KIAA0664L3 & 238910_PM_at & KIAA0664-like 3 & -1.25 & 0.00 & 0.00 \\
\hline NAPEPLD & 233539_PM_at & $\mathrm{N}$-acyl phosphatidylethanolamine phosphol & -1.25 & 0.00 & 0.00 \\
\hline--- & 236727_PM_at & --- & -1.25 & 0.00 & 0.00 \\
\hline KMO & 205307_PM_s_at & kynurenine 3-monooxygenase (kynurenine & -1.25 & 0.00 & 0.00 \\
\hline--- & 227533_PM_at & --- & -1.25 & 0.00 & 0.00 \\
\hline ARMC8 & 236966_PM_at & armadillo repeat containing 8 & -1.25 & 0.00 & 0.00 \\
\hline--- & 242947_PM_at & --- & -1.25 & 0.00 & 0.00 \\
\hline--- & 231107_PM_at & --- & -1.25 & 0.00 & 0.00 \\
\hline SRRM2 & 1554671_PM_a_at & serine & -1.25 & 0.00 & 0.00 \\
\hline--- & 233921_PM_s_at & --- & -1.25 & 0.00 & 0.00 \\
\hline--- & 240789_PM_at & --- & -1.25 & 0.00 & 0.00 \\
\hline CD40 & 222292_PM_at & CD40 molecule, TNF receptor superfamily $n$ & -1.25 & 0.00 & 0.00 \\
\hline P2RX5 & 210448_PM_s_at & purinergic receptor $\mathrm{P} 2 \mathrm{X}$, ligand-gated ion $\mathrm{cr}$ & -1.25 & 0.00 & 0.00 \\
\hline LOC100129858 & 1556736_PM_at & uncharacterized LOC100129858 & -1.25 & 0.00 & 0.00 \\
\hline CAMTA1 & 1555370_PM_a_at & calmodulin binding transcription activator 1 & -1.25 & 0.00 & 0.00 \\
\hline--- & 238311_PM_at & --- & -1.25 & 0.00 & 0.00 \\
\hline PDE9A & 205593_PM_s_at & phosphodiesterase $9 \mathrm{~A}$ & -1.25 & 0.00 & 0.01 \\
\hline--- & 243006_PM_at & --- & -1.25 & 0.00 & 0.01 \\
\hline
\end{tabular}




\begin{tabular}{|c|c|c|c|c|c|}
\hline SWAP70 & 210369_PM_at & SWAP switching B-cell complex 70kDa subu & -1.25 & 0.00 & 0.01 \\
\hline LOC389834 & 241624_PM_at & ankyrin repeat domain 57 pseudogene & -1.25 & 0.00 & 0.01 \\
\hline IGH@ & 211868_PM_x_at & immunoglobulin heavy locus & -1.25 & 0.00 & 0.01 \\
\hline NT5E & 203939_PM_at & 5'-nucleotidase, ecto (CD73) & -1.25 & 0.00 & 0.01 \\
\hline IGK@ & 211908_PM_x_at & Immunoglobulin kappa locus & -1.25 & 0.00 & 0.01 \\
\hline TPD52 & 201691_PM_s_at & tumor protein D52 & -1.25 & 0.00 & 0.01 \\
\hline CAMK4 & 241871_PM_at & calcium & -1.25 & 0.00 & 0.01 \\
\hline TRABD2A & 227867_PM_at & TraB domain containing $2 \mathrm{~A}$ & -1.25 & 0.00 & 0.01 \\
\hline CDKN1C & 213183_PM_s_at & Cyclin-dependent kinase inhibitor 1C (p57, I & -1.25 & 0.00 & 0.01 \\
\hline LOC100233156 & 229748_PM_x_at & tektin 4 pseudogene & -1.25 & 0.00 & 0.0 \\
\hline CNTNAP2 & 219301_PM_s_at & contactin associated protein-like 2 & -1.25 & 0.00 & 0.03 \\
\hline--- & 1565913_PM_at & --- & -1.25 & 0.00 & 0.03 \\
\hline LOC100287723 & 217480_PM_x_at & ig kappa chain V-I region Walker-like & -1.25 & 0.00 & 0.05 \\
\hline--- & 240438_PM_at & --- & -1.24 & 0.00 & 0.00 \\
\hline ZNF141 & 235580_PM_at & zinc finger protein 141 & -1.24 & 0.00 & 0.00 \\
\hline PEX3 & 203969_PM_at & peroxisomal biogenesis factor 3 & -1.24 & 0.00 & 0.00 \\
\hline--- & 244145_PM_at & --- & -1.24 & 0.00 & 0.00 \\
\hline--- & 236699_PM_at & --- & -1.24 & 0.00 & 0.00 \\
\hline TRIB2 & 202478_PM_at & tribbles homolog 2 (Drosophila) & -1.24 & 0.00 & 0.00 \\
\hline URB1 & 212996_PM_s_at & URB1 ribosome biogenesis 1 homolog (S. c€ & -1.24 & 0.00 & 0.00 \\
\hline--- & 237432_PM_at & --- & -1.24 & 0.00 & 0.00 \\
\hline--- & 234095_PM_at & --- & -1.24 & 0.00 & 0.00 \\
\hline DHX30 & 230871_PM_at & DEAH (Asp-Glu-Ala-His) box polypeptide 30 & -1.24 & 0.00 & 0.00 \\
\hline--- & 244204_PM_at & --- & -1.24 & 0.00 & 0.00 \\
\hline APBA2 & 209870_PM_s_at & amyloid beta (A4) precursor protein-bindin & -1.24 & 0.00 & 0.00 \\
\hline DTWD1 & 236649_PM_at & DTW domain containing 1 & -1.24 & 0.00 & 0.00 \\
\hline--- & 242298_PM_x_at & --- & -1.24 & 0.00 & 0.00 \\
\hline --- & 238653_PM_at & --- & -1.24 & 0.00 & 0.00 \\
\hline--- & 1556345_PM_s_at & --- & -1.24 & 0.00 & 0.00 \\
\hline LCN10 & 238071_PM_at & lipocalin 10 & -1.24 & 0.00 & 0.00 \\
\hline CBX5 & 226085_PM_at & chromobox homolog 5 & -1.24 & 0.00 & 0.00 \\
\hline --- & 243817_PM_at & --- & -1.24 & 0.00 & 0.00 \\
\hline --- & 236143_PM_at & --- & -1.24 & 0.00 & 0.00 \\
\hline
\end{tabular}




\begin{tabular}{|c|c|c|c|c|c|}
\hline--- & 233690_PM_at & --- & -1.24 & 0.00 & 0.00 \\
\hline--- & 1559598_PM_at & --- & -1.24 & 0.00 & 0.00 \\
\hline DPP4 & 203716_PM_s_at & dipeptidyl-peptidase 4 & -1.24 & 0.00 & 0.00 \\
\hline SNRPA1 & 242146_PM_at & Small nuclear ribonucleoprotein polypeptid & -1.24 & 0.00 & 0.00 \\
\hline FAM3C & 236316_PM_at & family with sequence similarity 3, member 1 & -1.24 & 0.00 & 0.00 \\
\hline SEMA6A & 223449_PM_at & sema domain, transmembrane domain (TM & -1.24 & 0.00 & 0.00 \\
\hline--- & 244535_PM_at & --- & -1.24 & 0.00 & 0.00 \\
\hline--- & 230702_PM_at & --- & -1.24 & 0.00 & 0.00 \\
\hline LOC100190986 & 235167_PM_at & uncharacterized LOC100190986 & -1.24 & 0.00 & 0.00 \\
\hline BLK & 206255_PM_at & B lymphoid tyrosine kinase & -1.24 & 0.00 & 0.00 \\
\hline AMIGO1 & 226718_PM_at & adhesion molecule with Ig-like domain 1 & -1.24 & 0.00 & 0.00 \\
\hline--- & 240690_PM_at & --- & -1.24 & 0.00 & 0.00 \\
\hline--- & 236617_PM_at & --- & -1.24 & 0.00 & 0.00 \\
\hline C12orf42 & 1561378_PM_at & chromosome 12 open reading frame 42 & -1.24 & 0.00 & 0.00 \\
\hline--- & 243546_PM_at & --- & -1.24 & 0.00 & 0.00 \\
\hline GPR180 & 1566256_PM_s_at & G protein-coupled receptor 180 & -1.24 & 0.00 & 0.00 \\
\hline--- & 240503_PM_at & --- & -1.24 & 0.00 & 0.00 \\
\hline TCL6 & 239964_PM_at & T-cell leukemia & -1.24 & 0.00 & 0.00 \\
\hline ZNF496 & 238145_PM_at & Zinc finger protein 496 & -1.24 & 0.00 & 0.00 \\
\hline--- & 215802_PM_at & --- & -1.24 & 0.00 & 0.00 \\
\hline--- & 235743_PM_at & --- & -1.24 & 0.00 & 0.01 \\
\hline HNRNPD & 227744_PM_s_at & Heterogeneous nuclear ribonucleoprotein [ & -1.24 & 0.00 & 0.01 \\
\hline ZNF709 & 242028_PM_at & zinc finger protein 709 & -1.24 & 0.00 & 0.01 \\
\hline C11orf80 & 238593_PM_at & chromosome 11 open reading frame 80 & -1.24 & 0.00 & 0.01 \\
\hline COBLL1 & 229598_PM_at & COBL-like 1 & -1.24 & 0.00 & 0.01 \\
\hline SNX22 & 230509_PM_at & sorting nexin 22 & -1.24 & 0.00 & 0.01 \\
\hline--- & 217536_PM_x_at & --- & -1.24 & 0.00 & 0.01 \\
\hline ELOVL4 & 219532_PM_at & ELOVL fatty acid elongase 4 & -1.24 & 0.00 & 0.01 \\
\hline--- & 217251_PM_x_at & --- & -1.24 & 0.00 & 0.02 \\
\hline KIAA0125 & 206478_PM_at & KIAA0125 & -1.24 & 0.00 & 0.02 \\
\hline GCOM1 & 228568_PM_at & GRINL1A complex locus 1 & -1.24 & 0.00 & 0.02 \\
\hline--- & 239448_PM_at & --- & -1.24 & 0.00 & 0.04 \\
\hline IGH@ & 217281_PM_x_at & immunoglobulin heavy locus & -1.24 & 0.00 & 0.05 \\
\hline
\end{tabular}




\begin{tabular}{|c|c|c|c|c|c|}
\hline ZNF202 & 204327_PM_s_at & zinc finger protein 202 & -1.23 & 0.00 & 0.00 \\
\hline--- & 222306_PM_at & --- & -1.23 & 0.00 & 0.00 \\
\hline LUC7L & 1557067_PM_s_at & LUC7-like (S. cerevisiae) & -1.23 & 0.00 & 0.00 \\
\hline--- & 236139_PM_at & --- & -1.23 & 0.00 & 0.00 \\
\hline--- & 239397_PM_at & --- & -1.23 & 0.00 & 0.00 \\
\hline--- & 215526_PM_at & --- & -1.23 & 0.00 & 0.00 \\
\hline MPRIP & 214694_PM_at & myosin phosphatase Rho interacting proteiı & -1.23 & 0.00 & 0.00 \\
\hline--- & 1560680_PM_at & --- & -1.23 & 0.00 & 0.00 \\
\hline MORC4 & 219038_PM_at & MORC family CW-type zinc finger 4 & -1.23 & 0.00 & 0.00 \\
\hline GART & 244822_PM_at & phosphoribosylglycinamide formyltransfera & -1.23 & 0.00 & 0.00 \\
\hline TGFBR1 & 236561_PM_at & transforming growth factor, beta receptor 1 & -1.23 & 0.00 & 0.00 \\
\hline--- & 1567045_PM_at & --- & -1.23 & 0.00 & 0.00 \\
\hline TNPO1 & 1556116_PM_s_at & Transportin 1 & -1.23 & 0.00 & 0.00 \\
\hline MMP19 & 204574_PM_s_at & matrix metallopeptidase 19 & -1.23 & 0.00 & 0.00 \\
\hline TRIB2 & 202479_PM_s_at & tribbles homolog 2 (Drosophila) & -1.23 & 0.00 & 0.00 \\
\hline C14orf64 & 1564211_PM_at & chromosome 14 open reading frame 64 & -1.23 & 0.00 & 0.00 \\
\hline GPR18 & 210279_PM_at & G protein-coupled receptor 18 & -1.23 & 0.00 & 0.00 \\
\hline--- & 236210_PM_at & --- & -1.23 & 0.00 & 0.00 \\
\hline--- & 238106_PM_at & --- & -1.23 & 0.00 & 0.00 \\
\hline--- & 1554771_PM_at & --- & -1.23 & 0.00 & 0.00 \\
\hline TACR1 & 210637_PM_at & tachykinin receptor 1 & -1.23 & 0.00 & 0.00 \\
\hline LOC100653004 & 242490_PM_at & uncharacterized LOC100653004 & -1.23 & 0.00 & 0.00 \\
\hline ARL10 & 228843_PM_at & ADP-ribosylation factor-like 10 & -1.23 & 0.00 & 0.00 \\
\hline--- & 1562529_PM_s_at & --- & -1.23 & 0.00 & 0.00 \\
\hline HBS1L & 209315_PM_at & HBS1-like (S. cerevisiae) & -1.23 & 0.00 & 0.00 \\
\hline TAF4B & 235020_PM_at & TAF4b RNA polymerase II, TATA box binding & -1.23 & 0.00 & 0.00 \\
\hline--- & 220728_PM_at & --- & -1.23 & 0.00 & 0.00 \\
\hline CAMSAP1 & 244682_PM_at & calmodulin regulated spectrin-associated pı & -1.23 & 0.00 & 0.00 \\
\hline--- & 1565886_PM_at & --- & -1.23 & 0.00 & 0.00 \\
\hline LOC100291323 & 1564639_PM_at & uncharacterized LOC100291323 & -1.23 & 0.00 & 0.00 \\
\hline--- & 242752_PM_at & --- & -1.23 & 0.00 & 0.00 \\
\hline--- & 1564776_PM_at & --- & -1.23 & 0.00 & 0.00 \\
\hline SERPINF1 & 202283_PM_at & serpin peptidase inhibitor, clade F (alpha-2 & -1.23 & 0.00 & 0.00 \\
\hline
\end{tabular}




\begin{tabular}{|c|c|c|c|c|c|}
\hline--- & 244633_PM_at & --- & -1.23 & 0.00 & 0.00 \\
\hline--- & 234848_PM_at & --- & -1.23 & 0.00 & 0.00 \\
\hline--- & 1555157_PM_at & --- & -1.23 & 0.00 & 0.00 \\
\hline--- & 242409_PM_at & --- & -1.23 & 0.00 & 0.00 \\
\hline--- & 240971_PM_x_at & --- & -1.23 & 0.00 & 0.00 \\
\hline--- & 241298_PM_x_at & --- & -1.23 & 0.00 & 0.00 \\
\hline RGCC & 239827_PM_at & Regulator of cell cycle & -1.23 & 0.00 & 0.00 \\
\hline YME1L1 & 234849_PM_at & YME1-like 1 (S. cerevisiae) & -1.23 & 0.00 & 0.01 \\
\hline--- & 1566607_PM_at & --- & -1.23 & 0.00 & 0.01 \\
\hline PGAP1 & 220576_PM_at & post-GPI attachment to proteins 1 & -1.23 & 0.00 & 0.01 \\
\hline--- & 244226_PM_s_at & --- & -1.23 & 0.00 & 0.01 \\
\hline--- & 236149_PM_at & --- & -1.23 & 0.00 & 0.01 \\
\hline CCDC91 & 1570571_PM_at & Coiled-coil domain containing 91 & -1.23 & 0.00 & 0.01 \\
\hline--- & 235268_PM_at & --- & -1.23 & 0.00 & 0.01 \\
\hline KMO & 211138_PM_s_at & kynurenine 3-monooxygenase (kynurenine & -1.23 & 0.00 & 0.01 \\
\hline--- & 239588_PM_s_at & --- & -1.23 & 0.00 & 0.01 \\
\hline SLFN5 & 1557078_PM_at & schlafen family member 5 & -1.23 & 0.00 & 0.01 \\
\hline--- & 241092_PM_at & --- & -1.23 & 0.00 & 0.01 \\
\hline--- & 216173_PM_at & --- & -1.23 & 0.00 & 0.01 \\
\hline SLC16A7 & 207057_PM_at & solute carrier family 16 , member 7 (monoca & -1.23 & 0.00 & 0.01 \\
\hline MYEF2 & 229464_PM_at & myelin expression factor 2 & -1.23 & 0.00 & 0.01 \\
\hline PCNP & 237577_PM_at & PEST proteolytic signal containing nuclear $p$ & -1.23 & 0.00 & 0.01 \\
\hline KMO & 205306_PM_x_at & kynurenine 3-monooxygenase (kynurenine & -1.23 & 0.00 & 0.02 \\
\hline IGHG1 & 228518_PM_at & immunoglobulin heavy constant gamma 1 ( & -1.23 & 0.00 & 0.02 \\
\hline FAM129C & 1553369_PM_at & family with sequence similarity 129 , memb & -1.23 & 0.00 & 0.03 \\
\hline--- & 239278_PM_at & --- & -1.23 & 0.00 & 0.03 \\
\hline IGLC1 & 209138_PM_x_at & Immunoglobulin lambda constant 1 (Mcg m & -1.23 & 0.00 & 0.03 \\
\hline WDR4 & 235551_PM_at & WD repeat domain 4 & -1.23 & 0.00 & 0.03 \\
\hline--- & 242836_PM_at & --- & -1.23 & 0.00 & 0.03 \\
\hline IGLJ3 & 211881_PM_x_at & immunoglobulin lambda joining 3 & -1.23 & 0.00 & 0.03 \\
\hline--- & 1563364_PM_at & --- & -1.23 & 0.00 & 0.03 \\
\hline--- & 216495_PM_x_at & --- & -1.23 & 0.00 & 0.05 \\
\hline C100505549 & 238055_PM_at & uncharacterized LOC100505549 & -1.22 & 0.00 & 0.00 \\
\hline
\end{tabular}




\begin{tabular}{|c|c|c|c|c|c|}
\hline CBFA2T2 & 238549_PM_at & core-binding factor, runt domain, alpha sub & -1.22 & 0.00 & 0.00 \\
\hline--- & 226773_PM_at & --- & -1.22 & 0.00 & 0.00 \\
\hline--- & 236484_PM_at & --- & -1.22 & 0.00 & 0.00 \\
\hline--- & 242089_PM_at & --- & -1.22 & 0.00 & 0.00 \\
\hline--- & 236330_PM_at & --- & -1.22 & 0.00 & 0.00 \\
\hline HSPH1 & 208744_PM_x_at & heat shock $105 \mathrm{kDa}$ & -1.22 & 0.00 & 0.00 \\
\hline--- & 1556126_PM_s_at & --- & -1.22 & 0.00 & 0.00 \\
\hline CLN8 & 219341_PM_at & ceroid-lipofuscinosis, neuronal 8 (epilepsy, | & -1.22 & 0.00 & 0.00 \\
\hline ZNF827 & 226764_PM_at & zinc finger protein 827 & -1.22 & 0.00 & 0.00 \\
\hline SPTAN1 & 214925_PM_s_at & spectrin, alpha, non-erythrocytic 1 & -1.22 & 0.00 & 0.00 \\
\hline--- & 1569597_PM_at & --- & -1.22 & 0.00 & 0.00 \\
\hline FAIM3 & 221602_PM_s_at & Fas apoptotic inhibitory molecule 3 & -1.22 & 0.00 & 0.00 \\
\hline--- & 218544_PM_s_at & --- & -1.22 & 0.00 & 0.00 \\
\hline GSPT1 & 240452_PM_at & G1 to $S$ phase transition 1 & -1.22 & 0.00 & 0.00 \\
\hline ATXN2L & 207798_PM_s_at & ataxin 2-like & -1.22 & 0.00 & 0.00 \\
\hline AQP3 & 39248_PM_at & aquaporin 3 (Gill blood group) & -1.22 & 0.00 & 0.00 \\
\hline EIF3B & 236274_PM_at & eukaryotic translation initiation factor 3 , sul & -1.22 & 0.00 & 0.00 \\
\hline SEL1L3 & 212314_PM_at & sel-1 suppressor of lin-12-like 3 (C. elegans) & -1.22 & 0.00 & 0.00 \\
\hline GOLGA8A & 208797_PM_s_at & golgin $A 8$ family, member $A$ & -1.22 & 0.00 & 0.00 \\
\hline LOC100128108 & 243957_PM_at & uncharacterized LOC100128108 & -1.22 & 0.00 & 0.00 \\
\hline--- & 241750_PM_x_at & --- & -1.22 & 0.00 & 0.00 \\
\hline LOC100507053 & 1559240_PM_at & uncharacterized LOC100507053 & -1.22 & 0.00 & 0.00 \\
\hline--- & 239348_PM_at & --- & -1.22 & 0.00 & 0.00 \\
\hline LOC100133130 & 223766_PM_at & PRO1102 & -1.22 & 0.00 & 0.00 \\
\hline RNF157-AS1 & 230776_PM_at & RNF157 antisense RNA 1 (non-protein codir & -1.22 & 0.00 & 0.00 \\
\hline IL21R & 219971_PM_at & interleukin 21 receptor & -1.22 & 0.00 & 0.00 \\
\hline FAIM3 & 221601_PM_s_at & Fas apoptotic inhibitory molecule 3 & -1.22 & 0.00 & 0.00 \\
\hline--- & 238098_PM_at & --- & -1.22 & 0.00 & 0.00 \\
\hline C16orf46 & 230281_PM_at & chromosome 16 open reading frame 46 & -1.22 & 0.00 & 0.00 \\
\hline SEC62 & 1552789_PM_at & SEC62 homolog (S. cerevisiae) & -1.22 & 0.00 & 0.00 \\
\hline CXCR7 & 212977_PM_at & chemokine ( $\mathrm{C}-\mathrm{X}-\mathrm{C}$ motif) receptor 7 & -1.22 & 0.00 & 0.00 \\
\hline CAMK2D & 224994_PM_at & calcium & -1.22 & 0.00 & 0.00 \\
\hline OPN3 & 224392_PM_s_at & opsin 3 & -1.22 & 0.00 & 0.00 \\
\hline
\end{tabular}




\begin{tabular}{|c|c|c|c|c|c|}
\hline--- & 242709_PM_s_at & --- & -1.22 & 0.00 & 0.00 \\
\hline--- & 234548_PM_at & --- & -1.22 & 0.00 & 0.00 \\
\hline CYP3A5 & 214235_PM_at & cytochrome $\mathrm{P} 450$, family 3 , subfamily A, po & -1.22 & 0.00 & 0.00 \\
\hline $\mathrm{RHOH}$ & 236293_PM_at & ras homolog family member $\mathrm{H}$ & -1.22 & 0.00 & 0.00 \\
\hline LOC100506639 & 1557384_PM_at & uncharacterized LOC100506639 & -1.22 & 0.00 & 0.00 \\
\hline PIGL & 232262_PM_at & phosphatidylinositol glycan anchor biosyntr & -1.22 & 0.00 & 0.00 \\
\hline--- & 239842_PM_x_at & --- & -1.22 & 0.00 & 0.00 \\
\hline SUN1 & 206487_PM_at & Sad1 and UNC84 domain containing 1 & -1.22 & 0.00 & 0.00 \\
\hline--- & 1569601_PM_at & --- & -1.22 & 0.00 & 0.00 \\
\hline ZMAT4 & 219877_PM_at & zinc finger, matrin-type 4 & -1.22 & 0.00 & 0.00 \\
\hline AKAP13 & 229783_PM_at & A kinase (PRKA) anchor protein 13 & -1.22 & 0.00 & 0.00 \\
\hline--- & 236395_PM_at & --- & -1.22 & 0.00 & 0.00 \\
\hline RALGAPA1 & 234923_PM_at & Ral GTPase activating protein, alpha subuni & -1.22 & 0.00 & 0.00 \\
\hline ZNF83 & 236429_PM_at & zinc finger protein 83 & -1.22 & 0.00 & 0.00 \\
\hline HELLS & 227350_PM_at & helicase, lymphoid-specific & -1.22 & 0.00 & 0.00 \\
\hline RBM6 & 1556672_PM_a_at & RNA binding motif protein 6 & -1.22 & 0.00 & 0.00 \\
\hline--- & 233614_PM_at & --- & -1.22 & 0.00 & 0.00 \\
\hline--- & 1557555_PM_at & --- & -1.22 & 0.00 & 0.01 \\
\hline FOSB & 202768_PM_at & FBJ murine osteosarcoma viral oncogene hc & -1.22 & 0.00 & 0.01 \\
\hline--- & 217697_PM_at & --- & -1.22 & 0.00 & 0.01 \\
\hline--- & 244239_PM_at & --- & -1.22 & 0.00 & 0.01 \\
\hline--- & 244053_PM_at & --- & -1.22 & 0.00 & 0.01 \\
\hline--- & 243089_PM_at & --- & -1.22 & 0.00 & 0.01 \\
\hline--- & 243416_PM_at & --- & -1.22 & 0.00 & 0.01 \\
\hline--- & 242225_PM_at & --- & -1.22 & 0.00 & 0.01 \\
\hline RAB30 & 228390_PM_at & RAB30, member RAS oncogene family & -1.22 & 0.00 & 0.01 \\
\hline--- & 244665_PM_at & --- & -1.22 & 0.00 & 0.01 \\
\hline SHISA4 & 226674_PM_at & shisa homolog 4 (Xenopus laevis) & -1.22 & 0.00 & 0.01 \\
\hline--- & 244331_PM_at & --- & -1.22 & 0.00 & 0.01 \\
\hline NAV1 & 242986_PM_at & Neuron navigator 1 & -1.22 & 0.00 & 0.01 \\
\hline EPHX2 & 209368_PM_at & epoxide hydrolase 2 , cytoplasmic & -1.22 & 0.00 & 0.01 \\
\hline--- & 216211_PM_at & --- & -1.22 & 0.00 & 0.01 \\
\hline--- & 1559188_PM_x_at & --- & -1.22 & 0.00 & 0.01 \\
\hline
\end{tabular}




\begin{tabular}{|c|c|c|c|c|c|}
\hline YTHDC1 & 214814_PM_at & YTH domain containing 1 & -1.22 & 0.00 & 0.01 \\
\hline GART & 230766_PM_at & phosphoribosylglycinamide formyltransfera & -1.22 & 0.00 & 0.01 \\
\hline HIST1H2AB & 214469_PM_at & histone cluster $1, \mathrm{H} 2 \mathrm{ab}$ & -1.22 & 0.00 & 0.01 \\
\hline GOSR2 & 243880_PM_at & golgi SNAP receptor complex member 2 & -1.22 & 0.00 & 0.01 \\
\hline FMNL3 & 238823_PM_at & formin-like 3 & -1.22 & 0.00 & 0.01 \\
\hline--- & 232835_PM_at & --- & -1.22 & 0.00 & 0.02 \\
\hline CYAT1 & 215121_PM_x_at & immunoglobulin lambda light chain-like & -1.22 & 0.00 & 0.02 \\
\hline--- & 241391_PM_at & --- & -1.22 & 0.00 & 0.02 \\
\hline--- & 240442_PM_at & --- & -1.22 & 0.00 & 0.02 \\
\hline CD28 & 206545_PM_at & CD28 molecule & -1.22 & 0.00 & 0.03 \\
\hline IL23A & 234865_PM_at & Interleukin 23, alpha subunit p19 & -1.22 & 0.00 & 0.03 \\
\hline--- & 1556375_PM_at & --- & -1.21 & 0.00 & 0.00 \\
\hline GAR1 & 219110_PM_at & GAR1 ribonucleoprotein homolog (yeast) & -1.21 & 0.00 & 0.00 \\
\hline PCSK7 & 232521_PM_at & proprotein convertase subtilisin & -1.21 & 0.00 & 0.00 \\
\hline PSMG4 & 228217_PM_s_at & proteasome (prosome, macropain) assembl & -1.21 & 0.00 & 0.00 \\
\hline ZNF785 & 1554769_PM_at & zinc finger protein 785 & -1.21 & 0.00 & 0.00 \\
\hline$A D C$ & 228000_PM_at & arginine decarboxylase & -1.21 & 0.00 & 0.00 \\
\hline WDR61 & 215156_PM_at & WD repeat domain 61 & -1.21 & 0.00 & 0.00 \\
\hline QSOX2 & 227146_PM_at & quiescin Q6 sulfhydryl oxidase 2 & -1.21 & 0.00 & 0.00 \\
\hline--- & 239238_PM_at & --- & -1.21 & 0.00 & 0.00 \\
\hline LOC100507495 & 236520_PM_at & Uncharacterized LOC100507495 & -1.21 & 0.00 & 0.00 \\
\hline--- & 233243_PM_at & --- & -1.21 & 0.00 & 0.00 \\
\hline ELP2 & 235623_PM_at & elongation protein 2 homolog (S. cerevisiae & -1.21 & 0.00 & 0.00 \\
\hline DGCR8 & 219811_PM_at & DiGeorge syndrome critical region gene 8 & -1.21 & 0.00 & 0.00 \\
\hline--- & 1561640_PM_at & --- & -1.21 & 0.00 & 0.00 \\
\hline--- & 241472_PM_at & --- & -1.21 & 0.00 & 0.00 \\
\hline cox18 & 227442_PM_at & COX18 cytochrome c oxidase assembly hom & -1.21 & 0.00 & 0.00 \\
\hline SNHG12 & 223773_PM_s_at & small nucleolar RNA host gene 12 (non-prot & -1.21 & 0.00 & 0.00 \\
\hline SDAD1 & 218607_PM_s_at & SDA1 domain containing 1 & -1.21 & 0.00 & 0.00 \\
\hline --- & 241930_PM_x_at & --- & -1.21 & 0.00 & 0.00 \\
\hline RGS12 & 242079_PM_at & regulator of G-protein signaling 12 & -1.21 & 0.00 & 0.00 \\
\hline RIMKLB & 226164_PM_x_at & ribosomal modification protein rimK-like faı & -1.21 & 0.00 & 0.00 \\
\hline MTERFD2 & 1557965_PM_at & MTERF domain containing 2 & -1.21 & 0.00 & 0.00 \\
\hline
\end{tabular}




\begin{tabular}{|c|c|c|c|c|c|}
\hline NOP14 & 214661_PM_s_at & NOP14 nucleolar protein homolog (yeast) & -1.21 & 0.00 & 0.00 \\
\hline ANAPC5 & 239651_PM_at & anaphase promoting complex subunit 5 & -1.21 & 0.00 & 0.00 \\
\hline--- & 1557300_PM_s_at & --- & -1.21 & 0.00 & 0.00 \\
\hline NGDN & 216263_PM_s_at & neuroguidin, EIF4E binding protein & -1.21 & 0.00 & 0.00 \\
\hline--- & 222284_PM_at & --- & -1.21 & 0.00 & 0.00 \\
\hline CEP41 & 225484_PM_at & centrosomal protein $41 \mathrm{kDa}$ & -1.21 & 0.00 & 0.00 \\
\hline--- & 233648_PM_at & --- & -1.21 & 0.00 & 0.00 \\
\hline--- & 239070_PM_at & --- & -1.21 & 0.00 & 0.00 \\
\hline HNRNPA1 & 222040_PM_at & heterogeneous nuclear ribonucleoprotein $A$ & -1.21 & 0.00 & 0.00 \\
\hline EVL & 227232_PM_at & Enah & -1.21 & 0.00 & 0.00 \\
\hline FAM208B & 244165_PM_at & family with sequence similarity 208, membt & -1.21 & 0.00 & 0.00 \\
\hline--- & 241489_PM_at & --- & -1.21 & 0.00 & 0.00 \\
\hline HNRNPA1 & 214280_PM_x_at & heterogeneous nuclear ribonucleoprotein $A$ & -1.21 & 0.00 & 0.00 \\
\hline--- & 242108_PM_at & --- & -1.21 & 0.00 & 0.00 \\
\hline CUL4A & 232466_PM_at & Cullin 4A & -1.21 & 0.00 & 0.00 \\
\hline C5orf63 & 244761_PM_at & chromosome 5 open reading frame 63 & -1.21 & 0.00 & 0.00 \\
\hline SPATS2 & 222593_PM_s_at & spermatogenesis associated, serine-rich 2 & -1.21 & 0.00 & 0.00 \\
\hline MAP3К9 & 213927_PM_at & mitogen-activated protein kinase kinase kin & -1.21 & 0.00 & 0.00 \\
\hline--- & 237388_PM_at & --- & -1.21 & 0.00 & 0.00 \\
\hline TCF12 & 215611_PM_at & transcription factor 12 & -1.21 & 0.00 & 0.00 \\
\hline SEMA4C & 46665_PM_at & sema domain, immunoglobulin domain (Ig), & -1.21 & 0.00 & 0.00 \\
\hline LY9 & 231124_PM_x_at & lymphocyte antigen 9 & -1.21 & 0.00 & 0.00 \\
\hline FAM69A & 1556498_PM_at & Family with sequence similarity 69, membe & -1.21 & 0.00 & 0.00 \\
\hline--- & 241435_PM_at & --- & -1.21 & 0.00 & 0.00 \\
\hline--- & 243280_PM_at & --- & -1.21 & 0.00 & 0.00 \\
\hline ZMYM6 & 1552969_PM_a_at & zinc finger, MYM-type 6 & -1.21 & 0.00 & 0.00 \\
\hline WDR60 & 229008_PM_at & WD repeat domain 60 & -1.21 & 0.00 & 0.00 \\
\hline--- & 235788_PM_at & --- & -1.21 & 0.00 & 0.00 \\
\hline--- & 237216_PM_at & --- & -1.21 & 0.00 & 0.00 \\
\hline ARHGEF26 & 233903_PM_s_at & Rho guanine nucleotide exchange factor (G) & -1.21 & 0.00 & 0.00 \\
\hline--- & 233444_PM_at & --- & -1.21 & 0.00 & 0.00 \\
\hline LOC100506930 & 243924_PM_at & uncharacterized LOC100506930 & -1.21 & 0.00 & 0.00 \\
\hline AKT3 & 242876_PM_at & V-akt murine thymoma viral oncogene hom & -1.21 & 0.00 & 0.00 \\
\hline
\end{tabular}




\begin{tabular}{|c|c|c|c|c|c|}
\hline RRN3P1 & 216908_PM_x_at & RNA polymerase I transcription factor homc & -1.21 & 0.00 & 0.00 \\
\hline CD83 & 204440_PM_at & CD83 molecule & -1.21 & 0.00 & 0.00 \\
\hline GAL3ST4 & 219815_PM_at & galactose-3-O-sulfotransferase 4 & -1.21 & 0.00 & 0.00 \\
\hline PIK3C2B & 204484_PM_at & phosphoinositide-3-kinase, class 2, beta pol & -1.21 & 0.00 & 0.00 \\
\hline CYP2B6 & 206754_PM_s_at & cytochrome P450, family 2 , subfamily B, pol & -1.21 & 0.00 & 0.00 \\
\hline--- & 234785_PM_at & --- & -1.21 & 0.00 & 0.00 \\
\hline--- & 232978_PM_at & --- & -1.21 & 0.00 & 0.00 \\
\hline CAND1 & 239771_PM_at & cullin-associated and neddylation-dissociat & -1.21 & 0.00 & 0.00 \\
\hline KLHL3 & 221221_PM_s_at & kelch-like 3 (Drosophila) & -1.21 & 0.00 & 0.00 \\
\hline--- & 233706_PM_at & --- & -1.21 & 0.00 & 0.00 \\
\hline--- & 239868_PM_at & --- & -1.21 & 0.00 & 0.00 \\
\hline--- & 238536_PM_at & --- & -1.21 & 0.00 & 0.00 \\
\hline NBPF10 & 242191_PM_at & neuroblastoma breakpoint family, member & -1.21 & 0.00 & 0.00 \\
\hline RORA & 235567_PM_at & RAR-related orphan receptor $A$ & -1.21 & 0.00 & 0.01 \\
\hline--- & 241079_PM_at & --- & -1.21 & 0.00 & 0.01 \\
\hline--- & 235813_PM_at & --- & -1.21 & 0.00 & 0.01 \\
\hline--- & 1570329_PM_at & --- & -1.21 & 0.00 & 0.01 \\
\hline--- & 1569499_PM_at & --- & -1.21 & 0.00 & 0.01 \\
\hline--- & 1559425_PM_at & --- & -1.21 & 0.00 & 0.01 \\
\hline LOC283174 & 229734_PM_at & uncharacterized LOC283174 & -1.21 & 0.00 & 0.01 \\
\hline CXCR5 & 206126_PM_at & chemokine ( $\mathrm{C}-\mathrm{X}-\mathrm{C}$ motif) receptor 5 & -1.21 & 0.00 & 0.01 \\
\hline--- & 243473_PM_at & --- & -1.21 & 0.00 & 0.01 \\
\hline KLHDC4 & 234738_PM_s_at & kelch domain containing 4 & -1.21 & 0.00 & 0.01 \\
\hline CELSR3 & 205165_PM_at & cadherin, EGF LAG seven-pass G-type recep & -1.21 & 0.00 & 0.01 \\
\hline ABCB4 & 207819_PM_s_at & ATP-binding cassette, sub-family B (MDR & -1.21 & 0.00 & 0.01 \\
\hline--- & 238155_PM_at & --- & -1.21 & 0.00 & 0.01 \\
\hline FCRL2 & 221239_PM_s_at & Fc receptor-like 2 & -1.21 & 0.00 & 0.01 \\
\hline--- & 233497_PM_at & --- & -1.21 & 0.00 & 0.01 \\
\hline IGK@ & 214669_PM_x_at & immunoglobulin kappa locus & -1.21 & 0.00 & 0.01 \\
\hline TBC1D4 & 203386_PM_at & TBC1 domain family, member 4 & -1.21 & 0.00 & 0.01 \\
\hline DPP4 & 211478_PM_s_at & dipeptidyl-peptidase 4 & -1.21 & 0.00 & 0.01 \\
\hline --- & 244379_PM_at & --- & -1.21 & 0.00 & 0.01 \\
\hline--- & 229327_PM_s_at & --- & -1.21 & 0.00 & 0.01 \\
\hline
\end{tabular}




\begin{tabular}{|c|c|c|c|c|c|}
\hline BANK1 & 222915_PM_s_at & B-cell scaffold protein with ankyrin repeats & -1.21 & 0.00 & 0.02 \\
\hline HELB & 1552787_PM_at & helicase (DNA) B & -1.21 & 0.00 & 0.02 \\
\hline BTLA & 236226_PM_at & $\mathrm{B}$ and T Iymphocyte associated & -1.21 & 0.00 & 0.02 \\
\hline MIR600 & 223522_PM_at & microRNA 600 & -1.21 & 0.00 & 0.02 \\
\hline TCF4 & 212385_PM_at & transcription factor 4 & -1.21 & 0.00 & 0.02 \\
\hline--- & 239227_PM_at & --- & -1.21 & 0.00 & 0.02 \\
\hline SDC2 & 212158_PM_at & syndecan 2 & -1.21 & 0.00 & 0.02 \\
\hline--- & 1563706_PM_at & --- & -1.21 & 0.00 & 0.02 \\
\hline--- & 1557437_PM_a_at & --- & -1.21 & 0.00 & 0.02 \\
\hline IGLV1-44 & 215379_PM_x_at & immunoglobulin lambda variable 1-44 & -1.21 & 0.00 & 0.02 \\
\hline--- & 232882_PM_at & --- & -1.21 & 0.00 & 0.03 \\
\hline EPHA1-AS1 & 1562249_PM_at & EPHA1 antisense RNA 1 (non-protein codin६ & -1.21 & 0.00 & 0.03 \\
\hline KIAA0355 & 203288_PM_at & KIAA0355 & -1.2 & 0.00 & 0.00 \\
\hline RBM17 & 224781_PM_s_at & RNA binding motif protein 17 & -1.2 & 0.00 & 0.00 \\
\hline ZNF37A & 228711_PM_at & zinc finger protein $37 \mathrm{~A}$ & -1.2 & 0.00 & 0.00 \\
\hline CSTF3 & 229665_PM_at & cleavage stimulation factor, 3' pre-RNA, suk & -1.2 & 0.00 & 0.00 \\
\hline U2SURP & 236431_PM_at & U2 snRNP-associated SURP domain contain & -1.2 & 0.00 & 0.00 \\
\hline KTN1 & 200914_PM_x_at & kinectin 1 (kinesin receptor) & -1.2 & 0.00 & 0.00 \\
\hline ZBTB4 & 225629_PM_s_at & zinc finger and BTB domain containing 4 & -1.2 & 0.00 & 0.00 \\
\hline--- & 242194_PM_at & --- & -1.2 & 0.00 & 0.00 \\
\hline DCAF8 & 243318_PM_at & DDB1 and CUL4 associated factor 8 & -1.2 & 0.00 & 0.00 \\
\hline FKBP4 & 200895_PM_s_at & FK506 binding protein $4,59 \mathrm{kDa}$ & -1.2 & 0.00 & 0.00 \\
\hline--- & 240595_PM_at & --- & -1.2 & 0.00 & 0.00 \\
\hline SFXN4 & 230637_PM_at & sideroflexin 4 & -1.2 & 0.00 & 0.00 \\
\hline--- & 242448_PM_at & --- & -1.2 & 0.00 & 0.00 \\
\hline MTERFD2 & 1557966_PM_x_at & MTERF domain containing 2 & -1.2 & 0.00 & 0.00 \\
\hline PTCD2 & 219658_PM_at & pentatricopeptide repeat domain 2 & -1.2 & 0.00 & 0.00 \\
\hline SLC20A1 & 230494_PM_at & solute carrier family 20 (phosphate transpo & -1.2 & 0.00 & 0.00 \\
\hline--- & 236379_PM_at & --- & -1.2 & 0.00 & 0.00 \\
\hline MSI2 & 225240_PM_s_at & musashi homolog 2 (Drosophila) & -1.2 & 0.00 & 0.00 \\
\hline SERBP1 & 209669_PM_s_at & SERPINE1 mRNA binding protein 1 & -1.2 & 0.00 & 0.00 \\
\hline RBM19 & 206019_PM_at & RNA binding motif protein 19 & -1.2 & 0.00 & 0.00 \\
\hline--- & 217647_PM_at & --- & -1.2 & 0.00 & 0.00 \\
\hline
\end{tabular}




\begin{tabular}{|c|c|c|c|c|c|}
\hline AGAP4 & 1565620_PM_at & ArfGAP with GTPase domain, ankyrin repea & -1.2 & 0.00 & 0.00 \\
\hline NOP14-AS1 & 214123_PM_s_at & NOP14 antisense RNA 1 (non-protein codin! & -1.2 & 0.00 & 0.00 \\
\hline USP36 & 227093_PM_at & Ubiquitin specific peptidase 36 & -1.2 & 0.00 & 0.00 \\
\hline SLC38A10 & 1563226_PM_at & solute carrier family 38 , member 10 & -1.2 & 0.00 & 0.00 \\
\hline TNRC6A & 243834_PM_at & trinucleotide repeat containing $6 \mathrm{~A}$ & -1.2 & 0.00 & 0.00 \\
\hline TMEM198B & 227106_PM_at & transmembrane protein 198B, pseudogene & -1.2 & 0.00 & 0.00 \\
\hline--- & 230651_PM_at & --- & -1.2 & 0.00 & 0.00 \\
\hline ZNF330 & 209814_PM_at & zinc finger protein 330 & -1.2 & 0.00 & 0.00 \\
\hline--- & 238709_PM_at & --- & -1.2 & 0.00 & 0.00 \\
\hline P2RY10 & 1553856_PM_s_at & purinergic receptor P2Y, G-protein coupled, & -1.2 & 0.00 & 0.00 \\
\hline BCL11B & 222895_PM_s_at & B-cell CLL & -1.2 & 0.00 & 0.00 \\
\hline CAND1 & 243910_PM_x_at & cullin-associated and neddylation-dissociat & -1.2 & 0.00 & 0.00 \\
\hline--- & 238271_PM_x_at & --- & -1.2 & 0.00 & 0.00 \\
\hline--- & 1563109_PM_at & --- & -1.2 & 0.00 & 0.00 \\
\hline RORA & 241760_PM_x_at & RAR-related orphan receptor $A$ & -1.2 & 0.00 & 0.00 \\
\hline TRPM7 & 1565887_PM_at & Transient receptor potential cation channel & -1.2 & 0.00 & 0.00 \\
\hline--- & 1568866_PM_at & --- & -1.2 & 0.00 & 0.00 \\
\hline PTPLAD1 & 222404_PM_x_at & protein tyrosine phosphatase-like A domain & -1.2 & 0.00 & 0.00 \\
\hline--- & 241297_PM_at & --- & -1.2 & 0.00 & 0.00 \\
\hline--- & 238000_PM_at & --- & -1.2 & 0.00 & 0.00 \\
\hline--- & 240621_PM_at & --- & -1.2 & 0.00 & 0.00 \\
\hline--- & 242110_PM_at & --- & -1.2 & 0.00 & 0.00 \\
\hline TRAF5 & 204352_PM_at & TNF receptor-associated factor 5 & -1.2 & 0.00 & 0.00 \\
\hline TAF15 & 234168_PM_at & TAF15 RNA polymerase II, TATA box binding & -1.2 & 0.00 & 0.00 \\
\hline SLC16A7 & 210807_PM_s_at & solute carrier family 16 , member 7 (monocí & -1.2 & 0.00 & 0.00 \\
\hline--- & 1559101_PM_at & --- & -1.2 & 0.00 & 0.00 \\
\hline MMD & 244523_PM_at & monocyte to macrophage differentiation-as & -1.2 & 0.00 & 0.00 \\
\hline DKC1 & 216212_PM_s_at & dyskeratosis congenita 1 , dyskerin & -1.2 & 0.00 & 0.00 \\
\hline--- & 241932_PM_at & --- & -1.2 & 0.00 & 0.00 \\
\hline--- & 231222_PM_at & --- & -1.2 & 0.00 & 0.00 \\
\hline PPM1K & 235061_PM_at & protein phosphatase, $\mathrm{Mg} 2+$ & -1.2 & 0.00 & 0.00 \\
\hline--- & 242501_PM_at & --- & -1.2 & 0.00 & 0.00 \\
\hline MCF2L & 212935_PM_at & MCF.2 cell line derived transforming sequer & -1.2 & 0.00 & 0.00 \\
\hline
\end{tabular}




\begin{tabular}{|c|c|c|c|c|c|}
\hline NUCB2 & 229838_PM_at & nucleobindin 2 & -1.2 & 0.00 & 0.00 \\
\hline--- & 240050_PM_s_at & --- & -1.2 & 0.00 & 0.00 \\
\hline--- & 1563473_PM_at & --- & -1.2 & 0.00 & 0.00 \\
\hline PTPRS & 226571_PM_s_at & protein tyrosine phosphatase, receptor typ & -1.2 & 0.00 & 0.00 \\
\hline--- & 240174_PM_at & --- & -1.2 & 0.00 & 0.00 \\
\hline ERAP2 & 219759_PM_at & endoplasmic reticulum aminopeptidase 2 & -1.2 & 0.00 & 0.00 \\
\hline PML & 235508_PM_at & promyelocytic leukemia & -1.2 & 0.00 & 0.00 \\
\hline ADAM28 & 208268_PM_at & ADAM metallopeptidase domain 28 & -1.2 & 0.00 & 0.00 \\
\hline THEM4 & 243492_PM_at & thioesterase superfamily member 4 & -1.2 & 0.00 & 0.00 \\
\hline--- & 236346_PM_at & --- & -1.2 & 0.00 & 0.01 \\
\hline EIF3B & 242550_PM_at & eukaryotic translation initiation factor 3 , sul & -1.2 & 0.00 & 0.01 \\
\hline LMO7 & 242722_PM_at & LIM domain 7 & -1.2 & 0.00 & 0.01 \\
\hline NLRP7 & 237461_PM_at & NLR family, pyrin domain containing 7 & -1.2 & 0.00 & 0.01 \\
\hline P2RY10 & 214615_PM_at & purinergic receptor P2Y, G-protein coupled, & -1.2 & 0.00 & 0.01 \\
\hline SEL1L3 & 212311_PM_at & sel-1 suppressor of lin-12-like 3 (C. elegans) & -1.2 & 0.00 & 0.01 \\
\hline ZNF418 & 243439_PM_at & zinc finger protein 418 & -1.2 & 0.00 & 0.01 \\
\hline SNX19 & 1554986_PM_a_at & sorting nexin 19 & -1.2 & 0.00 & 0.01 \\
\hline--- & 223951_PM_at & --- & -1.2 & 0.00 & 0.01 \\
\hline--- & 237194_PM_at & --- & -1.2 & 0.00 & 0.01 \\
\hline IGK@ & 224795_PM_x_at & immunoglobulin kappa locus & -1.2 & 0.00 & 0.01 \\
\hline CCDC50 & 236831_PM_at & coiled-coil domain containing 50 & -1.2 & 0.00 & 0.01 \\
\hline--- & 1556769_PM_a_at & --- & -1.2 & 0.00 & 0.01 \\
\hline MPPED2 & 205413_PM_at & metallophosphoesterase domain containin & -1.2 & 0.00 & 0.01 \\
\hline ZFP82 & 1555793_PM_a_at & zinc finger protein 82 homolog (mouse) & -1.2 & 0.00 & 0.01 \\
\hline--- & 236427_PM_at & --- & -1.2 & 0.00 & 0.01 \\
\hline LOC100506965 & 226587_PM_at & uncharacterized LOC100506965 & -1.2 & 0.00 & 0.01 \\
\hline--- & 217482_PM_at & --- & -1.2 & 0.00 & 0.01 \\
\hline TASP1 & 219443_PM_at & taspase, threonine aspartase, 1 & -1.2 & 0.00 & 0.01 \\
\hline DPP4 & 203717_PM_at & dipeptidyl-peptidase 4 & -1.2 & 0.00 & 0.01 \\
\hline--- & 243257_PM_at & --- & -1.2 & 0.00 & 0.01 \\
\hline TEX10 & 218104_PM_at & testis expressed 10 & -1.19 & 0.00 & 0.00 \\
\hline LUC7L & 223546_PM_x_at & LUC7-like (S. cerevisiae) & -1.19 & 0.00 & 0.00 \\
\hline ZNF747 & 238606_PM_at & zinc finger protein 747 & -1.19 & 0.00 & 0.00 \\
\hline
\end{tabular}




\begin{tabular}{|c|c|c|c|c|c|}
\hline DENR & 238982_PM_at & density-regulated protein & -1.19 & 0.00 & 0.00 \\
\hline ZDHHC14 & 219247_PM_s_at & zinc finger, DHHC-type containing 14 & -1.19 & 0.00 & 0.00 \\
\hline TSR2 & 213079_PM_at & TSR2, 20S rRNA accumulation, homolog (S. & -1.19 & 0.00 & 0.00 \\
\hline РCBP2 & 229467_PM_at & poly $(\mathrm{rC})$ binding protein 2 & -1.19 & 0.00 & 0.00 \\
\hline--- & 1556818_PM_at & --- & -1.19 & 0.00 & 0.00 \\
\hline FOXK1 & 226715_PM_at & forkhead box K1 & -1.19 & 0.00 & 0.00 \\
\hline--- & 238651_PM_at & --- & -1.19 & 0.00 & 0.00 \\
\hline PCCB & 1557502_PM_at & Propionyl CoA carboxylase, beta polypeptid & -1.19 & 0.00 & 0.00 \\
\hline UGP2 & 232180_PM_at & UDP-glucose pyrophosphorylase 2 & -1.19 & 0.00 & 0.00 \\
\hline NOL9 & 218754_PM_at & nucleolar protein 9 & -1.19 & 0.00 & 0.00 \\
\hline SPG7 & 230885_PM_at & spastic paraplegia 7 (pure and complicated & -1.19 & 0.00 & 0.00 \\
\hline UNG & 202330_PM_s_at & uracil-DNA glycosylase & -1.19 & 0.00 & 0.00 \\
\hline--- & 243675_PM_at & --- & -1.19 & 0.00 & 0.00 \\
\hline ZFAND6 & 239757_PM_at & Zinc finger, AN1-type domain 6 & -1.19 & 0.00 & 0.00 \\
\hline CCDC88C & 231288_PM_at & coiled-coil domain containing $88 \mathrm{C}$ & -1.19 & 0.00 & 0.00 \\
\hline SLC9B2 & 229491_PM_at & solute carrier family 9 , subfamily $\mathrm{B}$ ( $\mathrm{NHA} 2, \mathrm{c}$ & -1.19 & 0.00 & 0.00 \\
\hline SHQ1 & 63009_PM_at & SHQ1 homolog (S. cerevisiae) & -1.19 & 0.00 & 0.00 \\
\hline--- & 239943_PM_x_at & --- & -1.19 & 0.00 & 0.00 \\
\hline HNRNPD & 209330_PM_s_at & heterogeneous nuclear ribonucleoprotein [ & -1.19 & 0.00 & 0.00 \\
\hline--- & 242337_PM_at & --- & -1.19 & 0.00 & 0.00 \\
\hline CEP104 & 243696_PM_at & centrosomal protein $104 \mathrm{kDa}$ & -1.19 & 0.00 & 0.00 \\
\hline WHSC1 & 209053_PM_s_at & Wolf-Hirschhorn syndrome candidate 1 & -1.19 & 0.00 & 0.00 \\
\hline--- & 244711_PM_at & --- & -1.19 & 0.00 & 0.00 \\
\hline 6-Sep & 213666_PM_at & septin 6 & -1.19 & 0.00 & 0.00 \\
\hline HERC2 & 217902_PM_s_at & HECT and RLD domain containing E3 ubiqui 1 & -1.19 & 0.00 & 0.00 \\
\hline CDC14A & 210743_PM_s_at & CDC14 cell division cycle 14 homolog A (S. C & -1.19 & 0.00 & 0.00 \\
\hline--- & 236237_PM_at & --- & -1.19 & 0.00 & 0.00 \\
\hline TNFRSF25 & 211841_PM_s_at & tumor necrosis factor receptor superfamily, & -1.19 & 0.00 & 0.00 \\
\hline USP40 & 225089_PM_at & ubiquitin specific peptidase 40 & -1.19 & 0.00 & 0.00 \\
\hline TSPY26P & 236369_PM_at & testis specific protein, Y-linked 26, pseudog & -1.19 & 0.00 & 0.00 \\
\hline TMEM63A & 215583_PM_at & Transmembrane protein $63 \mathrm{~A}$ & -1.19 & 0.00 & 0.00 \\
\hline MCM3AP-AS1 & 232740_PM_at & MCM3AP antisense RNA 1 (non-protein cod & -1.19 & 0.00 & 0.00 \\
\hline C21orf2 & 203996_PM_s_at & chromosome 21 open reading frame 2 & -1.19 & 0.00 & 0.00 \\
\hline
\end{tabular}




\begin{tabular}{|c|c|c|c|c|c|}
\hline C21orf2 & 203995_PM_at & chromosome 21 open reading frame 2 & -1.19 & 0.00 & 0.00 \\
\hline--- & 237383_PM_at & --- & -1.19 & 0.00 & 0.00 \\
\hline BCCIP & 218264_PM_at & BRCA2 and CDKN1A interacting protein & -1.19 & 0.00 & 0.00 \\
\hline--- & 238040_PM_at & --- & -1.19 & 0.00 & 0.00 \\
\hline--- & 239110_PM_s_at & --- & -1.19 & 0.00 & 0.00 \\
\hline CXCR7 & 232746_PM_at & Chemokine (C-X-C motif) receptor 7 & -1.19 & 0.00 & 0.00 \\
\hline ZNF862 & 226808_PM_at & zinc finger protein 862 & -1.19 & 0.00 & 0.00 \\
\hline--- & 231329_PM_at & --- & -1.19 & 0.00 & 0.00 \\
\hline--- & 1559437_PM_at & --- & -1.19 & 0.00 & 0.00 \\
\hline--- & 1564733_PM_at & --- & -1.19 & 0.00 & 0.00 \\
\hline--- & 215376_PM_at & --- & -1.19 & 0.00 & 0.00 \\
\hline ITPKB & 235213_PM_at & Inositol-trisphosphate 3-kinase B & -1.19 & 0.00 & 0.00 \\
\hline CHD6 & 1555463_PM_a_at & chromodomain helicase DNA binding protei & -1.19 & 0.00 & 0.00 \\
\hline MAP4K4 & 1558732_PM_at & mitogen-activated protein kinase kinase kin & -1.19 & 0.00 & 0.00 \\
\hline--- & 1568867_PM_x_at & --- & -1.19 & 0.00 & 0.00 \\
\hline BCOR & 223915_PM_at & BCL6 corepressor & -1.19 & 0.00 & 0.00 \\
\hline COPG2IT1 & 213486_PM_at & COPG2 imprinted transcript 1 (non-protein & -1.19 & 0.00 & 0.00 \\
\hline--- & 1560082_PM_at & --- & -1.19 & 0.00 & 0.00 \\
\hline WDR4 & 241937_PM_s_at & WD repeat domain 4 & -1.19 & 0.00 & 0.00 \\
\hline LOC100131564 & 227074_PM_at & uncharacterized LOC100131564 & -1.19 & 0.00 & 0.00 \\
\hline ZNHIT6 & 233005_PM_at & zinc finger, HIT-type containing 6 & -1.19 & 0.00 & 0.00 \\
\hline ECHDC2 & 235305_PM_s_at & enoyl CoA hydratase domain containing 2 & -1.19 & 0.00 & 0.00 \\
\hline GEMIN8 & 219252_PM_s_at & gem (nuclear organelle) associated protein & -1.19 & 0.00 & 0.00 \\
\hline KIAA0754 & 215268_PM_at & KIAA0754 & -1.19 & 0.00 & 0.00 \\
\hline $\mathrm{BCCIP}$ & 227322_PM_s_at & BRCA2 and CDKN1A interacting protein & -1.19 & 0.00 & 0.00 \\
\hline--- & 244408_PM_at & --- & -1.19 & 0.00 & 0.00 \\
\hline--- & 220612_PM_at & --- & -1.19 & 0.00 & 0.00 \\
\hline--- & 239167_PM_at & --- & -1.19 & 0.00 & 0.00 \\
\hline--- & 237001_PM_at & --- & -1.19 & 0.00 & 0.00 \\
\hline--- & 239646_PM_at & --- & -1.19 & 0.00 & 0.00 \\
\hline C1orf186 & 222281_PM_s_at & chromosome 1 open reading frame 186 & -1.19 & 0.00 & 0.00 \\
\hline --- & 239978_PM_at & --- & -1.19 & 0.00 & 0.00 \\
\hline--- & 244341_PM_at & --- & -1.19 & 0.00 & 0.00 \\
\hline
\end{tabular}




\begin{tabular}{|c|c|c|c|c|c|}
\hline SLC1A4 & 244377_PM_at & Solute carrier family 1 (glutamate & -1.19 & 0.00 & 0.00 \\
\hline--- & 1563553_PM_at & --- & -1.19 & 0.00 & 0.00 \\
\hline $\mathrm{ZCCHC7}$ & 230332_PM_at & Zinc finger, $\mathrm{CCHC}$ domain containing 7 & -1.19 & 0.00 & 0.00 \\
\hline--- & 238938_PM_at & --- & -1.19 & 0.00 & 0.00 \\
\hline MAGEE1 & 1556047_PM_s_at & melanoma antigen family $E, 1$ & -1.19 & 0.00 & 0.00 \\
\hline DOCK10 & 215151_PM_at & dedicator of cytokinesis 10 & -1.19 & 0.00 & 0.00 \\
\hline IRS1 & 238933_PM_at & insulin receptor substrate 1 & -1.19 & 0.00 & 0.00 \\
\hline HSF2 & 209657_PM_s_at & heat shock transcription factor 2 & -1.19 & 0.00 & 0.00 \\
\hline--- & 229858_PM_at & --- & -1.19 & 0.00 & 0.00 \\
\hline SRRM2 & 208610_PM_s_at & serine & -1.19 & 0.00 & 0.00 \\
\hline--- & 243003_PM_at & --- & -1.19 & 0.00 & 0.00 \\
\hline RAB14 & 200928_PM_s_at & RAB14, member RAS oncogene family & -1.19 & 0.00 & 0.00 \\
\hline ZNF512B & 55872_PM_at & zinc finger protein $512 \mathrm{~B}$ & -1.19 & 0.00 & 0.00 \\
\hline--- & 237803_PM_x_at & --- & -1.19 & 0.00 & 0.00 \\
\hline--- & 244473_PM_at & --- & -1.19 & 0.00 & 0.00 \\
\hline--- & 238558_PM_at & --- & -1.19 & 0.00 & 0.01 \\
\hline NPR2 & 204310_PM_s_at & natriuretic peptide receptor $B$ & -1.19 & 0.00 & 0.01 \\
\hline LOC100506776 & 239237_PM_at & uncharacterized LOC100506776 & -1.19 & 0.00 & 0.01 \\
\hline INTS2 & 224308_PM_s_at & integrator complex subunit 2 & -1.19 & 0.00 & 0.01 \\
\hline TRAF3IP1 & 238494_PM_at & TNF receptor-associated factor 3 interacting & -1.19 & 0.00 & 0.01 \\
\hline LARP1B & 226750_PM_at & La ribonucleoprotein domain family, memb & -1.19 & 0.00 & 0.01 \\
\hline--- & 230233_PM_at & --- & -1.19 & 0.00 & 0.01 \\
\hline--- & 242270_PM_at & --- & -1.19 & 0.00 & 0.01 \\
\hline KIAA1919 & 242851_PM_at & KIAA1919 & -1.19 & 0.00 & 0.01 \\
\hline NMNAT3 & 228090_PM_at & nicotinamide nucleotide adenylyltransferas & -1.19 & 0.00 & 0.01 \\
\hline--- & 239995_PM_at & --- & -1.19 & 0.00 & 0.01 \\
\hline ZNF831 & 1558827_PM_a_at & zinc finger protein 831 & -1.19 & 0.00 & 0.01 \\
\hline PNOC & 205901_PM_at & prepronociceptin & -1.19 & 0.00 & 0.01 \\
\hline--- & 232333_PM_at & --- & -1.19 & 0.00 & 0.01 \\
\hline--- & 1564798_PM_at & --- & -1.19 & 0.00 & 0.01 \\
\hline LOC100506029 & 228661_PM_s_at & uncharacterized LOC100506029 & -1.19 & 0.00 & 0.01 \\
\hline --- & 241823_PM_at & --- & -1.19 & 0.00 & 0.01 \\
\hline--- & 215577_PM_at & --- & -1.19 & 0.00 & 0.01 \\
\hline
\end{tabular}




\begin{tabular}{|c|c|c|c|c|c|}
\hline QSER1 & 229982_PM_at & glutamine and serine rich 1 & -1.19 & 0.00 & 0.01 \\
\hline--- & 233713_PM_at & --- & -1.19 & 0.00 & 0.01 \\
\hline AKAP2 & 202759_PM_s_at & A kinase (PRKA) anchor protein 2 & -1.19 & 0.00 & 0.01 \\
\hline--- & 217322_PM_x_at & --- & -1.19 & 0.00 & 0.01 \\
\hline--- & 232795_PM_at & --- & -1.19 & 0.00 & 0.01 \\
\hline LAMC1 & 200771_PM_at & laminin, gamma 1 (formerly LAMB2) & -1.19 & 0.00 & 0.01 \\
\hline MYEF2 & 222771_PM_s_at & myelin expression factor 2 & -1.19 & 0.00 & 0.01 \\
\hline CYB561 & 209164_PM_s_at & cytochrome b-561 & -1.19 & 0.00 & 0.01 \\
\hline NMT2 & 215743_PM_at & N-myristoyltransferase 2 & -1.19 & 0.00 & 0.02 \\
\hline RBM4 & 213718_PM_at & RNA binding motif protein 4 & -1.18 & 0.00 & 0.00 \\
\hline DIS3L2 & 238602_PM_at & DIS3 mitotic control homolog (S. cerevisiae) & -1.18 & 0.00 & 0.00 \\
\hline DNAJC16 & 212908_PM_at & DnaJ (Hsp40) homolog, subfamily C, membe & -1.18 & 0.00 & 0.00 \\
\hline TEX10 & 1558702_PM_at & testis expressed 10 & -1.18 & 0.00 & 0.00 \\
\hline SYNPO & 202796_PM_at & synaptopodin & -1.18 & 0.00 & 0.00 \\
\hline--- & 242725_PM_at & --- & -1.18 & 0.00 & 0.00 \\
\hline--- & 239469_PM_at & --- & -1.18 & 0.00 & 0.00 \\
\hline CSTF3 & 229666_PM_s_at & cleavage stimulation factor, 3' pre-RNA, suk & -1.18 & 0.00 & 0.00 \\
\hline GPM6B & 209169_PM_at & glycoprotein M6B & -1.18 & 0.00 & 0.00 \\
\hline SHQ1 & 219083_PM_at & SHQ1 homolog (S. cerevisiae) & -1.18 & 0.00 & 0.00 \\
\hline NEK1 & 213328_PM_at & NIMA (never in mitosis gene a)-related kina & -1.18 & 0.00 & 0.00 \\
\hline--- & 235434_PM_at & --- & -1.18 & 0.00 & 0.00 \\
\hline--- & 236607_PM_at & --- & -1.18 & 0.00 & 0.00 \\
\hline METTL17 & 223528_PM_s_at & methyltransferase like 17 & -1.18 & 0.00 & 0.00 \\
\hline ZSCAN2 & 231188_PM_at & zinc finger and SCAN domain containing 2 & -1.18 & 0.00 & 0.00 \\
\hline YME1L1 & 224343_PM_x_at & YME1-like 1 (S. cerevisiae) & -1.18 & 0.00 & 0.00 \\
\hline--- & 239679_PM_at & --- & -1.18 & 0.00 & 0.00 \\
\hline EXOSC7 & 213648_PM_at & exosome component 7 & -1.18 & 0.00 & 0.00 \\
\hline CSPP1 & 220072_PM_at & centrosome and spindle pole associated prc & -1.18 & 0.00 & 0.00 \\
\hline IL12RB2 & 206999_PM_at & interleukin 12 receptor, beta 2 & -1.18 & 0.00 & 0.00 \\
\hline ZNF248 & 213269_PM_at & zinc finger protein 248 & -1.18 & 0.00 & 0.00 \\
\hline KDSR & 202419_PM_at & 3-ketodihydrosphingosine reductase & -1.18 & 0.00 & 0.00 \\
\hline NHLRC3 & 236953_PM_s_at & NHL repeat containing 3 & -1.18 & 0.00 & 0.00 \\
\hline ZNF830 & 226315_PM_at & zinc finger protein 830 & -1.18 & 0.00 & 0.00 \\
\hline
\end{tabular}




\begin{tabular}{|c|c|c|c|c|}
\hline COBLL1 & 215393_PM_s_at & COBL-like 1 & -1.18 & 0.00 \\
\hline--- & 234507_PM_at & --- & -1.18 & 0.00 \\
\hline LOC100506469 & 222280_PM_at & uncharacterized LOC100506469 & -1.18 & 0.00 \\
\hline--- & 235601_PM_at & --- & -1.18 & 0.00 \\
\hline ZNHIT6 & 218932_PM_at & zinc finger, HIT-type containing 6 & -1.18 & 0.00 \\
\hline SLC7A6 & 203580_PM_s_at & solute carrier family 7 (amino acid transpor & -1.18 & 0.00 \\
\hline--- & 1557539_PM_at & --- & -1.18 & 0.00 \\
\hline--- & 239091_PM_at & --- & -1.18 & 0.00 \\
\hline LOC100506694 & 235322_PM_at & uncharacterized LOC100506694 & -1.18 & 0.00 \\
\hline WHAMMP2 & 222367_PM_at & WAS protein homolog associated with actin & -1.18 & 0.00 \\
\hline RPS15A & 235309_PM_at & ribosomal protein S15a & -1.18 & 0.00 \\
\hline LOC100422781 & 1561304_PM_a_at & uncharacterized LOC100422781 & -1.18 & 0.00 \\
\hline--- & 243303_PM_at & --- & -1.18 & 0.00 \\
\hline PTPN2 & 241623_PM_at & Protein tyrosine phosphatase, non-receptol & -1.18 & 0.00 \\
\hline TMEM194A & 212621_PM_at & transmembrane protein $194 \mathrm{~A}$ & -1.18 & 0.00 \\
\hline ENGASE & 65635_PM_at & endo-beta- $\mathrm{N}$-acetylglucosaminidase & -1.18 & 0.00 \\
\hline HHAT & 219687_PM_at & hedgehog acyltransferase & -1.18 & 0.00 \\
\hline GTPBP4 & 218239_PM_s_at & GTP binding protein 4 & -1.18 & 0.00 \\
\hline MPHOSPH9 & 1558369_PM_at & M-phase phosphoprotein 9 & -1.18 & 0.00 \\
\hline--- & 232583_PM_at & --- & -1.18 & 0.00 \\
\hline TSPYL2 & 218012_PM_at & TSPY-like 2 & -1.18 & 0.00 \\
\hline DNAJC30 & 239018_PM_at & DnaJ (Hsp40) homolog, subfamily C, membє & -1.18 & 0.00 \\
\hline NAP1L4 & 1556567_PM_at & nucleosome assembly protein 1-like 4 & -1.18 & 0.00 \\
\hline PIK3IP1 & 221757_PM_at & phosphoinositide-3-kinase interacting prot $\epsilon$ & -1.18 & 0.00 \\
\hline CHD6 & 225026_PM_at & chromodomain helicase DNA binding protei & -1.18 & 0.00 \\
\hline--- & 1557263_PM_s_at & --- & -1.18 & 0.00 \\
\hline SNHG12 & 228990_PM_at & small nucleolar RNA host gene 12 (non-prot & -1.18 & 0.00 \\
\hline USP36 & 220370_PM_s_at & ubiquitin specific peptidase 36 & -1.18 & 0.00 \\
\hline RGP1 & 203169_PM_at & RGP1 retrograde golgi transport homolog ( $₫$ & -1.18 & 0.00 \\
\hline FBXO21 & 212229_PM_s_at & F-box protein 21 & -1.18 & 0.00 \\
\hline LOC283174 & 232123_PM_at & uncharacterized LOC283174 & -1.18 & 0.00 \\
\hline $\mathrm{C} 2 \mathrm{CD} 2$ & 212875_PM_s_at & C2 calcium-dependent domain containing 2 & -1.18 & 0.00 \\
\hline HAUS5 & 36888_PM_at & HAUS augmin-like complex, subunit 5 & -1.18 & 0.00 \\
\hline
\end{tabular}




\begin{tabular}{|c|c|c|c|c|c|}
\hline WRN & 205667_PM_at & Werner syndrome, RecQ helicase-like & -1.18 & 0.00 & 0.00 \\
\hline MRPS25 & 226848_PM_at & mitochondrial ribosomal protein S25 & -1.18 & 0.00 & 0.00 \\
\hline--- & 233678_PM_at & --- & -1.18 & 0.00 & 0.00 \\
\hline RAPH1 & 231075_PM_x_at & Ras association (RalGDS & -1.18 & 0.00 & 0.00 \\
\hline MIR1204 & 222087_PM_at & microRNA 1204 & -1.18 & 0.00 & 0.00 \\
\hline--- & 215140_PM_at & --- & -1.18 & 0.00 & 0.00 \\
\hline--- & 1570166_PM_a_at & --- & -1.18 & 0.00 & 0.00 \\
\hline DDHD2 & 212690_PM_at & DDHD domain containing 2 & -1.18 & 0.00 & 0.00 \\
\hline FMNL3 & 232249_PM_at & formin-like 3 & -1.18 & 0.00 & 0.00 \\
\hline LRRC69 & 238214_PM_at & leucine rich repeat containing 69 & -1.18 & 0.00 & 0.00 \\
\hline--- & 243154_PM_at & --- & -1.18 & 0.00 & 0.00 \\
\hline ENGASE & 220349_PM_s_at & endo-beta- $\mathrm{N}$-acetylglucosaminidase & -1.18 & 0.00 & 0.00 \\
\hline--- & 242068_PM_at & --- & -1.18 & 0.00 & 0.00 \\
\hline--- & 231259_PM_s_at & --- & -1.18 & 0.00 & 0.00 \\
\hline $\mathrm{BICD} 1$ & 214806_PM_at & bicaudal D homolog 1 (Drosophila) & -1.18 & 0.00 & 0.00 \\
\hline TXLNG & 219969_PM_at & taxilin gamma & -1.18 & 0.00 & 0.00 \\
\hline--- & 241845_PM_at & --- & -1.18 & 0.00 & 0.00 \\
\hline BCAS4 & 228787_PM_s_at & breast carcinoma amplified sequence 4 & -1.18 & 0.00 & 0.00 \\
\hline--- & 1558922_PM_at & --- & -1.18 & 0.00 & 0.00 \\
\hline--- & 232779_PM_at & --- & -1.18 & 0.00 & 0.00 \\
\hline RNASEH2B & 215040_PM_at & Ribonuclease $\mathrm{H} 2$, subunit $\mathrm{B}$ & -1.18 & 0.00 & 0.00 \\
\hline--- & 242983_PM_at & --- & -1.18 & 0.00 & 0.00 \\
\hline--- & 232236_PM_at & --- & -1.18 & 0.00 & 0.00 \\
\hline SLC38A10 & 1563228_PM_x_at & solute carrier family 38 , member 10 & -1.18 & 0.00 & 0.00 \\
\hline GGA2 & 213772_PM_s_at & golgi-associated, gamma adaptin ear contai & -1.18 & 0.00 & 0.00 \\
\hline--- & 244606_PM_at & --- & -1.18 & 0.00 & 0.00 \\
\hline SRR & 222844_PM_s_at & serine racemase & -1.18 & 0.00 & 0.00 \\
\hline--- & 240007_PM_at & --- & -1.18 & 0.00 & 0.00 \\
\hline RAD51C & 209849_PM_s_at & RAD51 homolog C (S. cerevisiae) & -1.18 & 0.00 & 0.00 \\
\hline--- & 237441_PM_at & --- & -1.18 & 0.00 & 0.00 \\
\hline TCERG1 & 229706_PM_at & transcription elongation regulator 1 & -1.18 & 0.00 & 0.00 \\
\hline AURKAPS1 & 216057_PM_at & aurora kinase $\mathrm{A}$ pseudogene 1 & -1.18 & 0.00 & 0.00 \\
\hline NXT1 & 218708_PM_at & NTF2-like export factor 1 & -1.18 & 0.00 & 0.00 \\
\hline
\end{tabular}




\begin{tabular}{|c|c|c|c|c|c|}
\hline WDR67 & 214061_PM_at & WD repeat domain 67 & -1.18 & 0.00 & 0.00 \\
\hline--- & 231316_PM_at & --- & -1.18 & 0.00 & 0.00 \\
\hline--- & 1562412_PM_at & --- & -1.18 & 0.00 & 0.00 \\
\hline--- & 230415_PM_at & --- & -1.18 & 0.00 & 0.00 \\
\hline NME9 & 1562619_PM_at & NME & -1.18 & 0.00 & 0.00 \\
\hline--- & 243467_PM_at & --- & -1.18 & 0.00 & 0.00 \\
\hline LOC96610 & 233132_PM_at & BMS1 homolog, ribosome assembly protein & -1.18 & 0.00 & 0.00 \\
\hline OXNAD1 & 227686_PM_at & oxidoreductase NAD-binding domain contai & -1.18 & 0.00 & 0.00 \\
\hline--- & 232447_PM_at & --- & -1.18 & 0.00 & 0.00 \\
\hline TMEM14A & 218477_PM_at & transmembrane protein $14 \mathrm{~A}$ & -1.18 & 0.00 & 0.01 \\
\hline--- & 243626_PM_at & --- & -1.18 & 0.00 & 0.01 \\
\hline RUNX1-IT1 & 220918_PM_at & RUNX1 intronic transcript 1 (non-protein co & -1.18 & 0.00 & 0.01 \\
\hline PGM5-AS1 & 231024_PM_at & PGM5 antisense RNA 1 (non-protein coding & -1.18 & 0.00 & 0.01 \\
\hline RAPH1 & 225189_PM_s_at & Ras association (RalGDS & -1.18 & 0.00 & 0.01 \\
\hline--- & 242890_PM_at & --- & -1.18 & 0.00 & 0.01 \\
\hline SEL1L3 & 235353_PM_at & sel-1 suppressor of lin-12-like 3 (C. elegans) & -1.18 & 0.00 & 0.01 \\
\hline sox6 & 235526_PM_at & SRY (sex determining region Y)-box 6 & -1.18 & 0.00 & 0.01 \\
\hline CRTC3 & 232879_PM_at & CREB regulated transcription coactivator 3 & -1.18 & 0.00 & 0.01 \\
\hline--- & 231351_PM_at & --- & -1.18 & 0.00 & 0.01 \\
\hline HSP90AB1 & 214359_PM_s_at & heat shock protein 90kDa alpha (cytosolic), & -1.18 & 0.00 & 0.01 \\
\hline IGHV5-78 & 210450_PM_at & immunoglobulin heavy variable 5-78 (pseuc & -1.18 & 0.00 & 0.01 \\
\hline--- & 235920_PM_at & --- & -1.18 & 0.00 & 0.01 \\
\hline--- & 216278_PM_at & --- & -1.18 & 0.00 & 0.01 \\
\hline MTMR2 & 203212_PM_s_at & myotubularin related protein 2 & -1.18 & 0.00 & 0.01 \\
\hline--- & 1558801_PM_at & --- & -1.18 & 0.00 & 0.01 \\
\hline RLTPR & 227216_PM_at & RGD motif, leucine rich repeats, tropomodu & -1.18 & 0.00 & 0.01 \\
\hline KIAA0368 & 236368_PM_at & KIAA0368 & -1.18 & 0.00 & 0.01 \\
\hline AAK1 & 214956_PM_at & AP2 associated kinase 1 & -1.18 & 0.00 & 0.01 \\
\hline TCF7 & 205254_PM_x_at & transcription factor 7 (T-cell specific, HMG-l & -1.18 & 0.00 & 0.01 \\
\hline--- & 216000_PM_at & --- & -1.18 & 0.00 & 0.01 \\
\hline PPAPDC1B & 223569_PM_at & phosphatidic acid phosphatase type 2 dom & -1.18 & 0.00 & 0.01 \\
\hline RHPN2 & 227196_PM_at & rhophilin, Rho GTPase binding protein 2 & -1.18 & 0.00 & 0.01 \\
\hline--- & 229293_PM_at & --- & -1.18 & 0.00 & 0.01 \\
\hline
\end{tabular}




\begin{tabular}{|c|c|c|c|c|c|}
\hline NAPEPLD & 242635_PM_s_at & $\mathrm{N}$-acyl phosphatidylethanolamine phosphol & -1.18 & 0.00 & 0.01 \\
\hline HSP90AB1 & 1557910_PM_at & heat shock protein 90kDa alpha (cytosolic), & -1.18 & 0.00 & 0.01 \\
\hline--- & 1566709_PM_at & --- & -1.18 & 0.00 & 0.01 \\
\hline KANSL1 & 243589_PM_at & KAT8 regulatory NSL complex subunit 1 & -1.18 & 0.00 & 0.01 \\
\hline PPAPDC1B & 223568_PM_s_at & phosphatidic acid phosphatase type 2 dom & -1.18 & 0.00 & 0.01 \\
\hline--- & 232369_PM_at & --- & -1.18 & 0.00 & 0.01 \\
\hline FLJ10038 & 205511_PM_at & uncharacterized protein FLJ10038 & -1.18 & 0.00 & 0.01 \\
\hline FAM102A & 212400_PM_at & family with sequence similarity 102, memb & -1.18 & 0.00 & 0.01 \\
\hline--- & 228818_PM_at & --- & -1.18 & 0.00 & 0.01 \\
\hline SLC35F2 & 218826_PM_at & solute carrier family 35 , member F2 & -1.18 & 0.00 & 0.01 \\
\hline--- & 235925_PM_at & --- & -1.18 & 0.00 & 0.01 \\
\hline C1QTNF3 & 1561263_PM_at & $\mathrm{C} 1 \mathrm{q}$ and tumor necrosis factor related prot $\epsilon$ & -1.18 & 0.00 & 0.01 \\
\hline--- & 241936_PM_x_at & --- & -1.18 & 0.00 & 0.01 \\
\hline PEX5 & 203244_PM_at & peroxisomal biogenesis factor 5 & -1.18 & 0.00 & 0.01 \\
\hline--- & 234645_PM_at & --- & -1.18 & 0.00 & 0.01 \\
\hline--- & 241561_PM_at & --- & -1.18 & 0.00 & 0.01 \\
\hline CD1C & 205987_PM_at & CD1c molecule & -1.18 & 0.00 & 0.01 \\
\hline LRCH3 & 240926_PM_at & leucine-rich repeats and calponin homology & -1.18 & 0.00 & 0.01 \\
\hline--- & 240601_PM_at & --- & -1.18 & 0.00 & 0.01 \\
\hline ACSS1 & 234801_PM_s_at & acyl-CoA synthetase short-chain family mer & -1.18 & 0.00 & 0.01 \\
\hline SLC35E2 & 215169_PM_at & solute carrier family 35 , member E2 & -1.18 & 0.00 & 0.01 \\
\hline NASP & 201969_PM_at & nuclear autoantigenic sperm protein (histor & -1.17 & 0.00 & 0.00 \\
\hline TGIF2 & 216262_PM_s_at & TGFB-induced factor homeobox 2 & -1.17 & 0.00 & 0.00 \\
\hline LUC7L & 220143_PM_x_at & LUC7-like (S. cerevisiae) & -1.17 & 0.00 & 0.00 \\
\hline ZBTB44 & 220243_PM_at & zinc finger and BTB domain containing 44 & -1.17 & 0.00 & 0.00 \\
\hline FAM165B & 228239_PM_at & family with sequence similarity 165, memb & -1.17 & 0.00 & 0.00 \\
\hline PAPD7 & 202466_PM_at & PAP associated domain containing 7 & -1.17 & 0.00 & 0.00 \\
\hline--- & 234164_PM_at & --- & -1.17 & 0.00 & 0.00 \\
\hline--- & 244826_PM_at & --- & -1.17 & 0.00 & 0.00 \\
\hline--- & 242431_PM_at & --- & -1.17 & 0.00 & 0.00 \\
\hline RBFA & 219419_PM_at & ribosome binding factor $A$ (putative) & -1.17 & 0.00 & 0.00 \\
\hline --- & 243249_PM_at & --- & -1.17 & 0.00 & 0.00 \\
\hline SUPV3L1 & 212894_PM_at & suppressor of var1, 3-like 1 (S. cerevisiae) & -1.17 & 0.00 & 0.00 \\
\hline
\end{tabular}




\begin{tabular}{|c|c|c|c|c|c|}
\hline--- & 228156_PM_at & --- & -1.17 & 0.00 & 0.00 \\
\hline TRIT1 & 218617_PM_at & tRNA isopentenyltransferase 1 & -1.17 & 0.00 & 0.00 \\
\hline USP34 & 233595_PM_at & ubiquitin specific peptidase 34 & -1.17 & 0.00 & 0.00 \\
\hline TNRC6A & 234734_PM_s_at & trinucleotide repeat containing $6 \mathrm{~A}$ & -1.17 & 0.00 & 0.00 \\
\hline RAD52 & 205647_PM_at & RAD52 homolog (S. cerevisiae) & -1.17 & 0.00 & 0.00 \\
\hline--- & 240121_PM_x_at & --- & -1.17 & 0.00 & 0.00 \\
\hline NAPEPLD & 238722_PM_x_at & $\mathrm{N}$-acyl phosphatidylethanolamine phosphol & -1.17 & 0.00 & 0.00 \\
\hline RIMKLB & 229344_PM_x_at & ribosomal modification protein rimK-like fal & -1.17 & 0.00 & 0.00 \\
\hline METTL17 & 223988_PM_x_at & methyltransferase like 17 & -1.17 & 0.00 & 0.00 \\
\hline SFSWAP & 202773_PM_s_at & splicing factor, suppressor of white-apricot & -1.17 & 0.00 & 0.00 \\
\hline SHMT2 & 214096_PM_s_at & serine hydroxymethyltransferase 2 (mitoch & -1.17 & 0.00 & 0.00 \\
\hline--- & 236883_PM_at & --- & -1.17 & 0.00 & 0.00 \\
\hline PIK3C2A & 241905_PM_at & Phosphoinositide-3-kinase, class 2, alpha pc & -1.17 & 0.00 & 0.00 \\
\hline WDR3 & 218882_PM_s_at & WD repeat domain 3 & -1.17 & 0.00 & 0.00 \\
\hline--- & 1553547_PM_at & --- & -1.17 & 0.00 & 0.00 \\
\hline C3orf37 & 201677_PM_at & Chromosome 3 open reading frame 37 & -1.17 & 0.00 & 0.00 \\
\hline CNOT7 & 233019_PM_at & CCR4-NOT transcription complex, subunit 7 & -1.17 & 0.00 & 0.00 \\
\hline TPT1-AS1 & 228913_PM_at & TPT1 antisense RNA 1 (non-protein coding) & -1.17 & 0.00 & 0.00 \\
\hline MUM1 & 229033_PM_s_at & melanoma associated antigen (mutated) 1 & -1.17 & 0.00 & 0.00 \\
\hline MUM1 & 223348_PM_x_at & melanoma associated antigen (mutated) 1 & -1.17 & 0.00 & 0.00 \\
\hline PPARD & 37152_PM_at & peroxisome proliferator-activated receptor & -1.17 & 0.00 & 0.00 \\
\hline SLC38A9 & 243709_PM_at & solute carrier family 38 , member 9 & -1.17 & 0.00 & 0.00 \\
\hline REV3L & 238736_PM_at & REV3-like, catalytic subunit of DNA polymer & -1.17 & 0.00 & 0.00 \\
\hline TSPAN3 & 200973_PM_s_at & tetraspanin 3 & -1.17 & 0.00 & 0.00 \\
\hline ITPR1 & 211323_PM_s_at & inositol 1,4,5-trisphosphate receptor, type: & -1.17 & 0.00 & 0.00 \\
\hline --- & 242343_PM_x_at & --- & -1.17 & 0.00 & 0.00 \\
\hline PELP1 & 215354_PM_s_at & proline, glutamate and leucine rich protein & -1.17 & 0.00 & 0.00 \\
\hline MTERFD3 & 225341_PM_at & MTERF domain containing 3 & -1.17 & 0.00 & 0.00 \\
\hline--- & 242650_PM_at & --- & -1.17 & 0.00 & 0.00 \\
\hline C4orf29 & 219980_PM_at & chromosome 4 open reading frame 29 & -1.17 & 0.00 & 0.00 \\
\hline RBM5 & 230742_PM_at & RNA binding motif protein 5 & -1.17 & 0.00 & 0.00 \\
\hline--- & 243869_PM_at & --- & -1.17 & 0.00 & 0.00 \\
\hline--- & 243691_PM_at & --- & -1.17 & 0.00 & 0.00 \\
\hline
\end{tabular}




\begin{tabular}{|c|c|c|c|c|c|}
\hline KIAA0494 & 1559023_PM_a_at & KIAA0494 & -1.17 & 0.00 & 0.00 \\
\hline--- & 233161_PM_at & --- & -1.17 & 0.00 & 0.00 \\
\hline--- & 1570552_PM_at & --- & -1.17 & 0.00 & 0.00 \\
\hline--- & 232125_PM_at & --- & -1.17 & 0.00 & 0.00 \\
\hline--- & 241885_PM_at & --- & -1.17 & 0.00 & 0.00 \\
\hline KIAA0664L3 & 219442_PM_at & KIAA0664-like 3 & -1.17 & 0.00 & 0.00 \\
\hline CXXC5 & 222996_PM_s_at & CXXC finger protein 5 & -1.17 & 0.00 & 0.00 \\
\hline TMEM99 & 226565_PM_at & transmembrane protein 99 & -1.17 & 0.00 & 0.00 \\
\hline SNX9 & 223028_PM_s_at & sorting nexin 9 & -1.17 & 0.00 & 0.00 \\
\hline STMN1 & 200783_PM_s_at & stathmin 1 & -1.17 & 0.00 & 0.00 \\
\hline PPRC1 & 203737_PM_s_at & peroxisome proliferator-activated receptor & -1.17 & 0.00 & 0.00 \\
\hline SSB & 201138_PM_s_at & Sjogren syndrome antigen $B$ (autoantigen $\mathrm{L}_{i}$ & -1.17 & 0.00 & 0.00 \\
\hline--- & 215406_PM_at & --- & -1.17 & 0.00 & 0.00 \\
\hline FAM60A & 223038_PM_s_at & family with sequence similarity 60, member & -1.17 & 0.00 & 0.00 \\
\hline AGPAT4 & 219693_PM_at & 1-acylglycerol-3-phosphate O-acyltransfera: & -1.17 & 0.00 & 0.00 \\
\hline SYNJ2BP & 219156_PM_at & synaptojanin 2 binding protein & -1.17 & 0.00 & 0.00 \\
\hline CD6 & 211893_PM_x_at & CD6 molecule & -1.17 & 0.00 & 0.00 \\
\hline--- & 236751_PM_at & --- & -1.17 & 0.00 & 0.00 \\
\hline SPOCK2 & 202523_PM_s_at & sparc & -1.17 & 0.00 & 0.00 \\
\hline--- & 243300_PM_at & --- & -1.17 & 0.00 & 0.00 \\
\hline--- & 239540_PM_at & --- & -1.17 & 0.00 & 0.00 \\
\hline LEF1 & 221558_PM_s_at & lymphoid enhancer-binding factor 1 & -1.17 & 0.00 & 0.00 \\
\hline PDE6B & 238153_PM_at & phosphodiesterase 6B, cGMP-specific, rod, & -1.17 & 0.00 & 0.00 \\
\hline CYP2U1 & 226393_PM_at & cytochrome P450, family 2 , subfamily $U$, po & -1.17 & 0.00 & 0.00 \\
\hline MCM9 & 213561_PM_at & minichromosome maintenance complex co & -1.17 & 0.00 & 0.00 \\
\hline ATXN3 & 205415_PM_s_at & ataxin 3 & -1.17 & 0.00 & 0.00 \\
\hline ANKRD36 & 214723_PM_x_at & ankyrin repeat domain 36 & -1.17 & 0.00 & 0.00 \\
\hline KLHL10 & 1552425_PM_a_at & kelch-like 10 (Drosophila) & -1.17 & 0.00 & 0.00 \\
\hline--- & 1564424_PM_at & --- & -1.17 & 0.00 & 0.00 \\
\hline SBDS & 1554089_PM_s_at & Shwachman-Bodian-Diamond syndrome & -1.17 & 0.00 & 0.00 \\
\hline C9orf91 & 221865_PM_at & chromosome 9 open reading frame 91 & -1.17 & 0.00 & 0.00 \\
\hline ITM2C & 221004_PM_s_at & integral membrane protein $2 \mathrm{C}$ & -1.17 & 0.00 & 0.00 \\
\hline GPATCH4 & 224632_PM_at & G patch domain containing 4 & -1.17 & 0.00 & 0.00 \\
\hline
\end{tabular}




\begin{tabular}{|c|c|c|c|c|c|}
\hline CATSPER2 & 217588_PM_at & cation channel, sperm associated 2 & -1.17 & 0.00 & 0.00 \\
\hline--- & 238172_PM_at & --- & -1.17 & 0.00 & 0.00 \\
\hline--- & 233925_PM_at & --- & -1.17 & 0.00 & 0.00 \\
\hline TRIO & 231403_PM_at & triple functional domain (PTPRF interacting) & -1.17 & 0.00 & 0.00 \\
\hline MCF2L & 35147_PM_at & MCF. 2 cell line derived transforming sequer & -1.17 & 0.00 & 0.00 \\
\hline SETBP1 & 205933_PM_at & SET binding protein 1 & -1.17 & 0.00 & 0.00 \\
\hline--- & 235705_PM_at & --- & -1.17 & 0.00 & 0.00 \\
\hline--- & 1565601_PM_at & --- & -1.17 & 0.00 & 0.00 \\
\hline C9orf85 & 235866_PM_at & chromosome 9 open reading frame 85 & -1.17 & 0.00 & 0.00 \\
\hline AAGAB & 243284_PM_at & alpha- and gamma-adaptin binding protein & -1.17 & 0.00 & 0.00 \\
\hline--- & 243310_PM_at & --- & -1.17 & 0.00 & 0.00 \\
\hline BIN1 & 210201_PM_x_at & bridging integrator 1 & -1.17 & 0.00 & 0.00 \\
\hline--- & 233271_PM_at & --- & -1.17 & 0.00 & 0.00 \\
\hline HEG1 & 213069_PM_at & HEG homolog 1 (zebrafish) & -1.17 & 0.00 & 0.00 \\
\hline QRSL1 & 218949_PM_s_at & glutaminyl-tRNA synthase (glutamine-hydrc & -1.17 & 0.00 & 0.00 \\
\hline SLC12A3 & 215274_PM_at & solute carrier family 12 (sodium & -1.17 & 0.00 & 0.00 \\
\hline TOX4 & 232097_PM_at & TOX high mobility group box family membe & -1.17 & 0.00 & 0.00 \\
\hline ITPR1 & 240052_PM_at & inositol 1,4,5-trisphosphate receptor, type: & -1.17 & 0.00 & 0.00 \\
\hline NME7 & 219553_PM_at & NME & -1.17 & 0.00 & 0.00 \\
\hline--- & 230713_PM_at & --- & -1.17 & 0.00 & 0.01 \\
\hline TRMT10B & 240166_PM_x_at & tRNA methyltransferase 10 homolog B (S. Cl & -1.17 & 0.00 & 0.01 \\
\hline--- & 238744_PM_at & --- & -1.17 & 0.00 & 0.01 \\
\hline HIP1R & 38340_PM_at & huntingtin interacting protein 1 related & -1.17 & 0.00 & 0.01 \\
\hline--- & 240169_PM_at & --- & -1.17 & 0.00 & 0.01 \\
\hline $\mathrm{CHIC1}$ & 228345_PM_at & cysteine-rich hydrophobic domain 1 & -1.17 & 0.00 & 0.01 \\
\hline EP400NL & 229892_PM_at & EP400 N-terminal like & -1.17 & 0.00 & 0.01 \\
\hline SNX29 & 1558745_PM_at & sorting nexin 29 & -1.17 & 0.00 & 0.01 \\
\hline LOC100131043 & 244029_PM_at & uncharacterized LOC100131043 & -1.17 & 0.00 & 0.01 \\
\hline RASGRF2 & 1556128_PM_a_at & Ras protein-specific guanine nucleotide-rel $\epsilon$ & -1.17 & 0.00 & 0.01 \\
\hline--- & 1558686_PM_at & --- & -1.17 & 0.00 & 0.01 \\
\hline NBAS & 242049_PM_s_at & neuroblastoma amplified sequence & -1.17 & 0.00 & 0.01 \\
\hline PTCD3 & 228590_PM_at & pentatricopeptide repeat domain 3 & -1.17 & 0.00 & 0.01 \\
\hline PLCG1 & 202789_PM_at & phospholipase C, gamma 1 & -1.17 & 0.00 & 0.01 \\
\hline
\end{tabular}




\begin{tabular}{|c|c|c|c|c|c|}
\hline ZNF519 & 1564190_PM_x_at & zinc finger protein 519 & -1.17 & 0.00 & 0.01 \\
\hline--- & 231165_PM_at & --- & -1.17 & 0.00 & 0.01 \\
\hline AFAP1L2 & 226829_PM_at & actin filament associated protein 1-like 2 & -1.17 & 0.00 & 0.01 \\
\hline--- & 240743_PM_at & --- & -1.17 & 0.00 & 0.01 \\
\hline 6-Sep & 212413_PM_at & septin 6 & -1.17 & 0.00 & 0.01 \\
\hline--- & 241917_PM_at & --- & -1.17 & 0.00 & 0.01 \\
\hline--- & 222368_PM_at & --- & -1.17 & 0.00 & 0.01 \\
\hline CCAR1 & 224736_PM_at & cell division cycle and apoptosis regulator 1 & -1.16 & 0.00 & 0.00 \\
\hline BOLA2 & 231500_PM_s_at & BolA homolog 2 (E. coli) & -1.16 & 0.00 & 0.00 \\
\hline VAV2 & 226063_PM_at & vav 2 guanine nucleotide exchange factor & -1.16 & 0.00 & 0.00 \\
\hline LUC7L3 & 208835_PM_s_at & LUC7-like 3 (S. cerevisiae) & -1.16 & 0.00 & 0.00 \\
\hline EXOSC9 & 205061_PM_s_at & exosome component 9 & -1.16 & 0.00 & 0.00 \\
\hline IRF8 & 204057_PM_at & interferon regulatory factor 8 & -1.16 & 0.00 & 0.00 \\
\hline ZNF224 & 232427_PM_at & Zinc finger protein 224 & -1.16 & 0.00 & 0.00 \\
\hline HNRPDL & 1554678_PM_s_at & heterogeneous nuclear ribonucleoprotein [ & -1.16 & 0.00 & 0.00 \\
\hline--- & 217464_PM_at & --- & -1.16 & 0.00 & 0.00 \\
\hline--- & 216668_PM_at & --- & -1.16 & 0.00 & 0.00 \\
\hline SRSF7 & 213649_PM_at & serine & -1.16 & 0.00 & 0.00 \\
\hline ALDH18A1 & 217791_PM_s_at & aldehyde dehydrogenase 18 family, memb€ & -1.16 & 0.00 & 0.00 \\
\hline TCF3 & 210776_PM_x_at & transcription factor 3 (E2A immunoglobulin & -1.16 & 0.00 & 0.00 \\
\hline PATZ1 & 209431_PM_s_at & POZ (BTB) and AT hook containing zinc fingt & -1.16 & 0.00 & 0.00 \\
\hline HPS4 & 54037_PM_at & Hermansky-Pudlak syndrome 4 & -1.16 & 0.00 & 0.00 \\
\hline BZW2 & 217809_PM_at & basic leucine zipper and W2 domains 2 & -1.16 & 0.00 & 0.00 \\
\hline--- & 240241_PM_at & --- & -1.16 & 0.00 & 0.00 \\
\hline SYNRG & 222715_PM_s_at & synergin, gamma & -1.16 & 0.00 & 0.00 \\
\hline ZBTB39 & 205256_PM_at & zinc finger and BTB domain containing 39 & -1.16 & 0.00 & 0.00 \\
\hline GNAS & 228173_PM_at & GNAS complex locus & -1.16 & 0.00 & 0.00 \\
\hline DOCK10 & 219279_PM_at & dedicator of cytokinesis 10 & -1.16 & 0.00 & 0.00 \\
\hline--- & 234283_PM_at & --- & -1.16 & 0.00 & 0.00 \\
\hline--- & 1556434_PM_at & --- & -1.16 & 0.00 & 0.00 \\
\hline SIM2 & 206558_PM_at & single-minded homolog 2 (Drosophila) & -1.16 & 0.00 & 0.00 \\
\hline --- & 230461_PM_s_at & --- & -1.16 & 0.00 & 0.00 \\
\hline--- & 1556322_PM_a_at & --- & -1.16 & 0.00 & 0.00 \\
\hline
\end{tabular}




\begin{tabular}{|c|c|c|c|c|c|}
\hline PRKCl & 209678_PM_s_at & protein kinase $\mathrm{C}$, iota & -1.16 & 0.00 & 0.00 \\
\hline KCTD6 & 238077_PM_at & potassium channel tetramerisation domain & -1.16 & 0.00 & 0.00 \\
\hline--- & 1557522_PM_x_at & --- & -1.16 & 0.00 & 0.00 \\
\hline--- & 238277_PM_at & --- & -1.16 & 0.00 & 0.00 \\
\hline CHMP7 & 212313_PM_at & charged multivesicular body protein 7 & -1.16 & 0.00 & 0.00 \\
\hline--- & 1557521_PM_a_at & --- & -1.16 & 0.00 & 0.00 \\
\hline METTL17 & 218366_PM_x_at & methyltransferase like 17 & -1.16 & 0.00 & 0.00 \\
\hline WBSCR22 & 207628_PM_s_at & Williams Beuren syndrome chromosome re & -1.16 & 0.00 & 0.00 \\
\hline--- & 236066_PM_at & --- & -1.16 & 0.00 & 0.00 \\
\hline MAGEE1 & 229286_PM_at & melanoma antigen family $E, 1$ & -1.16 & 0.00 & 0.00 \\
\hline--- & 224417_PM_at & --- & -1.16 & 0.00 & 0.00 \\
\hline SLC25A17 & 214210_PM_at & solute carrier family 25 (mitochondrial carri & -1.16 & 0.00 & 0.00 \\
\hline BTN3A3 & 38241_PM_at & butyrophilin, subfamily 3 , member $A 3$ & -1.16 & 0.00 & 0.00 \\
\hline--- & 243329_PM_at & --- & -1.16 & 0.00 & 0.00 \\
\hline--- & 215974_PM_at & --- & -1.16 & 0.00 & 0.00 \\
\hline--- & 236685_PM_at & --- & -1.16 & 0.00 & 0.00 \\
\hline CTPS2 & 222819_PM_at & CTP synthase 2 & -1.16 & 0.00 & 0.00 \\
\hline--- & 233114_PM_at & --- & -1.16 & 0.00 & 0.00 \\
\hline RBM17 & 225751_PM_at & RNA binding motif protein 17 & -1.16 & 0.00 & 0.00 \\
\hline CHEK2 & 210416_PM_s_at & checkpoint kinase 2 & -1.16 & 0.00 & 0.00 \\
\hline OSGEPL1 & 230032_PM_at & O-sialoglycoprotein endopeptidase-like 1 & -1.16 & 0.00 & 0.00 \\
\hline ST3GAL5 & 203217_PM_s_at & ST3 beta-galactoside alpha-2,3-sialyltransfe & -1.16 & 0.00 & 0.00 \\
\hline DNASE1L3 & 1561336_PM_at & deoxyribonuclease I-like 3 & -1.16 & 0.00 & 0.00 \\
\hline--- & 237180_PM_at & --- & -1.16 & 0.00 & 0.00 \\
\hline ZNF789 & 1569194_PM_at & zinc finger protein 789 & -1.16 & 0.00 & 0.00 \\
\hline AP4S1 & 210278_PM_s_at & adaptor-related protein complex 4, sigma 1 & -1.16 & 0.00 & 0.00 \\
\hline SPG7 & 230884_PM_s_at & spastic paraplegia 7 (pure and complicated & -1.16 & 0.00 & 0.00 \\
\hline SLC2A4RG & 1555500_PM_s_at & SLC2A4 regulator & -1.16 & 0.00 & 0.00 \\
\hline KAT6B & 1562236_PM_at & $\mathrm{K}$ (lysine) acetyltransferase 6B & -1.16 & 0.00 & 0.00 \\
\hline ZNF346 & 236267_PM_at & zinc finger protein 346 & -1.16 & 0.00 & 0.00 \\
\hline--- & 243524_PM_at & --- & -1.16 & 0.00 & 0.00 \\
\hline --- & 236776_PM_at & --- & -1.16 & 0.00 & 0.00 \\
\hline GKAP1 & 229312_PM_s_at & G kinase anchoring protein 1 & -1.16 & 0.00 & 0.00 \\
\hline
\end{tabular}




\begin{tabular}{|c|c|c|c|c|c|}
\hline--- & 240393_PM_at & --- & -1.16 & 0.00 & 0.00 \\
\hline SFXN2 & 227560_PM_at & sideroflexin 2 & -1.16 & 0.00 & 0.00 \\
\hline ETS1 & 214447_PM_at & v-ets erythroblastosis virus E26 oncogene $h$ & -1.16 & 0.00 & 0.00 \\
\hline LINC00094 & 203245_PM_s_at & long intergenic non-protein coding RNA 94 & -1.16 & 0.00 & 0.00 \\
\hline SIDT1 & 219734_PM_at & SID1 transmembrane family, member 1 & -1.16 & 0.00 & 0.00 \\
\hline IREB2 & 242261_PM_at & iron-responsive element binding protein 2 & -1.16 & 0.00 & 0.00 \\
\hline TAF15 & 202840_PM_at & TAF15 RNA polymerase II, TATA box binding & -1.16 & 0.00 & 0.00 \\
\hline USP24 & 230004_PM_at & ubiquitin specific peptidase 24 & -1.16 & 0.00 & 0.00 \\
\hline TTC9C & 1569189_PM_at & tetratricopeptide repeat domain $9 \mathrm{C}$ & -1.16 & 0.00 & 0.00 \\
\hline GPR157 & 227970_PM_at & G protein-coupled receptor 157 & -1.16 & 0.00 & 0.00 \\
\hline--- & 241965_PM_at & --- & -1.16 & 0.00 & 0.00 \\
\hline CCDC90B & 1568834_PM_s_at & coiled-coil domain containing 90B & -1.16 & 0.00 & 0.00 \\
\hline--- & 1558739_PM_at & --- & -1.16 & 0.00 & 0.00 \\
\hline BCL2L13 & 1560977_PM_a_at & BCL2-like 13 (apoptosis facilitator) & -1.16 & 0.00 & 0.00 \\
\hline--- & 238666_PM_at & --- & -1.16 & 0.00 & 0.00 \\
\hline--- & 229896_PM_at & --- & -1.16 & 0.00 & 0.00 \\
\hline $\mathrm{CHCHD10}$ & 224932_PM_at & coiled-coil-helix-coiled-coil-helix domain col & -1.16 & 0.00 & 0.00 \\
\hline DLG1 & 230229_PM_at & Discs, large homolog 1 (Drosophila) & -1.16 & 0.00 & 0.00 \\
\hline GLMN & 207153_PM_s_at & glomulin, FKBP associated protein & -1.16 & 0.00 & 0.00 \\
\hline HMGN3 & 209377_PM_s_at & high mobility group nucleosomal binding dc & -1.16 & 0.00 & 0.00 \\
\hline PSPC1 & 222611_PM_s_at & paraspeckle component 1 & -1.16 & 0.00 & 0.00 \\
\hline MLL & 220546_PM_at & myeloid & -1.16 & 0.00 & 0.00 \\
\hline SP140L & 214791_PM_at & SP140 nuclear body protein-like & -1.16 & 0.00 & 0.00 \\
\hline ARMCX5-GPRASP2 & 228027_PM_at & ARMCX5-GPRASP2 readthrough & -1.16 & 0.00 & 0.00 \\
\hline RNF8 & 203160_PM_s_at & ring finger protein 8 , E3 ubiquitin protein li६ & -1.16 & 0.00 & 0.00 \\
\hline MPHOSPH6 & 203740_PM_at & M-phase phosphoprotein 6 & -1.16 & 0.00 & 0.00 \\
\hline NUCKS1 & 223661_PM_at & Nuclear casein kinase and cyclin-dependent & -1.16 & 0.00 & 0.00 \\
\hline $\mathrm{MDH} 1$ & 235374_PM_at & Malate dehydrogenase 1, NAD (soluble) & -1.16 & 0.00 & 0.00 \\
\hline--- & 240769_PM_at & --- & -1.16 & 0.00 & 0.00 \\
\hline BIN1 & 202931_PM_x_at & bridging integrator 1 & -1.16 & 0.00 & 0.00 \\
\hline--- & 240481_PM_at & --- & -1.16 & 0.00 & 0.00 \\
\hline XPO1 & 235927_PM_at & exportin 1 (CRM1 homolog, yeast) & -1.16 & 0.00 & 0.00 \\
\hline NDUFB4 & 228940_PM_at & NADH dehydrogenase (ubiquinone) 1 beta & -1.16 & 0.00 & 0.00 \\
\hline
\end{tabular}




\begin{tabular}{|c|c|c|c|c|c|}
\hline DKFZP434C153 & 216085_PM_at & DKFZP434C153 protein & -1.16 & 0.00 & 0.00 \\
\hline--- & 240321_PM_at & --- & -1.16 & 0.00 & 0.00 \\
\hline--- & 232511_PM_at & --- & -1.16 & 0.00 & 0.00 \\
\hline RALGAPA1 & 215162_PM_at & Ral GTPase activating protein, alpha subuni & -1.16 & 0.00 & 0.00 \\
\hline--- & 236969_PM_at & --- & -1.16 & 0.00 & 0.00 \\
\hline LOC642852 & 1559957_PM_a_at & uncharacterized LOC642852 & -1.16 & 0.00 & 0.00 \\
\hline TTN & 1557994_PM_at & titin & -1.16 & 0.00 & 0.00 \\
\hline ZNF326 & 241720_PM_at & zinc finger protein 326 & -1.16 & 0.00 & 0.00 \\
\hline ZAP70 & 1555613_PM_a_at & zeta-chain (TCR) associated protein kinase $\bar{i}$ & -1.16 & 0.00 & 0.00 \\
\hline UTP20 & 209725_PM_at & UTP20, small subunit (SSU) processome cor & -1.16 & 0.00 & 0.00 \\
\hline CEP68 & 239442_PM_at & centrosomal protein $68 \mathrm{kDa}$ & -1.16 & 0.00 & 0.00 \\
\hline LOC92249 & 216838_PM_at & uncharacterized LOC92249 & -1.16 & 0.00 & 0.01 \\
\hline PIEZO1 & 1566111_PM_at & piezo-type mechanosensitive ion channel cr & -1.16 & 0.00 & 0.01 \\
\hline ACAD10 & 237481_PM_at & acyl-CoA dehydrogenase family, member 1( & -1.16 & 0.00 & 0.01 \\
\hline DMRTC1 & 1553998_PM_at & DMRT-like family C1 & -1.16 & 0.00 & 0.01 \\
\hline CYP2E1 & 209975_PM_at & cytochrome P450, family 2 , subfamily E, pol & -1.16 & 0.00 & 0.01 \\
\hline RIF1 & 241820_PM_at & RAP1 interacting factor homolog (yeast) & -1.16 & 0.00 & 0.01 \\
\hline COG2 & 243608_PM_at & component of oligomeric golgi complex 2 & -1.16 & 0.00 & 0.01 \\
\hline CLNS1A & 242492_PM_at & chloride channel, nucleotide-sensitive, $1 \mathrm{~A}$ & -1.16 & 0.00 & 0.01 \\
\hline--- & 242223_PM_at & --- & -1.16 & 0.00 & 0.01 \\
\hline ZDHHC2 & 1563502_PM_at & Zinc finger, DHHC-type containing 2 & -1.16 & 0.00 & 0.01 \\
\hline NUP107 & 218768_PM_at & nucleoporin $107 \mathrm{kDa}$ & -1.16 & 0.00 & 0.01 \\
\hline--- & 243305_PM_at & --- & -1.16 & 0.00 & 0.01 \\
\hline--- & 243262_PM_at & --- & -1.16 & 0.00 & 0.01 \\
\hline TRNAU1AP & 228997_PM_at & tRNA selenocysteine 1 associated protein 1 & -1.16 & 0.00 & 0.01 \\
\hline--- & 235662_PM_at & --- & -1.16 & 0.00 & 0.01 \\
\hline CNOT4 & 210866_PM_s_at & CCR4-NOT transcription complex, subunit 4 & -1.16 & 0.00 & 0.01 \\
\hline GTF3A & 238880_PM_at & general transcription factor IIIA & -1.16 & 0.00 & 0.01 \\
\hline BIN1 & 214439_PM_x_at & bridging integrator 1 & -1.16 & 0.00 & 0.01 \\
\hline AGPAT3 & 223182_PM_s_at & 1-acylglycerol-3-phosphate O-acyltransfera: & -1.16 & 0.00 & 0.01 \\
\hline STRBP & 223246_PM_s_at & spermatid perinuclear RNA binding protein & -1.16 & 0.00 & 0.01 \\
\hline ZNF275 & 225382_PM_at & zinc finger protein 275 & -1.16 & 0.00 & 0.01 \\
\hline HCG18 & 225584_PM_at & HLA complex group 18 (non-protein coding) & -1.16 & 0.00 & 0.01 \\
\hline
\end{tabular}




\begin{tabular}{|c|c|c|c|c|c|}
\hline ZNF280B & 229360_PM_at & zinc finger protein $280 \mathrm{~B}$ & -1.16 & 0.00 & 0.01 \\
\hline--- & 238944_PM_at & --- & -1.16 & 0.00 & 0.01 \\
\hline--- & 221038_PM_at & --- & -1.16 & 0.00 & 0.01 \\
\hline--- & 232974_PM_at & --- & -1.16 & 0.00 & 0.01 \\
\hline--- & 237317_PM_at & --- & -1.16 & 0.00 & 0.01 \\
\hline ATP6V1H & 1557586_PM_s_at & ATPase, $\mathrm{H}+$ transporting, lysosomal 50 & -1.16 & 0.00 & 0.01 \\
\hline POU2F2 & 228343_PM_at & POU class 2 homeobox 2 & -1.16 & 0.00 & 0.01 \\
\hline LOC338758 & 238893_PM_at & uncharacterized LOC338758 & -1.16 & 0.00 & 0.01 \\
\hline--- & 215635_PM_at & --- & -1.16 & 0.00 & 0.01 \\
\hline PNISR & 212177_PM_at & PNN-interacting serine & -1.15 & 0.00 & 0.00 \\
\hline--- & 1559054_PM_a_at & --- & -1.15 & 0.00 & 0.00 \\
\hline TMEM101 & 225004_PM_at & transmembrane protein 101 & -1.15 & 0.00 & 0.00 \\
\hline RFX5 & 202963_PM_at & regulatory factor $X, 5$ (influences HLA class I & -1.15 & 0.00 & 0.00 \\
\hline PNPO & 218511_PM_s_at & pyridoxamine 5'-phosphate oxidase & -1.15 & 0.00 & 0.00 \\
\hline C16orf52 & 230296_PM_at & chromosome 16 open reading frame 52 & -1.15 & 0.00 & 0.00 \\
\hline KLHL28 & 235727_PM_at & kelch-like 28 (Drosophila) & -1.15 & 0.00 & 0.00 \\
\hline SMARCE1 & 211989_PM_at & SWI & -1.15 & 0.00 & 0.00 \\
\hline RCN1 & 201063_PM_at & reticulocalbin 1, EF-hand calcium binding dc & -1.15 & 0.00 & 0.00 \\
\hline FUS & 217370_PM_x_at & fused in sarcoma & -1.15 & 0.00 & 0.00 \\
\hline--- & 213158_PM_at & --- & -1.15 & 0.00 & 0.00 \\
\hline--- & 243631_PM_at & --- & -1.15 & 0.00 & 0.00 \\
\hline NPIPL3 & 215920_PM_s_at & nuclear pore complex interacting protein-lil & -1.15 & 0.00 & 0.00 \\
\hline--- & 229483_PM_at & --- & -1.15 & 0.00 & 0.00 \\
\hline NKTR & 215338_PM_s_at & natural killer-tumor recognition sequence & -1.15 & 0.00 & 0.00 \\
\hline PSMG4 & 233443_PM_at & proteasome (prosome, macropain) assembl & -1.15 & 0.00 & 0.00 \\
\hline SLC25A26 & 225862_PM_at & solute carrier family 25 (S-adenosylmethion & -1.15 & 0.00 & 0.00 \\
\hline--- & 227082_PM_at & --- & -1.15 & 0.00 & 0.00 \\
\hline AKR7A2 & 214259_PM_s_at & aldo-keto reductase family 7 , member $A 2$ ( $\bar{c}$ & -1.15 & 0.00 & 0.00 \\
\hline--- & 244778_PM_x_at & --- & -1.15 & 0.00 & 0.00 \\
\hline TMEM161B & 238783_PM_at & transmembrane protein 161B & -1.15 & 0.00 & 0.00 \\
\hline--- & 213156_PM_at & --- & -1.15 & 0.00 & 0.00 \\
\hline $\mathrm{LBH}$ & 221011_PM_s_at & limb bud and heart development homolog ( & -1.15 & 0.00 & 0.00 \\
\hline $\mathrm{BICD} 1$ & 1556051_PM_a_at & bicaudal D homolog 1 (Drosophila) & -1.15 & 0.00 & 0.00 \\
\hline
\end{tabular}




\begin{tabular}{|c|c|c|c|c|c|}
\hline AGK & 222132_PM_s_at & acylglycerol kinase & -1.15 & 0.00 & 0.00 \\
\hline ATP9B & 214934_PM_at & ATPase, class II, type 9B & -1.15 & 0.00 & 0.00 \\
\hline--- & 243091_PM_at & --- & -1.15 & 0.00 & 0.00 \\
\hline--- & 243745_PM_at & --- & -1.15 & 0.00 & 0.00 \\
\hline RGS10 & 204316_PM_at & regulator of G-protein signaling 10 & -1.15 & 0.00 & 0.00 \\
\hline--- & 1558740_PM_s_at & --- & -1.15 & 0.00 & 0.00 \\
\hline PDIA4 & 208658_PM_at & protein disulfide isomerase family $A$, memb & -1.15 & 0.00 & 0.00 \\
\hline LINC00338 & 221621_PM_at & long intergenic non-protein coding RNA $33 \varepsilon$ & -1.15 & 0.00 & 0.00 \\
\hline PLK1S1 & 233241_PM_at & polo-like kinase 1 substrate 1 & -1.15 & 0.00 & 0.00 \\
\hline GPC2 & 239422_PM_at & glypican 2 & -1.15 & 0.00 & 0.00 \\
\hline--- & 236784_PM_s_at & --- & -1.15 & 0.00 & 0.00 \\
\hline--- & 239549_PM_at & --- & -1.15 & 0.00 & 0.00 \\
\hline--- & 228611_PM_s_at & --- & -1.15 & 0.00 & 0.00 \\
\hline PDE12 & 1554915_PM_a_at & phosphodiesterase 12 & -1.15 & 0.00 & 0.00 \\
\hline MKLN1 & 1560145_PM_at & Muskelin 1, intracellular mediator containir & -1.15 & 0.00 & 0.00 \\
\hline--- & 236054_PM_at & --- & -1.15 & 0.00 & 0.00 \\
\hline RABL2A & 219151_PM_s_at & RAB, member of RAS oncogene family-like $z$ & -1.15 & 0.00 & 0.00 \\
\hline SCLY & 221575_PM_at & selenocysteine lyase & -1.15 & 0.00 & 0.00 \\
\hline MIS18A & 229671_PM_s_at & MIS18 kinetochore protein homolog A (S. p & -1.15 & 0.00 & 0.00 \\
\hline--- & 242972_PM_at & --- & -1.15 & 0.00 & 0.00 \\
\hline POLR3E & 218016_PM_s_at & polymerase (RNA) III (DNA directed) polype & -1.15 & 0.00 & 0.00 \\
\hline--- & 244002_PM_at & --- & -1.15 & 0.00 & 0.00 \\
\hline RABGAP1 & 204028_PM_s_at & RAB GTPase activating protein 1 & -1.15 & 0.00 & 0.00 \\
\hline RRN3 & 230328_PM_at & RRN3 RNA polymerase I transcription factor & -1.15 & 0.00 & 0.00 \\
\hline MDC1 & 203062_PM_s_at & mediator of DNA-damage checkpoint 1 & -1.15 & 0.00 & 0.00 \\
\hline--- & 243217_PM_at & --- & -1.15 & 0.00 & 0.00 \\
\hline--- & 1562673_PM_at & --- & -1.15 & 0.00 & 0.00 \\
\hline DAAM1 & 244062_PM_at & dishevelled associated activator of morpho & -1.15 & 0.00 & 0.00 \\
\hline DFFB & 206752_PM_s_at & DNA fragmentation factor, $40 \mathrm{kDa}$, beta pol) & -1.15 & 0.00 & 0.00 \\
\hline VPS13C & 235023_PM_at & Vacuolar protein sorting 13 homolog C (S. c & -1.15 & 0.00 & 0.00 \\
\hline LUZP1 & 221832_PM_s_at & leucine zipper protein 1 & -1.15 & 0.00 & 0.00 \\
\hline ANKRD40 & 227064_PM_at & ankyrin repeat domain 40 & -1.15 & 0.00 & 0.00 \\
\hline ARHGEF7 & 229642_PM_at & Rho guanine nucleotide exchange factor (G) & -1.15 & 0.00 & 0.00 \\
\hline
\end{tabular}




\begin{tabular}{|c|c|c|c|c|c|}
\hline METTL2A & 225253_PM_s_at & methyltransferase like $2 \mathrm{~A}$ & -1.15 & 0.00 & 0.00 \\
\hline MPHOSPH10 & 212885_PM_at & M-phase phosphoprotein 10 (U3 small nucl & -1.15 & 0.00 & 0.00 \\
\hline FNBP1 & 230086_PM_at & formin binding protein 1 & -1.15 & 0.00 & 0.00 \\
\hline HUWE1 & 214673_PM_s_at & HECT, UBA and WWE domain containing 1 , & -1.15 & 0.00 & 0.00 \\
\hline TP73-AS1 & 213340_PM_s_at & TP73 antisense RNA 1 (non-protein coding) & -1.15 & 0.00 & 0.00 \\
\hline PMS2L2 & 214757_PM_at & postmeiotic segregation increased 2 -like $2 \mathrm{k}$ & -1.15 & 0.00 & 0.00 \\
\hline--- & 1570318_PM_at & --- & -1.15 & 0.00 & 0.00 \\
\hline PCNT & 233387_PM_s_at & pericentrin & -1.15 & 0.00 & 0.00 \\
\hline TMEM201 & 228671_PM_at & transmembrane protein 201 & -1.15 & 0.00 & 0.00 \\
\hline--- & 240139_PM_at & --- & -1.15 & 0.00 & 0.00 \\
\hline SIK2 & 213221_PM_s_at & salt-inducible kinase 2 & -1.15 & 0.00 & 0.00 \\
\hline SNX5 & 229981_PM_at & sorting nexin 5 & -1.15 & 0.00 & 0.00 \\
\hline FNIP1 & 1555247_PM_a_at & folliculin interacting protein 1 & -1.15 & 0.00 & 0.00 \\
\hline RUVBL1 & 201614_PM_s_at & RuvB-like 1 (E. coli) & -1.15 & 0.00 & 0.00 \\
\hline--- & 1556479_PM_at & --- & -1.15 & 0.00 & 0.00 \\
\hline KIF5C & 203129_PM_s_at & kinesin family member $5 \mathrm{C}$ & -1.15 & 0.00 & 0.00 \\
\hline ANKH & 223092_PM_at & ankylosis, progressive homolog (mouse) & -1.15 & 0.00 & 0.00 \\
\hline XPO7 & 240168_PM_at & exportin 7 & -1.15 & 0.00 & 0.00 \\
\hline BBS2 & 223227_PM_at & Bardet-Biedl syndrome 2 & -1.15 & 0.00 & 0.00 \\
\hline--- & 241089_PM_at & --- & -1.15 & 0.00 & 0.00 \\
\hline PTPN2 & 241622_PM_at & Protein tyrosine phosphatase, non-receptol & -1.15 & 0.00 & 0.00 \\
\hline NDNL2 & 226008_PM_at & necdin-like 2 & -1.15 & 0.00 & 0.00 \\
\hline--- & 213484_PM_at & --- & -1.15 & 0.00 & 0.00 \\
\hline KRR1 & 232441_PM_at & KRR1, small subunit (SSU) processome com| & -1.15 & 0.00 & 0.00 \\
\hline EPM2A & 205231_PM_s_at & epilepsy, progressive myoclonus type $2 \mathrm{~A}, \mathrm{~L} \bar{c}$ & -1.15 & 0.00 & 0.00 \\
\hline FABP3 & 214285_PM_at & fatty acid binding protein 3 , muscle and hé & -1.15 & 0.00 & 0.00 \\
\hline NAPEPLD & 242229_PM_at & $\mathrm{N}$-acyl phosphatidylethanolamine phosphol & -1.15 & 0.00 & 0.00 \\
\hline RPS28 & 208903_PM_at & ribosomal protein S28 & -1.15 & 0.00 & 0.00 \\
\hline CLYBL & 238440_PM_at & citrate lyase beta like & -1.15 & 0.00 & 0.00 \\
\hline MDM4 & 205655_PM_at & Mdm4 p53 binding protein homolog (mous & -1.15 & 0.00 & 0.00 \\
\hline PTCD3 & 228512_PM_at & pentatricopeptide repeat domain 3 & -1.15 & 0.00 & 0.00 \\
\hline --- & 215630_PM_at & --- & -1.15 & 0.00 & 0.00 \\
\hline --- & 222352_PM_at & --- & -1.15 & 0.00 & 0.00 \\
\hline
\end{tabular}




\begin{tabular}{|c|c|c|c|c|c|}
\hline--- & 234649_PM_at & --- & -1.15 & 0.00 & 0.00 \\
\hline--- & 244298_PM_at & --- & -1.15 & 0.00 & 0.00 \\
\hline--- & 235493_PM_at & --- & -1.15 & 0.00 & 0.00 \\
\hline RPS16 & 226130_PM_at & ribosomal protein S16 & -1.15 & 0.00 & 0.00 \\
\hline LOC100653024 & 208820_PM_at & uncharacterized LOC100653024 & -1.15 & 0.00 & 0.00 \\
\hline ZMYND8 & 230533_PM_at & zinc finger, MYND-type containing 8 & -1.15 & 0.00 & 0.00 \\
\hline--- & 239160_PM_at & --- & -1.15 & 0.00 & 0.00 \\
\hline--- & 242696_PM_at & --- & -1.15 & 0.00 & 0.00 \\
\hline $\mathrm{ABI} 2$ & 216113_PM_at & Abl-interactor 2 & -1.15 & 0.00 & 0.00 \\
\hline PRDM15 & 231931_PM_at & PR domain containing 15 & -1.15 & 0.00 & 0.00 \\
\hline YLPM1 & 1557065_PM_at & YLP motif containing 1 & -1.15 & 0.00 & 0.00 \\
\hline--- & 238303_PM_at & --- & -1.15 & 0.00 & 0.00 \\
\hline 6-Sep & 1555526_PM_a_at & septin 6 & -1.15 & 0.00 & 0.00 \\
\hline C1orf109 & 218712_PM_at & chromosome 1 open reading frame 109 & -1.15 & 0.00 & 0.00 \\
\hline--- & 236586_PM_at & --- & -1.15 & 0.00 & 0.00 \\
\hline PPIH & 204228_PM_at & peptidylprolyl isomerase $\mathrm{H}$ (cyclophilin $\mathrm{H}$ ) & -1.15 & 0.00 & 0.00 \\
\hline CD6 & 1566448_PM_at & CD6 molecule & -1.15 & 0.00 & 0.00 \\
\hline SPTBN1 & 200672_PM_x_at & spectrin, beta, non-erythrocytic 1 & -1.15 & 0.00 & 0.00 \\
\hline PDCD5 & 227751_PM_at & programmed cell death 5 & -1.15 & 0.00 & 0.01 \\
\hline SMURF2 & 227489_PM_at & SMAD specific E3 ubiquitin protein ligase 2 & -1.15 & 0.00 & 0.01 \\
\hline RTN4IP1 & 1555679_PM_a_at & reticulon 4 interacting protein 1 & -1.15 & 0.00 & 0.01 \\
\hline--- & 1557196_PM_a_at & --- & -1.15 & 0.00 & 0.01 \\
\hline NUB1 & 1557071_PM_s_at & negative regulator of ubiquitin-like proteins & -1.15 & 0.00 & 0.01 \\
\hline--- & 232700_PM_at & --- & -1.15 & 0.00 & 0.01 \\
\hline COQ10A & 225747_PM_at & coenzyme Q10 homolog A (S. cerevisiae) & -1.15 & 0.00 & 0.01 \\
\hline--- & 1569703_PM_a_at & --- & -1.15 & 0.00 & 0.01 \\
\hline--- & 231605_PM_at & --- & -1.15 & 0.00 & 0.01 \\
\hline CREM & 207630_PM_s_at & cAMP responsive element modulator & -1.15 & 0.00 & 0.01 \\
\hline--- & 228811_PM_at & --- & -1.15 & 0.00 & 0.01 \\
\hline--- & 240307_PM_at & --- & -1.15 & 0.00 & 0.01 \\
\hline HNRNPC & 235500_PM_at & heterogeneous nuclear ribonucleoprotein C & -1.15 & 0.00 & 0.01 \\
\hline PDCD2 & 243773_PM_at & programmed cell death 2 & -1.15 & 0.00 & 0.01 \\
\hline SPICE1 & 234995_PM_at & spindle and centriole associated protein 1 & -1.15 & 0.00 & 0.0 \\
\hline
\end{tabular}




\begin{tabular}{|c|c|c|c|c|c|}
\hline--- & 241849_PM_at & --- & -1.15 & 0.00 & 0.01 \\
\hline --- & 236585_PM_at & --- & -1.15 & 0.00 & 0.01 \\
\hline TBC1D22B & 233430_PM_at & TBC1 domain family, member 22B & -1.15 & 0.00 & 0.01 \\
\hline GLTSCR2 & 234339_PM_S_at & glioma tumor suppressor candidate region & -1.15 & 0.00 & 0.01 \\
\hline SIKE1 & 221705_PM_s_at & suppressor of IKBKE 1 & -1.15 & 0.00 & 0.01 \\
\hline HEATR7A & 232498_PM_at & HEAT repeat containing 7A & -1.15 & 0.00 & 0.01 \\
\hline TAF11 & 1558135_PM_at & TAF11 RNA polymerase II, TATA box bindin & -1.15 & 0.00 & 0.01 \\
\hline MGC21881 & 228040_PM_at & uncharacterized locus MGC21881 & -1.15 & 0.00 & 0.01 \\
\hline SPCS2 & 243570_PM_at & signal peptidase complex subunit 2 homolo & -1.15 & 0.00 & 0.01 \\
\hline --- & 236474_PM_at & --- & -1.15 & 0.00 & 0.01 \\
\hline--- & 240061_PM_at & --- & -1.15 & 0.00 & 0.01 \\
\hline SNX22 & 235460_PM_at & sorting nexin 22 & -1.15 & 0.00 & 0.01 \\
\hline LOC100289019 & 1560089_PM_at & uncharacterized LOC100289019 & -1.15 & 0.00 & 0.01 \\
\hline TAMM41 & 231958 PM at & TAM41, mitochondrial translocator assemb & -1.15 & 0.00 & 0.01 \\
\hline CDC14A & 210742_PM_at & CDC14 cell division cycle 14 homolog A (S. C & -1.15 & 0.00 & 0.01 \\
\hline QRICH1 & 229806_PM_at & Glutamine-rich 1 & -1.14 & 0.00 & 0.00 \\
\hline FBRSL1 & 225703_PM_at & fibrosin-like 1 & -1.14 & 0.00 & 0.00 \\
\hline CCNL2 & 221427 PM S at & cyclin L2 & -1.14 & 0.00 & 0.00 \\
\hline CBFA2T2 & 207625 PM_s_at & core-binding factor, runt domain, alpha sub & -1.14 & 0.00 & 0.00 \\
\hline SLC25A14 & 204587_PM_at & solute carrier family 25 (mitochondrial carri & -1.14 & 0.00 & 0.00 \\
\hline MRPL49 & 201717_PM_at & mitochondrial ribosomal protein $\mathrm{L} 49$ & -1.14 & 0.00 & 0.00 \\
\hline RBM4B & 209497_PM_S_at & RNA binding motif protein $4 \mathrm{~B}$ & -1.14 & 0.00 & 0.00 \\
\hline RPP14 & 204245 PM s at & ribonuclease $\mathrm{P}$ & -1.14 & 0.00 & 0.00 \\
\hline ZNF493 & 240155_PM_x_at & zinc finger protein 493 & -1.14 & 0.00 & 0.00 \\
\hline LOC285033 & 235584_PM_at & uncharacterized LOC285033 & -1.14 & 0.00 & 0.00 \\
\hline SUGP2 & 64371_PM_at & SURP and $G$ patch domain containing 2 & -1.14 & 0.00 & 0.00 \\
\hline AKT2 & $22615 \bar{C}^{-} \mathrm{PM}$ at & v-akt murine thymoma viral oncogene hom & -1.14 & 0.00 & 0.00 \\
\hline ARID4B & $2125911^{-} \mathrm{PM}^{-}$at & AT rich interactive domain $4 \mathrm{~B}$ (RBP1-like) & -1.14 & 0.00 & 0.00 \\
\hline SP140L & 223934_PM_at & SP140 nuclear body protein-like & -1.14 & 0.00 & 0.00 \\
\hline SPAG8 & 206816_PM_S_at & sperm associated antigen 8 & -1.14 & 0.00 & 0.00 \\
\hline KLHL26 & 219354_PM_at & kelch-like 26 (Drosophila) & -1.14 & 0.00 & 0.00 \\
\hline AMN & $1563792^{-} \mathrm{PM}^{-}$at & amnionless homolog (mouse) & -1.14 & 0.00 & 0.00 \\
\hline PRKD3 & 222565_PM_S_at & protein kinase D3 & -1.14 & 0.00 & 0.00 \\
\hline
\end{tabular}




\begin{tabular}{|c|c|c|c|c|c|}
\hline WDR27 & 235576_PM_at & WD repeat domain 27 & -1.14 & 0.00 & 0.00 \\
\hline TNPO2 & 221506_PM_s_at & transportin 2 & -1.14 & 0.00 & 0.00 \\
\hline RAB30 & 206530_PM_at & RAB30, member RAS oncogene family & -1.14 & 0.00 & 0.00 \\
\hline $\mathrm{MTCH} 2$ & 222403_PM_at & mitochondrial carrier 2 & -1.14 & 0.00 & 0.00 \\
\hline SEH1L & 221931_PM_s_at & SEH1-like (S. cerevisiae) & -1.14 & 0.00 & 0.00 \\
\hline SOCS7 & 228662_PM_at & suppressor of cytokine signaling 7 & -1.14 & 0.00 & 0.00 \\
\hline RBM25 & 1557081_PM_at & RNA binding motif protein 25 & -1.14 & 0.00 & 0.00 \\
\hline FLT1 & 232809_PM_s_at & Fms-related tyrosine kinase 1 (vascular end & -1.14 & 0.00 & 0.00 \\
\hline--- & 232937_PM_at & --- & -1.14 & 0.00 & 0.00 \\
\hline SAE1 & 1555618_PM_s_at & SUMO1 activating enzyme subunit 1 & -1.14 & 0.00 & 0.00 \\
\hline FNBP4 & 212232_PM_at & formin binding protein 4 & -1.14 & 0.00 & 0.00 \\
\hline MESDC2 & 224675_PM_at & mesoderm development candidate 2 & -1.14 & 0.00 & 0.00 \\
\hline TMEM41A & 235037_PM_at & transmembrane protein $41 \mathrm{~A}$ & -1.14 & 0.00 & 0.00 \\
\hline UBA5 & 222578_PM_s_at & ubiquitin-like modifier activating enzyme 5 & -1.14 & 0.00 & 0.00 \\
\hline UXS1 & 225583_PM_at & UDP-glucuronate decarboxylase 1 & -1.14 & 0.00 & 0.00 \\
\hline--- & 233235_PM_x_at & --- & -1.14 & 0.00 & 0.00 \\
\hline RUSC1-AS1 & 1552862_PM_at & RUSC1 antisense RNA 1 (non-protein codin६ & -1.14 & 0.00 & 0.00 \\
\hline--- & 241775_PM_at & --- & -1.14 & 0.00 & 0.00 \\
\hline RBM25 & 212027_PM_at & RNA binding motif protein 25 & -1.14 & 0.00 & 0.00 \\
\hline TCF3 & 213730_PM_x_at & transcription factor 3 (E2A immunoglobulin & -1.14 & 0.00 & 0.00 \\
\hline--- & 233274_PM_at & --- & -1.14 & 0.00 & 0.00 \\
\hline HNRNPA2B1 & 205292_PM_s_at & heterogeneous nuclear ribonucleoprotein $A$ & -1.14 & 0.00 & 0.00 \\
\hline--- & 238908_PM_at & --- & -1.14 & 0.00 & 0.00 \\
\hline SMARCC2 & 1561973_PM_at & SWI & -1.14 & 0.00 & 0.00 \\
\hline--- & 239476_PM_at & --- & -1.14 & 0.00 & 0.00 \\
\hline NAP1L1 & 204528_PM_s_at & nucleosome assembly protein 1-like 1 & -1.14 & 0.00 & 0.00 \\
\hline HPS4 & 218402_PM_s_at & Hermansky-Pudlak syndrome 4 & -1.14 & 0.00 & 0.00 \\
\hline UBAP1L & 232557_PM_at & ubiquitin associated protein 1-like & -1.14 & 0.00 & 0.00 \\
\hline TULP3 & 221964_PM_at & tubby like protein 3 & -1.14 & 0.00 & 0.00 \\
\hline LOC254128 & 235132_PM_at & uncharacterized LOC254128 & -1.14 & 0.00 & 0.00 \\
\hline ZNF606 & 229707_PM_at & zinc finger protein 606 & -1.14 & 0.00 & 0.00 \\
\hline TADA2A & 210537_PM_s_at & transcriptional adaptor $2 \mathrm{~A}$ & -1.14 & 0.00 & 0.00 \\
\hline OSGEP & 209450_PM_at & O-sialoglycoprotein endopeptidase & -1.14 & 0.00 & 0.00 \\
\hline
\end{tabular}




\begin{tabular}{|c|c|c|c|c|c|}
\hline MRPS5 & 237560_PM_at & mitochondrial ribosomal protein S5 & -1.14 & 0.00 & 0.00 \\
\hline--- & 240698_PM_s_at & --- & -1.14 & 0.00 & 0.00 \\
\hline PRKCH & 206099_PM_at & protein kinase $\mathrm{C}$, eta & -1.14 & 0.00 & 0.00 \\
\hline--- & 234153_PM_at & --- & -1.14 & 0.00 & 0.00 \\
\hline GDF11 & 226234_PM_at & growth differentiation factor 11 & -1.14 & 0.00 & 0.00 \\
\hline TIMM21 & 223181_PM_at & translocase of inner mitochondrial membra & -1.14 & 0.00 & 0.00 \\
\hline TCF3 & 213811_PM_x_at & transcription factor 3 (E2A immunoglobulin & -1.14 & 0.00 & 0.00 \\
\hline CG030 & 215105_PM_at & uncharacterized CG030 & -1.14 & 0.00 & 0.00 \\
\hline PRKX & 204060_PM_s_at & protein kinase, $\mathrm{X}$-linked & -1.14 & 0.00 & 0.00 \\
\hline ASF1A & 203428_PM_s_at & ASF1 anti-silencing function 1 homolog A ( $S$ & -1.14 & 0.00 & 0.00 \\
\hline--- & 243112_PM_at & --- & -1.14 & 0.00 & 0.00 \\
\hline TNFRSF10A & 241371_PM_at & tumor necrosis factor receptor superfamily, & -1.14 & 0.00 & 0.00 \\
\hline PWP1 & 201606_PM_s_at & PWP1 homolog (S. cerevisiae) & -1.14 & 0.00 & 0.00 \\
\hline UCHL5 & 220083_PM_x_at & ubiquitin carboxyl-terminal hydrolase L5 & -1.14 & 0.00 & 0.00 \\
\hline ZNF783 & 221876_PM_at & zinc finger family member 783 & -1.14 & 0.00 & 0.00 \\
\hline RUFY3 & 227802_PM_at & RUN and FYVE domain containing 3 & -1.14 & 0.00 & 0.00 \\
\hline NEURL & 204889_PM_s_at & neuralized homolog (Drosophila) & -1.14 & 0.00 & 0.00 \\
\hline--- & 214050_PM_at & --- & -1.14 & 0.00 & 0.00 \\
\hline BTBD18 & 215349_PM_at & BTB (POZ) domain containing 18 & -1.14 & 0.00 & 0.00 \\
\hline UHRF1BP1 & 1568789_PM_at & UHRF1 binding protein 1 & -1.14 & 0.00 & 0.00 \\
\hline FKBP4 & 200894_PM_s_at & FK506 binding protein $4,59 \mathrm{kDa}$ & -1.14 & 0.00 & 0.00 \\
\hline BOLA2 & 241644_PM_at & bolA homolog 2 (E. coli) & -1.14 & 0.00 & 0.00 \\
\hline LOC100506282 & 220572_PM_at & uncharacterized LOC100506282 & -1.14 & 0.00 & 0.00 \\
\hline SCAF4 & 222311_PM_s_at & SR-related CTD-associated factor 4 & -1.14 & 0.00 & 0.00 \\
\hline CNP & 208912_PM_s_at & 2',3'-cyclic nucleotide 3' phosphodiesterase & -1.14 & 0.00 & 0.00 \\
\hline GINS3 & 45633_PM_at & GINS complex subunit 3 (Psf3 homolog) & -1.14 & 0.00 & 0.00 \\
\hline--- & 242241_PM_x_at & --- & -1.14 & 0.00 & 0.00 \\
\hline SMARCA4 & 215714_PM_s_at & SWI & -1.14 & 0.00 & 0.00 \\
\hline EIF5B & 201024_PM_x_at & eukaryotic translation initiation factor 5B & -1.14 & 0.00 & 0.00 \\
\hline RPL13 & 214976_PM_at & ribosomal protein L13 & -1.14 & 0.00 & 0.00 \\
\hline NEIL2 & 226585_PM_at & nei endonuclease VIII-like 2 (E. coli) & -1.14 & 0.00 & 0.00 \\
\hline UBE3A & 214980_PM_at & Ubiquitin protein ligase E3A & -1.14 & 0.00 & 0.00 \\
\hline LOC100506453 & 222031_PM_at & uncharacterized LOC100506453 & -1.14 & 0.00 & 0.00 \\
\hline
\end{tabular}




\begin{tabular}{|c|c|c|c|c|c|}
\hline ZC3H14 & 213064_PM_at & zinc finger $\mathrm{CCCH}$-type containing 14 & -1.14 & 0.00 & 0.00 \\
\hline PCYOX1L & 218953_PM_s_at & prenylcysteine oxidase 1 like & -1.14 & 0.00 & 0.00 \\
\hline MTA3 & 223311_PM_s_at & metastasis associated 1 family, member 3 & -1.14 & 0.00 & 0.00 \\
\hline RP9 & 227852_PM_at & retinitis pigmentosa 9 (autosomal dominan & -1.14 & 0.00 & 0.00 \\
\hline URB2 & 205284_PM_at & URB2 ribosome biogenesis 2 homolog (S. c€ & -1.14 & 0.00 & 0.00 \\
\hline--- & 236755_PM_at & --- & -1.14 & 0.00 & 0.00 \\
\hline DUSP8 & 206374_PM_at & dual specificity phosphatase 8 & -1.14 & 0.00 & 0.00 \\
\hline AGAP9 & 242393_PM_x_at & ArfGAP with GTPase domain, ankyrin repea & -1.14 & 0.00 & 0.00 \\
\hline ID3 & 207826_PM_s_at & inhibitor of DNA binding 3 , dominant negati & -1.14 & 0.00 & 0.00 \\
\hline ZNF445 & 229766_PM_at & zinc finger protein 445 & -1.14 & 0.00 & 0.00 \\
\hline--- & 1559119_PM_at & --- & -1.14 & 0.00 & 0.00 \\
\hline POMT1 & 218476_PM_at & protein-O-mannosyltransferase 1 & -1.14 & 0.00 & 0.00 \\
\hline--- & 239165_PM_at & --- & -1.14 & 0.00 & 0.01 \\
\hline PIGU & 225903_PM_at & phosphatidylinositol glycan anchor biosyntr & -1.14 & 0.00 & 0.01 \\
\hline SCAF4 & 222310_PM_at & SR-related CTD-associated factor 4 & -1.14 & 0.00 & 0.01 \\
\hline--- & 242606_PM_at & --- & -1.14 & 0.00 & 0.01 \\
\hline WDR74 & 221712_PM_s_at & WD repeat domain 74 & -1.14 & 0.00 & 0.01 \\
\hline GNPNAT1 & 225853_PM_at & glucosamine-phosphate $\mathrm{N}$-acetyltransferast & -1.14 & 0.00 & 0.01 \\
\hline--- & 241634_PM_at & --- & -1.14 & 0.00 & 0.01 \\
\hline SETDB2 & 238684_PM_at & SET domain, bifurcated 2 & -1.14 & 0.00 & 0.01 \\
\hline FBX041 & 44040_PM_at & F-box protein 41 & -1.14 & 0.00 & 0.01 \\
\hline GAS5 & 227517_PM_s_at & growth arrest-specific 5 (non-protein codin & -1.14 & 0.00 & 0.01 \\
\hline--- & 216440_PM_at & --- & -1.14 & 0.00 & 0.01 \\
\hline UBE2I & 208760_PM_at & ubiquitin-conjugating enzyme E2I & -1.14 & 0.00 & 0.01 \\
\hline METTL2A & 221079_PM_s_at & methyltransferase like $2 \mathrm{~A}$ & -1.14 & 0.00 & 0.01 \\
\hline--- & 226624_PM_at & --- & -1.14 & 0.00 & 0.01 \\
\hline DNAJC27 & 223505_PM_s_at & DnaJ (Hsp40) homolog, subfamily C, membt & -1.14 & 0.00 & 0.01 \\
\hline NUP210 & 220035_PM_at & nucleoporin 210kDa & -1.14 & 0.00 & 0.01 \\
\hline ADCY3 & 209321_PM_s_at & adenylate cyclase 3 & -1.14 & 0.00 & 0.01 \\
\hline NAP1L1 & 208752_PM_x_at & nucleosome assembly protein 1-like 1 & -1.14 & 0.00 & 0.01 \\
\hline--- & 237772_PM_at & --- & -1.14 & 0.00 & 0.01 \\
\hline NIPAL3 & 214579_PM_at & NIPA-like domain containing 3 & -1.14 & 0.00 & 0.01 \\
\hline DNAL1 & 223959_PM_at & dynein, axonemal, light chain 1 & -1.14 & 0.00 & 0.01 \\
\hline
\end{tabular}




\begin{tabular}{|c|c|c|c|c|c|}
\hline--- & 234056_PM_at & --- & -1.14 & 0.00 & 0.01 \\
\hline QDPR & 209123_PM_at & quinoid dihydropteridine reductase & -1.14 & 0.00 & 0.01 \\
\hline RPAIN & 1556088_PM_at & RPA interacting protein & -1.14 & 0.00 & 0.01 \\
\hline TERF1 & 203448_PM_s_at & telomeric repeat binding factor (NIMA-inter & -1.13 & 0.00 & 0.00 \\
\hline RBMX2 & 204097_PM_s_at & RNA binding motif protein, $\mathrm{X}$-linked 2 & -1.13 & 0.00 & 0.00 \\
\hline ARHGEF7 & 202548_PM_s_at & Rho guanine nucleotide exchange factor (G) & -1.13 & 0.00 & 0.00 \\
\hline BANF1 & 210125_PM_s_at & barrier to autointegration factor 1 & -1.13 & 0.00 & 0.00 \\
\hline LOC100271836 & 208118_PM_X_at & smg-1 homolog, phosphatidylinositol 3-kina & -1.13 & 0.00 & 0.00 \\
\hline MYCBP2 & 201960_PM_s_at & MYC binding protein 2, E3 ubiquitin protein & -1.13 & 0.00 & 0.00 \\
\hline ZNF498 & 228138_PM_at & zinc finger protein 498 & -1.13 & 0.00 & 0.00 \\
\hline CCT5 & 208696_PM_at & chaperonin containing TCP1, subunit 5 (eps & -1.13 & 0.00 & 0.00 \\
\hline ATP2A2 & 209186_PM_at & ATPase, Ca++ transporting, cardiac muscle, & -1.13 & 0.00 & 0.00 \\
\hline PTBP1 & 226491_PM_X_at & Polypyrimidine tract binding protein 1 & -1.13 & 0.00 & 0.00 \\
\hline BICD1 & 204741_PM_at & bicaudal D homolog 1 (Drosophila) & -1.13 & 0.00 & 0.00 \\
\hline UTP6 & 218715_PM_at & UTP6, small subunit (SSU) processome com & -1.13 & 0.00 & 0.00 \\
\hline SDK2 & 234073_PM_at & Sidekick cell adhesion molecule 2 & -1.13 & 0.00 & 0.00 \\
\hline CPSF4 & 206688_PM_S_at & cleavage and polyadenylation specific facto & -1.13 & 0.00 & 0.00 \\
\hline AQP11 & 229526_PM_at & aquaporin 11 & -1.13 & 0.00 & 0.00 \\
\hline CTNNB1 & 223679_PM_at & catenin (cadherin-associated protein), beta & -1.13 & 0.00 & 0.00 \\
\hline MON1B & 227116_PM_at & MON1 homolog B (yeast) & -1.13 & 0.00 & 0.00 \\
\hline RBM26 & 222626_PM_at & RNA binding motif protein 26 & -1.13 & 0.00 & 0.00 \\
\hline EP400 & 212375_PM_at & E1A binding protein $\mathrm{p} 400$ & -1.13 & 0.00 & 0.00 \\
\hline ANGEL1 & 36865_PM_at & angel homolog 1 (Drosophila) & -1.13 & 0.00 & 0.00 \\
\hline MRPS5 & 224877_PM_S_at & mitochondrial ribosomal protein $\mathrm{S} 5$ & -1.13 & 0.00 & 0.00 \\
\hline MPRIP & 212197_PM_x_at & myosin phosphatase Rho interacting proteil & -1.13 & 0.00 & 0.00 \\
\hline ERCC1 & 228131_PM_at & excision repair cross-complementing roden & -1.13 & 0.00 & 0.00 \\
\hline CBFA2T2 & 209144_PM_S_at & core-binding factor, runt domain, alpha sub & -1.13 & 0.00 & 0.00 \\
\hline WDR6 & 217734_PM_s_at & WD repeat domain 6 & -1.13 & 0.00 & 0.00 \\
\hline NKTR & 202379_PM_S_at & natural killer-tumor recognition sequence & -1.13 & 0.00 & 0.00 \\
\hline--- & 235531_PM_at & --- & -1.13 & 0.00 & 0.00 \\
\hline CSTF2 & 204459_PM_at & cleavage stimulation factor, 3 ' pre-RNA, suk & -1.13 & 0.00 & 0.00 \\
\hline PPP1R2 & 236754_PM_at & protein phosphatase 1 , regulatory (inhibitol & -1.13 & 0.00 & 0.00 \\
\hline SETD6 & 1554555_PM_a_at & SET domain containing 6 & -1.13 & 0.00 & 0.00 \\
\hline
\end{tabular}




\begin{tabular}{|c|c|c|c|c|c|}
\hline KLHL24 & 221986_PM_s_at & kelch-like 24 (Drosophila) & -1.13 & 0.00 & 0.00 \\
\hline--- & 240478_PM_at & --- & -1.13 & 0.00 & 0.00 \\
\hline GABPB2 & 228466_PM_at & GA binding protein transcription factor, bet & -1.13 & 0.00 & 0.00 \\
\hline--- & 234573_PM_at & --- & -1.13 & 0.00 & 0.00 \\
\hline--- & 238714_PM_at & --- & -1.13 & 0.00 & 0.00 \\
\hline ILKAP & 221548_PM_s_at & integrin-linked kinase-associated serine & -1.13 & 0.00 & 0.00 \\
\hline--- & 238652_PM_at & --- & -1.13 & 0.00 & 0.00 \\
\hline--- & 239969_PM_at & --- & -1.13 & 0.00 & 0.00 \\
\hline--- & 242968_PM_at & --- & -1.13 & 0.00 & 0.00 \\
\hline--- & 232788_PM_at & --- & -1.13 & 0.00 & 0.00 \\
\hline SRSF4 & 242837_PM_at & Serine & -1.13 & 0.00 & 0.00 \\
\hline APOBEC3G & 215579_PM_at & apolipoprotein B mRNA editing enzyme, cat & -1.13 & 0.00 & 0.00 \\
\hline--- & 243414_PM_at & --- & -1.13 & 0.00 & 0.00 \\
\hline--- & 228615_PM_at & --- & -1.13 & 0.00 & 0.00 \\
\hline AP1G2 & 214341_PM_at & adaptor-related protein complex 1, gamma & -1.13 & 0.00 & 0.00 \\
\hline MLL & 226981_PM_at & myeloid & -1.13 & 0.00 & 0.00 \\
\hline AARSD1 & 222064_PM_s_at & alanyl-tRNA synthetase domain containing & -1.13 & 0.00 & 0.00 \\
\hline HLA-DRB1 & 209312_PM_x_at & major histocompatibility complex, class II, [ & -1.13 & 0.00 & 0.00 \\
\hline DIS3L2 & 227597_PM_at & DIS3 mitotic control homolog (S. cerevisiae) & -1.13 & 0.00 & 0.00 \\
\hline ATXN7 & 204516_PM_at & ataxin 7 & -1.13 & 0.00 & 0.00 \\
\hline--- & 241373_PM_at & --- & -1.13 & 0.00 & 0.00 \\
\hline DDX52 & 213637_PM_at & DEAD (Asp-Glu-Ala-Asp) box polypeptide 52 & -1.13 & 0.00 & 0.00 \\
\hline--- & 222282_PM_at & --- & -1.13 & 0.00 & 0.00 \\
\hline GPM6B & 209170_PM_s_at & glycoprotein M6B & -1.13 & 0.00 & 0.00 \\
\hline GRSF1 & 221917_PM_s_at & G-rich RNA sequence binding factor 1 & -1.13 & 0.00 & 0.00 \\
\hline PPP1R13B & 216347_PM_s_at & protein phosphatase 1 , regulatory subunit 1 & -1.13 & 0.00 & 0.00 \\
\hline COPS7B & 225696_PM_at & COP9 constitutive photomorphogenic homc & -1.13 & 0.00 & 0.00 \\
\hline CCNJ & 219470_PM_x_at & cyclin J & -1.13 & 0.00 & 0.00 \\
\hline RPRML & 1554655_PM_a_at & reprimo-like & -1.13 & 0.00 & 0.00 \\
\hline FBXO21 & 214891_PM_at & F-box protein 21 & -1.13 & 0.00 & 0.00 \\
\hline KIAA0947 & 209654_PM_at & KIAA0947 & -1.13 & 0.00 & 0.00 \\
\hline SLC7A6OS & 232057_PM_at & solute carrier family 7 , member 6 opposite : & -1.13 & 0.00 & 0.00 \\
\hline DAAM1 & 232552_PM_at & dishevelled associated activator of morpho & -1.13 & 0.00 & 0.00 \\
\hline
\end{tabular}




\begin{tabular}{|c|c|c|c|c|c|}
\hline--- & 239619_PM_at & --- & -1.13 & 0.00 & 0.01 \\
\hline $\mathrm{ZADH} 2$ & 243417_PM_at & zinc binding alcohol dehydrogenase domair & -1.13 & 0.00 & 0.01 \\
\hline FARP1 & 201910_PM_at & FERM, RhoGEF (ARHGEF) and pleckstrin dor & -1.13 & 0.00 & 0.01 \\
\hline RASA3 & 225562_PM_at & RAS p21 protein activator 3 & -1.13 & 0.00 & 0.01 \\
\hline ZCCHC3 & 225072_PM_at & zinc finger, $\mathrm{CCHC}$ domain containing 3 & -1.13 & 0.00 & 0.01 \\
\hline THOC1 & 204064_PM_at & THO complex 1 & -1.13 & 0.00 & 0.01 \\
\hline SLC7A6 & 203579_PM_s_at & solute carrier family 7 (amino acid transpor & -1.13 & 0.00 & 0.01 \\
\hline--- & 240899_PM_at & --- & -1.13 & 0.00 & 0.01 \\
\hline PLA2G12A & 242323_PM_at & phospholipase $A 2$, group XIIA & -1.13 & 0.00 & 0.01 \\
\hline ADO & 212502_PM_at & 2-aminoethanethiol (cysteamine) dioxygen: & -1.13 & 0.00 & 0.01 \\
\hline RWDD2B & 218377_PM_s_at & RWD domain containing $2 \mathrm{~B}$ & -1.13 & 0.00 & 0.01 \\
\hline--- & 1560209_PM_at & --- & -1.13 & 0.00 & 0.01 \\
\hline MDN1 & 212693_PM_at & MDN1, midasin homolog (yeast) & -1.13 & 0.00 & 0.01 \\
\hline HERC2P7 & 232900_PM_at & hect domain and RLD 2 pseudogene 7 & -1.13 & 0.00 & 0.01 \\
\hline YLPM1 & 212787_PM_at & YLP motif containing 1 & -1.13 & 0.00 & 0.01 \\
\hline HSP90B1 & 216449_PM_x_at & heat shock protein 90kDa beta (Grp94), me & -1.13 & 0.00 & 0.01 \\
\hline LPHN1 & 219145_PM_at & latrophilin 1 & -1.13 & 0.00 & 0.01 \\
\hline HLA-DRB1 & 215193_PM_x_at & major histocompatibility complex, class II, [ & -1.13 & 0.00 & 0.01 \\
\hline HUWE1 & 208599_PM_at & HECT, UBA and WWE domain containing 1 , & -1.13 & 0.00 & 0.01 \\
\hline TRAPPC10 & 215269_PM_at & trafficking protein particle complex 10 & -1.13 & 0.00 & 0.01 \\
\hline PDSS1 & 236298_PM_at & Prenyl (decaprenyl) diphosphate synthase, : & -1.13 & 0.00 & 0.01 \\
\hline LYRM7 & 240344_PM_x_at & Lyrm7 homolog (mouse) & -1.13 & 0.00 & 0.01 \\
\hline SMAD3 & 218284_PM_at & SMAD family member 3 & -1.13 & 0.00 & 0.01 \\
\hline CBX5 & 209715_PM_at & chromobox homolog 5 & -1.13 & 0.00 & 0.01 \\
\hline LOC157562 & 213776_PM_at & uncharacterized LOC157562 & -1.13 & 0.00 & 0.01 \\
\hline TAF2 & 1554721_PM_a_at & TAF2 RNA polymerase II, TATA box binding & -1.13 & 0.00 & 0.01 \\
\hline DERL2 & 226719_PM_at & derlin 2 & -1.12 & 0.00 & 0.00 \\
\hline TBP & 203135_PM_at & TATA box binding protein & -1.12 & 0.00 & 0.00 \\
\hline ZNF562 & 219163_PM_at & zinc finger protein 562 & -1.12 & 0.00 & 0.00 \\
\hline SFPQ & 214016_PM_s_at & splicing factor proline & -1.12 & 0.00 & 0.00 \\
\hline ZNF212 & 203985_PM_at & zinc finger protein 212 & -1.12 & 0.00 & 0.00 \\
\hline ATG16L1 & 232612_PM_s_at & autophagy related 16 -like 1 (S. cerevisiae) & -1.12 & 0.00 & 0.00 \\
\hline SMU1 & 222618_PM_at & smu-1 suppressor of mec- 8 and unc- 52 hom & -1.12 & 0.00 & 0.00 \\
\hline
\end{tabular}




\begin{tabular}{|c|c|c|c|c|c|}
\hline DVL2 & 57532_PM_at & dishevelled, dsh homolog 2 (Drosophila) & -1.12 & 0.00 & 0.00 \\
\hline RAB3GAP1 & 213531_PM_s_at & RAB3 GTPase activating protein subunit 1 (c & -1.12 & 0.00 & 0.00 \\
\hline--- & 235862_PM_at & --- & -1.12 & 0.00 & 0.00 \\
\hline DDX27 & 221780_PM_s_at & DEAD (Asp-Glu-Ala-Asp) box polypeptide 27 & -1.12 & 0.00 & 0.00 \\
\hline RNF115 & 212742_PM_at & ring finger protein 115 & -1.12 & 0.00 & 0.00 \\
\hline SBF1 & 213383_PM_at & SET binding factor 1 & -1.12 & 0.00 & 0.00 \\
\hline KDM2B & 226215_PM_s_at & lysine $(K)$-specific demethylase $2 \mathrm{~B}$ & -1.12 & 0.00 & 0.00 \\
\hline HSPG2 & 201655_PM_s_at & heparan sulfate proteoglycan 2 & -1.12 & 0.00 & 0.00 \\
\hline GALNT11 & 219013_PM_at & UDP-N-acetyl-alpha-D-galactosamine:polyp & -1.12 & 0.00 & 0.00 \\
\hline HN1L & 212115_PM_at & hematological and neurological expressed 1 & -1.12 & 0.00 & 0.00 \\
\hline DOLPP1 & 221817_PM_at & dolichyl pyrophosphate phosphatase 1 & -1.12 & 0.00 & 0.00 \\
\hline HNRPDL & 209067_PM_s_at & heterogeneous nuclear ribonucleoprotein [ & -1.12 & 0.00 & 0.00 \\
\hline RPL18A & 200869_PM_at & ribosomal protein L18a & -1.12 & 0.00 & 0.00 \\
\hline PPP6R2 & 202792_PM_s_at & protein phosphatase 6 , regulatory subunit $\bar{z}$ & -1.12 & 0.00 & 0.00 \\
\hline RNMT & 202683_PM_s_at & RNA (guanine-7-) methyltransferase & -1.12 & 0.00 & 0.00 \\
\hline ZNF747 & 228856_PM_at & zinc finger protein 747 & -1.12 & 0.00 & 0.00 \\
\hline YPEL1 & 228788_PM_at & yippee-like 1 (Drosophila) & -1.12 & 0.00 & 0.00 \\
\hline NUCKS1 & 217802_PM_s_at & nuclear casein kinase and cyclin-dependent & -1.12 & 0.00 & 0.00 \\
\hline MRPS27 & 212145_PM_at & mitochondrial ribosomal protein S27 & -1.12 & 0.00 & 0.00 \\
\hline DNAJC16 & 212911_PM_at & DnaJ (Hsp40) homolog, subfamily C, membe & -1.12 & 0.00 & 0.00 \\
\hline MTMR9 & 204837_PM_at & myotubularin related protein 9 & -1.12 & 0.00 & 0.00 \\
\hline PPP2R1B & 202883_PM_s_at & protein phosphatase 2 , regulatory subunit 1 & -1.12 & 0.00 & 0.00 \\
\hline LETM1 & 222006_PM_at & leucine zipper-EF-hand containing transmer & -1.12 & 0.00 & 0.00 \\
\hline AMMECR1 & 226421_PM_at & Alport syndrome, mental retardation, midf $\bar{c}$ & -1.12 & 0.00 & 0.00 \\
\hline GOSR1 & 204630_PM_s_at & golgi SNAP receptor complex member 1 & -1.12 & 0.00 & 0.00 \\
\hline MPRIP & 214771_PM_x_at & myosin phosphatase Rho interacting proteiı & -1.12 & 0.00 & 0.00 \\
\hline NHP2L1 & 201076_PM_at & NHP2 non-histone chromosome protein $2-\mathrm{li}$ & -1.12 & 0.00 & 0.00 \\
\hline POLR3A & 231763_PM_at & polymerase (RNA) III (DNA directed) polype & -1.12 & 0.00 & 0.00 \\
\hline LSM4 & 212924_PM_s_at & LSM4 homolog, U6 small nuclear RNA assoc & -1.12 & 0.00 & 0.00 \\
\hline KCMF1 & 242887_PM_at & potassium channel modulatory factor 1 & -1.12 & 0.00 & 0.00 \\
\hline--- & 241448_PM_at & --- & -1.12 & 0.00 & 0.00 \\
\hline PCBP1-AS1 & 235482_PM_at & PCBP1 antisense RNA 1 (non-protein coding & -1.12 & 0.00 & 0.00 \\
\hline CDC23 & 202892_PM_at & cell division cycle 23 homolog (S. cerevisiae & -1.12 & 0.00 & 0.00 \\
\hline
\end{tabular}




\begin{tabular}{|c|c|c|c|c|c|}
\hline GTPBP8 & 221046_PM_s_at & GTP-binding protein 8 (putative) & -1.12 & 0.00 & 0.00 \\
\hline LUC7L & 223295_PM_s_at & LUC7-like (S. cerevisiae) & -1.12 & 0.00 & 0.00 \\
\hline RCL1 & 222666_PM_s_at & RNA terminal phosphate cyclase-like 1 & -1.12 & 0.00 & 0.00 \\
\hline TXNDC15 & 220495_PM_s_at & thioredoxin domain containing 15 & -1.12 & 0.00 & 0.00 \\
\hline FBXW7 & 218751_PM_s_at & F-box and WD repeat domain containing 7 , & -1.12 & 0.00 & 0.00 \\
\hline--- & 237914_PM_s_at & --- & -1.12 & 0.00 & 0.00 \\
\hline GCFC1-AS1 & 239407_PM_at & GCFC1 antisense RNA 1 (non-protein codin€ & -1.12 & 0.00 & 0.00 \\
\hline SNRNP27 & 212438_PM_at & small nuclear ribonucleoprotein 27kDa (U4 & -1.12 & 0.00 & 0.00 \\
\hline TAF15 & 227891_PM_s_at & TAF15 RNA polymerase II, TATA box binding & -1.12 & 0.00 & 0.00 \\
\hline ELP3 & 227075_PM_at & elongation protein 3 homolog (S. cerevisiae & -1.12 & 0.00 & 0.01 \\
\hline LIN7C & 223350_PM_x_at & lin-7 homolog C (C. elegans) & -1.12 & 0.00 & 0.01 \\
\hline ZNF789 & 235231_PM_at & zinc finger protein 789 & -1.12 & 0.00 & 0.01 \\
\hline--- & 241301_PM_at & --- & -1.12 & 0.00 & 0.01 \\
\hline--- & 243498_PM_at & --- & -1.12 & 0.00 & 0.01 \\
\hline PTPMT1 & 225901_PM_at & protein tyrosine phosphatase, mitochondric & -1.12 & 0.00 & 0.01 \\
\hline ZBTB4 & 227047_PM_x_at & zinc finger and BTB domain containing 4 & -1.12 & 0.00 & 0.01 \\
\hline SLC16A1 & 209900_PM_s_at & solute carrier family 16 , member 1 (monocí & -1.12 & 0.00 & 0.01 \\
\hline ACSS1 & 224882_PM_at & acyl-CoA synthetase short-chain family mer & -1.12 & 0.00 & 0.01 \\
\hline HIP1R & 209559_PM_at & huntingtin interacting protein 1 related & -1.12 & 0.00 & 0.01 \\
\hline TNFRSF10A & 231775_PM_at & tumor necrosis factor receptor superfamily, & -1.12 & 0.00 & 0.01 \\
\hline--- & 244648_PM_at & --- & -1.12 & 0.00 & 0.01 \\
\hline LOC100507602 & 1557507_PM_at & uncharacterized LOC100507602 & -1.12 & 0.00 & 0.01 \\
\hline MTA1 & 211783_PM_s_at & metastasis associated 1 & -1.12 & 0.00 & 0.01 \\
\hline EXOSC7 & 212627_PM_s_at & exosome component 7 & -1.12 & 0.00 & 0.01 \\
\hline CCL20 & 205476_PM_at & chemokine ( $\mathrm{C}-\mathrm{C}$ motif) ligand 20 & -1.12 & 0.00 & 0.01 \\
\hline DIEXF & 204699_PM_s_at & digestive organ expansion factor homolog (: & -1.12 & 0.00 & 0.01 \\
\hline UBE2G2 & 209042_PM_s_at & ubiquitin-conjugating enzyme E2G 2 & -1.12 & 0.00 & 0.01 \\
\hline RBM4 & 235511_PM_at & RNA binding motif protein 4 & -1.12 & 0.00 & 0.01 \\
\hline LRCH3 & 1559490_PM_at & leucine-rich repeats and calponin homology & -1.12 & 0.00 & 0.01 \\
\hline--- & 220969_PM_s_at & --- & -1.12 & 0.00 & 0.01 \\
\hline TGFBRAP1 & 221235_PM_s_at & transforming growth factor, beta receptor $\bar{c}$ & -1.12 & 0.00 & 0.01 \\
\hline HNRNPH3 & 210588_PM_x_at & heterogeneous nuclear ribonucleoprotein $\vdash$ & -1.12 & 0.00 & 0.01 \\
\hline NONO & 208698_PM_s_at & non-POU domain containing, octamer-bindi & -1.11 & 0.00 & 0.00 \\
\hline
\end{tabular}




\begin{tabular}{|c|c|c|c|c|c|}
\hline SRSF5 & 212266_PM_s_at & serine & -1.11 & 0.00 & 0.00 \\
\hline GTF2H1 & 202451_PM_at & general transcription factor $\mathrm{IIH}$, polypeptid $\epsilon$ & -1.11 & 0.00 & 0.00 \\
\hline GLS & 203159_PM_at & glutaminase & -1.11 & 0.00 & 0.00 \\
\hline SLC30A7 & 226217_PM_at & solute carrier family 30 (zinc transporter), $n$ & -1.11 & 0.00 & 0.00 \\
\hline KIAA0907 & 202220_PM_at & KIAA0907 & -1.11 & 0.00 & 0.00 \\
\hline cox18 & 230909_PM_at & COX18 cytochrome c oxidase assembly hor & -1.11 & 0.00 & 0.00 \\
\hline HNRNPA2B1 & 225932_PM_s_at & heterogeneous nuclear ribonucleoprotein $A$ & -1.11 & 0.00 & 0.00 \\
\hline--- & 228638_PM_at & --- & -1.11 & 0.00 & 0.00 \\
\hline NCOA5 & 225145_PM_at & nuclear receptor coactivator 5 & -1.11 & 0.00 & 0.00 \\
\hline FANCE & 220255_PM_at & Fanconi anemia, complementation group $\mathrm{E}$ & -1.11 & 0.00 & 0.00 \\
\hline FBXW11 & 209455_PM_at & F-box and WD repeat domain containing 11 & -1.11 & 0.00 & 0.00 \\
\hline THEM6 & 218500_PM_at & thioesterase superfamily member 6 & -1.11 & 0.00 & 0.00 \\
\hline USP33 & 214843_PM_s_at & ubiquitin specific peptidase 33 & -1.11 & 0.00 & 0.00 \\
\hline--- & 240217_PM_s_at & --- & -1.11 & 0.00 & 0.00 \\
\hline HNRNPL & 221860_PM_at & heterogeneous nuclear ribonucleoprotein $\mathrm{L}$ & -1.11 & 0.00 & 0.00 \\
\hline CHRAC1 & 231764_PM_at & chromatin accessibility complex 1 & -1.11 & 0.00 & 0.00 \\
\hline UBE2Z & 222395_PM_s_at & ubiquitin-conjugating enzyme E2Z & -1.11 & 0.00 & 0.00 \\
\hline SDHAP1 & 226693_PM_at & Succinate dehydrogenase complex, subunit & -1.11 & 0.00 & 0.00 \\
\hline HNRNPR & 208766_PM_s_at & heterogeneous nuclear ribonucleoprotein $\mathrm{R}$ & -1.11 & 0.00 & 0.00 \\
\hline SLC25A12 & 203340_PM_s_at & solute carrier family 25 (aspartate & -1.11 & 0.00 & 0.00 \\
\hline--- & 240128_PM_at & --- & -1.11 & 0.00 & 0.00 \\
\hline FNBP1 & 230389_PM_at & formin binding protein 1 & -1.11 & 0.00 & 0.00 \\
\hline RRP9 & 204133_PM_at & ribosomal RNA processing 9, small subunit ( & -1.11 & 0.00 & 0.01 \\
\hline NSUN2 & 223076_PM_s_at & NOP2 & -1.11 & 0.00 & 0.01 \\
\hline FBRSL1 & 225704_PM_at & fibrosin-like 1 & -1.11 & 0.00 & 0.01 \\
\hline PSMD11 & 208776_PM_at & proteasome (prosome, macropain) 26S sub & -1.11 & 0.00 & 0.01 \\
\hline KCTD10 & 226518_PM_at & potassium channel tetramerisation domain & -1.11 & 0.00 & 0.01 \\
\hline--- & 217554_PM_at & --- & -1.11 & 0.00 & 0.01 \\
\hline ZBTB20 & 235308_PM_at & zinc finger and BTB domain containing 20 & -1.11 & 0.00 & 0.01 \\
\hline NFATC1 & 208196_PM_x_at & nuclear factor of activated T-cells, cytoplasr & -1.11 & 0.00 & 0.01 \\
\hline RREB1 & 242297_PM_at & ras responsive element binding protein 1 & -1.11 & 0.00 & 0.01 \\
\hline AKIRIN1 & 222458_PM_s_at & akirin 1 & -1.11 & 0.00 & 0.01 \\
\hline CSNK2A1 & 212073_PM_at & casein kinase 2 , alpha 1 polypeptide & -1.11 & 0.00 & 0.01 \\
\hline
\end{tabular}




\begin{tabular}{|c|c|c|c|c|c|}
\hline COPB2 & 226256_PM_at & coatomer protein complex, subunit beta 2 ( & -1.11 & 0.00 & 0.01 \\
\hline EPM2AIP1 & 236314_PM_at & EPM2A (laforin) interacting protein 1 & -1.11 & 0.00 & 0.01 \\
\hline THAP6 & 230169_PM_at & THAP domain containing 6 & -1.11 & 0.00 & 0.01 \\
\hline GIMAP6 & 219777_PM_at & GTPase, IMAP family member 6 & -1.11 & 0.00 & 0.01 \\
\hline--- & 238038_PM_at & --- & -1.11 & 0.00 & 0.01 \\
\hline IL12RB1 & 1552584_PM_at & interleukin 12 receptor, beta 1 & -1.11 & 0.00 & 0.01 \\
\hline AKR1B1 & 201272_PM_at & aldo-keto reductase family 1 , member B1 ( $\bar{c}$ & -1.11 & 0.00 & 0.01 \\
\hline HNRNPD & 221481_PM_x_at & heterogeneous nuclear ribonucleoprotein [ & -1.1 & 0.00 & 0.00 \\
\hline FAM192A & 224248_PM_x_at & family with sequence similarity 192, memb & -1.1 & 0.00 & 0.00 \\
\hline MTMR1 & 216095_PM_x_at & myotubularin related protein 1 & -1.1 & 0.00 & 0.00 \\
\hline SBNO1 & 230407_PM_at & strawberry notch homolog 1 (Drosophila) & -1.1 & 0.00 & 0.00 \\
\hline TARDBP & 200020_PM_at & TAR DNA binding protein & -1.1 & 0.00 & 0.00 \\
\hline LEPROTL1 & 202595_PM_s_at & leptin receptor overlapping transcript-like 1 & -1.1 & 0.00 & 0.00 \\
\hline APH1A & 218389_PM_s_at & anterior pharynx defective 1 homolog A (C. & -1.1 & 0.00 & 0.00 \\
\hline WIPI2 & 202031_PM_s_at & WD repeat domain, phosphoinositide interc & -1.1 & 0.00 & 0.00 \\
\hline MTMR1 & 213511_PM_s_at & myotubularin related protein 1 & -1.1 & 0.00 & 0.00 \\
\hline ZBTB43 & 231393_PM_x_at & Zinc finger and BTB domain containing 43 & -1.1 & 0.00 & 0.00 \\
\hline HEATR5B & 233642_PM_s_at & HEAT repeat containing $5 \mathrm{~B}$ & -1.1 & 0.00 & 0.00 \\
\hline RPL22 & 214042_PM_s_at & ribosomal protein L22 & -1.1 & 0.00 & 0.00 \\
\hline RARRES1 & 206391_PM_at & retinoic acid receptor responder (tazaroten & -1.1 & 0.00 & 0.00 \\
\hline PTBP1 & 211270_PM_x_at & polypyrimidine tract binding protein 1 & -1.1 & 0.00 & 0.00 \\
\hline LSM2 & 209449_PM_at & LSM2 homolog, U6 small nuclear RNA assoc & -1.1 & 0.00 & 0.00 \\
\hline TM2D2 & 224413_PM_s_at & TM2 domain containing 2 & -1.1 & 0.00 & 0.00 \\
\hline CRYZL1 & 219767_PM_s_at & crystallin, zeta (quinone reductase)-like 1 & -1.1 & 0.00 & 0.00 \\
\hline SLC35A2 & 209326_PM_at & solute carrier family 35 (UDP-galactose tran & -1.1 & 0.00 & 0.00 \\
\hline NAB2 & 212803_PM_at & NGFI-A binding protein 2 (EGR1 binding pro & -1.1 & 0.00 & 0.00 \\
\hline PABPC1L & 233104_PM_at & poly(A) binding protein, cytoplasmic 1-like & -1.1 & 0.00 & 0.00 \\
\hline SON & 226465_PM_s_at & SON DNA binding protein & -1.1 & 0.00 & 0.00 \\
\hline RPL28 & 200003_PM_s_at & ribosomal protein L28 & -1.1 & 0.00 & 0.00 \\
\hline INO80 & 229356_PM_x_at & INO80 homolog (S. cerevisiae) & -1.1 & 0.00 & 0.00 \\
\hline--- & 222316_PM_at & --- & -1.1 & 0.00 & 0.01 \\
\hline ARMC8 & 1555281_PM_x_at & armadillo repeat containing 8 & -1.1 & 0.00 & 0.01 \\
\hline$A B C C 1$ & 202805_PM_s_at & ATP-binding cassette, sub-family C (CFTR & -1.1 & 0.00 & 0.01 \\
\hline
\end{tabular}




\begin{tabular}{|c|c|c|c|c|c|}
\hline SLC25A25 & 225212_PM_at & solute carrier family 25 (mitochondrial carri & -1.1 & 0.00 & 0.01 \\
\hline PDE6D & 216883_PM_x_at & phosphodiesterase 6D, cGMP-specific, rod, & -1.1 & 0.00 & 0.01 \\
\hline SRSF2 & 214882_PM_s_at & serine & -1.1 & 0.00 & 0.01 \\
\hline SMPD1 & 217171_PM_at & sphingomyelin phosphodiesterase 1 , acid ly & -1.1 & 0.00 & 0.01 \\
\hline ATXN2 & 202622_PM_s_at & ataxin 2 & -1.1 & 0.00 & 0.01 \\
\hline SEC63 & 229969_PM_at & SEC63 homolog (S. cerevisiae) & -1.1 & 0.00 & 0.01 \\
\hline RARG & 204189_PM_at & retinoic acid receptor, gamma & -1.1 & 0.00 & 0.01 \\
\hline EPHA2 & 203499_PM_at & EPH receptor $A 2$ & -1.1 & 0.00 & 0.01 \\
\hline--- & 227052_PM_at & --- & -1.1 & 0.00 & 0.01 \\
\hline NUDCD3 & 201270_PM_x_at & NudC domain containing 3 & -1.1 & 0.00 & 0.01 \\
\hline PDE8A & 212521_PM_s_at & phosphodiesterase 8A & -1.1 & 0.00 & 0.01 \\
\hline PRPF38A & 223230_PM_at & PRP38 pre-mRNA processing factor 38 (yea؛ & -1.1 & 0.00 & 0.01 \\
\hline LARP7 & 241385_PM_at & La ribonucleoprotein domain family, memb & -1.1 & 0.00 & 0.01 \\
\hline WDR77 & 201421_PM_s_at & WD repeat domain 77 & -1.1 & 0.00 & 0.01 \\
\hline C20orf11 & 218448_PM_at & chromosome 20 open reading frame 11 & -1.1 & 0.00 & 0.01 \\
\hline RABL2A & 220500_PM_s_at & RAB, member of RAS oncogene family-like $z$ & -1.1 & 0.00 & 0.01 \\
\hline THOC2 & 222122_PM_s_at & THO complex 2 & -1.1 & 0.00 & 0.01 \\
\hline BLCAP & 201032_PM_at & bladder cancer associated protein & -1.09 & 0.00 & 0.00 \\
\hline STK35 & 225649_PM_s_at & serine & -1.09 & 0.00 & 0.00 \\
\hline SLC35E1 & 227518_PM_at & solute carrier family 35 , member E1 & -1.09 & 0.00 & 0.00 \\
\hline--- & 242233_PM_at & --- & -1.09 & 0.00 & 0.00 \\
\hline ELK1 & 203617_PM_x_at & ELK1, member of ETS oncogene family & -1.09 & 0.00 & 0.00 \\
\hline LSM14A & 212132_PM_at & LSM14A, SCD6 homolog A (S. cerevisiae) & -1.09 & 0.00 & 0.00 \\
\hline FGD2 & 1553906_PM_s_at & FYVE, RhoGEF and PH domain containing 2 & -1.09 & 0.00 & 0.00 \\
\hline--- & 1554597_PM_at & --- & -1.09 & 0.00 & 0.00 \\
\hline TMF1 & 214948_PM_s_at & TATA element modulatory factor 1 & -1.09 & 0.00 & 0.00 \\
\hline ECD & 203013_PM_at & ecdysoneless homolog (Drosophila) & -1.09 & 0.00 & 0.00 \\
\hline QRICH1 & 209174_PM_s_at & glutamine-rich 1 & -1.09 & 0.00 & 0.00 \\
\hline DAZAP1 & 226620_PM_x_at & DAZ associated protein 1 & -1.09 & 0.00 & 0.00 \\
\hline SRSF2 & 200754_PM_x_at & serine & -1.09 & 0.00 & 0.00 \\
\hline ANKRD11 & 238538_PM_at & ankyrin repeat domain 11 & -1.09 & 0.00 & 0.00 \\
\hline GTF2H3 & 1554599_PM_x_at & general transcription factor IIH, polypeptid $\epsilon$ & -1.09 & 0.00 & 0.00 \\
\hline AAGAB & 202851_PM_at & alpha- and gamma-adaptin binding protein & -1.09 & 0.00 & 0.01 \\
\hline
\end{tabular}




\begin{tabular}{|c|c|c|c|c|c|}
\hline FOXP1 & 224838_PM_at & forkhead box P1 & -1.09 & 0.00 & 0.01 \\
\hline SUGP2 & 214092_PM_x_at & SURP and $\mathrm{G}$ patch domain containing 2 & -1.09 & 0.00 & 0.01 \\
\hline POLR2D & 203664_PM_s_at & polymerase (RNA) II (DNA directed) polyper & -1.09 & 0.00 & 0.01 \\
\hline UBE3B & 212403_PM_at & ubiquitin protein ligase E3B & -1.09 & 0.00 & 0.01 \\
\hline MAР3К13 & 206249_PM_at & mitogen-activated protein kinase kinase kin & -1.09 & 0.00 & 0.01 \\
\hline--- & 214949_PM_at & --- & -1.09 & 0.00 & 0.01 \\
\hline TMEM97 & 214283_PM_at & transmembrane protein 97 & -1.09 & 0.00 & 0.01 \\
\hline TCF20 & 212931_PM_at & transcription factor 20 (AR1) & -1.09 & 0.00 & 0.01 \\
\hline RBM48 & 221595_PM_at & RNA binding motif protein 48 & -1.09 & 0.00 & 0.01 \\
\hline UTP23 & 227402_PM_s_at & UTP23, small subunit (SSU) processome cor & -1.09 & 0.00 & 0.01 \\
\hline FUS & 200959_PM_at & fused in sarcoma & -1.09 & 0.00 & 0.01 \\
\hline IFT20 & 210312_PM_s_at & intraflagellar transport 20 homolog (Chlam) & -1.09 & 0.00 & 0.01 \\
\hline USP7 & 201498_PM_at & ubiquitin specific peptidase 7 (herpes virus- & -1.09 & 0.00 & 0.01 \\
\hline TSPYL1 & 221493_PM_at & TSPY-like 1 & -1.09 & 0.00 & 0.01 \\
\hline HMGN1 & 200943_PM_at & high mobility group nucleosome binding do & -1.09 & 0.00 & 0.01 \\
\hline SRSF1 & 211784_PM_s_at & serine & -1.08 & 0.00 & 0.00 \\
\hline R3HDM2 & 203831_PM_at & R3H domain containing 2 & -1.08 & 0.00 & 0.00 \\
\hline BBIP1 & 228993_PM_s_at & BBSome interacting protein 1 & -1.08 & 0.00 & 0.00 \\
\hline 6V1G2-DDX39B & 200041_PM_s_at & ATP6V1G2-DDX39B readthrough (non-prote & -1.08 & 0.00 & 0.00 \\
\hline FAM192A & 223406_PM_x_at & family with sequence similarity 192, memb & -1.08 & 0.00 & 0.00 \\
\hline NONO & 210470_PM_x_at & non-POU domain containing, octamer-bindi & -1.08 & 0.00 & 0.00 \\
\hline RPLP2 & 200909_PM_s_at & ribosomal protein, large, P2 & -1.08 & 0.00 & 0.00 \\
\hline MAPK1IP1L & 212643_PM_at & mitogen-activated protein kinase 1 interact & -1.08 & 0.00 & 0.00 \\
\hline LRRC47 & 212904_PM_at & leucine rich repeat containing 47 & -1.08 & 0.00 & 0.01 \\
\hline PTDSS1 & 201433_PM_s_at & phosphatidylserine synthase 1 & -1.08 & 0.00 & 0.01 \\
\hline CCNK & 225824_PM_at & cyclin K & -1.08 & 0.00 & 0.01 \\
\hline LGALS8 & 208935_PM_S_at & lectin, galactoside-binding, soluble, 8 & -1.08 & 0.00 & 0.01 \\
\hline NUB1 & 222512_PM_at & negative regulator of ubiquitin-like proteins & -1.08 & 0.00 & 0.01 \\
\hline FAM108B1 & 227551_PM_at & family with sequence similarity 108, memb & -1.08 & 0.00 & 0.01 \\
\hline NEK9 & 212299_PM_at & NIMA (never in mitosis gene a)- related kina & -1.08 & 0.00 & 0.01 \\
\hline SECISBP2 & 218265_PM_at & SECIS binding protein 2 & -1.08 & 0.00 & 0.01 \\
\hline MIR1304 & 222728_PM_s_at & microRNA 1304 & -1.08 & 0.00 & 0.01 \\
\hline CALM1 & 200623_PM_s_at & calmodulin 1 (phosphorylase kinase, delta) & -1.08 & 0.00 & 0.01 \\
\hline
\end{tabular}




\begin{tabular}{|c|c|c|c|c|c|}
\hline NDFIP1 & 222422_PM_s_at & Nedd 4 family interacting protein 1 & -1.08 & 0.00 & 0.01 \\
\hline EPC1 & 234969_PM_s_at & enhancer of polycomb homolog 1 (Drosoph & -1.08 & 0.00 & 0.01 \\
\hline METTL3 & 209265_PM_s_at & methyltransferase like 3 & -1.08 & 0.00 & 0.01 \\
\hline SNW1 & 201575_PM_at & SNW domain containing 1 & -1.08 & 0.00 & 0.01 \\
\hline CNOT2 & 222182_PM_s_at & CCR4-NOT transcription complex, subunit 2 & -1.08 & 0.00 & 0.01 \\
\hline NONO & 200057_PM_s_at & non-POU domain containing, octamer-bindi & -1.07 & 0.00 & 0.00 \\
\hline CELF1 & 221743_PM_at & CUGBP, Elav-like family member 1 & -1.07 & 0.00 & 0.00 \\
\hline YTHDF1 & 221741_PM_s_at & YTH domain family, member 1 & -1.07 & 0.00 & 0.00 \\
\hline FDFT1 & 208647_PM_at & farnesyl-diphosphate farnesyltransferase 1 & -1.07 & 0.00 & 0.01 \\
\hline ALKBH5 & 234302_PM_s_at & alkB, alkylation repair homolog 5 (E. coli) & -1.07 & 0.00 & 0.01 \\
\hline SUDS3 & 224974_PM_at & suppressor of defective silencing 3 homolo६ & -1.07 & 0.00 & 0.01 \\
\hline OSBP & 201800_PM_s_at & oxysterol binding protein & -1.07 & 0.00 & 0.01 \\
\hline LOC100505877 & 239880_PM_at & uncharacterized LOC100505877 & -1.07 & 0.00 & 0.02 \\
\hline SRRM1 & 201225_PM_s_at & serine & -1.07 & 0.00 & 0.02 \\
\hline GLE1 & 225371_PM_at & GLE1 RNA export mediator homolog (yeast) & -1.06 & 0.00 & 0.01 \\
\hline PITPNB & 202522_PM_at & phosphatidylinositol transfer protein, beta & -1.06 & 0.00 & 0.02 \\
\hline KCMF1 & 217938_PM_s_at & potassium channel modulatory factor 1 & 1.07 & 0.00 & 0.0 \\
\hline--- & 1566881_PM_at & --- & 1.07 & 0.00 & 0.01 \\
\hline SELL & 204563_PM_at & selectin L & 1.07 & 0.00 & 0.01 \\
\hline DDAH1 & 243711_PM_at & dimethylarginine dimethylaminohydrolase & 1.07 & 0.00 & 0.01 \\
\hline LOC219731 & 1569722_PM_s_at & uncharacterized LOC219731 & 1.07 & 0.00 & 0.01 \\
\hline PRR13 & 217794_PM_at & proline rich 13 & 1.07 & 0.00 & 0.01 \\
\hline--- & 233671_PM_at & --- & 1.07 & 0.00 & 0.01 \\
\hline CASP8 & 213373_PM_s_at & caspase 8 , apoptosis-related cysteine peptir & 1.07 & 0.00 & 0.01 \\
\hline ATP6V1E1 & 208678_PM_at & ATPase, $\mathrm{H}+$ transporting, lysosomal 31kDa, & 1.07 & 0.00 & 0.01 \\
\hline ATP6V0E1 & 200096_PM_s_at & ATPase, $\mathrm{H}+$ transporting, lysosomal 9kDa, V & 1.08 & 0.00 & 0.00 \\
\hline LOC440944 & 1557357_PM_at & uncharacterized LOC440944 & 1.08 & 0.00 & 0.00 \\
\hline--- & 234202_PM_at & --- & 1.08 & 0.00 & 0.00 \\
\hline CCDC67 & 1554072_PM_s_at & coiled-coil domain containing 67 & 1.08 & 0.00 & 0.00 \\
\hline SETD8 & 228443_PM_s_at & SET domain containing (lysine methyltransfi & 1.08 & 0.00 & 0.01 \\
\hline MRGPRX1 & 1553401_PM_at & MAS-related GPR, member X1 & 1.08 & 0.00 & 0.0 \\
\hline MBD4 & 209580_PM_s_at & methyl-CpG binding domain protein 4 & 1.08 & 0.00 & 0.01 \\
\hline BRK1 & 224023_PM_s_at & BRICK1, SCAR & 1.08 & 0.00 & \\
\hline
\end{tabular}




\begin{tabular}{|c|c|c|c|c|c|}
\hline PSG1 & 210196_PM_s_at & pregnancy specific beta-1-glycoprotein 1 & 1.08 & 0.00 & 0.01 \\
\hline--- & 1559313_PM_at & --- & 1.08 & 0.00 & 0.01 \\
\hline MOG & 214650_PM_x_at & myelin oligodendrocyte glycoprotein & 1.08 & 0.00 & 0.01 \\
\hline SETD8 & 225118_PM_at & SET domain containing (lysine methyltransf & 1.08 & 0.00 & 0.01 \\
\hline ZNF701 & 220242_PM_x_at & zinc finger protein 701 & 1.08 & 0.00 & 0.01 \\
\hline ZPLD1 & 1561969_PM_at & zona pellucida-like domain containing 1 & 1.08 & 0.00 & 0.01 \\
\hline TRIP4 & 203732_PM_at & thyroid hormone receptor interactor 4 & 1.08 & 0.00 & 0.01 \\
\hline GNG5 & 207157_PM_s_at & guanine nucleotide binding protein (G prot€ & 1.08 & 0.00 & 0.01 \\
\hline PDPN & 226658_PM_at & podoplanin & 1.08 & 0.00 & 0.01 \\
\hline LOC100652765 & 201171_PM_at & uncharacterized LOC100652765 & 1.09 & 0.00 & 0.00 \\
\hline MORF4L1 & 221381_PM_s_at & mortality factor 4 like 1 & 1.09 & 0.00 & 0.00 \\
\hline MBD4 & 214047_PM_s_at & methyl-CpG binding domain protein 4 & 1.09 & 0.00 & 0.00 \\
\hline ITM2B & 217732_PM_s_at & integral membrane protein $2 \mathrm{~B}$ & 1.09 & 0.00 & 0.00 \\
\hline WAC & 217742_PM_s_at & WW domain containing adaptor with coiled & 1.09 & 0.00 & 0.00 \\
\hline ARPC3 & 208736_PM_at & actin related protein 2 & 1.09 & 0.00 & 0.00 \\
\hline CLIC1 & 208659_PM_at & chloride intracellular channel 1 & 1.09 & 0.00 & 0.00 \\
\hline $\mathrm{H} 2 \mathrm{AFZ}$ & 213911_PM_s_at & H2A histone family, member $\mathrm{Z}$ & 1.09 & 0.00 & 0.00 \\
\hline BIN2 & 219191_PM_s_at & bridging integrator 2 & 1.09 & 0.00 & 0.00 \\
\hline TSG101 & 201758_PM_at & tumor susceptibility gene 101 & 1.09 & 0.00 & 0.00 \\
\hline FTH1P5 & 211628_PM_x_at & ferritin, heavy polypeptide 1 pseudogene 5 & 1.09 & 0.00 & 0.00 \\
\hline VAMP3 & 201336_PM_at & vesicle-associated membrane protein 3 (cel & 1.09 & 0.00 & 0.01 \\
\hline PHKB & 202739_PM_s_at & phosphorylase kinase, beta & 1.09 & 0.00 & 0.01 \\
\hline AP1G1 & 203350_PM_at & adaptor-related protein complex 1 , gamma & 1.09 & 0.00 & 0.01 \\
\hline--- & 1570645_PM_at & --- & 1.09 & 0.00 & 0.01 \\
\hline R3HCC1L & 228374_PM_at & R3H domain and coiled-coil containing 1-lik & 1.09 & 0.00 & 0.01 \\
\hline LINC00340 & 1562469_PM_at & long intergenic non-protein coding RNA $34 \mathrm{C}$ & 1.1 & 0.00 & 0.00 \\
\hline TMEM59 & 200620_PM_at & transmembrane protein 59 & 1.1 & 0.00 & 0.00 \\
\hline PPP2CA & 208652_PM_at & protein phosphatase 2 , catalytic subunit, all & 1.1 & 0.00 & 0.00 \\
\hline RAB11A & 200863_PM_s_at & RAB11A, member RAS oncogene family & 1.1 & 0.00 & 0.00 \\
\hline SLC12A6 & 226741_PM_at & solute carrier family 12 (potassium & 1.1 & 0.00 & 0.00 \\
\hline ITM2B & 217731_PM_s_at & integral membrane protein $2 \mathrm{~B}$ & 1.1 & 0.00 & 0.00 \\
\hline--- & 222202_PM_at & --- & 1.1 & 0.00 & 0.00 \\
\hline LCP2 & 205269_PM_at & Iymphocyte cytosolic protein 2 (SH2 domair & 1.1 & 0.00 & 0.00 \\
\hline
\end{tabular}




\begin{tabular}{|c|c|c|c|c|c|}
\hline SAT1 & 203455_PM_s_at & spermidine & 1.1 & 0.00 & 0.00 \\
\hline AMICA1 & 228094_PM_at & adhesion molecule, interacts with CXADR ar & 1.1 & 0.00 & 0.00 \\
\hline TINF2 & 220052_PM_s_at & TERF1 (TRF1)-interacting nuclear factor 2 & 1.1 & 0.00 & 0.00 \\
\hline SETX & 201964_PM_at & senataxin & 1.1 & 0.00 & 0.00 \\
\hline LOC100507699 & 202395_PM_at & vesicle-fusing ATPase-like & 1.1 & 0.00 & 0.00 \\
\hline LINC00616 & 1561098_PM_at at & long intergenic non-protein coding RNA $61 €$ & 1.1 & 0.00 & 0.00 \\
\hline OTP & 223835_PM_x_at & orthopedia homeobox & 1.1 & 0.00 & 0.00 \\
\hline CCNDBP1 & 223084_PM_s_at & cyclin D-type binding-protein 1 & 1.1 & 0.00 & 0.00 \\
\hline PLEK & 203471_PM_s_at & pleckstrin & 1.1 & 0.00 & 0.00 \\
\hline TUBA1A & 209118_PM_s_at & tubulin, alpha $1 a$ & 1.1 & 0.00 & 0.01 \\
\hline PHF20 & 209422_PM_at & PHD finger protein 20 & 1.1 & 0.00 & 0.01 \\
\hline CFHR3 & 1570228_PM_at & complement factor H-related 3 & 1.1 & 0.00 & 0.01 \\
\hline RANBP17 & 244198_PM_at & RAN binding protein 17 & 1.1 & 0.00 & 0.01 \\
\hline COG5 & 227089_PM_at & component of oligomeric golgi complex 5 & 1.1 & 0.00 & 0.01 \\
\hline CFTR & 234702_PM_x_at & cystic fibrosis transmembrane conductance & 1.1 & 0.00 & 0.01 \\
\hline FAM123C & 1553350_PM_at & family with sequence similarity $123 C$ & 1.1 & 0.00 & 0.01 \\
\hline NRD1 & 208709_PM_s_at & nardilysin ( $\mathrm{N}$-arginine dibasic convertase) & 1.1 & 0.00 & 0.01 \\
\hline PPCS & 218341_PM_at & phosphopantothenoylcysteine synthetase & 1.1 & 0.00 & 0.01 \\
\hline NCF2 & 209949_PM_at & neutrophil cytosolic factor 2 & 1.1 & 0.00 & 0.01 \\
\hline SUV42OH1 & 218242_PM_s_at & suppressor of variegation 4-20 homolog 1 (। & 1.1 & 0.00 & 0.01 \\
\hline UGT2B15 & 207392_PM_x_at & UDP glucuronosyltransferase 2 family, polyן & 1.1 & 0.00 & 0.01 \\
\hline LOC646214 & 236283_PM_x_at & p21 protein $(C d c 42$ & 1.1 & 0.00 & 0.01 \\
\hline GLUD2 & 210447_PM_at & glutamate dehydrogenase 2 & 1.1 & 0.00 & 0.01 \\
\hline RBM33 & 226732_PM_at & RNA binding motif protein 33 & 1.1 & 0.00 & 0.01 \\
\hline LOC100507463 & 1555852_PM_at & uncharacterized LOC100507463 & 1.1 & 0.00 & 0.01 \\
\hline SERPINB1 & 213572_PM_s_at & serpin peptidase inhibitor, clade B (ovalbun & 1.1 & 0.00 & 0.01 \\
\hline--- & 243412_PM_at & --- & 1.1 & 0.00 & 0.01 \\
\hline SETD2 & 215038_PM_s_at & SET domain containing 2 & 1.1 & 0.00 & 0.01 \\
\hline ADPRH & 207863_PM_at & ADP-ribosylarginine hydrolase & 1.1 & 0.00 & 0.01 \\
\hline MORF4L1 & 224561_PM_s_at & mortality factor 4 like 1 & 1.11 & 0.00 & 0.00 \\
\hline MAOB & 1561009_PM_at & Monoamine oxidase B & 1.11 & 0.00 & 0.00 \\
\hline PTTG1IP & 200677_PM_at & pituitary tumor-transforming 1 interacting $\mathrm{k}$ & 1.11 & 0.00 & 0.00 \\
\hline PLEKHB2 & 224121_PM_x_at & pleckstrin homology domain containing, far & 1.11 & 0.00 & 0.00 \\
\hline
\end{tabular}




\begin{tabular}{|c|c|c|c|c|c|}
\hline LRRFIP1 & 201862_PM_s_at & leucine rich repeat (in FLII) interacting prot€ & 1.11 & 0.00 & 0.00 \\
\hline $\mathrm{H} 2 \mathrm{AFZ}$ & 200853_PM_at & H2A histone family, member $\mathrm{Z}$ & 1.11 & 0.00 & 0.00 \\
\hline IDS & 212221_PM_x_at & iduronate 2 -sulfatase & 1.11 & 0.00 & 0.00 \\
\hline CPNE3 & 202119_PM_s_at & copine III & 1.11 & 0.00 & 0.00 \\
\hline--- & 1569812_PM_at & --- & 1.11 & 0.00 & 0.00 \\
\hline PRKG1 & 228396_PM_at & protein kinase, cGMP-dependent, type I & 1.11 & 0.00 & 0.00 \\
\hline TMUB2 & 218419_PM_s_at & transmembrane and ubiquitin-like domain c & 1.11 & 0.00 & 0.00 \\
\hline--- & 1569772_PM_x_at & --- & 1.11 & 0.00 & 0.00 \\
\hline SH3BGRL & 201312_PM_s_at & SH3 domain binding glutamic acid-rich prot & 1.11 & 0.00 & 0.00 \\
\hline--- & 1570181_PM_a_at & --- & 1.11 & 0.00 & 0.01 \\
\hline ATP6 & 242131_PM_at & ATP synthase F0 subunit 6 & 1.11 & 0.00 & 0.01 \\
\hline CLRN3 & 229777_PM_at & clarin 3 & 1.11 & 0.00 & 0.01 \\
\hline--- & 238016_PM_s_at & --- & 1.11 & 0.00 & 0.01 \\
\hline ZFP36 & 201531_PM_at & zinc finger protein $36, \mathrm{C} 3 \mathrm{H}$ type, homolog ( $r$ & 1.11 & 0.00 & 0.01 \\
\hline ELMO2 & 55692_PM_at & engulfment and cell motility 2 & 1.11 & 0.00 & 0.01 \\
\hline RFC2 & 1053_PM_at & replication factor $\mathrm{C}$ (activator 1 ) $2,40 \mathrm{kDa}$ & 1.11 & 0.00 & 0.01 \\
\hline GNAQ & 202615_PM_at & guanine nucleotide binding protein ( $G$ prot $\epsilon$ & 1.11 & 0.00 & 0.01 \\
\hline SERINC3 & 221471_PM_at & serine incorporator 3 & 1.11 & 0.00 & 0.01 \\
\hline DHRS7B & 220690_PM_s_at & dehydrogenase & 1.11 & 0.00 & 0.01 \\
\hline PPM1M & 226074_PM_at & protein phosphatase, $\mathrm{Mg} 2+$ & 1.11 & 0.00 & 0.01 \\
\hline GOLGA7 & 1554167_PM_a_at & golgin A7 & 1.11 & 0.00 & 0.01 \\
\hline--- & 237359_PM_at & --- & 1.11 & 0.00 & 0.01 \\
\hline BZW1 & 200776_PM_s_at & basic leucine zipper and W2 domains 1 & 1.11 & 0.00 & 0.01 \\
\hline NCOA1 & 209106_PM_at & nuclear receptor coactivator 1 & 1.11 & 0.00 & 0.01 \\
\hline YWHAH & 201020_PM_at & tyrosine 3-monooxygenase & 1.11 & 0.00 & 0.01 \\
\hline MIR21 & 220990_PM_s_at & microRNA 21 & 1.11 & 0.00 & 0.01 \\
\hline LINC00324 & 230012_PM_at & long intergenic non-protein coding RNA $32 \angle$ & 1.11 & 0.00 & 0.01 \\
\hline SRGN & 201859_PM_at & serglycin & 1.12 & 0.00 & 0.00 \\
\hline TINF2 & 223776_PM_x_at & TERF1 (TRF1)-interacting nuclear factor 2 & 1.12 & 0.00 & 0.00 \\
\hline SERPINB1 & 228726_PM_at & serpin peptidase inhibitor, clade B (ovalbun & 1.12 & 0.00 & 0.00 \\
\hline RPS6KA3 & 203843_PM_at & ribosomal protein S6 kinase, 90kDa, polype & 1.12 & 0.00 & 0.00 \\
\hline AP1M2 & 65517_PM_at & adaptor-related protein complex 1, mu 2 su & 1.12 & 0.00 & 0.00 \\
\hline GIT2 & 225558_PM_at & G protein-coupled receptor kinase interacti & 1.12 & 0.00 & 0.00 \\
\hline
\end{tabular}




\begin{tabular}{|c|c|c|c|c|}
\hline РТВР3 & 224617_PM_at & polypyrimidine tract binding protein 3 & 1.12 & 0.00 \\
\hline KPNA2 & 201088_PM_at & karyopherin alpha 2 (RAG cohort 1, importi। & 1.12 & 0.00 \\
\hline RAB27A & 222294_PM_s_at & RAB27A, member RAS oncogene family & 1.12 & 0.00 \\
\hline PSEN1 & 226577_PM_at & presenilin 1 & 1.12 & 0.00 \\
\hline POLR2C & 214263_PM_x_at & polymerase (RNA) II (DNA directed) polyper & 1.12 & 0.00 \\
\hline DIAPH3 & 232596_PM_at & diaphanous homolog 3 (Drosophila) & 1.12 & 0.00 \\
\hline PLXNA2 & 227032_PM_at & plexin A2 & 1.12 & 0.00 \\
\hline CDCA3 & 223307_PM_at & cell division cycle associated 3 & 1.12 & 0.00 \\
\hline REEP5 & 208872_PM_s_at & receptor accessory protein 5 & 1.12 & 0.00 \\
\hline--- & 234371_PM_at & --- & 1.12 & 0.00 \\
\hline RNF135 & 223591_PM_at & ring finger protein 135 & 1.12 & 0.00 \\
\hline CDKN2D & 210240_PM_s_at & cyclin-dependent kinase inhibitor 2D (p19, i & 1.12 & 0.00 \\
\hline LOC100505487 & 225197_PM_at & uncharacterized LOC100505487 & 1.12 & 0.00 \\
\hline GSTO1 & 201470_PM_at & glutathione S-transferase omega 1 & 1.12 & 0.00 \\
\hline MSRB3 & 238583_PM_at & methionine sulfoxide reductase B3 & 1.12 & 0.00 \\
\hline FAM5B & 214822_PM_at & family with sequence similarity 5 , member I & 1.12 & 0.00 \\
\hline LOC100287223 & 227396_PM_at & uncharacterized LOC100287223 & 1.12 & 0.00 \\
\hline C20orf43 & 217737_PM_x_at & chromosome 20 open reading frame 43 & 1.12 & 0.00 \\
\hline SFN & 33323_PM_r_at & stratifin & 1.12 & 0.00 \\
\hline NF1 & 204323_PM_x_at & neurofibromin 1 & 1.12 & 0.00 \\
\hline ITGAM & 205786_PM_s_at & integrin, alpha M (complement component & 1.12 & 0.00 \\
\hline CTAGE5 & 215930_PM_s_at & CTAGE family, member 5 & 1.12 & 0.00 \\
\hline CKLF & 223451_PM_s_at & chemokine-like factor & 1.12 & 0.00 \\
\hline SCN1A & 210383_PM_at & sodium channel, voltage-gated, type I, alphi & 1.12 & 0.00 \\
\hline SDHD & 202026_PM_at & succinate dehydrogenase complex, subunit & 1.12 & 0.00 \\
\hline GADD45B & 209304_PM_x_at & growth arrest and DNA-damage-inducible, & 1.12 & 0.00 \\
\hline ERG & 211626_PM_x_at & v-ets erythroblastosis virus E26 oncogene $h$ & 1.12 & 0.00 \\
\hline--- & 242950_PM_x_at & --- & 1.12 & 0.00 \\
\hline CACNA1D & 210108_PM_at & calcium channel, voltage-dependent, L type & 1.12 & 0.00 \\
\hline MAN2A2 & 202032_PM_s_at & mannosidase, alpha, class $2 \mathrm{~A}$, member 2 & 1.12 & 0.00 \\
\hline USP8 & 229501_PM_s_at & ubiquitin specific peptidase 8 & 1.13 & 0.00 \\
\hline MYL12A & 201319_PM_at & myosin, light chain $12 \mathrm{~A}$, regulatory, non-sar & 1.13 & 0.00 \\
\hline OSBPL8 & 212582_PM_at & oxysterol binding protein-like 8 & 1.13 & 0.00 \\
\hline
\end{tabular}




\begin{tabular}{|c|c|c|c|c|c|}
\hline ZNF552 & 219741_PM_x_at & zinc finger protein 552 & 1.13 & 0.00 & 0.00 \\
\hline FGR & 208438_PM_s_at & Gardner-Rasheed feline sarcoma viral (v-fgr & 1.13 & 0.00 & 0.00 \\
\hline MYL6 & 212082_PM_s_at & myosin, light chain 6 , alkali, smooth muscle & 1.13 & 0.00 & 0.00 \\
\hline SCPEP1 & 218217_PM_at & serine carboxypeptidase 1 & 1.13 & 0.00 & 0.00 \\
\hline SH3GLB1 & 209090_PM_s_at & SH3-domain GRB2-like endophilin B1 & 1.13 & 0.00 & 0.00 \\
\hline CTBP2 & 210554_PM_s_at & C-terminal binding protein 2 & 1.13 & 0.00 & 0.00 \\
\hline MKLN1 & 225526_PM_at & muskelin 1, intracellular mediator containin & 1.13 & 0.00 & 0.00 \\
\hline--- & 226391_PM_at & --- & 1.13 & 0.00 & 0.00 \\
\hline CYTH4 & 211521_PM_s_at & cytohesin 4 & 1.13 & 0.00 & 0.00 \\
\hline LRMP & 204674_PM_at & lymphoid-restricted membrane protein & 1.13 & 0.00 & 0.00 \\
\hline PGM1 & 201968_PM_s_at & phosphoglucomutase 1 & 1.13 & 0.00 & 0.00 \\
\hline HEXIM1 & 202815_PM_s_at & hexamethylene bis-acetamide inducible 1 & 1.13 & 0.00 & 0.00 \\
\hline KBTBD11 & 204301_PM_at & kelch repeat and BTB (POZ) domain contain & 1.13 & 0.00 & 0.00 \\
\hline NOL3 & 59625_PM_at & nucleolar protein 3 (apoptosis repressor wit & 1.13 & 0.00 & 0.00 \\
\hline TOR4A & 233589_PM_x_at & torsin family 4 , member $A$ & 1.13 & 0.00 & 0.00 \\
\hline FERMT3 & 223303_PM_at & fermitin family member 3 & 1.13 & 0.00 & 0.00 \\
\hline PELO & 218472_PM_s_at & pelota homolog (Drosophila) & 1.13 & 0.00 & 0.00 \\
\hline MAP3K5 & 203837_PM_at & mitogen-activated protein kinase kinase kin & 1.13 & 0.00 & 0.00 \\
\hline SLC39A14 & 1555433_PM_at & solute carrier family 39 (zinc transporter), $n$ & 1.13 & 0.00 & 0.01 \\
\hline IL17RA & 229295_PM_at & interleukin 17 receptor $A$ & 1.13 & 0.00 & 0.01 \\
\hline RAB27A & 235766_PM_x_at & RAB27A, member RAS oncogene family & 1.13 & 0.00 & 0.01 \\
\hline CHMP3 & 222436_PM_s_at & charged multivesicular body protein 3 & 1.13 & 0.00 & 0.01 \\
\hline MAPK1 & 1552263_PM_at & mitogen-activated protein kinase 1 & 1.13 & 0.00 & 0.01 \\
\hline AlF1 & 213095_PM_x_at & allograft inflammatory factor 1 & 1.13 & 0.00 & 0.01 \\
\hline--- & 1560034_PM_a_at & --- & 1.13 & 0.00 & 0.01 \\
\hline DNAJC5 & 224613_PM_s_at & DnaJ (Hsp40) homolog, subfamily C, membe & 1.13 & 0.00 & 0.01 \\
\hline BRCC3 & 221196_PM_x_at & BRCA1 & 1.13 & 0.00 & 0.01 \\
\hline PRELID1 & 223032_PM_x_at & PRELI domain containing 1 & 1.13 & 0.00 & 0.01 \\
\hline ITPR2 & 202661_PM_at & inositol 1,4,5-trisphosphate receptor, type: & 1.13 & 0.00 & 0.01 \\
\hline SLCO3A1 & 219229_PM_at & solute carrier organic anion transporter far & 1.13 & 0.00 & 0.01 \\
\hline MYL12B & 221474_PM_at & myosin, light chain $12 \mathrm{~B}$, regulatory & 1.14 & 0.00 & 0.00 \\
\hline EVI2B & 211742_PM_s_at & ecotropic viral integration site $2 \mathrm{~B}$ & 1.14 & 0.00 & 0.00 \\
\hline PICALM & 212506_PM_at & phosphatidylinositol binding clathrin assem & 1.14 & 0.00 & 0.00 \\
\hline
\end{tabular}




\begin{tabular}{|c|c|c|c|c|c|}
\hline TINF2 & 224809_PM_x_at & TERF1 (TRF1)-interacting nuclear factor 2 & 1.14 & 0.00 & 0.00 \\
\hline CAMK2G & 212757_PM_s_at & calcium & 1.14 & 0.00 & 0.00 \\
\hline CDC42EP3 & 225685_PM_at & CDC42 effector protein (Rho GTPase bindin! & 1.14 & 0.00 & 0.00 \\
\hline MCMBP & 222464_PM_s_at & minichromosome maintenance complex bir & 1.14 & 0.00 & 0.00 \\
\hline TNFAIP8L2 & 223583_PM_at & tumor necrosis factor, alpha-induced protei & 1.14 & 0.00 & 0.00 \\
\hline BID & 227143_PM_s_at & $\mathrm{BH} 3$ interacting domain death agonist & 1.14 & 0.00 & 0.00 \\
\hline STAG1 & 202294_PM_at & stromal antigen 1 & 1.14 & 0.00 & 0.00 \\
\hline LRRFIP1 & 227391_PM_x_at & leucine rich repeat (in FLII) interacting prot $\epsilon$ & 1.14 & 0.00 & 0.00 \\
\hline LRMP & 35974_PM_at & lymphoid-restricted membrane protein & 1.14 & 0.00 & 0.00 \\
\hline GJA9-MYCBP & 203359_PM_s_at & GJA9-MYCBP readthrough & 1.14 & 0.00 & 0.00 \\
\hline INPP5A & 203006_PM_at & inositol polyphosphate-5-phosphatase, 40k & 1.14 & 0.00 & 0.00 \\
\hline RGS19 & 204336_PM_s_at & regulator of G-protein signaling 19 & 1.14 & 0.00 & 0.00 \\
\hline--- & 229934_PM_at & --- & 1.14 & 0.00 & 0.00 \\
\hline CNIH4 & 228306_PM_at & cornichon homolog 4 (Drosophila) & 1.14 & 0.00 & 0.00 \\
\hline PIK3R5 & 227553_PM_at & Phosphoinositide-3-kinase, regulatory subu & 1.14 & 0.00 & 0.00 \\
\hline LAMP2 & 200821_PM_at & Iysosomal-associated membrane protein 2 & 1.14 & 0.00 & 0.00 \\
\hline FNIP1 & 228768_PM_at & folliculin interacting protein 1 & 1.14 & 0.00 & 0.00 \\
\hline--- & 232607_PM_at & --- & 1.14 & 0.00 & 0.00 \\
\hline ANXA11 & 228727_PM_at & annexin A11 & 1.14 & 0.00 & 0.00 \\
\hline PIK3R5 & 227645_PM_at & phosphoinositide-3-kinase, regulatory subu & 1.14 & 0.00 & 0.00 \\
\hline ERG & 213541_PM_s_at & v-ets erythroblastosis virus E26 oncogene $h$ & 1.14 & 0.00 & 0.00 \\
\hline SERPINB1 & 212268_PM_at & serpin peptidase inhibitor, clade B (ovalbun & 1.14 & 0.00 & 0.00 \\
\hline FAM129A & 217967_PM_s_at & family with sequence similarity 129 , memb & 1.14 & 0.00 & 0.00 \\
\hline CFLAR & 235427_PM_at & CASP8 and FADD-like apoptosis regulator & 1.14 & 0.00 & 0.00 \\
\hline $\mathrm{SSH} 3$ & 51192_PM_at & slingshot homolog 3 (Drosophila) & 1.14 & 0.00 & 0.00 \\
\hline ACAA1 & 214274_PM_s_at & acetyl-CoA acyltransferase 1 & 1.14 & 0.00 & 0.00 \\
\hline BRI3 & 223376_PM_s_at & brain protein 13 & 1.14 & 0.00 & 0.00 \\
\hline ZNF33A & 231864_PM_at & zinc finger protein $33 \mathrm{~A}$ & 1.14 & 0.00 & 0.00 \\
\hline STRN & 236980_PM_at & striatin, calmodulin binding protein & 1.14 & 0.00 & 0.01 \\
\hline C14orf101 & 225675_PM_at & chromosome 14 open reading frame 101 & 1.14 & 0.00 & 0.01 \\
\hline--- & 235843_PM_at & --- & 1.14 & 0.00 & 0.01 \\
\hline CEBPA & 204039_PM_at & CCAAT & 1.14 & 0.00 & 0.01 \\
\hline CHMP1B & 218178_PM_s_at & charged multivesicular body protein $1 \mathrm{~B}$ & 1.14 & 0.00 & 0.01 \\
\hline
\end{tabular}




\begin{tabular}{|c|c|c|c|c|c|}
\hline MAPK14 & 202530_PM_at & mitogen-activated protein kinase 14 & 1.14 & 0.00 & 0.01 \\
\hline CKLF & 221058_PM_s_at & chemokine-like factor & 1.14 & 0.00 & 0.01 \\
\hline COA1 & 239490_PM_at & cytochrome $\mathrm{C}$ oxidase assembly factor 1 ho & 1.14 & 0.00 & 0.01 \\
\hline PBLD & 219543_PM_at & phenazine biosynthesis-like protein domain & 1.14 & 0.00 & 0.01 \\
\hline TLR1 & 210176_PM_at & toll-like receptor 1 & 1.14 & 0.00 & 0.01 \\
\hline $\mathrm{DCP} 2$ & 244777_PM_at & DCP2 decapping enzyme homolog (S. cerevi & 1.14 & 0.00 & 0.01 \\
\hline DEF8 & 219646_PM_at & differentially expressed in FDCP 8 homolog & 1.14 & 0.00 & 0.01 \\
\hline FLOT2 & 201350_PM_at & flotillin 2 & 1.14 & 0.00 & 0.01 \\
\hline ACPP & 237030_PM_at & acid phosphatase, prostate & 1.14 & 0.00 & 0.01 \\
\hline TPMT & 203671_PM_at & thiopurine S-methyltransferase & 1.14 & 0.00 & 0.01 \\
\hline ASAP1 & 224791_PM_at & ArfGAP with SH3 domain, ankyrin repeat an & 1.15 & 0.00 & 0.00 \\
\hline ATXN1 & 203232_PM_s_at & ataxin 1 & 1.15 & 0.00 & 0.00 \\
\hline CTSS & 202901_PM_x_at & cathepsin S & 1.15 & 0.00 & 0.00 \\
\hline CENPBD1 & 223728_PM_at & CENPB DNA-binding domains containing 1 & 1.15 & 0.00 & 0.00 \\
\hline RGS14 & 38290_PM_at & regulator of G-protein signaling 14 & 1.15 & 0.00 & 0.00 \\
\hline HBXIP & 202300_PM_at & hepatitis $B$ virus $x$ interacting protein & 1.15 & 0.00 & 0.00 \\
\hline FAM65B & 209829_PM_at & family with sequence similarity 65, member & 1.15 & 0.00 & 0.00 \\
\hline PROK2 & 232629_PM_at & prokineticin 2 & 1.15 & 0.00 & 0.00 \\
\hline GYG1 & 211275_PM_s_at & glycogenin 1 & 1.15 & 0.00 & 0.00 \\
\hline LOC100129034 & 225214_PM_at & uncharacterized LOC100129034 & 1.15 & 0.00 & 0.00 \\
\hline GDE1 & 226214_PM_at & glycerophosphodiester phosphodiesterase & 1.15 & 0.00 & 0.00 \\
\hline CSF2RA & 211286_PM_x_at & colony stimulating factor 2 receptor, alpha, & 1.15 & 0.00 & 0.00 \\
\hline NFE2L2 & 201146_PM_at & nuclear factor (erythroid-derived 2)-like 2 & 1.15 & 0.00 & 0.00 \\
\hline CACNG8 & 231355_PM_at & calcium channel, voltage-dependent, gamm & 1.15 & 0.00 & 0.00 \\
\hline STOM & 201062_PM_at & stomatin & 1.15 & 0.00 & 0.00 \\
\hline ARL8B & 222442_PM_s_at & ADP-ribosylation factor-like $8 \mathrm{~B}$ & 1.15 & 0.00 & 0.00 \\
\hline ZNF586 & 219711_PM_at & zinc finger protein 586 & 1.15 & 0.00 & 0.00 \\
\hline FAM32A & 201863_PM_at & family with sequence similarity 32 , member & 1.15 & 0.00 & 0.00 \\
\hline--- & 216012_PM_at & --- & 1.15 & 0.00 & 0.00 \\
\hline GALNT7 & 222587_PM_s_at & UDP-N-acetyl-alpha-D-galactosamine:polyp & 1.15 & 0.00 & 0.00 \\
\hline UBXN2B & 212934_PM_at & UBX domain protein $2 \mathrm{~B}$ & 1.15 & 0.00 & 0.00 \\
\hline--- & 240064_PM_at & --- & 1.15 & 0.00 & 0.00 \\
\hline PGM2 & 223738_PM_s_at & phosphoglucomutase 2 & 1.15 & 0.00 & 0.01 \\
\hline
\end{tabular}




\begin{tabular}{|c|c|c|c|c|c|}
\hline GBA & 210589_PM_s_at & glucosidase, beta, acid & 1.15 & 0.00 & 0.01 \\
\hline C10orf128 & 1561890_PM_at & chromosome 10 open reading frame 128 & 1.15 & 0.00 & 0.01 \\
\hline--- & 1557099_PM_at & --- & 1.15 & 0.00 & 0.01 \\
\hline EEF1A1 & 1557120_PM_at & Eukaryotic translation elongation factor $1 \mathrm{a}$ & 1.15 & 0.00 & 0.01 \\
\hline SMARCD3 & 204099_PM_at & SWI & 1.15 & 0.00 & 0.01 \\
\hline CCNYL1 & 227280_PM_s_at & cyclin Y-like 1 & 1.15 & 0.00 & 0.01 \\
\hline CMTM4 & 224998_PM_at & CKLF-like MARVEL transmembrane domain & 1.15 & 0.00 & 0.01 \\
\hline PACSIN2 & 201651_PM_s_at & protein kinase $C$ and casein kinase substrat & 1.15 & 0.00 & 0.01 \\
\hline UBE2J1 & 217824_PM_at & ubiquitin-conjugating enzyme E2, J1 & 1.15 & 0.00 & 0.01 \\
\hline STOM & 201061_PM_s_at & stomatin & 1.15 & 0.00 & 0.01 \\
\hline STX3 & 209238_PM_at & syntaxin 3 & 1.15 & 0.00 & 0.01 \\
\hline ATP5J2 & 202961_PM_s_at & ATP synthase, $\mathrm{H}+$ transporting, mitochondri & 1.15 & 0.00 & 0.01 \\
\hline MGST1 & 224918_PM_x_at & microsomal glutathione S-transferase 1 & 1.15 & 0.00 & 0.01 \\
\hline CYBB & 233538_PM_s_at & Cytochrome b-245, beta polypeptide & 1.15 & 0.00 & 0.01 \\
\hline SOS2 & 217644_PM_s_at & son of sevenless homolog 2 (Drosophila) & 1.15 & 0.00 & 0.01 \\
\hline XPR1 & 226615_PM_at & xenotropic and polytropic retrovirus recept & 1.15 & 0.00 & 0.01 \\
\hline $\mathrm{SH} 2 \mathrm{D} 3 \mathrm{C}$ & 226673_PM_at & $\mathrm{SH} 2$ domain containing $3 \mathrm{C}$ & 1.15 & 0.00 & 0.01 \\
\hline $\mathrm{MCU}$ & 225320_PM_at & mitochondrial calcium uniporter & 1.16 & 0.00 & 0.00 \\
\hline DAPP1 & 219290_PM_x_at & dual adaptor of phosphotyrosine and 3-pho & 1.16 & 0.00 & 0.00 \\
\hline LAT2 & 221581_PM_s_at & linker for activation of T cells family, memb & 1.16 & 0.00 & 0.00 \\
\hline--- & 1564121_PM_at & --- & 1.16 & 0.00 & 0.00 \\
\hline GJA9-MYCBP & 203360_PM_s_at & GJA9-MYCBP readthrough & 1.16 & 0.00 & 0.00 \\
\hline SLC2A3 & 202499_PM_s_at & solute carrier family 2 (facilitated glucose $\operatorname{tr}$ & 1.16 & 0.00 & 0.00 \\
\hline LINC00525 & 1553708_PM_at & long intergenic non-protein coding RNA 525 & 1.16 & 0.00 & 0.00 \\
\hline $\mathrm{SSH} 3$ & 219241_PM_x_at & slingshot homolog 3 (Drosophila) & 1.16 & 0.00 & 0.00 \\
\hline DNTTIP1 & 224825_PM_at & deoxynucleotidyltransferase, terminal, inteı & 1.16 & 0.00 & 0.00 \\
\hline ACVR1B & 213198_PM_at & activin $A$ receptor, type IB & 1.16 & 0.00 & 0.00 \\
\hline ZEB2 & 235593_PM_at & zinc finger E-box binding homeobox 2 & 1.16 & 0.00 & 0.00 \\
\hline PLBD1 & 218454_PM_at & phospholipase B domain containing 1 & 1.16 & 0.00 & 0.01 \\
\hline PPP1R3D & 204554_PM_at & protein phosphatase 1 , regulatory subunit $\equiv$ & 1.16 & 0.00 & 0.01 \\
\hline MTX1 & 210386_PM_s_at & metaxin 1 & 1.16 & 0.00 & 0.01 \\
\hline LAMP2 & 203042_PM_at & Iysosomal-associated membrane protein 2 & 1.16 & 0.00 & 0.01 \\
\hline WIPI1 & 213836_PM_s_at & WD repeat domain, phosphoinositide interć & 1.16 & 0.00 & 0.01 \\
\hline
\end{tabular}




\begin{tabular}{|c|c|c|c|c|c|}
\hline ELF2 & 203822_PM_S_at & E74-like factor 2 (ets domain transcription $f$ & 1.16 & 0.00 & 0.01 \\
\hline FOXO3 & 224891_PM_at & forkhead box $\mathrm{O3}$ & 1.16 & 0.00 & 0.01 \\
\hline--- & 1554723_PM_X_at & --- & 1.16 & 0.00 & 0.01 \\
\hline NDUFA1 & 202298_PM_at & NADH dehydrogenase (ubiquinone) 1 alpha & 1.16 & 0.00 & 0.01 \\
\hline-- & 237451_PM_x_at & -- & 1.16 & 0.00 & 0.01 \\
\hline SF3B14 & 223416_PM_at & splicing factor $3 \mathrm{~B}, 14 \mathrm{kDa}$ subunit & 1.16 & 0.00 & 0.01 \\
\hline TMEM189 & 201003_PM_x_at & transmembrane protein 189 & 1.16 & 0.00 & 0.01 \\
\hline NEDD9 & 202149_PM_at & neural precursor cell expressed, developme & 1.16 & 0.00 & 0.01 \\
\hline LILRA2 & 207857_PM_at & leukocyte immunoglobulin-like receptor, su & 1.16 & 0.00 & 0.01 \\
\hline SUCNR1 & 223939_PM_at & succinate receptor 1 & 1.16 & 0.00 & 0.01 \\
\hline PCOLCE2 & 219295_PM_S_at & procollagen C-endopeptidase enhancer 2 & 1.16 & 0.00 & 0.01 \\
\hline TXN & 216609_PM_at & thioredoxin & 1.16 & 0.00 & 0.02 \\
\hline APBB1IP & 230925_PM_at & amyloid beta (A4) precursor protein-bindin & 1.16 & 0.00 & 0.02 \\
\hline LRRC25 & 1559502_PM_s_at & leucine rich repeat containing 25 & 1.16 & 0.00 & 0.02 \\
\hline ENTPD1 & 207691_PM_x_at & ectonucleoside triphosphate diphosphohyd & 1.16 & 0.00 & 0.02 \\
\hline CHMP3 & 217837_PM_s_at & charged multivesicular body protein 3 & 1.17 & 0.00 & 0.00 \\
\hline HBXIP & 202299_PM_S_at & hepatitis $B$ virus $x$ interacting protein & 1.17 & 0.00 & 0.00 \\
\hline RAB32 & 204214_PM_S_at & RAB32, member RAS oncogene family & 1.17 & 0.00 & 0.00 \\
\hline LEPROT & 202377_PM_at & leptin receptor overlapping transcript & 1.17 & 0.00 & 0.00 \\
\hline PPP2R5A & 202187_PM_S_at & protein phosphatase 2 , regulatory subunit $\mathrm{E}$ & 1.17 & 0.00 & 0.00 \\
\hline GLIPR1 & 226136_PM_at & GLI pathogenesis-related 1 & 1.17 & 0.00 & 0.00 \\
\hline--- & 217352_PM_at & --- & 1.17 & 0.00 & 0.00 \\
\hline STEAP4 & 225987_PM_at & STEAP family member 4 & 1.17 & 0.00 & 0.00 \\
\hline--- & 229602_PM_at & --- & 1.17 & 0.00 & 0.00 \\
\hline AZIN1 & 201772_PM_at & antizyme inhibitor 1 & 1.17 & 0.00 & 0.00 \\
\hline LOC100509484 & 209122_PM_at & uncharacterized LOC100509484 & 1.17 & 0.00 & 0.00 \\
\hline AGFG1 & 218092_PM_S_at & ArfGAP with FG repeats 1 & 1.17 & 0.00 & 0.00 \\
\hline $\mathrm{SSH} 3$ & 219919_PM_s_at & slingshot homolog 3 (Drosophila) & 1.17 & 0.00 & 0.00 \\
\hline C1RL & 218983_PM_at & complement component $1, r$ subcomponen & 1.17 & 0.00 & 0.00 \\
\hline PCTP & 218676_PM_S_at & phosphatidylcholine transfer protein & 1.17 & 0.00 & 0.00 \\
\hline BRCC3 & 229436_PM_X_at & BRCA1 & 1.17 & 0.00 & 0.00 \\
\hline SNTB2 & 226685_PM_at & syntrophin, beta 2 (dystrophin-associa & 1.17 & 0.00 & 0.00 \\
\hline TNFSF14 & 233935_PM_at & Tumor necrosis factor (ligand) superfamily, & 1.17 & 0.00 & 0.00 \\
\hline
\end{tabular}




\begin{tabular}{|c|c|c|c|c|c|}
\hline GLIPR2 & 225604_PM_s_at & GLI pathogenesis-related 2 & 1.17 & 0.00 & 0.00 \\
\hline UBL5 & 218011_PM_at & ubiquitin-like 5 & 1.17 & 0.00 & 0.00 \\
\hline XPO6 & 214784_PM_x_at & exportin 6 & 1.17 & 0.00 & 0.00 \\
\hline ASAP1 & 221039_PM_s_at & ArfGAP with $\mathrm{SH} 3$ domain, ankyrin repeat an & 1.17 & 0.00 & 0.00 \\
\hline PPT1 & 200975_PM_at & palmitoyl-protein thioesterase 1 & 1.17 & 0.00 & 0.00 \\
\hline SRPK1 & 202200_PM_s_at & SRSF protein kinase 1 & 1.17 & 0.00 & 0.00 \\
\hline KIAA2018 & 227433_PM_at & KIAA2018 & 1.17 & 0.00 & 0.00 \\
\hline $\mathrm{CPQ}$ & 203501_PM_at & carboxypeptidase Q & 1.17 & 0.00 & 0.00 \\
\hline MKNK1 & 209467_PM_s_at & MAP kinase interacting serine & 1.17 & 0.00 & 0.00 \\
\hline CUL4B & 202213_PM_s_at & cullin 4B & 1.17 & 0.00 & 0.00 \\
\hline PPP3CA & 202429_PM_s_at & protein phosphatase 3 , catalytic subunit, al| & 1.17 & 0.00 & 0.00 \\
\hline PLD1 & 226636_PM_at & phospholipase D1, phosphatidylcholine-spe & 1.17 & 0.00 & 0.00 \\
\hline PLIN4 & 228409_PM_at & perilipin 4 & 1.17 & 0.00 & 0.00 \\
\hline VTI1B & 225926_PM_at & vesicle transport through interaction with $\mathrm{t}$. & 1.17 & 0.00 & 0.00 \\
\hline MGST1 & 231736_PM_x_at & microsomal glutathione S-transferase 1 & 1.17 & 0.00 & 0.01 \\
\hline CYB5R4 & 219079_PM_at & cytochrome b5 reductase 4 & 1.17 & 0.00 & 0.01 \\
\hline LAMP2 & 203041_PM_s_at & lysosomal-associated membrane protein 2 & 1.17 & 0.00 & 0.01 \\
\hline SKAP2 & 225639_PM_at & src kinase associated phosphoprotein 2 & 1.17 & 0.00 & 0.01 \\
\hline FRAT1 & 219889_PM_at & frequently rearranged in advanced T-cell lyr & 1.17 & 0.00 & 0.01 \\
\hline VEPH1 & 229759_PM_s_at & ventricular zone expressed $\mathrm{PH}$ domain hom & 1.17 & 0.00 & 0.01 \\
\hline DCBLD2 & 224911_PM_s_at & discoidin, CUB and LCCL domain containing & 1.17 & 0.00 & 0.01 \\
\hline HK2 & 202934_PM_at & hexokinase 2 & 1.17 & 0.00 & 0.01 \\
\hline--- & 243115_PM_at & --- & 1.17 & 0.00 & 0.01 \\
\hline IFNAR1 & 204191_PM_at & interferon (alpha, beta and omega) recepto & 1.17 & 0.00 & 0.01 \\
\hline FRY & 214319_PM_at & furry homolog (Drosophila) & 1.17 & 0.00 & 0.01 \\
\hline CSF2RA & 207085_PM_x_at & colony stimulating factor 2 receptor, alpha, & 1.17 & 0.00 & 0.01 \\
\hline PDK3 & 221957_PM_at & pyruvate dehydrogenase kinase, isozyme 3 & 1.17 & 0.00 & 0.01 \\
\hline C11orf82 & 228281_PM_at & chromosome 11 open reading frame 82 & 1.17 & 0.00 & 0.01 \\
\hline OSCAR & 1554503_PM_a_at & osteoclast associated, immunoglobulin-like & 1.17 & 0.00 & 0.01 \\
\hline TBC1D1 & 1568713_PM_a_at & TBC1 (tre-2 & 1.17 & 0.00 & 0.01 \\
\hline ENTPD1 & 209474_PM_s_at & ectonucleoside triphosphate diphosphohyd & 1.17 & 0.00 & 0.01 \\
\hline B3GNT2 & 219326_PM_s_at & UDP-GIcNAc:betaGal beta-1,3-N-acetylglucc & 1.17 & 0.00 & 0.01 \\
\hline MYD88 & 209124_PM_at & myeloid differentiation primary response g $\epsilon$ & 1.18 & 0.00 & 0.00 \\
\hline
\end{tabular}




\begin{tabular}{|c|c|c|c|c|c|}
\hline GNAQ & 224861_PM_at & guanine nucleotide binding protein (G prot $\epsilon$ & 1.18 & 0.00 & 0.00 \\
\hline LOC100507507 & 241353_PM_s_at & uncharacterized LOC100507507 & 1.18 & 0.00 & 0.00 \\
\hline LRRC57 & 229232_PM_at & leucine rich repeat containing 57 & 1.18 & 0.00 & 0.00 \\
\hline IFNAR1 & 225661_PM_at & interferon (alpha, beta and omega) recepto & 1.18 & 0.00 & 0.00 \\
\hline AQP9 & 205568_PM_at & aquaporin 9 & 1.18 & 0.00 & 0.00 \\
\hline FAM47E & 203986_PM_at & family with sequence similarity 47 , member & 1.18 & 0.00 & 0.00 \\
\hline FADD & 202535_PM_at & Fas (TNFRSF6)-associated via death domain & 1.18 & 0.00 & 0.00 \\
\hline KCNE3 & 227647_PM_at & potassium voltage-gated channel, Isk-relate & 1.18 & 0.00 & 0.00 \\
\hline TANK & 207616_PM_s_at & TRAF family member-associated NFKB activ & 1.18 & 0.00 & 0.00 \\
\hline SUSD1 & 226264_PM_at & sushi domain containing 1 & 1.18 & 0.00 & 0.00 \\
\hline BID & 204493_PM_at & $\mathrm{BH} 3$ interacting domain death agonist & 1.18 & 0.00 & 0.00 \\
\hline DENND3 & 212975_PM_at & DENN & 1.18 & 0.00 & 0.00 \\
\hline AGTPBP1 & 204500_PM_s_at & ATP & 1.18 & 0.00 & 0.00 \\
\hline CCDC30 & 230897_PM_at & coiled-coil domain containing 30 & 1.18 & 0.00 & 0.00 \\
\hline CHD9 & 212616_PM_at & chromodomain helicase DNA binding protei & 1.18 & 0.00 & 0.00 \\
\hline SNTB1 & 226438_PM_at & syntrophin, beta 1 (dystrophin-associated p & 1.18 & 0.00 & 0.00 \\
\hline CDC42EP3 & 209287_PM_s_at & CDC42 effector protein (Rho GTPase bindin! & 1.18 & 0.00 & 0.00 \\
\hline FGD4 & 230559_PM_x_at & FYVE, RhoGEF and PH domain containing 4 & 1.18 & 0.00 & 0.01 \\
\hline ANKRD12 & 216550_PM_x_at & ankyrin repeat domain 12 & 1.18 & 0.00 & 0.01 \\
\hline STRN & 236388_PM_at & striatin, calmodulin binding protein & 1.18 & 0.00 & 0.01 \\
\hline TRIQK & 225603_PM_s_at & triple QxxK & 1.18 & 0.00 & 0.01 \\
\hline TXN & 208864_PM_s_at & thioredoxin & 1.18 & 0.00 & 0.01 \\
\hline RRP12 & 216913_PM_s_at & ribosomal RNA processing 12 homolog (S. c & 1.18 & 0.00 & 0.01 \\
\hline CCPG1 & 221511_PM_x_at & cell cycle progression 1 & 1.18 & 0.00 & 0.01 \\
\hline APC & 203527_PM_s_at & adenomatous polyposis coli & 1.18 & 0.00 & 0.01 \\
\hline--- & 244492_PM_at & --- & 1.18 & 0.00 & 0.01 \\
\hline MBOAT2 & 213288_PM_at & membrane bound O-acyltransferase domaiı & 1.18 & 0.00 & 0.01 \\
\hline ADAMTS2 & 226311_PM_at & ADAM metallopeptidase with thrombospon & 1.18 & 0.00 & 0.01 \\
\hline G6PD & 202275_PM_at & glucose-6-phosphate dehydrogenase & 1.18 & 0.00 & 0.01 \\
\hline--- & 226542_PM_at & --- & 1.18 & 0.00 & 0.01 \\
\hline MOSPD2 & 64883_PM_at & motile sperm domain containing 2 & 1.18 & 0.00 & 0.01 \\
\hline EIF2AK1 & 217735_PM_s_at & eukaryotic translation initiation factor 2 -alp & 1.18 & 0.00 & 0.01 \\
\hline CD36 & 206488_PM_s_at & CD36 molecule (thrombospondin receptor) & 1.18 & 0.00 & 0.0 \\
\hline
\end{tabular}




\begin{tabular}{|c|c|c|c|c|c|}
\hline ATP6V1C1 & 202872_PM_at & ATPase, $\mathrm{H}+$ transporting, lysosomal $42 \mathrm{kDa}$, ' & 1.18 & 0.00 & 0.02 \\
\hline PACSIN2 & 1554691_PM_a_at & protein kinase $C$ and casein kinase substrat & 1.18 & 0.00 & 0.02 \\
\hline SORD & 201563_PM_at & sorbitol dehydrogenase & 1.18 & 0.00 & 0.02 \\
\hline AMPD3 & 207992_PM_s_at & adenosine monophosphate deaminase 3 & 1.19 & 0.00 & 0.00 \\
\hline NSMAF & 203269_PM_at & neutral sphingomyelinase ( $\mathrm{N}$-SMase) activa & 1.19 & 0.00 & 0.00 \\
\hline SLCO3A1 & 227367_PM_at & solute carrier organic anion transporter farr & 1.19 & 0.00 & 0.00 \\
\hline--- & 242366_PM_at & --- & 1.19 & 0.00 & 0.00 \\
\hline LOC100509498 & 227290_PM_at & uncharacterized LOC100509498 & 1.19 & 0.00 & 0.00 \\
\hline--- & 229359_PM_at & --- & 1.19 & 0.00 & 0.00 \\
\hline S100A9 & 203535_PM_at & S100 calcium binding protein A9 & 1.19 & 0.00 & 0.00 \\
\hline PIWIL4 & 230480_PM_at & piwi-like 4 (Drosophila) & 1.19 & 0.00 & 0.00 \\
\hline NCF4 & 207677_PM_s_at & neutrophil cytosolic factor $4,40 \mathrm{kDa}$ & 1.19 & 0.00 & 0.00 \\
\hline sox6 & 227498_PM_at & SRY (sex determining region Y)-box 6 & 1.19 & 0.00 & 0.00 \\
\hline MEF2A & 212535_PM_at & myocyte enhancer factor $2 \mathrm{~A}$ & 1.19 & 0.00 & 0.00 \\
\hline TMEM167A & 224702_PM_at & transmembrane protein $167 \mathrm{~A}$ & 1.19 & 0.00 & 0.00 \\
\hline CR1 & 244313_PM_at & complement component (3b & 1.19 & 0.00 & 0.00 \\
\hline FAM45A & 225351_PM_at & family with sequence similarity 45, member & 1.19 & 0.00 & 0.00 \\
\hline ALOX5 & 213952_PM_s_at & Arachidonate 5-lipoxygenase & 1.19 & 0.00 & 0.00 \\
\hline ANTXR2 & 228573_PM_at & anthrax toxin receptor 2 & 1.19 & 0.00 & 0.00 \\
\hline C16orf72 & 1568954_PM_s_at & chromosome 16 open reading frame 72 & 1.19 & 0.00 & 0.01 \\
\hline TANK & 209451_PM_at & TRAF family member-associated NFKB activ & 1.19 & 0.00 & 0.01 \\
\hline RHOT1 & 218323_PM_at & ras homolog family member $\mathrm{T} 1$ & 1.19 & 0.00 & 0.01 \\
\hline--- & 244418_PM_at & --- & 1.19 & 0.00 & 0.01 \\
\hline KIAA0146 & 213003_PM_s_at & KIAA0146 & 1.19 & 0.00 & 0.01 \\
\hline C9orf66 & 1552755_PM_at & chromosome 9 open reading frame 66 & 1.19 & 0.00 & 0.01 \\
\hline CCDC71L & 229521_PM_at & coiled-coil domain containing 71-like & 1.19 & 0.00 & 0.02 \\
\hline DGAT2 & 226064_PM_s_at & diacylglycerol O-acyltransferase 2 & 1.19 & 0.00 & 0.02 \\
\hline C14orf2 & 202279_PM_at & chromosome 14 open reading frame 2 & 1.19 & 0.00 & 0.02 \\
\hline CYP4F3 & 206515_PM_at & cytochrome P450, family 4, subfamily F, pol & 1.19 & 0.00 & 0.02 \\
\hline MMRN1 & 205612_PM_at & multimerin 1 & 1.19 & 0.00 & 0.02 \\
\hline TRPM6 & 221102_PM_s_at & transient receptor potential cation channel, & 1.19 & 0.00 & 0.02 \\
\hline LTBR & 203005_PM_at & lymphotoxin beta receptor (TNFR superfam & 1.19 & 0.00 & 0.02 \\
\hline --- & 235759_PM_at & --- & 1.19 & 0.00 & 0.02 \\
\hline
\end{tabular}




\begin{tabular}{|c|c|c|c|c|c|}
\hline SORT1 & 224818_PM_at & sortilin 1 & 1.19 & 0.00 & 0.02 \\
\hline SAP30 & 204900_PM_x_at & Sin3A-associated protein, 30kDa & 1.19 & 0.00 & 0.02 \\
\hline F11R & 224097_PM_s_at & F11 receptor & 1.19 & 0.00 & 0.02 \\
\hline--- & 239560_PM_at & --- & 1.19 & 0.00 & 0.02 \\
\hline NT5C2 & 209155_PM_s_at & 5'-nucleotidase, cytosolic II & 1.2 & 0.00 & 0.00 \\
\hline MAPK1 & 212271_PM_at & mitogen-activated protein kinase 1 & 1.2 & 0.00 & 0.00 \\
\hline LYST & 203518_PM_at & lysosomal trafficking regulator & 1.2 & 0.00 & 0.00 \\
\hline ALOX5 & 204446_PM_s_at & arachidonate 5-lipoxygenase & 1.2 & 0.00 & 0.00 \\
\hline CFLAR & 237367_PM_x_at & CASP8 and FADD-like apoptosis regulator & 1.2 & 0.00 & 0.00 \\
\hline TMEM33 & 225492_PM_at & transmembrane protein 33 & 1.2 & 0.00 & 0.00 \\
\hline--- & 229968_PM_at & --- & 1.2 & 0.00 & 0.00 \\
\hline TLR8 & 229560_PM_at & toll-like receptor 8 & 1.2 & 0.00 & 0.00 \\
\hline KREMEN1 & 224534_PM_at & kringle containing transmembrane protein : & 1.2 & 0.00 & 0.00 \\
\hline C7orf25 & 53202_PM_at & chromosome 7 open reading frame 25 & 1.2 & 0.00 & 0.00 \\
\hline RAB27A & 209514_PM_s_at & RAB27A, member RAS oncogene family & 1.2 & 0.00 & 0.00 \\
\hline NCF4 & 205147_PM_x_at & neutrophil cytosolic factor $4,40 \mathrm{kDa}$ & 1.2 & 0.00 & 0.00 \\
\hline GYG1 & 201554_PM_x_at & glycogenin 1 & 1.2 & 0.00 & 0.00 \\
\hline CSF2RA & 210340_PM_s_at & colony stimulating factor 2 receptor, alpha, & 1.2 & 0.00 & 0.00 \\
\hline TRIQK & 225600_PM_at & triple QxxK & 1.2 & 0.00 & 0.00 \\
\hline TLR6 & 207446_PM_at & toll-like receptor 6 & 1.2 & 0.00 & 0.00 \\
\hline KIAA0141 & 227056_PM_at & KIAA0141 & 1.2 & 0.00 & 0.00 \\
\hline FAM217B & 225313_PM_at & family with sequence similarity 217, memb & 1.2 & 0.00 & 0.00 \\
\hline MAN2A2 & 219999_PM_at & mannosidase, alpha, class $2 \mathrm{~A}$, member 2 & 1.2 & 0.00 & 0.00 \\
\hline CDC14B & 221556_PM_at & CDC14 cell division cycle 14 homolog B (S. C & 1.2 & 0.00 & 0.01 \\
\hline CCPG1 & 222156_PM_x_at & cell cycle progression 1 & 1.2 & 0.00 & 0.01 \\
\hline CTBS & 218923_PM_at & chitobiase, di-N-acetyl- & 1.2 & 0.00 & 0.01 \\
\hline IFNGR1 & 211676_PM_s_at & interferon gamma receptor 1 & 1.2 & 0.00 & 0.01 \\
\hline CR1 & 208488_PM_s_at & complement component (3b & 1.2 & 0.00 & 0.01 \\
\hline CYYR1 & 228665_PM_at & cysteine & 1.2 & 0.00 & 0.01 \\
\hline--- & 238455_PM_at & --- & 1.2 & 0.00 & 0.01 \\
\hline UBL3 & 201535_PM_at & ubiquitin-like 3 & 1.2 & 0.00 & 0.01 \\
\hline KLHL8 & 242648_PM_at & kelch-like 8 (Drosophila) & 1.2 & 0.00 & 0.01 \\
\hline--- & 230176_PM_at & --- & 1.2 & 0.00 & 0.01 \\
\hline
\end{tabular}




\begin{tabular}{|c|c|c|c|c|c|}
\hline JAK2 & 205842_PM_s_at & Janus kinase 2 & 1.2 & 0.00 & 0.01 \\
\hline CHURC1 & 233268_PM_s_at & churchill domain containing 1 & 1.2 & 0.00 & 0.01 \\
\hline CENPW & 226936_PM_at & centromere protein $\mathrm{W}$ & 1.2 & 0.00 & 0.01 \\
\hline--- & 1560257_PM_at & --- & 1.2 & 0.00 & 0.02 \\
\hline LIMK2 & 210582_PM_s_at & LIM domain kinase 2 & 1.2 & 0.00 & 0.02 \\
\hline FAS & 204781_PM_s_at & Fas (TNF receptor superfamily, member 6) & 1.2 & 0.00 & 0.02 \\
\hline ST8SIA4 & 230261_PM_at & ST8 alpha-N-acetyl-neuraminide alpha-2,8-؟ & 1.2 & 0.00 & 0.02 \\
\hline F13A1 & 203305_PM_at & coagulation factor XIII, A1 polypeptide & 1.2 & 0.00 & 0.02 \\
\hline SRPK1 & 202199_PM_s_at & SRSF protein kinase 1 & 1.2 & 0.00 & 0.02 \\
\hline DNAJC3-AS1 & 240574_PM_at & DNAJC3 antisense RNA 1 (non-protein codir & 1.2 & 0.00 & 0.02 \\
\hline ROPN1L & 233312_PM_at & rhophilin associated tail protein 1-like & 1.2 & 0.00 & 0.02 \\
\hline APOBECЗА & 210873_PM_x_at & apolipoprotein B mRNA editing enzyme, cat & 1.2 & 0.00 & 0.03 \\
\hline ALAS1 & 205633_PM_s_at & aminolevulinate, delta-, synthase 1 & 1.21 & 0.00 & 0.00 \\
\hline PLXNC1 & 213241_PM_at & plexin C1 & 1.21 & 0.00 & 0.00 \\
\hline ENTPD1 & 209473_PM_at & ectonucleoside triphosphate diphosphohyd & 1.21 & 0.00 & 0.00 \\
\hline BHLHE40 & 201170_PM_s_at & basic helix-loop-helix family, member e40 & 1.21 & 0.00 & 0.00 \\
\hline ADAM8 & 205180_PM_s_at & ADAM metallopeptidase domain 8 & 1.21 & 0.00 & 0.00 \\
\hline HEBP2 & 203430_PM_at & heme binding protein 2 & 1.21 & 0.00 & 0.00 \\
\hline EGLN1 & 221497_PM_x_at & egI nine homolog 1 (C. elegans) & 1.21 & 0.00 & 0.00 \\
\hline UBE2J1 & 217825_PM_s_at & ubiquitin-conjugating enzyme E2, J1 & 1.21 & 0.00 & 0.00 \\
\hline--- & 1569568_PM_at & --- & 1.21 & 0.00 & 0.00 \\
\hline PXK & 225796_PM_at & PX domain containing serine & 1.21 & 0.00 & 0.00 \\
\hline CREBRF & 238476_PM_at & CREB3 regulatory factor & 1.21 & 0.00 & 0.00 \\
\hline TP53I3 & 210609_PM_s_at & tumor protein p53 inducible protein 3 & 1.21 & 0.00 & 0.00 \\
\hline CAT & 211922_PM_s_at & catalase & 1.21 & 0.00 & 0.00 \\
\hline CARD16 & 1552703_PM_s_at & caspase recruitment domain family, membe & 1.21 & 0.00 & 0.00 \\
\hline CNIH4 & 223993_PM_s_at & cornichon homolog 4 (Drosophila) & 1.21 & 0.00 & 0.00 \\
\hline FNDC3B & 225032_PM_at & fibronectin type III domain containing 3B & 1.21 & 0.00 & 0.00 \\
\hline QPCT & 205174_PM_s_at & glutaminyl-peptide cyclotransferase & 1.21 & 0.00 & 0.00 \\
\hline--- & 235898_PM_at & --- & 1.21 & 0.00 & 0.00 \\
\hline CPD & 201943_PM_s_at & carboxypeptidase D & 1.21 & 0.00 & 0.01 \\
\hline SLC12A6 & 223596_PM_at & solute carrier family 12 (potassium & 1.21 & 0.00 & 0.01 \\
\hline--- & 1569052_PM_at & --- & 1.21 & 0.00 & 0.01 \\
\hline
\end{tabular}




\begin{tabular}{|c|c|c|c|c|c|}
\hline JAG1 & 231183_PM_s_at & Jagged 1 & 1.21 & 0.00 & 0.01 \\
\hline ZNF578 & 1562245_PM_a_at & Zinc finger protein 578 & 1.21 & 0.00 & 0.01 \\
\hline LOC100128737 & 228753_PM_at & uncharacterized LOC100128737 & 1.21 & 0.00 & 0.01 \\
\hline MTMR6 & 214429_PM_at & myotubularin related protein 6 & 1.21 & 0.00 & 0.01 \\
\hline HDGFRP3 & 209526_PM_s_at & hepatoma-derived growth factor, related pı & 1.21 & 0.00 & 0.01 \\
\hline APC & 203525_PM_s_at & adenomatous polyposis coli & 1.21 & 0.00 & 0.01 \\
\hline DYSF & 218660_PM_at & dysferlin, limb girdle muscular dystrophy $2 \mathrm{E}$ & 1.21 & 0.00 & 0.01 \\
\hline CR1 & 206244_PM_at & complement component $(3 b$ & 1.21 & 0.00 & 0.01 \\
\hline ACPP & 204393_PM_s_at & acid phosphatase, prostate & 1.21 & 0.00 & 0.01 \\
\hline--- & 242094_PM_at & --- & 1.21 & 0.00 & 0.01 \\
\hline GLRX & 209276_PM_s_at & glutaredoxin (thioltransferase) & 1.21 & 0.00 & 0.01 \\
\hline TMEM88 & 229452_PM_at & transmembrane protein 88 & 1.21 & 0.00 & 0.02 \\
\hline FBXL13 & 1553798_PM_a_at & F-box and leucine-rich repeat protein 13 & 1.21 & 0.00 & 0.02 \\
\hline RAB27B & 228708_PM_at & RAB27B, member RAS oncogene family & 1.21 & 0.00 & 0.02 \\
\hline LPCAT2 & 227889_PM_at & Iysophosphatidylcholine acyltransferase 2 & 1.21 & 0.00 & 0.02 \\
\hline KCNE1 & 236407_PM_at & potassium voltage-gated channel, Isk-relate & 1.21 & 0.00 & 0.02 \\
\hline--- & 1569952_PM_x_at & --- & 1.21 & 0.00 & 0.02 \\
\hline DACH1 & 205471_PM_s_at & dachshund homolog 1 (Drosophila) & 1.21 & 0.00 & 0.03 \\
\hline ARG1 & 231663_PM_s_at & Arginase, liver & 1.21 & 0.00 & 0.03 \\
\hline CKLF & 219161_PM_s_at & chemokine-like factor & 1.22 & 0.00 & 0.00 \\
\hline CAT & 201432_PM_at & catalase & 1.22 & 0.00 & 0.00 \\
\hline S100A8 & 202917_PM_s_at & S100 calcium binding protein A8 & 1.22 & 0.00 & 0.00 \\
\hline KCTD21 & 229873_PM_at & potassium channel tetramerisation domain & 1.22 & 0.00 & 0.00 \\
\hline PGM2 & 225367_PM_at & phosphoglucomutase 2 & 1.22 & 0.00 & 0.00 \\
\hline СТВР2 & 201218_PM_at & C-terminal binding protein 2 & 1.22 & 0.00 & 0.00 \\
\hline DPH3 & 225200_PM_at & DPH3, KTI11 homolog (S. cerevisiae) & 1.22 & 0.00 & 0.00 \\
\hline SH3GLB1 & 210101_PM_x_at & SH3-domain GRB2-like endophilin B1 & 1.22 & 0.00 & 0.00 \\
\hline DRAM1 & 218627_PM_at & DNA-damage regulated autophagy modulat & 1.22 & 0.00 & 0.00 \\
\hline CEP350 & 213165_PM_at & centrosomal protein 350kDa & 1.22 & 0.00 & 0.00 \\
\hline RAB27A & 210951_PM_x_at & RAB27A, member RAS oncogene family & 1.22 & 0.00 & 0.00 \\
\hline ARHGAP26 & 226576_PM_at & Rho GTPase activating protein 26 & 1.22 & 0.00 & 0.00 \\
\hline ANKRD12 & 216563_PM_at & ankyrin repeat domain 12 & 1.22 & 0.00 & 0.00 \\
\hline--- & 227857_PM_at & --- & 1.22 & 0.00 & 0.00 \\
\hline
\end{tabular}




\begin{tabular}{|c|c|c|c|c|c|}
\hline ASAP1 & 224790_PM_at & ArfGAP with $\mathrm{SH} 3$ domain, ankyrin repeat an & 1.22 & 0.00 & 0.00 \\
\hline CREB5 & 229228_PM_at & CAMP responsive element binding protein 5 & 1.22 & 0.00 & 0.00 \\
\hline DPH3 & 225195_PM_at & DPH3, KTI11 homolog (S. cerevisiae) & 1.22 & 0.00 & 0.00 \\
\hline--- & 229373_PM_at & --- & 1.22 & 0.00 & 0.00 \\
\hline RRAGD & 221523_PM_s_at & Ras-related GTP binding D & 1.22 & 0.00 & 0.00 \\
\hline PLIN5 & 241368_PM_at & perilipin 5 & 1.22 & 0.00 & 0.00 \\
\hline--- & 242818_PM_x_at & --- & 1.22 & 0.00 & 0.01 \\
\hline CYSTM1 & 224707_PM_at & cysteine-rich transmembrane module cont $\bar{c}$ & 1.22 & 0.00 & 0.01 \\
\hline HSDL2 & 209512_PM_at & hydroxysteroid dehydrogenase like 2 & 1.22 & 0.00 & 0.01 \\
\hline CYBRD1 & 222453_PM_at & cytochrome $b$ reductase 1 & 1.22 & 0.00 & 0.01 \\
\hline HRASLS5 & 231050_PM_at & HRAS-like suppressor family, member 5 & 1.22 & 0.00 & 0.01 \\
\hline ASRGL1 & 222764_PM_at & asparaginase like 1 & 1.22 & 0.00 & 0.01 \\
\hline ST3GAL6 & 210942_PM_s_at & ST3 beta-galactoside alpha-2,3-sialyltransfe & 1.22 & 0.00 & 0.02 \\
\hline FLG-AS1 & 1570423_PM_at & FLG antisense RNA 1 (non-protein coding) & 1.22 & 0.00 & 0.02 \\
\hline LRG1 & 228648_PM_at & leucine-rich alpha-2-glycoprotein 1 & 1.22 & 0.00 & 0.02 \\
\hline ATP8B4 & 220416_PM_at & ATPase, class I, type 8B, member 4 & 1.22 & 0.00 & 0.02 \\
\hline HIST1H2BC & 214455_PM_at & histone cluster $1, \mathrm{H} 2 \mathrm{bc}$ & 1.22 & 0.00 & 0.03 \\
\hline ALPL & 215783_PM_s_at & alkaline phosphatase, liver & 1.22 & 0.00 & 0.03 \\
\hline NDFIP2 & 224802_PM_at & Nedd4 family interacting protein 2 & 1.22 & 0.00 & 0.03 \\
\hline STS & 203767_PM_s_at & steroid sulfatase (microsomal), isozyme $S$ & 1.22 & 0.00 & 0.03 \\
\hline RAB32 & 228161_PM_at & RAB32, member RAS oncogene family & 1.23 & 0.00 & 0.00 \\
\hline MYO5A & 204527_PM_at & myosin VA (heavy chain 12 , myoxin) & 1.23 & 0.00 & 0.00 \\
\hline CARD6 & 224414_PM_s_at & caspase recruitment domain family, memb & 1.23 & 0.00 & 0.00 \\
\hline SH3GLB1 & 209091_PM_s_at & SH3-domain GRB2-like endophilin B1 & 1.23 & 0.00 & 0.00 \\
\hline $\mathrm{RHOQ}$ & 212120_PM_at & ras homolog family member $\mathrm{Q}$ & 1.23 & 0.00 & 0.00 \\
\hline--- & 240673_PM_at & --- & 1.23 & 0.00 & 0.00 \\
\hline IL18 & 206295_PM_at & interleukin 18 (interferon-gamma-inducing & 1.23 & 0.00 & 0.00 \\
\hline DCBLD2 & 230175_PM_s_at & Discoidin, CUB and LCCL domain containing & 1.23 & 0.00 & 0.00 \\
\hline MGC70870 & 242136_PM_x_at & C-terminal binding protein 2 pseudogene & 1.23 & 0.00 & 0.00 \\
\hline LILRB2 & 210146_PM_x_at & leukocyte immunoglobulin-like receptor, su & 1.23 & 0.00 & 0.00 \\
\hline TP53І11 & 203421_PM_at & tumor protein p53 inducible protein 11 & 1.23 & 0.00 & 0.00 \\
\hline HES1 & 203394_PM_s_at & hairy and enhancer of split 1, (Drosophila) & 1.23 & 0.00 & 0.00 \\
\hline MGST1 & 1565162_PM_s_at & microsomal glutathione S-transferase 1 & 1.23 & 0.00 & 0.00 \\
\hline
\end{tabular}




\begin{tabular}{|c|c|c|c|c|c|}
\hline PIK3R6 & 1558770_PM_a_at & phosphoinositide-3-kinase, regulatory subu & 1.23 & 0.00 & 0.01 \\
\hline MRVI1 & 226047_PM_at & murine retrovirus integration site 1 homolo & 1.23 & 0.00 & 0.01 \\
\hline--- & 237904_PM_at & --- & 1.23 & 0.00 & 0.01 \\
\hline CSTA & 204971_PM_at & cystatin A (stefin A) & 1.23 & 0.00 & 0.01 \\
\hline PALLD & 200897_PM_s_at & palladin, cytoskeletal associated protein & 1.23 & 0.00 & 0.01 \\
\hline $\mathrm{CNIH} 4$ & 218728_PM_s_at & cornichon homolog 4 (Drosophila) & 1.23 & 0.00 & 0.01 \\
\hline--- & 239108_PM_at & --- & 1.23 & 0.00 & 0.01 \\
\hline SLC22A1 & 207201_PM_s_at & solute carrier family 22 (organic cation tran: & 1.23 & 0.00 & 0.01 \\
\hline ST6GALNAC3 & 235334_PM_at & ST6 (alpha-N-acetyl-neuraminyl-2,3-beta-ga & 1.23 & 0.00 & 0.02 \\
\hline NRG1 & 206237_PM_s_at & neuregulin 1 & 1.23 & 0.00 & 0.02 \\
\hline EXOC8 & 227577_PM_at & exocyst complex component 8 & 1.23 & 0.00 & 0.03 \\
\hline CHST15 & 244874_PM_at & carbohydrate ( $\mathrm{N}$-acetylgalactosamine 4-sulf & 1.23 & 0.00 & 0.03 \\
\hline GUSBP2 & 206565_PM_x_at & glucuronidase, beta pseudogene 2 & 1.23 & 0.00 & 0.03 \\
\hline C18orf32 & 224957_PM_at & chromosome 18 open reading frame 32 & 1.23 & 0.00 & 0.03 \\
\hline LGALS12 & 223828_PM_s_at & lectin, galactoside-binding, soluble, 12 & 1.23 & 0.00 & 0.03 \\
\hline LOC100653002 & 231950_PM_at & zinc finger protein 658-like & 1.23 & 0.00 & 0.05 \\
\hline--- & 239294_PM_at & --- & 1.24 & 0.00 & 0.00 \\
\hline UBE2J1 & 222435_PM_s_at & ubiquitin-conjugating enzyme E2, J1 & 1.24 & 0.00 & 0.00 \\
\hline HK3 & 205936_PM_s_at & hexokinase 3 (white cell) & 1.24 & 0.00 & 0.00 \\
\hline MEGF9 & 212830_PM_at & multiple EGF-like-domains 9 & 1.24 & 0.00 & 0.00 \\
\hline--- & 240020_PM_at & --- & 1.24 & 0.00 & 0.00 \\
\hline MIR4500HG & 1569348_PM_at & MIR4500 host gene (non-protein coding) & 1.24 & 0.00 & 0.00 \\
\hline PIP5K1B & 205632_PM_s_at & phosphatidylinositol-4-phosphate 5-kinase, & 1.24 & 0.00 & 0.00 \\
\hline PDZD8 & 225829_PM_at & PDZ domain containing 8 & 1.24 & 0.00 & 0.00 \\
\hline RNF141 & 226106_PM_at & ring finger protein 141 & 1.24 & 0.00 & 0.00 \\
\hline HIPK3 & 210148_PM_at & homeodomain interacting protein kinase 3 & 1.24 & 0.00 & 0.00 \\
\hline LOC100509635 & 226865_PM_at & uncharacterized LOC100509635 & 1.24 & 0.00 & 0.00 \\
\hline ROPN1L & 223609_PM_at & rhophilin associated tail protein 1-like & 1.24 & 0.00 & 0.00 \\
\hline MFSD9 & 213393_PM_at & major facilitator superfamily domain contai & 1.24 & 0.00 & 0.00 \\
\hline ACSL4 & 202422_PM_s_at & acyl-CoA synthetase long-chain family mem & 1.24 & 0.00 & 0.00 \\
\hline AZU1 & 214575_PM_s_at & azurocidin 1 & 1.24 & 0.00 & 0.00 \\
\hline EMR2 & 207610_PM_s_at & egf-like module containing, mucin-like, horr & 1.24 & 0.00 & 0.01 \\
\hline FAM198B & 223204_PM_at & family with sequence similarity 198, membs & 1.24 & 0.00 & 0.01 \\
\hline
\end{tabular}




\begin{tabular}{|c|c|c|c|c|c|}
\hline KRCC1 & 233329_PM_s_at & lysine-rich coiled-coil 1 & 1.24 & 0.00 & 0.01 \\
\hline B3GNT5 & 225612_PM_s_at & UDP-GIcNAc:betaGal beta-1,3-N-acetylglucc & 1.24 & 0.00 & 0.01 \\
\hline BCL6 & 215990_PM_s_at & B-cell CLL & 1.24 & 0.00 & 0.01 \\
\hline CA4 & 206209_PM_s_at & carbonic anhydrase IV & 1.24 & 0.00 & 0.02 \\
\hline DEFB106A & 1552411_PM_at & defensin, beta $106 \mathrm{~A}$ & 1.24 & 0.00 & 0.02 \\
\hline CYP4F2 & 206514_PM_s_at & cytochrome P450, family 4 , subfamily F, pol & 1.24 & 0.00 & 0.02 \\
\hline ABCA1 & 203505_PM_at & ATP-binding cassette, sub-family $A(A B C 1)$, I & 1.24 & 0.00 & 0.02 \\
\hline GRB10 & 215248_PM_at & growth factor receptor-bound protein 10 & 1.24 & 0.00 & 0.02 \\
\hline IRAK3 & 213817_PM_at & interleukin-1 receptor-associated kinase 3 & 1.24 & 0.00 & 0.02 \\
\hline CD9 & 201005_PM_at & CD9 molecule & 1.24 & 0.00 & 0.02 \\
\hline ITGA9 & 227297_PM_at & integrin, alpha 9 & 1.24 & 0.00 & 0.02 \\
\hline IQGAP3 & 229538_PM_s_at & IQ motif containing GTPase activating prote & 1.24 & 0.00 & 0.03 \\
\hline CYBRD1 & 217889_PM_s_at & cytochrome $b$ reductase 1 & 1.24 & 0.00 & 0.03 \\
\hline COL9A2 & 213622_PM_at & collagen, type IX, alpha 2 & 1.24 & 0.00 & 0.03 \\
\hline RAB33B & 221014_PM_s_at & RAB33B, member RAS oncogene family & 1.24 & 0.00 & 0.04 \\
\hline HECW2 & 232080_PM_at & $\mathrm{HECT}, \mathrm{C} 2$ and WW domain containing E3 ub & 1.24 & 0.00 & 0.04 \\
\hline SORT1 & 212807_PM_s_at & sortilin 1 & 1.25 & 0.00 & 0.00 \\
\hline TLR2 & 204924_PM_at & toll-like receptor 2 & 1.25 & 0.00 & 0.00 \\
\hline--- & 239117_PM_at & --- & 1.25 & 0.00 & 0.00 \\
\hline C3AR1 & 209906_PM_at & complement component 3a receptor 1 & 1.25 & 0.00 & 0.00 \\
\hline ARSB & 232197_PM_x_at & arylsulfatase B & 1.25 & 0.00 & 0.00 \\
\hline RNF175 & 236465_PM_at & ring finger protein 175 & 1.25 & 0.00 & 0.00 \\
\hline UBE2J1 & 217823_PM_s_at & ubiquitin-conjugating enzyme E2, J1 & 1.25 & 0.00 & 0.00 \\
\hline NUDT16P1 & 232251_PM_at & nudix (nucleoside diphosphate linked moiet & 1.25 & 0.00 & 0.00 \\
\hline DEFT1P & 1553518_PM_at & defensin, theta 1 pseudogene & 1.25 & 0.00 & 0.00 \\
\hline SLC22A4 & 205896_PM_at & solute carrier family 22 (organic cation & 1.25 & 0.00 & 0.00 \\
\hline WDFY3 & 212598_PM_at & WD repeat and FYVE domain containing 3 & 1.25 & 0.00 & 0.00 \\
\hline ACSL1 & 207275_PM_s_at & acyl-CoA synthetase long-chain family mem & 1.25 & 0.00 & 0.00 \\
\hline WDFY3 & 212602_PM_at & WD repeat and FYVE domain containing 3 & 1.25 & 0.00 & 0.00 \\
\hline LPCAT2 & 222833_PM_at & Iysophosphatidylcholine acyltransferase 2 & 1.25 & 0.00 & 0.01 \\
\hline LOC100505702 & 229635_PM_at & uncharacterized LOC100505702 & 1.25 & 0.00 & 0.01 \\
\hline--- & 1570301_PM_at & --- & 1.25 & 0.00 & 0.01 \\
\hline ACPP & 231711_PM_at & Acid phosphatase, prostate & 1.25 & 0.00 & 0.01 \\
\hline
\end{tabular}




\begin{tabular}{|c|c|c|c|c|c|}
\hline BEND2 & 1553842_PM_at & BEN domain containing 2 & 1.25 & 0.00 & 0.01 \\
\hline ZNF608 & 229817_PM_at & zinc finger protein 608 & 1.25 & 0.00 & 0.01 \\
\hline FAM124B & 220637_PM_at & family with sequence similarity $124 \mathrm{~B}$ & 1.25 & 0.00 & 0.01 \\
\hline 1-Mar & 218865_PM_at & mitochondrial amidoxime reducing compon & 1.25 & 0.00 & 0.01 \\
\hline $\mathrm{PI3}$ & 203691_PM_at & peptidase inhibitor 3, skin-derived & 1.25 & 0.00 & 0.02 \\
\hline CHST13 & 239647_PM_at & carbohydrate (chondroitin 4) sulfotransfera & 1.25 & 0.00 & 0.02 \\
\hline CD163 & 216233_PM_at & CD163 molecule & 1.25 & 0.00 & 0.02 \\
\hline SLC5A9 & 232378_PM_at & solute carrier family 5 (sodium & 1.25 & 0.00 & 0.02 \\
\hline SPP1 & 1568574_PM_x_at & Secreted phosphoprotein 1 & 1.25 & 0.00 & 0.03 \\
\hline HNMT & 204112_PM_s_at & histamine $\mathrm{N}$-methyltransferase & 1.25 & 0.00 & 0.03 \\
\hline PFKFB2 & 226733_PM_at & 6-phosphofructo-2-kinase & 1.25 & 0.00 & 0.03 \\
\hline RPS27L & 222487_PM_s_at & ribosomal protein S27-like & 1.25 & 0.00 & 0.04 \\
\hline PYGL & 202990_PM_at & phosphorylase, glycogen, liver & 1.26 & 0.00 & 0.00 \\
\hline TSNAX & 238973_PM_s_at & translin-associated factor $X$ & 1.26 & 0.00 & 0.00 \\
\hline RAB13 & 202252_PM_at & RAB13, member RAS oncogene family & 1.26 & 0.00 & 0.00 \\
\hline--- & 237563_PM_s_at & --- & 1.26 & 0.00 & 0.00 \\
\hline--- & 235286_PM_at & --- & 1.26 & 0.00 & 0.00 \\
\hline WDFY3 & 212606_PM_at & WD repeat and FYVE domain containing 3 & 1.26 & 0.00 & 0.00 \\
\hline JAG1 & 209099_PM_x_at & jagged 1 & 1.26 & 0.00 & 0.00 \\
\hline PLBD1 & 222639_PM_s_at & phospholipase B domain containing 1 & 1.26 & 0.00 & 0.00 \\
\hline FAM120A & 1555947_PM_at & family with sequence similarity $120 \mathrm{~A}$ & 1.26 & 0.00 & 0.00 \\
\hline SNORD89 & 235536_PM_at & small nucleolar RNA, C & 1.26 & 0.00 & 0.00 \\
\hline NDUFB3 & 203371_PM_s_at & $\mathrm{NADH}$ dehydrogenase (ubiquinone) 1 beta & 1.26 & 0.00 & 0.00 \\
\hline ITPR2 & 202660_PM_at & inositol 1,4,5-trisphosphate receptor, type: & 1.26 & 0.00 & 0.00 \\
\hline--- & 207596_PM_at & --- & 1.26 & 0.00 & 0.00 \\
\hline TRPM6 & 224412_PM_s_at & transient receptor potential cation channel, & 1.26 & 0.00 & 0.00 \\
\hline LIN7A & 206440_PM_at & lin-7 homolog A (C. elegans) & 1.26 & 0.00 & 0.00 \\
\hline OSBPL1A & 208158_PM_s_at & oxysterol binding protein-like $1 \mathrm{~A}$ & 1.26 & 0.00 & 0.00 \\
\hline SLC29A1 & 201801_PM_s_at & solute carrier family 29 (nucleoside transpo & 1.26 & 0.00 & 0.00 \\
\hline SLC24A3 & 219090_PM_at & solute carrier family 24 (sodium & 1.26 & 0.00 & 0.00 \\
\hline APOBR & 220023_PM_at & apolipoprotein B receptor & 1.26 & 0.00 & 0.00 \\
\hline SLCO4C1 & 222071_PM_s_at & solute carrier organic anion transporter far & 1.26 & 0.00 & 0.00 \\
\hline IRAK3 & 220034_PM_at & interleukin-1 receptor-associated kinase 3 & 1.26 & 0.00 & 0.00 \\
\hline
\end{tabular}




\begin{tabular}{|c|c|c|c|c|c|}
\hline RGS12 & 1555032_PM_at & regulator of G-protein signaling 12 & 1.26 & 0.00 & 0.00 \\
\hline FSTL1 & 208782_PM_at & follistatin-like 1 & 1.26 & 0.00 & 0.00 \\
\hline ENTPD1 & 228585_PM_at & ectonucleoside triphosphate diphosphohyd & 1.26 & 0.00 & 0.01 \\
\hline SSFA2 & 229744_PM_at & sperm specific antigen 2 & 1.26 & 0.00 & 0.01 \\
\hline THBS1 & 235086_PM_at & thrombospondin 1 & 1.26 & 0.00 & 0.01 \\
\hline KCNJ15 & 238428_PM_at & potassium inwardly-rectifying channel, subf & 1.26 & 0.00 & 0.01 \\
\hline ASPH & 225008_PM_at & aspartate beta-hydroxylase & 1.26 & 0.00 & 0.03 \\
\hline S100A8 & 214370_PM_at & S100 calcium binding protein A8 & 1.27 & 0.00 & 0.00 \\
\hline RRAGD & 221524_PM_s_at & Ras-related GTP binding D & 1.27 & 0.00 & 0.00 \\
\hline CR1 & 217552_PM_x_at & complement component $(3 \mathrm{~b}$ & 1.27 & 0.00 & 0.00 \\
\hline CYP4F12 & 206539_PM_s_at & cytochrome P450, family 4, subfamily F, pol & 1.27 & 0.00 & 0.00 \\
\hline NFIL3 & 203574_PM_at & nuclear factor, interleukin 3 regulated & 1.27 & 0.00 & 0.00 \\
\hline TLR5 & 210166_PM_at & toll-like receptor 5 & 1.27 & 0.00 & 0.00 \\
\hline CD163 & 215049_PM_x_at & CD163 molecule & 1.27 & 0.00 & 0.00 \\
\hline LTB4R & 236172_PM_at & leukotriene B4 receptor & 1.27 & 0.00 & 0.00 \\
\hline JAG1 & 216268_PM_s_at & jagged 1 & 1.27 & 0.00 & 0.00 \\
\hline IKBIP & 227295_PM_at & IKBKB interacting protein & 1.27 & 0.00 & 0.00 \\
\hline CD163 & 203645_PM_s_at & CD163 molecule & 1.27 & 0.00 & 0.00 \\
\hline GPR84 & 223767_PM_at & G protein-coupled receptor 84 & 1.27 & 0.00 & 0.00 \\
\hline SLC37A3 & 223304_PM_at & solute carrier family 37 (glycerol-3-phospha & 1.27 & 0.00 & 0.00 \\
\hline SULT1B1 & 207601_PM_at & sulfotransferase family, cytosolic, 1B, meml & 1.27 & 0.00 & 0.01 \\
\hline--- & 239825_PM_at & --- & 1.27 & 0.00 & 0.01 \\
\hline ABCA1 & 203504_PM_s_at & ATP-binding cassette, sub-family $A(A B C 1)$, I & 1.27 & 0.00 & 0.01 \\
\hline $\mathrm{PI3}$ & 41469_PM_at & peptidase inhibitor 3 , skin-derived & 1.27 & 0.00 & 0.01 \\
\hline ZNF321P & 1558887_PM_at & zinc finger protein 321 , pseudogene & 1.27 & 0.00 & 0.01 \\
\hline ITGB3 & 204628_PM_s_at & integrin, beta 3 (platelet glycoprotein IIla, a & 1.27 & 0.00 & 0.02 \\
\hline RPS27L & 218007_PM_s_at & ribosomal protein S27-like & 1.27 & 0.00 & 0.03 \\
\hline $\mathrm{FCHO} 2$ & 228220_PM_at & FCH domain only 2 & 1.27 & 0.00 & 0.03 \\
\hline LPAR1 & 204036_PM_at & lysophosphatidic acid receptor 1 & 1.27 & 0.00 & 0.04 \\
\hline CALD1 & 212077_PM_at & caldesmon 1 & 1.27 & 0.00 & 0.04 \\
\hline DOCK4 & 205003_PM_at & dedicator of cytokinesis 4 & 1.27 & 0.00 & 0.05 \\
\hline ACOT11 & 216103_PM_at & acyl-CoA thioesterase 11 & 1.28 & 0.00 & 0.00 \\
\hline--- & 237071_PM_at & --- & 1.28 & 0.00 & 0.00 \\
\hline
\end{tabular}




\begin{tabular}{|c|c|c|c|c|c|}
\hline CCPG1 & 221156_PM_x_at & cell cycle progression 1 & 1.28 & 0.00 & 0.00 \\
\hline LIN7A & 240027_PM_at & lin-7 homolog A (C. elegans) & 1.28 & 0.00 & 0.00 \\
\hline F5 & 204713_PM_s_at & coagulation factor $\mathrm{V}$ (proaccelerin, labile fac & 1.28 & 0.00 & 0.00 \\
\hline SAMSN1 & 1555638_PM_a_at & SAM domain, SH3 domain and nuclear local & 1.28 & 0.00 & 0.00 \\
\hline PADI4 & 220001_PM_at & peptidyl arginine deiminase, type IV & 1.28 & 0.00 & 0.01 \\
\hline IGHA1 & 1558438_PM_a_at & Immunoglobulin heavy constant alpha 1 & 1.28 & 0.00 & 0.01 \\
\hline PGLYRP1 & 207384_PM_at & peptidoglycan recognition protein 1 & 1.28 & 0.00 & 0.01 \\
\hline--- & 230416_PM_at & --- & 1.28 & 0.00 & 0.01 \\
\hline YOD1 & 227309_PM_at & YOD1 OTU deubiquinating enzyme 1 homol & 1.28 & 0.00 & 0.02 \\
\hline CCDC126 & 228087_PM_at & coiled-coil domain containing 126 & 1.28 & 0.00 & 0.02 \\
\hline MYL9 & 201058_PM_s_at & myosin, light chain 9, regulatory & 1.28 & 0.00 & 0.02 \\
\hline FCGR1A & 216950_PM_s_at & Fc fragment of IgG, high affinity la, receptor & 1.28 & 0.00 & 0.02 \\
\hline IPMK & 238739_PM_at & inositol polyphosphate multikinase & 1.28 & 0.00 & 0.03 \\
\hline TRIQK & 225599_PM_s_at & triple QxxK & 1.28 & 0.00 & 0.04 \\
\hline IP011 & 238488_PM_at & importin 11 & 1.28 & 0.00 & 0.04 \\
\hline HIST1H3E & 236278_PM_at & Histone cluster $1, \mathrm{H} 3 \mathrm{e}$ & 1.28 & 0.00 & 0.04 \\
\hline HCAR3 & 205220_PM_at & hydroxycarboxylic acid receptor 3 & 1.29 & 0.00 & 0.00 \\
\hline LOC100507015 & 227356_PM_at & uncharacterized LOC100507015 & 1.29 & 0.00 & 0.00 \\
\hline ST3GAL6 & 213355_PM_at & ST3 beta-galactoside alpha-2,3-sialyltransfe & 1.29 & 0.00 & 0.00 \\
\hline C10orf128 & 228372_PM_at & chromosome 10 open reading frame 128 & 1.29 & 0.00 & 0.00 \\
\hline--- & 236297_PM_at & --- & 1.29 & 0.00 & 0.00 \\
\hline PLOD2 & 202620_PM_s_at & procollagen-lysine, 2-oxoglutarate 5-dioxyg & 1.29 & 0.00 & 0.00 \\
\hline CYP4F2 & 210452_PM_x_at & cytochrome P450, family 4, subfamily F, pol & 1.29 & 0.00 & 0.00 \\
\hline--- & 236898_PM_at & --- & 1.29 & 0.00 & 0.01 \\
\hline FCGR1B & 214511_PM_x_at & Fc fragment of IgG, high affinity Ib, receptoI & 1.29 & 0.00 & 0.01 \\
\hline FCGR1A & 216951_PM_at & Fc fragment of IgG, high affinity la, receptor & 1.29 & 0.00 & 0.02 \\
\hline SLC29A1 & 201802_PM_at & solute carrier family 29 (nucleoside transpo & 1.29 & 0.00 & 0.03 \\
\hline--- & 231672_PM_at & --- & 1.29 & 0.00 & 0.03 \\
\hline ACSL1 & 201963_PM_at & acyl-CoA synthetase long-chain family mem & 1.3 & 0.00 & 0.00 \\
\hline--- & 230778_PM_at & --- & 1.3 & 0.00 & 0.00 \\
\hline JAK2 & 205841_PM_at & Janus kinase 2 & 1.3 & 0.00 & 0.00 \\
\hline--- & 1567101_PM_at & --- & 1.3 & 0.00 & 0.00 \\
\hline FAM200B & 227270_PM_at & family with sequence similarity 200, membs & 1.3 & 0.00 & 0.00 \\
\hline
\end{tabular}




\begin{tabular}{|c|c|c|c|c|c|}
\hline ASPH & 224996_PM_at & aspartate beta-hydroxylase & 1.3 & 0.00 & 0.02 \\
\hline STXBP5 & 226794_PM_at & syntaxin binding protein 5 (tomosyn) & 1.31 & 0.00 & 0.00 \\
\hline MCTP2 & 229021_PM_at & multiple $\mathrm{C} 2$ domains, transmembrane 2 & 1.31 & 0.00 & 0.00 \\
\hline MYCT1 & 231947_PM_at & myc target 1 & 1.31 & 0.00 & 0.00 \\
\hline--- & 243824_PM_at & --- & 1.31 & 0.00 & 0.00 \\
\hline SGMS1 & 212989_PM_at & sphingomyelin synthase 1 & 1.31 & 0.00 & 0.00 \\
\hline GPR34 & 223620_PM_at & G protein-coupled receptor 34 & 1.31 & 0.00 & 0.00 \\
\hline CLEC5A & 219890_PM_at & C-type lectin domain family 5 , member $A$ & 1.31 & 0.00 & 0.01 \\
\hline $\mathrm{BMX}$ & 206464_PM_at & BMX non-receptor tyrosine kinase & 1.31 & 0.00 & 0.01 \\
\hline CD24 & 208651_PM_x_at & CD24 molecule & 1.31 & 0.00 & 0.01 \\
\hline MCTP2 & 220603_PM_s_at & multiple C2 domains, transmembrane 2 & 1.32 & 0.00 & 0.00 \\
\hline RETN & 220570_PM_at & resistin & 1.32 & 0.00 & 0.00 \\
\hline SAMSN1 & 220330_PM_s_at & SAM domain, SH3 domain and nuclear local & 1.32 & 0.00 & 0.00 \\
\hline $\mathrm{CD} 24$ & 209771_PM_x_at & CD24 molecule & 1.32 & 0.00 & 0.01 \\
\hline IL1R2 & 205403_PM_at & interleukin 1 receptor, type II & 1.32 & 0.00 & 0.01 \\
\hline PRUNE2 & 212805_PM_at & prune homolog 2 (Drosophila) & 1.32 & 0.00 & 0.02 \\
\hline COMMD8 & 218351_PM_at & COMM domain containing 8 & 1.32 & 0.00 & 0.02 \\
\hline BPGM & 203502_PM_at & 2,3-bisphosphoglycerate mutase & 1.32 & 0.00 & 0.04 \\
\hline HTATSF1P2 & 1558882_PM_at & HIV-1 Tat specific factor 1 pseudogene 2 & 1.32 & 0.00 & 0.04 \\
\hline SLC24A3 & 57588_PM_at & solute carrier family 24 (sodium & 1.33 & 0.00 & 0.00 \\
\hline CARD16 & 1552701_PM_a_at & caspase recruitment domain family, membe & 1.33 & 0.00 & 0.00 \\
\hline SYNE1 & 232027_PM_at & spectrin repeat containing, nuclear envelop & 1.33 & 0.00 & 0.00 \\
\hline CD24 & 216379_PM_x_at & CD24 molecule & 1.33 & 0.00 & 0.00 \\
\hline SMPD3 & 219695_PM_at & sphingomyelin phosphodiesterase 3 , neutra & 1.33 & 0.00 & 0.01 \\
\hline ECHDC3 & 219298_PM_at & enoyl CoA hydratase domain containing 3 & 1.33 & 0.00 & 0.02 \\
\hline PROS1 & 207808_PM_s_at & protein S (alpha) & 1.33 & 0.00 & 0.02 \\
\hline KIF1B & 225878_PM_at & kinesin family member $1 \mathrm{~B}$ & 1.34 & 0.00 & 0.00 \\
\hline LIN7A & 227929_PM_at & lin-7 homolog A (C. elegans) & 1.34 & 0.00 & 0.00 \\
\hline MSRB3 & 225782_PM_at & methionine sulfoxide reductase $\mathrm{B} 3$ & 1.34 & 0.00 & 0.00 \\
\hline EPAS1 & 200878_PM_at & endothelial PAS domain protein 1 & 1.34 & 0.00 & 0.00 \\
\hline DSC2 & 204751_PM_x_at & desmocollin 2 & 1.34 & 0.00 & 0.00 \\
\hline KREMEN1 & 227250_PM_at & kringle containing transmembrane protein & 1.34 & 0.00 & 0.01 \\
\hline OLIG1 & 228170_PM_at & oligodendrocyte transcription factor 1 & 1.34 & 0.00 & 0.01 \\
\hline
\end{tabular}




\begin{tabular}{|c|c|c|c|c|c|}
\hline ORM1 & 214465_PM_at & orosomucoid 1 & 1.34 & 0.00 & 0.01 \\
\hline LY96 & 206584_PM_at & Iymphocyte antigen 96 & 1.34 & 0.00 & 0.01 \\
\hline BCL2A1 & 205681_PM_at & $\mathrm{BCL2}$-related protein A1 & 1.34 & 0.00 & 0.02 \\
\hline ANKRD22 & 238439_PM_at & ankyrin repeat domain 22 & 1.34 & 0.00 & 0.02 \\
\hline TPST1 & 204140_PM_at & tyrosylprotein sulfotransferase 1 & 1.34 & 0.00 & 0.03 \\
\hline PNPLA1 & 1553364_PM_at & patatin-like phospholipase domain containi & 1.35 & 0.00 & 0.00 \\
\hline TRPM6 & 240389_PM_at & Transient receptor potential cation channel & 1.35 & 0.00 & 0.00 \\
\hline--- & 1567100_PM_at & --- & 1.35 & 0.00 & 0.00 \\
\hline MPO & 203948_PM_s_at & myeloperoxidase & 1.35 & 0.00 & 0.00 \\
\hline--- & 243252_PM_at & --- & 1.35 & 0.00 & 0.01 \\
\hline GAPT & 1552386_PM_at & GRB2-binding adaptor protein, transmembr & 1.36 & 0.00 & 0.00 \\
\hline DAPK2 & 215184_PM_at & death-associated protein kinase 2 & 1.36 & 0.00 & 0.00 \\
\hline NFE4 & 1560527_PM_at & transcription factor NF-E4 & 1.36 & 0.00 & 0.00 \\
\hline CYP1B1-AS1 & 1553829_PM_at & CYP1B1 antisense RNA 1 (non-protein codin & 1.36 & 0.00 & 0.00 \\
\hline SLC8A1 & 1556583_PM_a_at & solute carrier family 8 (sodium & 1.36 & 0.00 & 0.00 \\
\hline--- & 217329_PM_x_at & --- & 1.36 & 0.00 & 0.01 \\
\hline CYP1B1 & 202437_PM_s_at & cytochrome P450, family 1 , subfamily $B$, pol & 1.37 & 0.00 & 0.00 \\
\hline DSC2 & 226817_PM_at & desmocollin 2 & 1.37 & 0.00 & 0.01 \\
\hline MMP9 & 203936_PM_s_at & matrix metallopeptidase 9 (gelatinase B, 92 & 1.37 & 0.00 & 0.01 \\
\hline THBS1 & 201110_PM_s_at & thrombospondin 1 & 1.37 & 0.00 & 0.01 \\
\hline MOCS1 & 1555127_PM_at & molybdenum cofactor synthesis 1 & 1.37 & 0.00 & 0.02 \\
\hline ITGA2B & 206493_PM_at & integrin, alpha $2 \mathrm{~b}$ (platelet glycoprotein IIb & 1.38 & 0.00 & 0.00 \\
\hline PFKFB2 & 209992_PM_at & 6-phosphofructo-2-kinase & 1.38 & 0.00 & 0.00 \\
\hline--- & 240131_PM_at & --- & 1.38 & 0.00 & 0.00 \\
\hline SIGLEC8 & 208253_PM_at & sialic acid binding Ig-like lectin 8 & 1.38 & 0.00 & 0.04 \\
\hline CD24 & 209772_PM_s_at & CD24 molecule & 1.39 & 0.00 & 0.00 \\
\hline ELANE & 206871_PM_at & elastase, neutrophil expressed & 1.39 & 0.00 & 0.00 \\
\hline OLAH & 219975_PM_x_at & oleoyl-ACP hydrolase & 1.39 & 0.00 & 0.00 \\
\hline EVI2A & 204774_PM_at & ecotropic viral integration site $2 \mathrm{~A}$ & 1.39 & 0.00 & 0.01 \\
\hline C19orf59 & 235568_PM_at & chromosome 19 open reading frame 59 & 1.4 & 0.00 & 0.00 \\
\hline S100A12 & 205863_PM_at & S100 calcium binding protein A12 & 1.4 & 0.00 & 0.00 \\
\hline OLR1 & 210004_PM_at & oxidized low density lipoprotein (lectin-like) & 1.4 & 0.00 & 0.00 \\
\hline IL5RA & 210744_PM_s_at & interleukin 5 receptor, alpha & 1.4 & 0.00 & 0.01 \\
\hline
\end{tabular}




\begin{tabular}{|c|c|c|c|c|c|}
\hline F5 & 204714_PM_s_at & coagulation factor $\mathrm{V}$ (proaccelerin, labile fac & 1.41 & 0.00 & 0.00 \\
\hline PPBP & 214146_PM_s_at & pro-platelet basic protein (chemokine (C-X-1 & 1.41 & 0.00 & 0.00 \\
\hline VSTM1 & 235818_PM_at & V-set and transmembrane domain containiı & 1.41 & 0.00 & 0.00 \\
\hline CLC & 206207_PM_at & Charcot-Leyden crystal protein & 1.41 & 0.00 & 0.00 \\
\hline ABCA13 & 1553604_PM_at & ATP-binding cassette, sub-family $A(A B C 1)$, I & 1.41 & 0.00 & 0.00 \\
\hline OLAH & 233126_PM_s_at & oleoyl-ACP hydrolase & 1.41 & 0.00 & 0.01 \\
\hline LINC00537 & 232034_PM_at & Long intergenic non-protein coding RNA $53^{\circ}$ & 1.42 & 0.00 & 0.00 \\
\hline CD24 & 266_PM_s_at & CD24 molecule & 1.42 & 0.00 & 0.00 \\
\hline COX7B & 202110_PM_at & cytochrome c oxidase subunit VIIb & 1.42 & 0.00 & 0.01 \\
\hline OLIG2 & 213825_PM_at & oligodendrocyte lineage transcription facto & 1.42 & 0.00 & 0.01 \\
\hline DACH1 & 228915_PM_at & dachshund homolog 1 (Drosophila) & 1.43 & 0.00 & 0.00 \\
\hline INSC & 237056_PM_at & inscuteable homolog (Drosophila) & 1.43 & 0.00 & 0.00 \\
\hline--- & 230354_PM_at & --- & 1.43 & 0.00 & 0.00 \\
\hline SLC26A8 & 237340_PM_at & solute carrier family 26 , member 8 & 1.43 & 0.00 & 0.00 \\
\hline MPO & 203949_PM_at & myeloperoxidase & 1.44 & 0.00 & 0.00 \\
\hline CLEC4D & 1552772_PM_at & C-type lectin domain family 4 , member $D$ & 1.44 & 0.00 & 0.00 \\
\hline ADORA3 & 223660_PM_at & adenosine $\mathrm{A} 3$ receptor & 1.44 & 0.00 & 0.00 \\
\hline ARG1 & 231662_PM_at & Arginase, liver & 1.45 & 0.00 & 0.00 \\
\hline CEBPE & 214523_PM_at & CCAAT & 1.46 & 0.00 & 0.00 \\
\hline $\mathrm{OLAH}$ & 222945_PM_x_at & oleoyl-ACP hydrolase & 1.46 & 0.00 & 0.00 \\
\hline PRSS33 & 1552349_PM_a_at & protease, serine, 33 & 1.46 & 0.00 & 0.02 \\
\hline F5 & 231029_PM_at & coagulation factor $\mathrm{V}$ (proaccelerin, labile far & 1.47 & 0.00 & 0.00 \\
\hline PRSS33 & 1552348_PM_at & protease, serine, 33 & 1.48 & 0.00 & 0.02 \\
\hline ADORA3 & 206171_PM_at & adenosine $\mathrm{A} 3$ receptor & 1.49 & 0.00 & 0.00 \\
\hline ABCA13 & 1553605_PM_a_at & ATP-binding cassette, sub-family $A(A B C 1)$, I & 1.51 & 0.00 & 0.00 \\
\hline IL5RA & 211517_PM_s_at & interleukin 5 receptor, alpha & 1.51 & 0.00 & 0.00 \\
\hline CLEC4D & 1552773_PM_at & C-type lectin domain family 4 , member D & 1.51 & 0.00 & 0.00 \\
\hline TCN1 & 205513_PM_at & transcobalamin I (vitamin B12 binding prot $\epsilon$ & 1.52 & 0.00 & 0.00 \\
\hline MS4A3 & 1554892_PM_a_at & membrane-spanning 4-domains, subfamily & 1.52 & 0.00 & 0.00 \\
\hline RNASE2 & 206111_PM_at & ribonuclease, RNase A family, 2 (liver, eosin & 1.56 & 0.00 & 0.00 \\
\hline CAMP & 210244_PM_at & cathelicidin antimicrobial peptide & 1.58 & 0.00 & 0.00 \\
\hline HP & 208470_PM_s_at & haptoglobin & 1.59 & 0.00 & 0.00 \\
\hline SLPI & 203021_PM_at & secretory leukocyte peptidase inhibitor & 1.6 & 0.00 & 0.00 \\
\hline
\end{tabular}




\begin{tabular}{|c|c|c|c|c|c|}
\hline ANXA3 & 209369_PM_at & annexin $\mathrm{A} 3$ & 1.6 & 0.00 & 0.00 \\
\hline CD24 & 208650_PM_s_at & CD24 molecule & 1.62 & 0.00 & 0.00 \\
\hline MMP8 & 207329_PM_at & matrix metallopeptidase 8 (neutrophil colla & 1.66 & 0.00 & 0.00 \\
\hline ORM1 & 205040_PM_at & orosomucoid 1 & 1.67 & 0.00 & 0.00 \\
\hline CCL23 & 210548_PM_at & chemokine (C-C motif) ligand 23 & 1.67 & 0.00 & 0.00 \\
\hline CTSG & 205653_PM_at & cathepsin G & 1.68 & 0.00 & 0.00 \\
\hline MS4A3 & 210254_PM_at & membrane-spanning 4-domains, subfamily & 1.69 & 0.00 & 0.00 \\
\hline ORM1 & 205041_PM_s_at & orosomucoid 1 & 1.7 & 0.00 & 0.00 \\
\hline DEFA1 & 205033_PM_s_at & defensin, alpha 1 & 1.72 & 0.00 & 0.00 \\
\hline RNF182 & 230720_PM_at & ring finger protein 182 & 1.72 & 0.00 & 0.02 \\
\hline $\mathrm{HP}$ & 206697_PM_s_at & haptoglobin & 1.75 & 0.00 & 0.00 \\
\hline ARG1 & 206177_PM_s_at & arginase, liver & 1.76 & 0.00 & 0.00 \\
\hline CCL23 & 210549_PM_s_at & chemokine (C-C motif) ligand 23 & 1.86 & 0.00 & 0.00 \\
\hline CEACAM6 & 203757_PM_s_at & carcinoembryonic antigen-related cell adhe & 1.87 & 0.00 & 0.00 \\
\hline CEACAM 6 & 211657_PM_at & carcinoembryonic antigen-related cell adhe & 1.97 & 0.00 & 0.00 \\
\hline RNASE3 & 206851_PM_at & ribonuclease, RNase A family, 3 & 1.98 & 0.00 & 0.00 \\
\hline CRISP3 & 207802_PM_at & cysteine-rich secretory protein 3 & 1.98 & 0.00 & 0.00 \\
\hline BPI & 205557_PM_at & bactericidal & 2.03 & 0.00 & 0.00 \\
\hline LCN2 & 212531_PM_at & lipocalin 2 & 2.03 & 0.00 & 0.00 \\
\hline MMP8 & 231688_PM_at & matrix metallopeptidase 8 (neutrophil colla & 2.18 & 0.00 & 0.00 \\
\hline LTF & 202018_PM_s_at & lactotransferrin & 2.2 & 0.00 & 0.00 \\
\hline CEACAM 8 & 206676_PM_at & carcinoembryonic antigen-related cell adhe & 2.4 & 0.00 & 0.00 \\
\hline OLFM4 & 212768_PM_s_at & olfactomedin 4 & 2.43 & 0.00 & 0.00 \\
\hline DEFA4 & 207269_PM_at & defensin, alpha 4 , corticostatin & 2.58 & 0.00 & 0.00 \\
\hline
\end{tabular}


Supplemental Table 4 - SADS Adjusted for Cell Count

\begin{tabular}{|c|c|c|c|c|c|}
\hline symbol & probe_name & product & foldChange & pval & IFDR \\
\hline TSPAN13 & 217979_PM_at & tetraspanin 13 & -1.42 & 0.00 & 0.00 \\
\hline IGK@ & 237625_PM_s_at & immunoglobulin kappa locus & -1.41 & 0.00 & 0.00 \\
\hline--- & 233401_PM_at & --- & -1.41 & 0.00 & 0.01 \\
\hline LRRN3 & 209841_PM_s_at & leucine rich repeat neuronal 3 & -1.4 & 0.00 & 0.02 \\
\hline CLDN23 & 228707_PM_at & claudin 23 & -1.39 & 0.00 & 0.00 \\
\hline--- & 241625_PM_at & --- & -1.35 & 0.00 & 0.00 \\
\hline LIPA & 236156_PM_at & lipase A, lysosomal acid, cholesterol esterase & -1.35 & 0.00 & 0.04 \\
\hline CUX2 & 213920_PM_at & cut-like homeobox 2 & -1.34 & 0.00 & 0.00 \\
\hline EREG & 205767_PM_at & epiregulin & -1.34 & 0.00 & 0.00 \\
\hline--- & 239867_PM_at & --- & -1.33 & 0.00 & 0.01 \\
\hline EBF1 & 233261_PM_at & Early B-cell factor 1 & -1.33 & 0.00 & 0.03 \\
\hline LOC389834 & 244631_PM_at & ankyrin repeat domain 57 pseudogene & -1.32 & 0.00 & 0.02 \\
\hline YTHDC1 & 214814_PM_at & YTH domain containing 1 & -1.31 & 0.00 & 0.00 \\
\hline PRKXP1 & 235987_PM_at & protein kinase, $\mathrm{X}$-linked, pseudogene 1 & -1.31 & 0.00 & 0.02 \\
\hline LAMP3 & 205569_PM_at & lysosomal-associated membrane protein 3 & -1.31 & 0.00 & 0.05 \\
\hline GPM6B & 209167_PM_at & glycoprotein M6B & -1.3 & 0.00 & 0.00 \\
\hline LOC100506195 & 1563039_PM_at & uncharacterized LOC100506195 & -1.29 & 0.00 & 0.00 \\
\hline--- & 1561035_PM_at & --- & -1.29 & 0.00 & 0.01 \\
\hline TCF4 & 212382_PM_at & transcription factor 4 & -1.28 & 0.00 & 0.00 \\
\hline NRCAM & 204105_PM_s_at & neuronal cell adhesion molecule & -1.28 & 0.00 & 0.00 \\
\hline IL6ST & 234474_PM_x_at & interleukin 6 signal transducer (gp130, oncostatin M rec€ & -1.28 & 0.00 & 0.00 \\
\hline--- & 244181_PM_at & --- & -1.28 & 0.00 & 0.04 \\
\hline LOC389834 & 241624_PM_at & ankyrin repeat domain 57 pseudogene & -1.27 & 0.00 & 0.01 \\
\hline--- & 233425_PM_at & --- & -1.27 & 0.00 & 0.01 \\
\hline ZNF521 & 226676_PM_at & zinc finger protein 521 & -1.27 & 0.00 & 0.02 \\
\hline EBF1 & 227646_PM_at & early B-cell factor 1 & -1.27 & 0.00 & 0.04 \\
\hline MAP2K5 & 216765_PM_at & Mitogen-activated protein kinase kinase 5 & -1.26 & 0.00 & 0.00 \\
\hline ESCO1 & 235216_PM_at & establishment of cohesion 1 homolog 1 (S. cerevisiae) & -1.26 & 0.00 & 0.00 \\
\hline SAMD4A & 212845_PM_at & sterile alpha motif domain containing $4 \mathrm{~A}$ & -1.26 & 0.00 & 0.01 \\
\hline LACTB & 1552485_PM_at & lactamase, beta & -1.26 & 0.00 & 0.01 \\
\hline CCNL1 & 241495_PM_at & cyclin L1 & -1.26 & 0.00 & 0.04 \\
\hline
\end{tabular}




\begin{tabular}{|c|c|c|c|c|}
\hline SDC2 & 212158_PM_at & syndecan 2 & -1.25 & 0.00 \\
\hline IL6ST & 234967_PM_at & interleukin 6 signal transducer (gp130, oncostatin M rec€ & -1.25 & 0.00 \\
\hline TUBE1 & 230891_PM_at & Tubulin, epsilon 1 & -1.25 & 0.00 \\
\hline WSB1 & 201295_PM_s_at & WD repeat and SOCS box containing 1 & -1.25 & 0.00 \\
\hline--- & 224348_PM_s_at & --- & -1.25 & 0.00 \\
\hline--- & 230014_PM_at & --- & -1.24 & 0.00 \\
\hline HOXA3 & 235521_PM_at & homeobox A3 & -1.24 & 0.00 \\
\hline--- & 220729_PM_at & --- & -1.24 & 0.00 \\
\hline--- & 215831_PM_at & --- & -1.24 & 0.00 \\
\hline--- & 242664_PM_at & --- & -1.24 & 0.00 \\
\hline--- & 235756_PM_at & --- & -1.23 & 0.00 \\
\hline DTWD1 & 236649_PM_at & DTW domain containing 1 & -1.23 & 0.00 \\
\hline MBNL2 & 232138_PM_at & Muscleblind-like splicing regulator 2 & -1.23 & 0.00 \\
\hline RAB14 & 200928_PM_s_at & RAB14, member RAS oncogene family & -1.23 & 0.00 \\
\hline CREM & 209967_PM_s_at & cAMP responsive element modulator & -1.23 & 0.00 \\
\hline MPPED2 & 205413_PM_at & metallophosphoesterase domain containing 2 & -1.23 & 0.00 \\
\hline PTPRK & 233609_PM_at & Protein tyrosine phosphatase, receptor type, $\mathrm{K}$ & -1.23 & 0.00 \\
\hline SCLT1 & 1569190_PM_at & sodium channel and clathrin linker 1 & -1.23 & 0.00 \\
\hline--- & 238311_PM_at & --- & -1.23 & 0.00 \\
\hline--- & 1557581_PM_x_at & --- & -1.23 & 0.00 \\
\hline--- & 1557580_PM_at & --- & -1.23 & 0.00 \\
\hline--- & 1567044_PM_s_at & --- & -1.22 & 0.00 \\
\hline U2SURP & 236696_PM_at & U2 snRNP-associated SURP domain containing & -1.22 & 0.00 \\
\hline OPN3 & 224392_PM_s_at & opsin 3 & -1.22 & 0.00 \\
\hline EPT1 & 1570371_PM_a_at & ethanolaminephosphotransferase 1 (CDP-ethanolamine-! & -1.22 & 0.00 \\
\hline--- & 216072_PM_at & --- & -1.22 & 0.00 \\
\hline SMAD4 & 1565702_PM_at & SMAD family member 4 & -1.22 & 0.00 \\
\hline--- & 242713_PM_at & --- & -1.22 & 0.00 \\
\hline--- & 244331_PM_at & --- & -1.22 & 0.00 \\
\hline--- & 240789_PM_at & --- & -1.22 & 0.00 \\
\hline--- & 1559188_PM_x_at & --- & -1.22 & 0.00 \\
\hline--- & 241413_PM_at & --- & -1.21 & 0.00 \\
\hline TCF4 & 212386_PM_at & transcription factor 4 & -1.21 & 0.00 \\
\hline
\end{tabular}




\begin{tabular}{|c|c|c|c|c|c|}
\hline PPP2R3A & 209632_PM_at & protein phosphatase 2 , regulatory subunit B", alpha & -1.21 & 0.00 & 0.01 \\
\hline PTPRC & 1569830_PM_at & protein tyrosine phosphatase, receptor type, C & -1.21 & 0.00 & 0.02 \\
\hline RALGPS2 & 242458_PM_at & Ral GEF with PH domain and SH3 binding motif 2 & -1.21 & 0.00 & 0.02 \\
\hline--- & 243069_PM_at & --- & -1.21 & 0.00 & 0.02 \\
\hline MS4A7 & 224358_PM_s_at & membrane-spanning 4-domains, subfamily A, member 7 & -1.21 & 0.00 & 0.02 \\
\hline--- & 1556963_PM_at & --- & -1.2 & 0.00 & 0.00 \\
\hline--- & 235716_PM_at & --- & -1.2 & 0.00 & 0.00 \\
\hline BICD1 & 214806_PM_at & bicaudal D homolog 1 (Drosophila) & -1.2 & 0.00 & 0.00 \\
\hline--- & 236244_PM_at & --- & -1.2 & 0.00 & 0.01 \\
\hline--- & 215164_PM_at & --- & -1.2 & 0.00 & 0.01 \\
\hline--- & 239167_PM_at & --- & -1.2 & 0.00 & 0.01 \\
\hline LGALS8 & 210732_PM_s_at & lectin, galactoside-binding, soluble, 8 & -1.2 & 0.00 & 0.01 \\
\hline PID1 & 219093_PM_at & phosphotyrosine interaction domain containing 1 & -1.2 & 0.00 & 0.01 \\
\hline STYX & 242408_PM_at & serine & -1.2 & 0.00 & 0.02 \\
\hline--- & 215204_PM_at & --- & -1.2 & 0.00 & 0.02 \\
\hline--- & 242018_PM_at & --- & -1.2 & 0.00 & 0.02 \\
\hline RAB30 & 228003_PM_at & RAB30, member RAS oncogene family & -1.2 & 0.00 & 0.02 \\
\hline CCP110 & 1569353_PM_at & centriolar coiled coil protein $110 \mathrm{kDa}$ & -1.2 & 0.00 & 0.02 \\
\hline--- & 233995_PM_at & --- & -1.2 & 0.00 & 0.02 \\
\hline SMCHD1 & 241620_PM_at & structural maintenance of chromosomes flexible hinge $d ı$ & -1.2 & 0.00 & 0.02 \\
\hline SMURF2 & 227489_PM_at & SMAD specific E3 ubiquitin protein ligase 2 & -1.19 & 0.00 & 0.00 \\
\hline--- & 237821_PM_at & --- & -1.19 & 0.00 & 0.00 \\
\hline--- & 239003_PM_at & --- & -1.19 & 0.00 & 0.00 \\
\hline--- & 241320_PM_at & --- & -1.19 & 0.00 & 0.00 \\
\hline $\mathrm{COCH}$ & 1554241_PM_at & coagulation factor $\mathrm{C}$ homolog, cochlin (Limulus polypher & -1.19 & 0.00 & 0.00 \\
\hline--- & 1567045_PM_at & --- & -1.19 & 0.00 & 0.00 \\
\hline CNOT4 & 210866_PM_s_at & CCR4-NOT transcription complex, subunit 4 & -1.19 & 0.00 & 0.01 \\
\hline PCBP1-AS1 & 240017_PM_at & PCBP1 antisense RNA 1 (non-protein coding) & -1.19 & 0.00 & 0.01 \\
\hline BCL11A & 222891_PM_s_at & B-cell CLL & -1.19 & 0.00 & 0.01 \\
\hline TAF15 & 234168_PM_at & TAF15 RNA polymerase II, TATA box binding protein (TBP & -1.19 & 0.00 & 0.01 \\
\hline C16orf46 & 230281_PM_at & chromosome 16 open reading frame 46 & -1.19 & 0.00 & 0.01 \\
\hline RHPN2 & 227196_PM_at & rhophilin, Rho GTPase binding protein 2 & -1.19 & 0.00 & 0.02 \\
\hline SCARB2 & 201646_PM_at & scavenger receptor class $B$, member 2 & -1.19 & 0.00 & 0.02 \\
\hline
\end{tabular}




\begin{tabular}{|c|c|c|c|c|c|}
\hline--- & 243908_PM_at & --- & -1.19 & 0.00 & 0.02 \\
\hline KCTD6 & 238077_PM_at & potassium channel tetramerisation domain containing 6 & -1.18 & 0.00 & 0.00 \\
\hline KCMF1 & 242887_PM_at & potassium channel modulatory factor 1 & -1.18 & 0.00 & 0.00 \\
\hline --- & 236168_PM_at & --- & -1.18 & 0.00 & 0.00 \\
\hline--- & 236755_PM_at & --- & -1.18 & 0.00 & 0.00 \\
\hline $\operatorname{cox} 18$ & 227442_PM_at & COX18 cytochrome c oxidase assembly homolog (S. cerel & -1.18 & 0.00 & 0.00 \\
\hline PTPN2 & 241623_PM_at & Protein tyrosine phosphatase, non-receptor type 2 & -1.18 & 0.00 & 0.00 \\
\hline SPCS2 & 243570_PM_at & signal peptidase complex subunit 2 homolog (S. cerevisia & -1.18 & 0.00 & 0.01 \\
\hline ENAM & 223893_PM_at & enamelin & -1.18 & 0.00 & 0.01 \\
\hline CLN8 & 219341_PM_at & ceroid-lipofuscinosis, neuronal 8 (epilepsy, progressive w & -1.18 & 0.00 & 0.01 \\
\hline SCLT1 & 1569495_PM_at & sodium channel and clathrin linker 1 & -1.18 & 0.00 & 0.01 \\
\hline BCLAF1 & 201083_PM_s_at & $\mathrm{BCL} 2$-associated transcription factor 1 & -1.18 & 0.00 & 0.01 \\
\hline--- & 244625_PM_at & --- & -1.18 & 0.00 & 0.01 \\
\hline--- & 233007_PM_at & --- & -1.18 & 0.00 & 0.01 \\
\hline--- & 244341_PM_at & --- & -1.18 & 0.00 & 0.02 \\
\hline--- & 1556314_PM_a_at & --- & -1.18 & 0.00 & 0.02 \\
\hline NHLRC3 & 236953_PM_s_at & NHL repeat containing 3 & -1.17 & 0.00 & 0.00 \\
\hline ZNF785 & 1554770_PM_x_at & zinc finger protein 785 & -1.17 & 0.00 & 0.00 \\
\hline--- & 216440_PM_at & --- & -1.17 & 0.00 & 0.00 \\
\hline GART & 244822_PM_at & phosphoribosylglycinamide formyltransferase, phosphori & -1.17 & 0.00 & 0.01 \\
\hline AGPAT4 & 219693_PM_at & 1-acylglycerol-3-phosphate O-acyltransferase 4 (lysophos & -1.17 & 0.00 & 0.01 \\
\hline--- & 1557196_PM_a_at & --- & -1.17 & 0.00 & 0.01 \\
\hline CCDC50 & 225331_PM_at & coiled-coil domain containing 50 & -1.17 & 0.00 & 0.01 \\
\hline RIPK2 & 209544_PM_at & receptor-interacting serine-threonine kinase 2 & -1.17 & 0.00 & 0.01 \\
\hline PRKRA & 237107_PM_at & protein kinase, interferon-inducible double stranded RN $f$ & -1.17 & 0.00 & 0.01 \\
\hline SIKE1 & 221705_PM_s_at & suppressor of IKBKE 1 & -1.17 & 0.00 & 0.01 \\
\hline--- & 242108_PM_at & --- & -1.17 & 0.00 & 0.02 \\
\hline MSI2 & 225240_PM_s_at & musashi homolog 2 (Drosophila) & -1.16 & 0.00 & 0.00 \\
\hline RNPC3 & 226999_PM_at & RNA-binding region (RNP1, RRM) containing 3 & -1.16 & 0.00 & 0.00 \\
\hline--- & 215974_PM_at & --- & -1.16 & 0.00 & 0.01 \\
\hline--- & 232405_PM_at & --- & -1.16 & 0.00 & 0.01 \\
\hline--- & 233246_PM_at & --- & -1.16 & 0.00 & 0.02 \\
\hline ZMYM2 & 210282_PM_at & zinc finger, MYM-type 2 & -1.16 & 0.00 & 0.02 \\
\hline
\end{tabular}




\begin{tabular}{|c|c|c|c|c|c|}
\hline DIS3L2 & 238602_PM_at & DIS3 mitotic control homolog (S. cerevisiae)-like 2 & -1.15 & 0.00 & 0.00 \\
\hline ENTPD7 & 235204_PM_at & ectonucleoside triphosphate diphosphohydrolase 7 & -1.15 & 0.00 & 0.00 \\
\hline--- & 243745_PM_at & --- & -1.15 & 0.00 & 0.00 \\
\hline PCBP2 & 229467_PM_at & poly $(\mathrm{rC})$ binding protein 2 & -1.15 & 0.00 & 0.01 \\
\hline UCHL5 & 220083_PM_x_at & ubiquitin carboxyl-terminal hydrolase L5 & -1.15 & 0.00 & 0.01 \\
\hline--- & 242312_PM_x_at & --- & -1.15 & 0.00 & 0.01 \\
\hline--- & 1561640_PM_at & --- & -1.15 & 0.00 & 0.01 \\
\hline C4orf29 & 219980_PM_at & chromosome 4 open reading frame 29 & -1.15 & 0.00 & 0.01 \\
\hline TNFSF13B & 223502_PM_s_at & tumor necrosis factor (ligand) superfamily, member $13 b$ & -1.15 & 0.00 & 0.01 \\
\hline ADC & 228000_PM_at & arginine decarboxylase & -1.15 & 0.00 & 0.01 \\
\hline LOC100505658 & 1557865_PM_at & uncharacterized LOC100505658 & -1.15 & 0.00 & 0.02 \\
\hline IREB2 & 242261_PM_at & iron-responsive element binding protein 2 & -1.15 & 0.00 & 0.02 \\
\hline MED28 & 224416_PM_s_at & mediator complex subunit 28 & -1.14 & 0.00 & 0.00 \\
\hline--- & 242431_PM_at & --- & -1.14 & 0.00 & 0.01 \\
\hline--- & 240438_PM_at & --- & -1.14 & 0.00 & 0.01 \\
\hline ZNF37A & 228711_PM_at & zinc finger protein $37 \mathrm{~A}$ & -1.14 & 0.00 & 0.01 \\
\hline--- & 1556375_PM_at & --- & -1.14 & 0.00 & 0.01 \\
\hline CREM & 214508_PM_x_at & cAMP responsive element modulator & -1.14 & 0.00 & 0.01 \\
\hline NR3C1 & 232431_PM_at & nuclear receptor subfamily 3 , group $C$, member 1 (glucoc & -1.14 & 0.00 & 0.02 \\
\hline--- & 228638_PM_at & --- & -1.13 & 0.00 & 0.00 \\
\hline ZNF224 & 232427_PM_at & Zinc finger protein 224 & -1.13 & 0.00 & 0.01 \\
\hline--- & 242948_PM_x_at & --- & -1.13 & 0.00 & 0.01 \\
\hline--- & 229483_PM_at & --- & -1.13 & 0.00 & 0.01 \\
\hline LATS2 & 223380_PM_s_at & LATS, large tumor suppressor, homolog 2 (Drosophila) & -1.13 & 0.00 & 0.01 \\
\hline NAPEPLD & 238722_PM_x_at & $\mathrm{N}$-acyl phosphatidylethanolamine phospholipase $\mathrm{D}$ & -1.13 & 0.00 & 0.01 \\
\hline BTF3L4 & 235819_PM_at & Basic transcription factor 3-like 4 & -1.13 & 0.00 & 0.01 \\
\hline TDG & 203743_PM_s_at & thymine-DNA glycosylase & -1.13 & 0.00 & 0.01 \\
\hline--- & 228156_PM_at & --- & -1.13 & 0.00 & 0.01 \\
\hline ZNF785 & 1554769_PM_at & zinc finger protein 785 & -1.13 & 0.00 & 0.02 \\
\hline--- & 240121_PM_x_at & --- & -1.13 & 0.00 & 0.02 \\
\hline CDC42SE2 & 1552613_PM_s_at & CDC42 small effector 2 & -1.12 & 0.00 & 0.00 \\
\hline SLC25A14 & 204587_PM_at & solute carrier family 25 (mitochondrial carrier, brain), me & -1.12 & 0.00 & 0.01 \\
\hline ZNF747 & 238606_PM_at & zinc finger protein 747 & -1.12 & 0.00 & 0.01 \\
\hline
\end{tabular}




\begin{tabular}{|c|c|c|c|c|c|}
\hline--- & 227052_PM_at & --- & -1.12 & 0.00 & 0.01 \\
\hline AKIRIN1 & 222458_PM_s_at & akirin 1 & -1.12 & 0.00 & 0.01 \\
\hline--- & 239277_PM_at & --- & -1.12 & 0.00 & 0.01 \\
\hline VAV2 & 226063_PM_at & vav 2 guanine nucleotide exchange factor & -1.12 & 0.00 & 0.01 \\
\hline MS4A6A & 219666_PM_at & membrane-spanning 4-domains, subfamily $A$, member $6 t$ & -1.12 & 0.00 & 0.02 \\
\hline FAM131A & 221904_PM_at & family with sequence similarity 131 , member $A$ & -1.12 & 0.00 & 0.02 \\
\hline TERF1 & 203448_PM_s_at & telomeric repeat binding factor (NIMA-interacting) 1 & -1.11 & 0.00 & 0.00 \\
\hline PNISR & 212177_PM_at & PNN-interacting serine & -1.11 & 0.00 & 0.01 \\
\hline--- & 244854_PM_at & --- & -1.11 & 0.00 & 0.02 \\
\hline C1orf63 & 209006_PM_s_at & chromosome 1 open reading frame 63 & -1.11 & 0.00 & 0.02 \\
\hline GTF2H1 & 202451_PM_at & general transcription factor $\mathrm{IH}$, polypeptide $1,62 \mathrm{kDa}$ & -1.1 & 0.00 & 0.01 \\
\hline ARF4 & 201096_PM_s_at & ADP-ribosylation factor 4 & -1.1 & 0.00 & 0.01 \\
\hline ZBTB43 & 231393_PM_x_at & Zinc finger and BTB domain containing 43 & -1.1 & 0.00 & 0.01 \\
\hline MB & 204179_PM_at & myoglobin & -1.1 & 0.00 & 0.01 \\
\hline BOLA2 & 231500_PM_s_at & BolA homolog 2 (E. coli) & -1.1 & 0.00 & 0.02 \\
\hline MTMR1 & 216095_PM_x_at & myotubularin related protein 1 & -1.09 & 0.00 & 0.01 \\
\hline KIAA0907 & 202220_PM_at & KIAA0907 & -1.09 & 0.00 & 0.02 \\
\hline EIF2C2 & 225569_PM_at & Eukaryotic translation initiation factor $2 \mathrm{C}, 2$ & -1.09 & 0.00 & 0.02 \\
\hline SLC35A2 & 209326_PM_at & solute carrier family 35 (UDP-galactose transporter), mer & -1.09 & 0.00 & 0.03 \\
\hline DERL2 & 226719_PM_at & derlin 2 & -1.08 & 0.00 & 0.01 \\
\hline SLC35E1 & 227518_PM_at & solute carrier family 35 , member $\mathrm{E} 1$ & -1.08 & 0.00 & 0.02 \\
\hline FGD2 & 1553906_PM_s_at & FYVE, RhoGEF and PH domain containing 2 & -1.08 & 0.00 & 0.02 \\
\hline BBIP1 & 228993_PM_s_at & BBSome interacting protein 1 & -1.06 & 0.00 & 0.04 \\
\hline MBD4 & 214047_PM_s_at & methyl-CpG binding domain protein 4 & 1.07 & 0.00 & 0.02 \\
\hline LINC00340 & 1562469_PM_at & long intergenic non-protein coding RNA 340 & 1.08 & 0.00 & 0.01 \\
\hline BIRC6 & 224635_PM_s_at & baculoviral IAP repeat containing 6 & 1.08 & 0.00 & 0.01 \\
\hline PSMD7 & 201705_PM_at & proteasome (prosome, macropain) $26 \mathrm{~S}$ subunit, non-ATP & 1.08 & 0.00 & 0.02 \\
\hline MASP1 & 232224_PM_at & mannan-binding lectin serine peptidase 1 (C4 & 1.08 & 0.00 & 0.02 \\
\hline NAP1L4 & 201414_PM_s_s_at & nucleosome assembly protein 1-like 4 & 1.08 & 0.00 & 0.02 \\
\hline PTPRA & 213795_PM_s_at & protein tyrosine phosphatase, receptor type, A & 1.09 & 0.00 & 0.01 \\
\hline PLEKHB2 & 224121_PM_x_at & pleckstrin homology domain containing, family B (evectir & 1.09 & 0.00 & 0.02 \\
\hline USP8 & 229501_PM_s_at & ubiquitin specific peptidase 8 & 1.1 & 0.00 & 0.01 \\
\hline TTC1 & 201434_PM_-at & tetratricopeptide repeat domain 1 & 1.1 & 0.00 & 0.02 \\
\hline
\end{tabular}




$\begin{array}{cc}\text { KPNA6 } & \text { 226976_PM_at } \\ \text { MYL12A } & \text { 201319_PM_at } \\ \text { CYFIP2 } & \text { 220999_PM_S_at } \\ \text { SLC46A3 } & \text { 214719_PM_at } \\ \text { EIF1 } & \text { 217672_PM_x_at } \\ \text { GIT2 } & \text { 225558_PM_at } \\ \text { SLC35E1 } & \text { 229410_PM_at } \\ \text { TCHP } & \text { 223455_PM_at } \\ \text { DCAF5 } & \text { 224696_PM_s_at } \\ \text { ELMO2 } & \text { 221528_PM_S_at } \\ \text {-- } & \text { 1569587_PM_at } \\ \text { STXBP1 } & \text { 202260_PM_S_at } \\ \text {--- } & \text { 241617_PM_x_at } \\ \text { ELMO2 } & \text { 55692_PM_at } \\ \text { ALAS1 } & \text { 205633_PM_S_at } \\ \text { VCL } & \text { 200931_PM_S_at } \\ \text { AP2B1 } & \text { 200612_PM_S_at } \\ \text { ZNF575 } & \text { 1557119_PM_a_at } \\ \text { EEF1A1 } & \text { 1557120_PM_at } \\ \text { BHLHE40 } & \text { 201170_PM_S_at } \\ \text { LOC100509498 } & \text { 227290_PM_at } \\ \text { SOX6 } & \text { 227498_PM_at } \\ \text { TMEM189 } & \text { 201003_PM_x_at } \\ \text { SH2D3C } & \text { 226673_PM_at } \\ \text { ENDOD1 } & \text { 212573_PM_at } \\ \text { SYT11 } & \text { 209197_PM_at } \\ \text { CDC14B } & \text { 221556_PM_at } \\ \text { EHD3 } & \text { 218935_PM_at } \\ \text { F5 } & \text { 231029_PM_at } \\ \text { MGLL } & \text { 225102_PM_at } \\ \text { EIF2AK1 } & \text { 217735_PM_s_at } \\ \text { ARHGEF12 } & \text { 201334_PM_S_at } \\ \text { LINC00537 } & \text { 232034_PM_at } \\ & \end{array}$

karyopherin alpha 6 (importin alpha 7)

myosin, light chain $12 \mathrm{~A}$, regulatory, non-sarcomeric

cytoplasmic FMR1 interacting protein 2

$0.00 \quad 0.01$

solute carrier family 46, member 3

eukaryotic translation initiation factor 1

$0.00 \quad 0.01$

G protein-coupled receptor kinase interacting ArfGAP 2

$0.00 \quad 0.01$

solute carrier family 35 , member E1

$0.00 \quad 0.01$

trichoplein, keratin filament binding

$0.00 \quad 0.02$

DDB1 and CUL4 associated factor 5

$0.00 \quad 0.02$

engulfment and cell motility 2

$0.00 \quad 0.01$

---

syntaxin binding protein 1

---

engulfment and cell motility 2

aminolevulinate, delta-, synthase 1

$0.00 \quad 0.02$

$\begin{array}{lll}1.12 & 0.00 & 0.02 \\ 1.13 & 0.00 & 0.01\end{array}$

$\begin{array}{lll}1.13 & 0.00 & 0.01\end{array}$

$\begin{array}{lll}1.13 & 0.00 & 0.01\end{array}$

vinculin

adaptor-related protein complex 2 , beta 1 subunit

$\begin{array}{lll}1.14 & 0.00 & 0.01 \\ 1.14 & 0.00 & 0.01\end{array}$

$\begin{array}{lll}1.14 & 0.00 & 0.02\end{array}$

zinc finger protein 575

Eukaryotic translation elongation factor 1 alpha 1

$0.00 \quad 0.01$

basic helix-loop-helix family, member e40

$0.00 \quad 0.02$

uncharacterized LOC100509498

$0.00 \quad 0.02$

SRY (sex determining region Y)-box

$0.00 \quad 0.00$

SRY (sex determining region Y)-box 6

$0.00 \quad 0.01$

transmembrane protein 189

$0.00 \quad 0.02$

$\mathrm{SH} 2$ domain containing $3 \mathrm{C}$

endonuclease domain containing 1

$0.00 \quad 0.02$

synaptotagmin XI

$0.00 \quad 0.02$

CDC14 cell division cycle 14 homolog B (S. cerevisiae)

$0.00 \quad 0.02$

EH-domain containing 3

coagulation factor $\mathrm{V}$ (proaccelerin, labile factor)

$0.00 \quad 0.03$

monoglyceride lipase

$0.00 \quad 0.02$

eukaryotic translation initiation factor 2-alpha kinase 1

$0.00 \quad 0.05$

Rho guanine nucleotide exchange factor (GEF) 12

$0.00 \quad 0.01$

Long intergenic non-protein coding RNA 537

$0.00 \quad 0.03$

$0.00 \quad 0.03$ 


\begin{tabular}{|c|c|c|c|c|c|}
\hline ALOX12 & 207206_PM_s_at & arachidonate 12-lipoxygenase & 1.23 & 0.00 & 0.03 \\
\hline--- & 230354_PM_at & --- & 1.23 & 0.00 & 0.05 \\
\hline--- & 242094_PM_at & --- & 1.24 & 0.00 & 0.01 \\
\hline--- & 217125_PM_at & --- & 1.25 & 0.00 & 0.02 \\
\hline BEND2 & 1553842_PM_at & BEN domain containing 2 & 1.25 & 0.00 & 0.03 \\
\hline--- & 230778_PM_at & --- & 1.26 & 0.00 & 0.02 \\
\hline ACTA2 & 200974_PM_at & actin, alpha 2 , smooth muscle, aorta & 1.26 & 0.00 & 0.04 \\
\hline RIOK3 & 202131_PM_s_s_at & RIO kinase 3 (yeast) & 1.27 & 0.00 & 0.03 \\
\hline ZNF321P & 1558887_PM_at & zinc finger protein 321 , pseudogene & 1.27 & 0.00 & 0.04 \\
\hline PBX1 & 212151_PM_at & pre-B-cell leukemia homeobox 1 & 1.28 & 0.00 & 0.03 \\
\hline ARHGEF12 & 201333_PM_s_at & Rho guanine nucleotide exchange factor (GEF) 12 & 1.29 & 0.00 & 0.05 \\
\hline SLPI & 203021_PM_at & secretory leukocyte peptidase inhibitor & 1.3 & 0.00 & 0.02 \\
\hline CLU & 208792_PM_s_at & clusterin & 1.3 & 0.00 & 0.03 \\
\hline HBD & 206834_PM_at & hemoglobin, delta & 1.3 & 0.00 & 0.04 \\
\hline ITGB3 & 204627_PM_s_at & integrin, beta 3 (platelet glycoprotein IIla, antigen CD61) & 1.31 & 0.00 & 0.03 \\
\hline PPBP & 214146_PM_s_at & pro-platelet basic protein (chemokine ( $\mathrm{C}-\mathrm{X}-\mathrm{C}$ motif) liganı & 1.33 & 0.00 & 0.01 \\
\hline ITGB3 & 204628_PM_s_at & integrin, beta 3 (platelet glycoprotein IIla, antigen CD61) & 1.33 & 0.00 & 0.01 \\
\hline ITGB5 & 214021_PM_x_at & Integrin, beta 5 & 1.33 & 0.00 & 0.01 \\
\hline CLU & 208791_PM_at & clusterin & 1.33 & 0.00 & 0.01 \\
\hline CEACAM6 & 203757_PM_s_at & carcinoembryonic antigen-related cell adhesion molecul€ & 1.35 & 0.01 & 0.05 \\
\hline FAM46C & 226811_PM_at & family with sequence similarity 46 , member $C$ & 1.37 & 0.00 & 0.02 \\
\hline ORM1 & 205040_PM_at & orosomucoid 1 & 1.37 & 0.01 & 0.04 \\
\hline BPGM & 203502_PM_at & 2,3-bisphosphoglycerate mutase & 1.39 & 0.00 & 0.02 \\
\hline $\mathrm{FECH}$ & 203116_PM_s_at & ferrochelatase & 1.39 & 0.00 & 0.02 \\
\hline ORM1 & 205041_PM_s_at & orosomucoid 1 & 1.39 & 0.00 & 0.03 \\
\hline CEACAM6 & 211657_PM_at & carcinoembryonic antigen-related cell adhesion molecul€ & 1.4 & 0.00 & 0.03 \\
\hline ITGA2B & 206493_PM_at & integrin, alpha $2 \mathrm{~b}$ (platelet glycoprotein Ilb of IIb & 1.41 & 0.00 & 0.00 \\
\hline DEFA1 & 205033_PM_s_at & defensin, alpha 1 & 1.41 & 0.00 & 0.00 \\
\hline RNASE3 & 206851_PM_at & ribonuclease, RNase A family, 3 & 1.41 & 0.01 & 0.05 \\
\hline CA1 & 205950_PM_s_at & carbonic anhydrase I & 1.43 & 0.00 & 0.04 \\
\hline BPI & 205557_PM_at & bactericidal & 1.46 & 0.00 & 0.02 \\
\hline MMP8 & 231688_PM_at & matrix metallopeptidase 8 (neutrophil collagenase) & 1.47 & 0.00 & 0.04 \\
\hline LCN2 & 212531_PM_at & lipocalin 2 & 1.5 & 0.00 & 0.01 \\
\hline
\end{tabular}




\begin{tabular}{cclccc} 
CEACAM8 & 206676_PM_at & carcinoembryonic antigen-related cell adhesion molecul & 1.62 & 0.00 & 0.01 \\
LTF & 202018_PM_s_at & lactotransferrin & 1.63 & 0.00 & 0.01 \\
DEFA4 & 207269 PM_at & defensin, alpha 4, corticostatin & 1.72 & 0.00 & 0.01 \\
RNF182 & 230720_PM_at & ring finger protein 182 & 1.82 & 0.00 & 0.00 \\
HLA-DRB4 & 209728_PM_at & major histocompatibility complex, class II, DR beta 4 & 1.97 & 0.06 & 0.03 \\
\hline
\end{tabular}




\begin{tabular}{|c|c|c|c|c|c|}
\hline symbol & probe_name & product & foldChange & pval & IFDR \\
\hline LRRN3 & 209841_PM_s_at & leucine rich repeat neuronal 3 & -1.61 & 0.00 & 0.00 \\
\hline LRRN3 & 209840_PM_s_at & leucine rich repeat neuronal 3 & -1.54 & 0.00 & 0.02 \\
\hline \multirow[t]{2}{*}{ NOG } & 231798_PM_at & noggin & -1.47 & 0.00 & 0.01 \\
\hline & 233401_PM_at & & -1.47 & 0.00 & 0.01 \\
\hline IGK@ & 237625_PM_s_at & immunoglobulin kappa locus & -1.47 & 0.00 & 0.00 \\
\hline \multirow[t]{2}{*}{ IL6ST } & 234474_PM_x_at & interleukin 6 signal transducer (gp: & -1.45 & 0.00 & 0.00 \\
\hline & 224348_PM_s_at & & -1.43 & 0.00 & 0.00 \\
\hline EPPK1 & 232165_PM_at & epiplakin 1 & -1.43 & 0.00 & 0.00 \\
\hline \multirow[t]{2}{*}{ CUX2 } & 213920_PM_at & cut-like homeobox 2 & -1.42 & 0.00 & 0.00 \\
\hline & 236307_PM_at & & -1.42 & 0.00 & 0.00 \\
\hline \multirow[t]{2}{*}{ EBF1 } & 233261_PM_at & Early B-cell factor 1 & -1.42 & 0.00 & 0.00 \\
\hline & 244181_PM_at & & -1.40 & 0.00 & 0.01 \\
\hline TSPAN13 & 217979_PM_at & tetraspanin 13 & -1.40 & 0.00 & 0.00 \\
\hline EPPK1 & 232164_PM_s_at & epiplakin 1 & -1.38 & 0.00 & 0.00 \\
\hline IL6ST & 234967_PM_at & interleukin 6 signal transducer (gp: & -1.37 & 0.00 & 0.00 \\
\hline LEF1-AS1 & 243362_PM_s_at & LEF1 antisense RNA 1 (non-protein & -1.36 & 0.00 & 0.01 \\
\hline SLC16A10 & 219915_PM_s_at & solute carrier family 16, member 1 & -1.36 & 0.00 & 0.01 \\
\hline \multirow[t]{7}{*}{ COL4A3 } & 222073_PM_at & collagen, type IV, alpha 3 (Goodpa: & -1.36 & 0.00 & 0.01 \\
\hline & 228297_PM_at & & -1.35 & 0.00 & 0.01 \\
\hline & 1565913_PM_at & & -1.35 & 0.00 & 0.01 \\
\hline & 1557581_PM_x_at & & -1.34 & 0.00 & 0.00 \\
\hline & 1561035_PM_at & & -1.34 & 0.00 & 0.00 \\
\hline & 1557580_PM_at & & -1.34 & 0.00 & 0.00 \\
\hline & 243780_PM_at & & -1.34 & 0.00 & 0.00 \\
\hline LEF1-AS1 & 243363_PM_at & LEF1 antisense RNA 1 (non-protein & -1.34 & 0.00 & 0.01 \\
\hline GPM6B & 209167_PM_at & glycoprotein M6B & -1.34 & 0.00 & 0.00 \\
\hline \multirow[t]{2}{*}{ PRKXP1 } & 235987_PM_at & protein kinase, $\mathrm{X}$-linked, pseudoge & -1.34 & 0.00 & 0.02 \\
\hline & 233034_PM_at & & -1.33 & 0.00 & 0.00 \\
\hline \multirow[t]{2}{*}{ CACHD1 } & 225627_PM_s_at & cache domain containing 1 & -1.33 & 0.00 & 0.02 \\
\hline & 228858_PM_at & & -1.33 & 0.00 & 0.00 \\
\hline \multirow[t]{5}{*}{ NRCAM } & 204105_PM_s_at & neuronal cell adhesion molecule & -1.32 & 0.00 & 0.00 \\
\hline & 242664_PM_at & & -1.32 & 0.00 & 0.01 \\
\hline & 228854_PM_at & & -1.32 & 0.00 & 0.00 \\
\hline & 243107_PM_at & & -1.31 & 0.00 & 0.00 \\
\hline & 237334_PM_at & & -1.31 & 0.00 & 0.00 \\
\hline \multirow[t]{2}{*}{ LEF1 } & 210948_PM_s_at & lymphoid enhancer-binding factor & -1.31 & 0.00 & 0.00 \\
\hline & 244696_PM_at & & -1.31 & 0.00 & 0.01 \\
\hline \multirow[t]{2}{*}{ RAB11FIP3 } & 228613_PM_at & RAB11 family interacting protein 3 & -1.31 & 0.00 & 0.00 \\
\hline & 239867_PM_at & & -1.31 & 0.00 & 0.01 \\
\hline \multirow[t]{2}{*}{ MAN1C1 } & 214180_PM_at & mannosidase, alpha, class $1 \mathrm{C}$, men & -1.30 & 0.00 & 0.00 \\
\hline & 231450_PM_at & & -1.30 & 0.00 & 0.00 \\
\hline LOC100506195 & 51563039_PM_at & uncharacterized LOC100506195 & -1.30 & 0.00 & 0.00 \\
\hline \multirow[t]{4}{*}{ KDM4B } & 235789_PM_at & lysine (K)-specific demethylase 4B & -1.30 & 0.00 & 0.04 \\
\hline & 244876_PM_at & & -1.30 & 0.00 & 0.01 \\
\hline & 1554963_PM_at & & -1.30 & 0.00 & 0.00 \\
\hline & 1556932_PM_at & & -1.30 & 0.00 & 0.05 \\
\hline TNFRSF21 & 218856_PM_at & tumor necrosis factor receptor suk & -1.30 & 0.00 & 0.00 \\
\hline CLDN23 & 228707_PM_at & claudin 23 & -1.30 & 0.00 & 0.01 \\
\hline
\end{tabular}




\begin{tabular}{|c|c|c|c|c|c|}
\hline & 1556451_PM_at & & -1.29 & 0.00 & 0.00 \\
\hline TTN & 241791_PM_at & titin & -1.29 & 0.00 & 0.00 \\
\hline EBF1 & 227646_PM_at & early B-cell factor 1 & -1.29 & 0.00 & 0.02 \\
\hline & 240593_PM_x_at & & -1.29 & 0.00 & 0.00 \\
\hline & 1568366_PM_at & & -1.29 & 0.00 & 0.01 \\
\hline ZNF496 & 238145_PM_at & Zinc finger protein 496 & -1.29 & 0.00 & 0.00 \\
\hline FLJ33630 & 228686_PM_at & uncharacterized LOC644873 & -1.29 & 0.00 & 0.00 \\
\hline ZNF662 & 228538_PM_at & zinc finger protein 662 & -1.29 & 0.00 & 0.01 \\
\hline MTUS1 & 239576_PM_at & microtubule associated tumor sup & -1.29 & 0.00 & 0.00 \\
\hline & 241625_PM_at & & -1.28 & 0.00 & 0.03 \\
\hline IL6ST & 204863_PM_s_at & interleukin 6 signal transducer (gp: & -1.28 & 0.00 & 0.00 \\
\hline & 244633_PM_at & & -1.28 & 0.00 & 0.00 \\
\hline & 1556963_PM_at & & -1.28 & 0.00 & 0.00 \\
\hline & 237916_PM_at & & -1.28 & 0.00 & 0.00 \\
\hline & 1566880_PM_at & & -1.28 & 0.00 & 0.01 \\
\hline EPT1 & 1570371_PM_a_at & ethanolaminephosphotransferase & -1.28 & 0.00 & 0.00 \\
\hline & 239411_PM_at & & -1.28 & 0.00 & 0.00 \\
\hline & 232796_PM_at & & -1.27 & 0.00 & 0.00 \\
\hline AKAP2 & 202760_PM_s_at & A kinase (PRKA) anchor protein 2 & -1.27 & 0.00 & 0.01 \\
\hline & 244172_PM_at & & -1.27 & 0.00 & 0.00 \\
\hline & 241339_PM_at & & -1.27 & 0.00 & 0.03 \\
\hline PDE7A & 1552343_PM_s_at & phosphodiesterase 7A & -1.27 & 0.00 & 0.00 \\
\hline & 236796_PM_at & & -1.27 & 0.00 & 0.00 \\
\hline & 215204_PM_at & & -1.27 & 0.00 & 0.00 \\
\hline NASP & 242918_PM_at & Nuclear autoantigenic sperm prot $\epsilon$ & -1.27 & 0.00 & 0.02 \\
\hline GCOM1 & 228568_PM_at & GRINL1A complex locus 1 & -1.27 & 0.00 & 0.03 \\
\hline & 1554964_PM_x_at & & -1.27 & 0.00 & 0.00 \\
\hline PTPRK & 233609_PM_at & Protein tyrosine phosphatase, rec€ & -1.27 & 0.00 & 0.00 \\
\hline PCNP & 237577_PM_at & PEST proteolytic signal containing & -1.27 & 0.00 & 0.01 \\
\hline PPM1K & 244011_PM_at & protein phosphatase, $\mathrm{Mg} 2+$ & -1.27 & 0.00 & 0.01 \\
\hline & 237189_PM_at & & -1.27 & 0.00 & 0.03 \\
\hline & 244414_PM_at & & -1.27 & 0.00 & 0.00 \\
\hline & 241577_PM_at & & -1.27 & 0.00 & 0.01 \\
\hline & 215284_PM_at & & -1.27 & 0.00 & 0.01 \\
\hline & 242052_PM_at & & -1.26 & 0.00 & 0.00 \\
\hline TCF4 & 212382_PM_at & transcription factor 4 & -1.26 & 0.00 & 0.00 \\
\hline & 233425_PM_at & & -1.26 & 0.00 & 0.01 \\
\hline & 235756_PM_at & & -1.26 & 0.00 & 0.00 \\
\hline & 238311_PM_at & & -1.26 & 0.00 & 0.01 \\
\hline & 1556964_PM_s_at & & -1.26 & 0.00 & 0.00 \\
\hline IKZF1 & 1565818_PM_s_at & IKAROS family zinc finger 1 (Ikaros' & -1.26 & 0.00 & 0.00 \\
\hline & 236462_PM_at & & -1.26 & 0.00 & 0.01 \\
\hline & 1565915_PM_at & & -1.26 & 0.00 & 0.04 \\
\hline & 233995_PM_at & & -1.26 & 0.00 & 0.00 \\
\hline & 234044_PM_at & & -1.26 & 0.00 & 0.03 \\
\hline LOC1001S & E235167_PM_at & uncharacterized LOC100190986 & -1.26 & 0.00 & 0.00 \\
\hline & 244394_PM_at & & -1.26 & 0.00 & 0.05 \\
\hline & 1560971_PM_a_at & & -1.25 & 0.00 & 0.05 \\
\hline & 232834_PM_at & & -1.25 & 0.00 & 0.01 \\
\hline & 238157_PM_at & & -1.25 & 0.00 & 0.02 \\
\hline
\end{tabular}




\begin{tabular}{|c|c|c|c|c|c|}
\hline & 1558409_PM_at & & -1.25 & 0.00 & 0.01 \\
\hline \multirow{2}{*}{ TUBE1 } & 230891_PM_at & Tubulin, epsilon 1 & -1.25 & 0.00 & 0.01 \\
\hline & 244331_PM_at & & -1.25 & 0.00 & 0.01 \\
\hline LMLN & 238037_PM_at & leishmanolysin-like (metallopeptid & -1.25 & 0.00 & 0.00 \\
\hline \multirow[t]{4}{*}{ AGMAT } & 221648_PM_s_at & agmatine ureohydrolase (agmatina & -1.25 & 0.00 & 0.00 \\
\hline & 237953_PM_at & & -1.25 & 0.00 & 0.00 \\
\hline & 238892_PM_at & & -1.25 & 0.00 & 0.01 \\
\hline & 232795_PM_at & & -1.25 & 0.00 & 0.00 \\
\hline \multirow[t]{2}{*}{ UBE3D } & 1569262_PM_x_at & ubiquitin protein ligase E3D & -1.25 & 0.00 & 0.01 \\
\hline & 243005_PM_at & & -1.25 & 0.00 & 0.00 \\
\hline \multirow[t]{5}{*}{$\mathrm{ABCB} 4$} & 1570505_PM_at & ATP-binding cassette, sub-family B & -1.25 & 0.00 & 0.03 \\
\hline & 241320_PM_at & & -1.25 & 0.00 & 0.00 \\
\hline & 244379_PM_at & & -1.25 & 0.00 & 0.01 \\
\hline & 236315_PM_at & & -1.25 & 0.00 & 0.01 \\
\hline & 220854_PM_at & & -1.25 & 0.00 & 0.01 \\
\hline ZFP112 & 236328_PM_at & zinc finger protein 112 homolog ( $\mathrm{n}$ & -1.25 & 0.00 & 0.01 \\
\hline SLFNL1 & 1553315_PM_at & schlafen-like 1 & -1.25 & 0.00 & 0.00 \\
\hline \multirow[t]{2}{*}{ NUP62 } & 236505_PM_at & Nucleoporin 62kDa & -1.25 & 0.00 & 0.00 \\
\hline & 232614_PM_at & & -1.25 & 0.00 & 0.03 \\
\hline YTHDC1 & 214814_PM_at & YTH domain containing 1 & -1.24 & 0.00 & 0.01 \\
\hline CAMTA1 & 1555370_PM_a_at & calmodulin binding transcription a & -1.24 & 0.00 & 0.02 \\
\hline \multirow[t]{3}{*}{ AAK1 } & 205434_PM_s_at & AP2 associated kinase 1 & -1.24 & 0.00 & 0.00 \\
\hline & 241893_PM_at & & -1.24 & 0.00 & 0.00 \\
\hline & 239788_PM_at & & -1.24 & 0.00 & 0.01 \\
\hline CLN8 & 219341_PM_at & ceroid-lipofuscinosis, neuronal 8 ( $\epsilon$ & -1.24 & 0.00 & 0.00 \\
\hline MS4A1 & 231418_PM_at & Membrane-spanning 4-domains, s। & -1.24 & 0.00 & 0.02 \\
\hline \multirow[t]{2}{*}{ SERPINB9 } & 242814_PM_at & serpin peptidase inhibitor, clade B & -1.24 & 0.00 & 0.01 \\
\hline & 234822_PM_at & & -1.24 & 0.00 & 0.02 \\
\hline \multirow[t]{2}{*}{ APBA2 } & 209870_PM_s_at & amyloid beta (A4) precursor protei & -1.24 & 0.00 & 0.00 \\
\hline & 243450_PM_at & & -1.24 & 0.00 & 0.00 \\
\hline \multirow[t]{2}{*}{ POU6F1 } & 229809_PM_at & POU class 6 homeobox 1 & -1.24 & 0.00 & 0.01 \\
\hline & 239003_PM_at & & -1.24 & 0.00 & 0.00 \\
\hline \multirow[t]{2}{*}{ AKT3 } & 222880_PM_at & v-akt murine thymoma viral oncog & -1.24 & 0.00 & 0.00 \\
\hline & 1560259_PM_at & & -1.24 & 0.00 & 0.01 \\
\hline U2SURP & 236696_PM_at & U2 snRNP-associated SURP domair & -1.24 & 0.00 & 0.00 \\
\hline PAX5 & 221969_PM_at & paired box 5 & -1.24 & 0.00 & 0.02 \\
\hline \multirow[t]{5}{*}{ METTL6 } & 232102_PM_at & Methyltransferase like 6 & -1.24 & 0.00 & 0.00 \\
\hline & 1559188_PM_x_at & & -1.24 & 0.00 & 0.01 \\
\hline & 1563217_PM_at & & -1.24 & 0.00 & 0.02 \\
\hline & 236617_PM_at & & -1.24 & 0.00 & 0.01 \\
\hline & 237432_PM_at & & -1.24 & 0.00 & 0.00 \\
\hline \multirow[t]{2}{*}{ SRGAP2 } & 1556202_PM_at & SLIT-ROBO Rho GTPase activating | & -1.24 & 0.00 & 0.04 \\
\hline & 216211_PM_at & & -1.24 & 0.00 & 0.01 \\
\hline ATAD5 & 220223_PM_at & ATPase family, AAA domain contai & -1.24 & 0.00 & 0.00 \\
\hline \multirow[t]{3}{*}{ ACAT1 } & 1559239_PM_s_at & acetyl-CoA acetyltransferase 1 & -1.24 & 0.00 & 0.01 \\
\hline & 217536_PM_x_at & & -1.24 & 0.00 & 0.05 \\
\hline & 1561004_PM_at & & -1.24 & 0.00 & 0.04 \\
\hline STRBP & 233252_PM_s_at & spermatid perinuclear RNA bindin€ & -1.24 & 0.00 & 0.01 \\
\hline \multirow[t]{2}{*}{ SRRM2 } & 1554671_PM_a_at & serine & -1.23 & 0.00 & 0.02 \\
\hline & 241505_PM_at & & -1.23 & 0.00 & 0.03 \\
\hline
\end{tabular}




\begin{tabular}{|c|c|c|c|c|c|}
\hline & 1558410_PM_s_at & & -1.23 & 0.00 & 0.00 \\
\hline & 241007_PM_at & & -1.23 & 0.00 & 0.00 \\
\hline & 240789_PM_at & & -1.23 & 0.00 & 0.01 \\
\hline & 1556314_PM_a_at & & -1.23 & 0.00 & 0.00 \\
\hline LOC389834 & 241624_PM_at & ankyrin repeat domain 57 pseudo६ & -1.23 & 0.00 & 0.04 \\
\hline RCAN3 & 219864_PM_s_at & RCAN family member 3 & -1.23 & 0.00 & 0.05 \\
\hline & 240971_PM_x_at & & -1.23 & 0.00 & 0.01 \\
\hline LILRA4 & 210313_PM_at & leukocyte immunoglobulin-like rec & -1.23 & 0.00 & 0.01 \\
\hline & 222266_PM_at & & -1.23 & 0.00 & 0.00 \\
\hline $\mathrm{ZCCHC2}$ & 219062_PM_s_at & zinc finger, $\mathrm{CCHC}$ domain containir & -1.23 & 0.00 & 0.01 \\
\hline & 1567044_PM_s_at & & -1.23 & 0.00 & 0.00 \\
\hline CAND2 & 213547_PM_at & cullin-associated and neddylation-ı & -1.23 & 0.00 & 0.02 \\
\hline & 242886_PM_at & & -1.23 & 0.00 & 0.01 \\
\hline & 230014_PM_at & & -1.23 & 0.00 & 0.03 \\
\hline & 216072_PM_at & & -1.23 & 0.00 & 0.01 \\
\hline BLNK & 207655_PM_s_at & B-cell linker & -1.23 & 0.00 & 0.01 \\
\hline RALGAPA1 & 234923_PM_at & Ral GTPase activating protein, alph & -1.23 & 0.00 & 0.01 \\
\hline LARS & 223888_PM_s_at & leucyl-tRNA synthetase & -1.23 & 0.00 & 0.00 \\
\hline MAP2K5 & 216765_PM_at & Mitogen-activated protein kinase & -1.23 & 0.00 & 0.01 \\
\hline ZNF275 & 225383_PM_at & zinc finger protein 275 & -1.23 & 0.00 & 0.00 \\
\hline & 239227_PM_at & & -1.23 & 0.00 & 0.03 \\
\hline CEP41 & 225484_PM_at & centrosomal protein $41 \mathrm{kDa}$ & -1.23 & 0.00 & 0.00 \\
\hline DHX30 & 230871_PM_at & DEAH (Asp-Glu-Ala-His) box polyp€ & -1.23 & 0.00 & 0.00 \\
\hline CCR6 & 206983_PM_at & chemokine (C-C motif) receptor 6 & -1.23 & 0.00 & 0.00 \\
\hline & 237429_PM_at & & -1.23 & 0.00 & 0.01 \\
\hline & 1567045_PM_at & & -1.22 & 0.00 & 0.00 \\
\hline SCML1 & 222747_PM_s_at & sex comb on midleg-like 1 (Drosop & -1.22 & 0.00 & 0.01 \\
\hline NAF1 & 244828_PM_x_at & nuclear assembly factor 1 homolo६ & -1.22 & 0.00 & 0.00 \\
\hline KIAA0368 & 236368_PM_at & KIAA0368 & -1.22 & 0.00 & 0.00 \\
\hline BCL11A & 1559078_PM_at & B-cell CLL & -1.22 & 0.00 & 0.00 \\
\hline & 239842_PM_x_at & & -1.22 & 0.00 & 0.01 \\
\hline LOC100507539 & c 244308_PM_at & uncharacterized LOC100507539 & -1.22 & 0.00 & 0.00 \\
\hline & 243546_PM_at & & -1.22 & 0.00 & 0.02 \\
\hline APBA2 & 209871_PM_s_at & amyloid beta (A4) precursor protei & -1.22 & 0.00 & 0.02 \\
\hline MUM1 & 221290_PM_s_at & melanoma associated antigen (mu & -1.22 & 0.00 & 0.00 \\
\hline SMCHD1 & 241620_PM_at & structural maintenance of chromo: & -1.22 & 0.00 & 0.01 \\
\hline MBNL2 & 232138_PM_at & Muscleblind-like splicing regulator & -1.22 & 0.00 & 0.00 \\
\hline & 1560861_PM_at & & -1.22 & 0.00 & 0.03 \\
\hline LOC100131541 & 11558569_PM_at & Uncharacterized LOC100131541 & -1.22 & 0.00 & 0.01 \\
\hline & 222098_PM_s_at & & -1.22 & 0.00 & 0.00 \\
\hline C16orf46 & 230281_PM_at & chromosome 16 open reading fran & -1.22 & 0.00 & 0.00 \\
\hline $\mathrm{BACH} 2$ & 227173_PM_s_at & BTB and CNC homology 1 , basic leı & -1.22 & 0.00 & 0.00 \\
\hline TMEM67 & 1569377_PM_at & transmembrane protein 67 & -1.22 & 0.00 & 0.03 \\
\hline & 236279_PM_at & & -1.22 & 0.00 & 0.00 \\
\hline SLFN5 & 1557078_PM_at & schlafen family member 5 & -1.22 & 0.00 & 0.04 \\
\hline FAM171A1 & 212771_PM_at & family with sequence similarity 17 : & -1.22 & 0.00 & 0.00 \\
\hline SLC16A10 & 222939_PM_s_at & solute carrier family 16, member 1 & -1.22 & 0.00 & 0.02 \\
\hline & 239397_PM_at & & -1.22 & 0.00 & 0.00 \\
\hline & 244423_PM_at & & -1.22 & 0.00 & 0.03 \\
\hline RAB30 & 228003_PM_at & RAB30, member RAS oncogene far & -1.22 & 0.00 & 0.00 \\
\hline
\end{tabular}




\begin{tabular}{|c|c|c|c|c|c|}
\hline ZNF785 & 1554770_PM_x_at & zinc finger protein 785 & -1.22 & 0.00 & 0.00 \\
\hline \multirow[t]{4}{*}{ FAM226B } & 242292_PM_at & family with sequence similarity 221 & -1.22 & 0.00 & 0.01 \\
\hline & 243473_PM_at & & -1.22 & 0.00 & 0.02 \\
\hline & 233246_PM_at & & -1.22 & 0.00 & 0.00 \\
\hline & 244665_PM_at & & -1.22 & 0.00 & 0.02 \\
\hline \multirow[t]{3}{*}{ CCDC50 } & 228693_PM_at & coiled-coil domain containing 50 & -1.22 & 0.00 & 0.02 \\
\hline & 238536_PM_at & & -1.22 & 0.00 & 0.01 \\
\hline & 240622_PM_at & & -1.22 & 0.00 & 0.00 \\
\hline \multirow[t]{2}{*}{ AUTS2 } & 212599_PM_at & autism susceptibility candidate 2 & -1.22 & 0.00 & 0.02 \\
\hline & 239167_PM_at & & -1.22 & 0.00 & 0.00 \\
\hline \multirow[t]{2}{*}{ LOC100291323 } & 31564639_PM_at & uncharacterized LOC100291323 & -1.22 & 0.00 & 0.00 \\
\hline & 1556352_PM_at & & -1.22 & 0.00 & 0.02 \\
\hline AQP3 & 39249_PM_at & aquaporin 3 (Gill blood group) & -1.22 & 0.00 & 0.00 \\
\hline \multirow[t]{3}{*}{ PSMG4 } & 242055_PM_at & Proteasome (prosome, macropain' & -1.22 & 0.00 & 0.01 \\
\hline & 235912_PM_at & & -1.22 & 0.00 & 0.00 \\
\hline & 240216_PM_at & & -1.21 & 0.00 & 0.01 \\
\hline PLEKHA1 & 219024_PM_at & pleckstrin homology domain conta & -1.21 & 0.00 & 0.01 \\
\hline \multirow{3}{*}{ FAM213A } & 228155_PM_at & family with sequence similarity $21:$ & -1.21 & 0.00 & 0.00 \\
\hline & 242270_PM_at & & -1.21 & 0.00 & 0.01 \\
\hline & 230702_PM_at & & -1.21 & 0.00 & 0.01 \\
\hline SPIB & 232739_PM_at & Spi-B transcription factor (Spi-1 & -1.21 & 0.00 & 0.02 \\
\hline TMEM97 & 212282_PM_at & transmembrane protein 97 & -1.21 & 0.00 & 0.00 \\
\hline C14orf64 & 1559097_PM_at & chromosome 14 open reading fran & -1.21 & 0.00 & 0.00 \\
\hline \multirow{3}{*}{ NMNAT3 } & 228090_PM_at & nicotinamide nucleotide adenylyltı & -1.21 & 0.00 & 0.01 \\
\hline & 242104_PM_at & & -1.21 & 0.00 & 0.02 \\
\hline & 242920_PM_at & & -1.21 & 0.00 & 0.00 \\
\hline \multirow[t]{2}{*}{ ZBTB20 } & 205383_PM_s_at & zinc finger and BTB domain contair & -1.21 & 0.00 & 0.00 \\
\hline & 1565614_PM_at & & -1.21 & 0.00 & 0.01 \\
\hline AXIN2 & 222696_PM_at & $\operatorname{axin} 2$ & -1.21 & 0.00 & 0.01 \\
\hline \multirow[t]{5}{*}{$\mathrm{ZCCHC2}$} & 224503_PM_s_at & zinc finger, $\mathrm{CCHC}$ domain containir & -1.21 & 0.00 & 0.02 \\
\hline & 1556769_PM_a_at & & -1.21 & 0.00 & 0.02 \\
\hline & 233690_PM_at & & -1.21 & 0.00 & 0.01 \\
\hline & 243030_PM_at & & -1.21 & 0.00 & 0.00 \\
\hline & 229465_PM_s_at & & -1.21 & 0.00 & 0.01 \\
\hline \multirow[t]{2}{*}{ ZMYM6 } & 1552969_PM_a_at & zinc finger, MYM-type 6 & -1.21 & 0.00 & 0.01 \\
\hline & 1569597_PM_at & & -1.21 & 0.00 & 0.00 \\
\hline SHMT2 & 214095_PM_at & serine hydroxymethyltransferase 2 & -1.21 & 0.00 & 0.00 \\
\hline GAL3ST4 & 219815_PM_at & galactose-3-O-sulfotransferase 4 & -1.21 & 0.00 & 0.01 \\
\hline \multirow[t]{4}{*}{ BICD1 } & 214806_PM_at & bicaudal D homolog 1 (Drosophila) & -1.21 & 0.00 & 0.00 \\
\hline & 244878_PM_at & & -1.21 & 0.00 & 0.01 \\
\hline & 231107_PM_at & & -1.21 & 0.00 & 0.03 \\
\hline & 232778_PM_at & & -1.21 & 0.00 & 0.01 \\
\hline \multirow[t]{2}{*}{ TNPO1 } & 1556116_PM_s_at & Transportin 1 & -1.21 & 0.00 & 0.00 \\
\hline & 239646_PM_at & & -1.21 & 0.00 & 0.00 \\
\hline TAF15 & 234168_PM_at & TAF15 RNA polymerase II, TATA bc & -1.21 & 0.00 & 0.00 \\
\hline AQP3 & 203747_PM_at & aquaporin 3 (Gill blood group) & -1.21 & 0.00 & 0.00 \\
\hline ENAM & 223893_PM_at & enamelin & -1.21 & 0.00 & 0.00 \\
\hline \multirow[t]{2}{*}{ NSUN6 } & 230200_PM_at & NOP2 & -1.21 & 0.00 & 0.00 \\
\hline & 240605_PM_at & & -1.21 & 0.00 & 0.00 \\
\hline SOX7 & 228698_PM_at & SRY (sex determining region Y)-bo) & -1.21 & 0.00 & 0.02 \\
\hline
\end{tabular}




\begin{tabular}{|c|c|c|c|c|c|}
\hline CBX5 & 226085_PM_at & chromobox homolog 5 & -1.21 & 0.00 & 0.00 \\
\hline CHD1L & 238070_PM_at & chromodomain helicase DNA bindi & -1.21 & 0.00 & 0.00 \\
\hline \multirow[t]{5}{*}{ NAA25 } & 236816_PM_at & $\mathrm{N}($ alpha)-acetyltransferase $25, \mathrm{Nat}$ & -1.21 & 0.00 & 0.00 \\
\hline & 1564776_PM_at & & -1.21 & 0.00 & 0.01 \\
\hline & 238610_PM_s_at & & -1.21 & 0.00 & 0.01 \\
\hline & 244625_PM_at & & -1.20 & 0.00 & 0.00 \\
\hline & 1569601_PM_at & & -1.20 & 0.00 & 0.01 \\
\hline AURKAPS1 & 216057_PM_at & aurora kinase $\mathrm{A}$ pseudogene 1 & -1.20 & 0.00 & 0.00 \\
\hline LMO7 & 242722_PM_at & LIM domain 7 & -1.20 & 0.00 & 0.01 \\
\hline CYP2B6 & 206754_PM_s_at & cytochrome P450, family 2 , subfan & -1.20 & 0.00 & 0.02 \\
\hline MAP2K6 & 205699_PM_at & mitogen-activated protein kinase $\mathrm{k}$ & -1.20 & 0.00 & 0.01 \\
\hline \multirow[t]{2}{*}{ AKAP13 } & 229783_PM_at & A kinase (PRKA) anchor protein 13 & -1.20 & 0.00 & 0.01 \\
\hline & 243736_PM_at & & -1.20 & 0.00 & 0.00 \\
\hline METAP1D & 227805_PM_at & methionyl aminopeptidase type 1[ & -1.20 & 0.00 & 0.00 \\
\hline \multirow[t]{2}{*}{ MPRIP } & 214694_PM_at & myosin phosphatase Rho interactiı & -1.20 & 0.00 & 0.00 \\
\hline & 240595_PM_at & & -1.20 & 0.00 & 0.00 \\
\hline RIMKLB & 226164_PM_x_at & ribosomal modification protein rin & -1.20 & 0.00 & 0.00 \\
\hline LRPPRC & 1557360_PM_at & leucine-rich pentatricopeptide rep & -1.20 & 0.00 & 0.00 \\
\hline \multirow[t]{3}{*}{ CD27 } & 206150_PM_at & CD27 molecule & -1.20 & 0.00 & 0.01 \\
\hline & 1561195_PM_at & & -1.20 & 0.00 & 0.01 \\
\hline & 215164_PM_at & & -1.20 & 0.00 & 0.01 \\
\hline CARD11 & 1562368_PM_at & caspase recruitment domain famil & -1.20 & 0.00 & 0.00 \\
\hline ZNF141 & 235580_PM_at & zinc finger protein 141 & -1.20 & 0.00 & 0.00 \\
\hline \multirow[t]{3}{*}{ LOC10050705 } & 31559240_PM_at at & uncharacterized LOC100507053 & -1.20 & 0.00 & 0.01 \\
\hline & 236379_PM_at & & -1.20 & 0.00 & 0.00 \\
\hline & 241106_PM_at & & -1.20 & 0.00 & 0.00 \\
\hline SNX19 & 1554986_PM_a_at & sorting nexin 19 & -1.20 & 0.00 & 0.02 \\
\hline \multirow[t]{3}{*}{ ZNF587 } & 1557248_PM_at & Zinc finger protein 587 & -1.20 & 0.00 & 0.01 \\
\hline & 1556375_PM_at & & -1.20 & 0.00 & 0.00 \\
\hline & 240438_PM_at & & -1.20 & 0.00 & 0.00 \\
\hline \multirow[t]{2}{*}{ RHPN2 } & 227196_PM_at & rhophilin, Rho GTPase binding prot & -1.20 & 0.00 & 0.01 \\
\hline & 236143_PM_at & & -1.20 & 0.00 & 0.01 \\
\hline TMEM63A & 215583_PM_at & Transmembrane protein 63A & -1.20 & 0.00 & 0.00 \\
\hline \multirow[t]{3}{*}{ IL28RA } & 244261_PM_at & interleukin 28 receptor, alpha (int $\epsilon$ & -1.20 & 0.00 & 0.01 \\
\hline & 244845_PM_at & & -1.20 & 0.00 & 0.02 \\
\hline & 218544_PM_s_at & & -1.20 & 0.00 & 0.00 \\
\hline RIPK4 & 221215_PM_s_at & receptor-interacting serine-threon & -1.20 & 0.00 & 0.02 \\
\hline SLC38A10 & 1563226_PM_at & solute carrier family 38, member 1 & -1.20 & 0.00 & 0.00 \\
\hline SRRM2 & 208610_PM_s_at & serine & -1.20 & 0.00 & 0.01 \\
\hline FLJ10038 & 205511_PM_at & uncharacterized protein FL10038 & -1.20 & 0.00 & 0.01 \\
\hline \multirow[t]{2}{*}{ ETS1 } & 1555355_PM_a_at & v-ets erythroblastosis virus E26 on & -1.20 & 0.00 & 0.00 \\
\hline & 242947_PM_at & & -1.20 & 0.00 & 0.02 \\
\hline URB1 & 212996_PM_s_at & URB1 ribosome biogenesis 1 homc & -1.20 & 0.00 & 0.00 \\
\hline BCL11A & 222891_PM_s_at & B-cell CLL & -1.20 & 0.00 & 0.00 \\
\hline \multirow[t]{2}{*}{ C14orf64 } & 1564211_PM_at & chromosome 14 open reading fran & -1.20 & 0.00 & 0.00 \\
\hline & 239449_PM_at & & -1.20 & 0.00 & 0.01 \\
\hline \multirow[t]{2}{*}{ MAN1C1 } & 218918_PM_at & mannosidase, alpha, class $1 \mathrm{C}$, men & -1.20 & 0.00 & 0.00 \\
\hline & 231351_PM_at & & -1.20 & 0.00 & 0.01 \\
\hline TCF4 & 212386_PM_at & transcription factor 4 & -1.20 & 0.00 & 0.01 \\
\hline TGIF2 & 218724_PM_s_at & TGFB-induced factor homeobox 2 & -1.20 & 0.00 & 0.00 \\
\hline
\end{tabular}




\begin{tabular}{|c|c|c|c|c|c|}
\hline & 243593_PM_s_at & & -1.20 & 0.00 & 0.02 \\
\hline \multirow[t]{3}{*}{ CAMSAP1 } & 244682_PM_at & calmodulin regulated spectrin-assc & -1.19 & 0.00 & 0.01 \\
\hline & 242709_PM_s_at & & -1.19 & 0.00 & 0.01 \\
\hline & 237998_PM_at & & -1.19 & 0.00 & 0.01 \\
\hline \multirow[t]{3}{*}{ SPATS2 } & 222593_PM_s_at & spermatogenesis associated, serin & -1.19 & 0.00 & 0.00 \\
\hline & 244341_PM_at & & -1.19 & 0.00 & 0.01 \\
\hline & 243817_PM_at & & -1.19 & 0.00 & 0.02 \\
\hline \multirow[t]{3}{*}{ TARSL2 } & 227611_PM_at & threonyl-tRNA synthetase-like 2 & -1.19 & 0.00 & 0.00 \\
\hline & 240655_PM_at & & -1.19 & 0.00 & 0.00 \\
\hline & 231222_PM_at & & -1.19 & 0.00 & 0.01 \\
\hline TSPAN3 & 200972_PM_at & tetraspanin 3 & -1.19 & 0.00 & 0.01 \\
\hline \multirow[t]{2}{*}{ TAF4B } & 235020_PM_at & TAF4b RNA polymerase II, TATA bc & -1.19 & 0.00 & 0.01 \\
\hline & 242031_PM_at & & -1.19 & 0.00 & 0.01 \\
\hline PTPLAD1 & 234000_PM_s_at & protein tyrosine phosphatase-like & -1.19 & 0.00 & 0.01 \\
\hline BCL7A & 203796_PM_s_at & B-cell CLL & -1.19 & 0.00 & 0.00 \\
\hline COPG2IT1 & 213486_PM_at & COPG2 imprinted transcript 1 (non & -1.19 & 0.00 & 0.00 \\
\hline TGFBR1 & 236561_PM_at & transforming growth factor, beta $r$ & -1.19 & 0.00 & 0.00 \\
\hline PCBP2 & 229467_PM_at & poly $(\mathrm{rC})$ binding protein 2 & -1.19 & 0.00 & 0.00 \\
\hline ATXN3 & 205415_PM_s_at & ataxin 3 & -1.19 & 0.00 & 0.00 \\
\hline SLC11A2 & 237106_PM_at & Solute carrier family 11 (proton-co & -1.19 & 0.00 & 0.01 \\
\hline \multirow[t]{2}{*}{ THRAP3 } & 242163_PM_at & thyroid hormone receptor associat & -1.19 & 0.00 & 0.01 \\
\hline & 239238_PM_at & & -1.19 & 0.00 & 0.00 \\
\hline CXCR7 & 212977_PM_at & chemokine (C-X-C motif) receptor & -1.19 & 0.00 & 0.01 \\
\hline \multirow[t]{3}{*}{ ZNF785 } & 1554769_PM_at & zinc finger protein 785 & -1.19 & 0.00 & 0.00 \\
\hline & 229858_PM_at & & -1.19 & 0.00 & 0.02 \\
\hline & 1562412_PM_at & & -1.19 & 0.00 & 0.01 \\
\hline OPN3 & 224392_PM_s_at & opsin 3 & -1.19 & 0.00 & 0.01 \\
\hline SLC38A10 & 1563228_PM_x_at & solute carrier family 38, member 1 & -1.19 & 0.00 & 0.01 \\
\hline \multirow[t]{2}{*}{ TXLNG } & 219969_PM_at & taxilin gamma & -1.19 & 0.00 & 0.00 \\
\hline & 1561640_PM_at & & -1.19 & 0.00 & 0.00 \\
\hline DTWD1 & 236649_PM_at & DTW domain containing 1 & -1.19 & 0.00 & 0.00 \\
\hline \multirow[t]{5}{*}{ SLC16A7 } & 210807_PM_s_at & solute carrier family 16, member 7 & -1.19 & 0.00 & 0.01 \\
\hline & 243286_PM_at & & -1.19 & 0.00 & 0.00 \\
\hline & 237001_PM_at & & -1.19 & 0.00 & 0.01 \\
\hline & 233376_PM_at & & -1.18 & 0.00 & 0.01 \\
\hline & 1558686_PM_at & & -1.18 & 0.00 & 0.01 \\
\hline DPP4 & 203716_PM_s_at & dipeptidyl-peptidase 4 & -1.18 & 0.00 & 0.01 \\
\hline \multirow[t]{4}{*}{ PHF20 } & 1569906_PM_s_sat & PHD finger protein 20 & -1.18 & 0.00 & 0.00 \\
\hline & 1560680_PM_at & & -1.18 & 0.00 & 0.00 \\
\hline & 241413_PM_at & & -1.18 & 0.00 & 0.01 \\
\hline & 240007_PM_at & & -1.18 & 0.00 & 0.01 \\
\hline TCF4 & 212387_PM_at & transcription factor 4 & -1.18 & 0.00 & 0.00 \\
\hline PEX3 & 203969_PM_at & peroxisomal biogenesis factor 3 & -1.18 & 0.00 & 0.00 \\
\hline \multirow{2}{*}{ ZFAND6 } & 239757_PM_at & Zinc finger, AN1-type domain 6 & -1.18 & 0.00 & 0.00 \\
\hline & 233243_PM_at & & -1.18 & 0.00 & 0.00 \\
\hline \multirow[t]{4}{*}{ ZDHHC21 } & 229923_PM_at & zinc finger, DHHC-type containing & -1.18 & 0.00 & 0.01 \\
\hline & 243675_PM_at & & -1.18 & 0.00 & 0.00 \\
\hline & 234649_PM_at & & -1.18 & 0.00 & 0.00 \\
\hline & 236237_PM_at & & -1.18 & 0.00 & 0.00 \\
\hline ZNF202 & 204327_PM_s_at & zinc finger protein 202 & -1.18 & 0.00 & 0.00 \\
\hline
\end{tabular}




\begin{tabular}{|c|c|c|c|c|c|}
\hline$A D C$ & 228000_PM_at & arginine decarboxylase & -1.18 & 0.00 & 0.00 \\
\hline GKAP1 & 229312_PM_s_at & G kinase anchoring protein 1 & -1.18 & 0.00 & 0.00 \\
\hline \multirow[t]{3}{*}{ RBM5 } & 230742_PM_at & RNA binding motif protein 5 & -1.18 & 0.00 & 0.00 \\
\hline & 242298_PM_x_at & & -1.18 & 0.00 & 0.01 \\
\hline & 238271_PM_x_at & & -1.18 & 0.00 & 0.01 \\
\hline PRKRA & 237107_PM_at & protein kinase, interferon-inducibl & -1.18 & 0.00 & 0.00 \\
\hline PIK3IP1 & 221757_PM_at & phosphoinositide-3-kinase interact & -1.18 & 0.00 & 0.00 \\
\hline \multirow[t]{2}{*}{ BCL11A } & 210347_PM_s_at & B-cell CLL & -1.18 & 0.00 & 0.00 \\
\hline & 237067_PM_at & & -1.18 & 0.00 & 0.01 \\
\hline ABLIM1 & 210461_PM_s_at & actin binding LIM protein 1 & -1.18 & 0.00 & 0.01 \\
\hline \multirow[t]{2}{*}{ GART } & 244822_PM_at & phosphoribosylglycinamide formyl & -1.18 & 0.00 & 0.01 \\
\hline & 244145_PM_at & & -1.18 & 0.00 & 0.01 \\
\hline \multirow[t]{3}{*}{ ZMYM2 } & 210282_PM_at & zinc finger, MYM-type 2 & -1.18 & 0.00 & 0.01 \\
\hline & 236066_PM_at & & -1.18 & 0.00 & 0.00 \\
\hline & 238040_PM_at & & -1.18 & 0.00 & 0.01 \\
\hline \multirow[t]{2}{*}{ SPG7 } & 230885_PM_at & spastic paraplegia 7 (pure and corr & -1.18 & 0.00 & 0.00 \\
\hline & 239110_PM_s_at & & -1.18 & 0.00 & 0.01 \\
\hline TOX4 & 232097_PM_at & TOX high mobility group box famil & -1.18 & 0.00 & 0.01 \\
\hline \multirow[t]{2}{*}{ MORC4 } & 219038_PM_at & MORC family $C W$-type zinc finger $\angle$ & -1.18 & 0.00 & 0.00 \\
\hline & 236210_PM_at & & -1.17 & 0.00 & 0.01 \\
\hline TMEM97 & 212281_PM_s_at & transmembrane protein 97 & -1.17 & 0.00 & 0.01 \\
\hline \multirow[t]{2}{*}{ DIS3L2 } & 238602_PM_at & DIS3 mitotic control homolog (S. Ct & -1.17 & 0.00 & 0.00 \\
\hline & 244204_PM_at & & -1.17 & 0.00 & 0.01 \\
\hline IREB2 & 242261_PM_at & iron-responsive element binding $p$ & -1.17 & 0.00 & 0.00 \\
\hline PSPC1 & 222611_PM_s_at & paraspeckle component 1 & -1.17 & 0.00 & 0.00 \\
\hline MAP4K4 & 1558732_PM_at & mitogen-activated protein kinase $\mathrm{k}$ & -1.17 & 0.00 & 0.01 \\
\hline NXPE3 & 243011_PM_at & neurexophilin and PC-esterase dor & -1.17 & 0.00 & 0.00 \\
\hline \multirow[t]{2}{*}{ ZNF37A } & 228711_PM_at & zinc finger protein 37A & -1.17 & 0.00 & 0.00 \\
\hline & 243262_PM_at & & -1.17 & 0.00 & 0.00 \\
\hline \multirow[t]{3}{*}{ UGP2 } & 232180_PM_at & UDP-glucose pyrophosphorylase 2 & -1.17 & 0.00 & 0.00 \\
\hline & 1563109_PM_at & & -1.17 & 0.00 & 0.01 \\
\hline & 239943_PM_x_at & & -1.17 & 0.00 & 0.01 \\
\hline SFXN4 & 230637_PM_at & sideroflexin 4 & -1.17 & 0.00 & 0.00 \\
\hline CWF19L2 & 237040_PM_at & CWF19-like 2, cell cycle control (S. & -1.17 & 0.00 & 0.01 \\
\hline \multirow[t]{3}{*}{ MSI2 } & 225240_PM_s_at & musashi homolog 2 (Drosophila) & -1.17 & 0.00 & 0.00 \\
\hline & 217647_PM_at & & -1.17 & 0.00 & 0.00 \\
\hline & 236139_PM_at & & -1.17 & 0.00 & 0.00 \\
\hline KIAA0355 & 203288_PM_at & KIAA0355 & -1.17 & 0.00 & 0.00 \\
\hline FSCN1 & 210933_PM_s_at & fascin homolog 1 , actin-bundling $p$ & -1.17 & 0.00 & 0.00 \\
\hline \multirow[t]{2}{*}{ KIAA0494 } & 1559023_PM_a_at & KIAA0494 & -1.17 & 0.00 & 0.01 \\
\hline & 242089_PM_at & & -1.17 & 0.00 & 0.01 \\
\hline \multirow[t]{2}{*}{ XBP1 } & 242021_PM_at & X-box binding protein 1 & -1.17 & 0.00 & 0.00 \\
\hline & 215974_PM_at & & -1.17 & 0.00 & 0.00 \\
\hline LUC7L & 1557066_PM_at & LUC7-like (S. cerevisiae) & -1.17 & 0.00 & 0.00 \\
\hline ZNF330 & 209814_PM_at & zinc finger protein 330 & -1.17 & 0.00 & 0.01 \\
\hline \multirow[t]{2}{*}{ USP34 } & 233595_PM_at & ubiquitin specific peptidase 34 & -1.17 & 0.00 & 0.00 \\
\hline & 243249_PM_at & & -1.17 & 0.00 & 0.00 \\
\hline Cox18 & 227442_PM_at & cOX18 cytochrome c oxidase asser & -1.17 & 0.00 & 0.00 \\
\hline MIR1204 & 222087_PM_at & microRNA 1204 & -1.17 & 0.00 & 0.01 \\
\hline CNOT4 & 210866_PM_s_at & CCR4-NOT transcription complex, ! & -1.17 & 0.00 & 0.01 \\
\hline
\end{tabular}




\begin{tabular}{|c|c|c|c|c|c|}
\hline \multirow[t]{2}{*}{$\mathrm{COCH}$} & 1554241_PM_at & \multirow[t]{2}{*}{ coagulation factor $C$ homolog, cocl } & -1.17 & 0.00 & 0.01 \\
\hline & 1556818_PM_at & & -1.17 & 0.00 & 0.00 \\
\hline EVL & 227232_PM_at & Enah & -1.17 & 0.00 & 0.01 \\
\hline PTCD2 & 219658_PM_at & pentatricopeptide repeat domain : & -1.16 & 0.00 & 0.00 \\
\hline TRIB2 & 202479_PM_s_at & tribbles homolog 2 (Drosophila) & -1.16 & 0.00 & 0.01 \\
\hline ACAD10 & 237481_PM_at & acyl-CoA dehydrogenase family, $m$ & -1.16 & 0.00 & 0.01 \\
\hline PCSK7 & 232521_PM_at & proprotein convertase subtilisin & -1.16 & 0.00 & 0.00 \\
\hline OXNAD1 & 227686_PM_at & oxidoreductase NAD-binding doma & -1.16 & 0.00 & 0.01 \\
\hline ABLIM1 & 200965_PM_s_at & actin binding LIM protein 1 & -1.16 & 0.00 & 0.00 \\
\hline RIMKLB & 229344_PM_x_at & ribosomal modification protein rin & -1.16 & 0.00 & 0.00 \\
\hline DCAF8 & 243318_PM_at & DDB1 and CUL4 associated factor $\varepsilon$ & -1.16 & 0.00 & 0.00 \\
\hline TCF4 & 222146_PM_s_at & transcription factor 4 & -1.16 & 0.00 & 0.01 \\
\hline \multirow[t]{4}{*}{ SFSWAP } & 202773_PM_s_at & splicing factor, suppressor of whit $\epsilon$ & -1.16 & 0.00 & 0.00 \\
\hline & 243329_PM_at & & -1.16 & 0.00 & 0.00 \\
\hline & 242431_PM_at & & -1.16 & 0.00 & 0.00 \\
\hline & 239160_PM_at & & -1.16 & 0.00 & 0.01 \\
\hline \multirow[t]{2}{*}{ KTN1 } & 200914_PM_x_at & kinectin 1 (kinesin receptor) & -1.16 & 0.00 & 0.00 \\
\hline & 234153_PM_at & & -1.16 & 0.00 & 0.00 \\
\hline AGAP4 & 1565620_PM_at & ArfGAP with GTPase domain, anky & -1.16 & 0.00 & 0.01 \\
\hline HEATR1 & 232414_PM_at & HEAT repeat containing 1 & -1.16 & 0.00 & 0.00 \\
\hline PSMG4 & 228217_PM_s_at & proteasome (prosome, macropain' & -1.16 & 0.00 & 0.00 \\
\hline YME1L1 & 224343_PM_x_at & YME1-like 1 (S. cerevisiae) & -1.16 & 0.00 & 0.00 \\
\hline AGPAT4 & 219693_PM_at & 1-acylglycerol-3-phosphate O-acyli & -1.16 & 0.00 & 0.01 \\
\hline \multirow[t]{2}{*}{ ZMYND8 } & 230533_PM_at & zinc finger, MYND-type containing & -1.16 & 0.00 & 0.01 \\
\hline & 232700_PM_at & & -1.16 & 0.00 & 0.01 \\
\hline \multirow[t]{2}{*}{ GPR157 } & 227970_PM_at & G protein-coupled receptor 157 & -1.16 & 0.00 & 0.01 \\
\hline & 222306_PM_at & & -1.16 & 0.00 & 0.00 \\
\hline \multirow[t]{2}{*}{ TRIB2 } & 202478_PM_at & tribbles homolog 2 (Drosophila) & -1.16 & 0.00 & 0.01 \\
\hline & 228156_PM_at & & -1.16 & 0.00 & 0.00 \\
\hline \multirow[t]{2}{*}{ FBXO21 } & 212229_PM_s_at & F-box protein 21 & -1.16 & 0.00 & 0.00 \\
\hline & 242194_PM_at & & -1.16 & 0.00 & 0.00 \\
\hline NOP14 & 214661_PM_s_at & NOP14 nucleolar protein homolog & -1.16 & 0.00 & 0.01 \\
\hline \multirow[t]{2}{*}{ NGDN } & 216263_PM_s_at & neuroguidin, EIF4E binding protein & -1.16 & 0.00 & 0.01 \\
\hline & 1562673_PM_at & & -1.16 & 0.00 & 0.00 \\
\hline SIKE1 & 221705_PM_s_at & suppressor of IKBKE 1 & -1.16 & 0.00 & 0.01 \\
\hline CCDC50 & 225331_PM_at & coiled-coil domain containing 50 & -1.16 & 0.00 & 0.01 \\
\hline \multirow[t]{2}{*}{ HSPH1 } & 208744_PM_x_at & heat shock $105 \mathrm{kDa}$ & -1.16 & 0.00 & 0.00 \\
\hline & 238645_PM_at & & -1.16 & 0.00 & 0.01 \\
\hline C4orf29 & 219980_PM_at & chromosome 4 open reading framı & -1.16 & 0.00 & 0.00 \\
\hline ZNF747 & 238606_PM_at & zinc finger protein 747 & -1.16 & 0.00 & 0.00 \\
\hline SERBP1 & 209669_PM_s_at & SERPINE1 mRNA binding protein 1 & -1.16 & 0.00 & 0.01 \\
\hline NAPEPLD & 238722_PM_x_at & $\mathrm{N}$-acyl phosphatidylethanolamine & -1.16 & 0.00 & 0.00 \\
\hline U2SURP & 236431_PM_at & U2 snRNP-associated SURP domair & -1.16 & 0.00 & 0.01 \\
\hline \multirow[t]{2}{*}{ DENR } & 238982_PM_at & density-regulated protein & -1.16 & 0.00 & 0.00 \\
\hline & 236168_PM_at & & -1.16 & 0.00 & 0.00 \\
\hline ELP2 & 235623_PM_at & elongation protein 2 homolog (S. c & -1.15 & 0.00 & 0.01 \\
\hline $\mathrm{CIRH} 1 \mathrm{~A}$ & 228179_PM_at & cirrhosis, autosomal recessive $1 \mathrm{~A}$ & -1.15 & 0.00 & 0.00 \\
\hline LOC100505549 & C 238055_PM_at & uncharacterized LOC100505549 & -1.15 & 0.00 & 0.00 \\
\hline TMEM111 & 215250_PM_at & Transmembrane protein 111 & -1.15 & 0.00 & 0.01 \\
\hline COBLL1 & 215393_PM_s_at & COBL-like 1 & -1.15 & 0.00 & 0.01 \\
\hline
\end{tabular}




\begin{tabular}{|c|c|c|c|c|c|}
\hline SNHG12 & 223773_PM_s_at & small nucleolar RNA host gene 121 & -1.15 & 0.00 & 0.00 \\
\hline \multirow{2}{*}{ ATF7IP2 } & 219870_PM_at & activating transcription factor 7 int & -1.15 & 0.00 & 0.01 \\
\hline & 243217_PM_at & & -1.15 & 0.00 & 0.01 \\
\hline \multirow[t]{2}{*}{ ZNF224 } & 232427_PM_at & Zinc finger protein 224 & -1.15 & 0.00 & 0.00 \\
\hline & 1556479_PM_at & & -1.15 & 0.00 & 0.01 \\
\hline \multirow[t]{2}{*}{ LOC100507495 } & 5236520_PM_at & Uncharacterized LOC100507495 & -1.15 & 0.00 & 0.01 \\
\hline & 240121_PM_x_at & & -1.15 & 0.00 & 0.00 \\
\hline \multirow[t]{2}{*}{ NOP14-AS1 } & 214123_PM_s_at & NOP14 antisense RNA 1 (non-protı & -1.15 & 0.00 & 0.01 \\
\hline & 236586_PM_at & & -1.15 & 0.00 & 0.01 \\
\hline ZNF830 & 226315_PM_at & zinc finger protein 830 & -1.15 & 0.00 & 0.01 \\
\hline NASP & 201969_PM_at & nuclear autoantigenic sperm prote & -1.15 & 0.00 & 0.00 \\
\hline \multicolumn{2}{|c|}{ LOC100132707 1558527_PM_at } & uncharacterized LOC100132707 & -1.15 & 0.00 & 0.00 \\
\hline FAM60A & 223038_PM_s_at & family with sequence similarity 60 , & -1.15 & 0.00 & 0.01 \\
\hline AQP3 & 39248_PM_at & aquaporin 3 (Gill blood group) & -1.15 & 0.00 & 0.01 \\
\hline ZDHHC14 & 219247_PM_s_s_at & zinc finger, DHHC-type containing & -1.15 & 0.00 & 0.00 \\
\hline \multirow[t]{3}{*}{ PCCB } & 1557502_PM_at & Propionyl CoA carboxylase, beta pr & -1.15 & 0.00 & 0.00 \\
\hline & 236699_PM_at & & -1.15 & 0.00 & 0.01 \\
\hline & 243091_PM_at & & -1.15 & 0.00 & 0.00 \\
\hline LUC7L & 1557067_PM_s_at & LUC7-like (S. cerevisiae) & -1.15 & 0.00 & 0.01 \\
\hline \multirow[t]{2}{*}{ ZBTB44 } & 220243_PM_at & zinc finger and BTB domain contair & -1.15 & 0.00 & 0.00 \\
\hline & 1556434_PM_at & & -1.15 & 0.00 & 0.00 \\
\hline NEK1 & 213328_PM_at & NIMA (never in mitosis gene a)-reli & -1.15 & 0.00 & 0.00 \\
\hline KCTD6 & 238077_PM_at & potassium channel tetramerisatior & -1.15 & 0.00 & 0.00 \\
\hline PNISR & 212177_PM_at & PNN-interacting serine & -1.15 & 0.00 & 0.00 \\
\hline \multirow[t]{2}{*}{ CSPP1 } & 220072_PM_at & centrosome and spindle pole assor & -1.15 & 0.00 & 0.01 \\
\hline & 216440_PM_at & & -1.15 & 0.00 & 0.01 \\
\hline PMS2L2 & 214757_PM_at & postmeiotic segregation increased & -1.15 & 0.00 & 0.01 \\
\hline FOXK1 & 226715_PM_at & forkhead box K1 & -1.15 & 0.00 & 0.00 \\
\hline RNPC3 & 226999_PM_at & RNA-binding region (RNP1, RRM) C & -1.15 & 0.00 & 0.01 \\
\hline KLHL28 & 235727_PM_at & kelch-like 28 (Drosophila) & -1.15 & 0.00 & 0.00 \\
\hline \multirow[t]{4}{*}{ SYNPO } & 202796_PM_at & synaptopodin & -1.15 & 0.00 & 0.00 \\
\hline & 234164_PM_at & & -1.15 & 0.00 & 0.00 \\
\hline & 1559054_PM_a_at & & -1.15 & 0.00 & 0.00 \\
\hline & 226773_PM_at & & -1.15 & 0.00 & 0.00 \\
\hline CSTF3 & 229666_PM_S_at & cleavage stimulation factor, 3 ' pre- & -1.15 & 0.00 & 0.00 \\
\hline BOLA2 & 231500_PM_s_at & BolA homolog 2 (E. coli) & -1.15 & 0.00 & 0.00 \\
\hline SPAG8 & 206816_PM_s_at & sperm associated antigen 8 & -1.14 & 0.00 & 0.00 \\
\hline PDSS1 & 236298_PM_at & Prenyl (decaprenyl) diphosphate s! & -1.14 & 0.00 & 0.01 \\
\hline NPIPL3 & 215920_PM_s_at & nuclear pore complex interacting $\mathrm{k}$ & -1.14 & 0.00 & 0.00 \\
\hline CBFA2T2 & 238549_PM_at & core-binding factor, runt domain, a & -1.14 & 0.00 & 0.01 \\
\hline KCMF1 & 242887_PM_at & potassium channel modulatory fac & -1.14 & 0.00 & 0.00 \\
\hline RBM17 & 224781_PM_s_at & RNA binding motif protein 17 & -1.14 & 0.00 & 0.01 \\
\hline \multirow[t]{2}{*}{ BOLA2 } & 241644_PM_at & bolA homolog 2 (E. coli) & -1.14 & 0.00 & 0.01 \\
\hline & 1553547_PM_at & & -1.14 & 0.00 & 0.01 \\
\hline GAR1 & 219110_PM_at & GAR1 ribonucleoprotein homolog & -1.14 & 0.00 & 0.00 \\
\hline \multirow{2}{*}{ CTNNB1 } & 223679_PM_at & catenin (cadherin-associated prot $€$ & -1.14 & 0.00 & 0.00 \\
\hline & 1556322_PM_a_at & & -1.14 & 0.00 & 0.01 \\
\hline RBM4 & 213718_PM_at & RNA binding motif protein 4 & -1.14 & 0.00 & 0.00 \\
\hline \multirow[t]{2}{*}{ RBM19 } & 206019_PM_at & RNA binding motif protein 19 & -1.14 & 0.00 & 0.01 \\
\hline & 244826_PM_at & & -1.14 & 0.00 & 0.00 \\
\hline
\end{tabular}




\begin{tabular}{|c|c|c|c|c|c|}
\hline RAD52 & 205647_PM_at & RAD52 homolog (S. cerevisiae) & -1.14 & 0.00 & 0.01 \\
\hline RGS10 & 204316_PM_at & regulator of G-protein signaling 10 & -1.14 & 0.00 & 0.00 \\
\hline DNAJC16 & 212908_PM_at & DnaJ (Hsp40) homolog, subfamily ( & -1.14 & 0.00 & 0.00 \\
\hline \multirow[t]{2}{*}{ VAV2 } & 226063_PM_at & vav 2 guanine nucleotide exchang $€$ & -1.14 & 0.00 & 0.00 \\
\hline & 236607_PM_at & & -1.14 & 0.00 & 0.01 \\
\hline KDELR2 & 200700_PM_s_at & KDEL (Lys-Asp-Glu-Leu) endoplasm & -1.14 & 0.00 & 0.00 \\
\hline UCHL5 & 220083_PM_x_at & ubiquitin carboxyl-terminal hydroli & -1.14 & 0.00 & 0.01 \\
\hline TSR2 & 213079_PM_at & TSR2, 20S rRNA accumulation, hon & -1.14 & 0.00 & 0.01 \\
\hline CNOT7 & 233019_PM_at & CCR4-NOT transcription complex, & -1.14 & 0.00 & 0.01 \\
\hline \multirow[t]{2}{*}{ LUC7L } & 223546_PM_x_at & LUC7-like (S. cerevisiae) & -1.14 & 0.00 & 0.00 \\
\hline & 242725_PM_at & & -1.14 & 0.00 & 0.01 \\
\hline AMN & 1563792_PM_at & amnionless homolog (mouse) & -1.14 & 0.00 & 0.00 \\
\hline RPP14 & 204245_PM_s_at & ribonuclease $\mathrm{P}$ & -1.14 & 0.00 & 0.00 \\
\hline TERF1 & 203448_PM_s_at & telomeric repeat binding factor $(\mathrm{N})$ & -1.13 & 0.00 & 0.00 \\
\hline LOC285033 & 235584_PM_at & uncharacterized LOC285033 & -1.13 & 0.00 & 0.00 \\
\hline TNRC6A & 234734_PM_s_at & trinucleotide repeat containing $6 \mathrm{~A}$ & -1.13 & 0.00 & 0.00 \\
\hline \multirow[t]{2}{*}{ FUS } & 217370_PM_x_at & fused in sarcoma & -1.13 & 0.00 & 0.01 \\
\hline & 233235_PM_x_at & & -1.13 & 0.00 & 0.01 \\
\hline \multirow[t]{2}{*}{ MRPS5 } & 224877_PM_s_at & mitochondrial ribosomal protein $\mathrm{S}$ ! & -1.13 & 0.00 & 0.00 \\
\hline & 217464_PM_at & & -1.13 & 0.00 & 0.01 \\
\hline RBM25 & 212027_PM_at & RNA binding motif protein 25 & -1.13 & 0.00 & 0.01 \\
\hline \multirow[t]{2}{*}{ RCL1 } & 222666_PM_s_at & RNA terminal phosphate cyclase-lil & -1.13 & 0.00 & 0.00 \\
\hline & 240241_PM_at & & -1.13 & 0.00 & 0.01 \\
\hline CCNL2 & 221427_PM_s_at & cyclin L2 & -1.13 & 0.00 & 0.00 \\
\hline TNRC6B & 229036_PM_at & trinucleotide repeat containing $6 \mathrm{~B}$ & -1.13 & 0.00 & 0.01 \\
\hline ZBTB4 & 225629_PM_s_at & zinc finger and BTB domain contair & -1.13 & 0.00 & 0.00 \\
\hline LUC7L3 & 208835_PM_s_at & LUC7-like 3 (S. cerevisiae) & -1.13 & 0.00 & 0.00 \\
\hline GNAS & 228173_PM_at & GNAS complex locus & -1.13 & 0.00 & 0.01 \\
\hline KLHL26 & 219354_PM_at & kelch-like 26 (Drosophila) & -1.13 & 0.00 & 0.00 \\
\hline SIM2 & 206558_PM_at & single-minded homolog 2 (Drosopl & -1.13 & 0.00 & 0.01 \\
\hline LOC10065304 & 214107_PM_x_at & uncharacterized LOC100653042 & -1.13 & 0.00 & 0.00 \\
\hline BICD1 & 204741_PM_at & bicaudal D homolog 1 (Drosophila) & -1.13 & 0.00 & 0.00 \\
\hline SP140L & 223934_PM_at & SP140 nuclear body protein-like & -1.13 & 0.00 & 0.00 \\
\hline CCAR1 & 224736_PM_at & cell division cycle and apoptosis re & -1.13 & 0.00 & 0.00 \\
\hline AKIRIN1 & 222458_PM_s_at & akirin 1 & -1.13 & 0.00 & 0.00 \\
\hline KDSR & 202419_PM_at & 3-ketodihydrosphingosine reducta & -1.13 & 0.00 & 0.01 \\
\hline LEPROTL1 & 202595_PM_s_at & leptin receptor overlapping transcl & -1.13 & 0.00 & 0.00 \\
\hline UNKL & 221064_PM_s_at & unkempt homolog (Drosophila)-lik & -1.13 & 0.00 & 0.01 \\
\hline RBM25 & 1557081_PM_at & RNA binding motif protein 25 & -1.13 & 0.00 & 0.01 \\
\hline SLC25A14 & 204587_PM_at & solute carrier family 25 (mitochonc & -1.13 & 0.00 & 0.00 \\
\hline \multirow[t]{2}{*}{ ZNF493 } & 240155_PM_x_at & zinc finger protein 493 & -1.12 & 0.00 & 0.01 \\
\hline & 240128_PM_at & & -1.12 & 0.00 & 0.01 \\
\hline SDC2 & 212157_PM_at & syndecan 2 & -1.12 & 0.00 & 0.01 \\
\hline UBE3B & 212403_PM_at & ubiquitin protein ligase E3B & -1.12 & 0.00 & 0.00 \\
\hline PATZ1 & 209431_PM_s_at & POZ (BTB) and AT hook containing & -1.12 & 0.00 & 0.01 \\
\hline SUGP2 & 64371_PM_at & SURP and G patch domain containi & -1.12 & 0.00 & 0.01 \\
\hline PTBP1 & 226491_PM_x_at & Polypyrimidine tract binding prote & -1.12 & 0.00 & 0.00 \\
\hline \multirow[t]{2}{*}{ TMEM101 } & 225004_PM_at & transmembrane protein 101 & -1.12 & 0.00 & 0.00 \\
\hline & 230461_PM_s_at & & -1.12 & 0.00 & 0.01 \\
\hline TRIT1 & 218617_PM_at & tRNA isopentenyltransferase 1 & -1.12 & 0.00 & 0.01 \\
\hline
\end{tabular}




\begin{tabular}{|c|c|c|c|c|c|}
\hline AQP11 & 229526_PM_at & aquaporin 11 & -1.12 & 0.00 & 0.00 \\
\hline HSPG2 & 201655_PM_s_at & heparan sulfate proteoglycan 2 & -1.12 & 0.00 & 0.00 \\
\hline POLR3A & 231763_PM_at & polymerase (RNA) III (DNA directer & -1.12 & 0.00 & 0.01 \\
\hline RBMX2 & 204097_PM_s_at & RNA binding motif protein, $\mathrm{X}$-linke & -1.12 & 0.00 & 0.00 \\
\hline RCN1 & 201063_PM_at & reticulocalbin 1, EF-hand calcium $k$ & -1.12 & 0.00 & 0.01 \\
\hline LOC440434 & 215090_PM_x_at & aminopeptidase puromycin sensiti & -1.12 & 0.00 & 0.01 \\
\hline ZNF498 & 228138_PM_at & zinc finger protein 498 & -1.12 & 0.00 & 0.00 \\
\hline TCF3 & 210776_PM_x_at & transcription factor 3 (E2A immun & -1.11 & 0.00 & 0.01 \\
\hline RARG & 204189_PM_at & retinoic acid receptor, gamma & -1.11 & 0.00 & 0.01 \\
\hline FAM165B & 228239_PM_at & family with sequence similarity $16 !$ & -1.11 & 0.00 & 0.01 \\
\hline EXOSC9 & 205061_PM_s_at & exosome component 9 & -1.11 & 0.00 & 0.01 \\
\hline TGIF2 & 216262_PM_s_at & TGFB-induced factor homeobox 2 & -1.11 & 0.00 & 0.00 \\
\hline SFRP5 & 207468_PM_s_at & secreted frizzled-related protein 5 & -1.11 & 0.00 & 0.01 \\
\hline LUC7L & 220143_PM_x_at & LUC7-like (S. cerevisiae) & -1.11 & 0.00 & 0.01 \\
\hline ANKRD11 & 238538_PM_at & ankyrin repeat domain 11 & -1.11 & 0.00 & 0.00 \\
\hline TEX10 & 218104_PM_at & testis expressed 10 & -1.11 & 0.00 & 0.01 \\
\hline ZBTB43 & 231393_PM_x_at & Zinc finger and BTB domain contaiı & -1.11 & 0.00 & 0.00 \\
\hline ZNF562 & 219163_PM_at & zinc finger protein 562 & -1.11 & 0.00 & 0.00 \\
\hline $\mathrm{CHD} 2$ & 1554015_PM_a_at & chromodomain helicase DNA bindi & -1.11 & 0.00 & 0.01 \\
\hline RNF115 & 212742_PM_at & ring finger protein 115 & -1.11 & 0.00 & 0.01 \\
\hline LOC10027183 & E208118_PM_x_at & smg-1 homolog, phosphatidylinosi & -1.11 & 0.00 & 0.00 \\
\hline CBFA2T2 & 207625_PM_s_at & core-binding factor, runt domain, a & -1.11 & 0.00 & 0.00 \\
\hline PRPF31 & 214380_PM_at & PRP31 pre-mRNA processing facto & -1.11 & 0.00 & 0.01 \\
\hline СCT5 & 208696_PM_at & chaperonin containing TCP1, subur & -1.11 & 0.00 & 0.01 \\
\hline SDK2 & 234073_PM_at & Sidekick cell adhesion molecule 2 & -1.11 & 0.00 & 0.01 \\
\hline RARRES1 & 206391_PM_at & retinoic acid receptor responder ( $\mathrm{t}$ & -1.11 & 0.00 & 0.00 \\
\hline TCF20 & 212931_PM_at & transcription factor 20 (AR1) & -1.11 & 0.00 & 0.00 \\
\hline \multirow[t]{2}{*}{ WIPI2 } & 202031_PM_s_at & WD repeat domain, phosphoinosit & -1.11 & 0.00 & 0.00 \\
\hline & 228638_PM_at & & -1.11 & 0.00 & 0.01 \\
\hline FAM122A & 226443_PM_at & family with sequence similarity 12 : & -1.11 & 0.00 & 0.01 \\
\hline LIMS2 & 220765_PM_s_at & LIM and senescent cell antigen-lik€ & -1.11 & 0.00 & 0.01 \\
\hline QRICH1 & 229806_PM_at & Glutamine-rich 1 & -1.11 & 0.00 & 0.00 \\
\hline RAB3GAP1 & 213531_PM_s_at & RAB3 GTPase activating protein su & -1.11 & 0.00 & 0.01 \\
\hline MAP3К13 & 206249_PM_at & mitogen-activated protein kinase $k$ & -1.11 & 0.00 & 0.00 \\
\hline BANF1 & 210125_PM_s_at & barrier to autointegration factor 1 & -1.11 & 0.00 & 0.01 \\
\hline MRPL49 & 201717_PM_at & mitochondrial ribosomal protein L، & -1.11 & 0.00 & 0.01 \\
\hline AIPL1 & 235731_PM_at & aryl hydrocarbon receptor interact & -1.10 & 0.00 & 0.01 \\
\hline C12orf76 & 226583_PM_at & chromosome 12 open reading fran & -1.10 & 0.00 & 0.01 \\
\hline ARHGEF7 & 202548_PM_S_at & Rho guanine nucleotide exchange & -1.10 & 0.00 & 0.00 \\
\hline STK35 & 225649_PM_s_at & serine & -1.10 & 0.00 & 0.00 \\
\hline TBP & 203135_PM_at & TATA box binding protein & -1.10 & 0.00 & 0.01 \\
\hline SMU1 & 222618_PM_at & smu- 1 suppressor of mec- 8 and un & -1.10 & 0.00 & 0.01 \\
\hline SLC35A2 & 209326_PM_at & solute carrier family 35 (UDP-galac & -1.10 & 0.00 & 0.01 \\
\hline GSX1 & 1555676_PM_s_at & GS homeobox 1 & -1.10 & 0.00 & 0.01 \\
\hline NDFIP1 & 222422_PM_s_at & Nedd4 family interacting protein 1 & -1.10 & 0.00 & 0.01 \\
\hline SFPQ & 214016_PM_s_at & splicing factor proline & -1.10 & 0.00 & 0.01 \\
\hline CNTN1 & 211203_PM_s_at & contactin 1 & -1.10 & 0.00 & 0.01 \\
\hline $\mathrm{MB}$ & 204179_PM_at & myoglobin & -1.10 & 0.00 & 0.01 \\
\hline FAM192A & 224248_PM_x_at & family with sequence similarity $19:$ & -1.10 & 0.00 & 0.00 \\
\hline GTF2H1 & 202451_PM_at & general transcription factor $\mathrm{IIH}$, po & -1.10 & 0.00 & 0.01 \\
\hline
\end{tabular}




\begin{tabular}{|c|c|c|c|c|c|}
\hline APH1A & 218389_PM_s_at & anterior pharynx defective 1 homc & -1.09 & 0.00 & 0.01 \\
\hline MTMR1 & 216095_PM_x_at & myotubularin related protein 1 & -1.09 & 0.00 & 0.00 \\
\hline DERL2 & 226719_PM_at & derlin 2 & -1.09 & 0.00 & 0.00 \\
\hline ZNF212 & 203985_PM_at & zinc finger protein 212 & -1.09 & 0.00 & 0.01 \\
\hline MTMR1 & 213511_PM_s_at & myotubularin related protein 1 & -1.09 & 0.00 & 0.01 \\
\hline SLC35E1 & 227518_PM_at & solute carrier family 35 , member $\mathrm{E}$ & -1.09 & 0.00 & 0.01 \\
\hline SRSF5 & 212266_PM_s_at & serine & -1.09 & 0.00 & 0.01 \\
\hline FGD2 & 1553906_PM_s_at & FYVE, RhoGEF and PH domain cont & -1.09 & 0.00 & 0.01 \\
\hline RPL22 & 214042_PM_s_at & ribosomal protein L22 & -1.08 & 0.00 & 0.00 \\
\hline NR2E3 & 208385_PM_at & nuclear receptor subfamily 2 , grou & -1.08 & 0.00 & 0.02 \\
\hline NONO & 208698_PM_s_at & non-POU domain containing, octar & -1.08 & 0.00 & 0.01 \\
\hline FAM192A & 223406_PM_x_at & family with sequence similarity $19:$ & -1.08 & 0.00 & 0.01 \\
\hline OR8G2 & 1567056_PM_at & olfactory receptor, family 8 , subfar & -1.08 & 0.00 & 0.02 \\
\hline R3HDM2 & 203831_PM_at & R3H domain containing 2 & -1.07 & 0.00 & 0.01 \\
\hline HNRNPD & 221481_PM_x_at & heterogeneous nuclear ribonucleo & -1.07 & 0.00 & 0.01 \\
\hline SRSF1 & 211784_PM_s_at & serine & -1.07 & 0.00 & 0.01 \\
\hline PSMB4 & 202243_PM_s_at & proteasome (prosome, macropain' & -1.06 & 0.00 & 0.01 \\
\hline \multirow[t]{2}{*}{ PSMB4 } & 228204_PM_at & Proteasome (prosome, macropain' & -1.05 & 0.00 & 0.02 \\
\hline & 214182_PM_at & & -1.04 & 0.00 & 0.03 \\
\hline АСТВ & 200801_PM_x_at & actin, beta & 1.03 & 0.00 & 0.05 \\
\hline АСТВ & 213867_PM_x_at & actin, beta & 1.03 & 0.00 & 0.02 \\
\hline HBB & 211696_PM_x_at & hemoglobin, beta & 1.03 & 0.00 & 0.05 \\
\hline MYL12A & 201318_PM_s_at & myosin, light chain $12 \mathrm{~A}$, regulatory & 1.04 & 0.00 & 0.04 \\
\hline АСТВ & AFFX-HSAC07/X0035 & actin, beta & 1.04 & 0.00 & 0.01 \\
\hline TMEFF2 & 224321_PM_at & transmembrane protein with EGF- & 1.06 & 0.00 & 0.02 \\
\hline ATP6V0E1 & 201172_PM_x_at & ATPase, $\mathrm{H}+$ transporting, lysosoma & 1.06 & 0.00 & 0.01 \\
\hline CAP1 & 200625_PM_s_at & CAP, adenylate cyclase-associated & 1.06 & 0.00 & 0.01 \\
\hline YWHAZ & 200639_PM_s_at & tyrosine 3-monooxygenase & 1.07 & 0.00 & 0.00 \\
\hline \multicolumn{2}{|c|}{ LOC100652765 201171_PM_at } & uncharacterized LOC100652765 & 1.07 & 0.00 & 0.01 \\
\hline LYZ & 213975_PM_s_at & lysozyme & 1.08 & 0.00 & 0.00 \\
\hline \multirow[t]{2}{*}{ CCL14 } & 210390_PM_s_at & chemokine (C-C motif) ligand 14 & 1.08 & 0.00 & 0.01 \\
\hline & 1566342_PM_at & & 1.08 & 0.00 & 0.00 \\
\hline BTG4 & 1554362_PM_at & B-cell translocation gene 4 & 1.08 & 0.00 & 0.01 \\
\hline BRK1 & 224023_PM_s_sat & BRICK1, SCAR & 1.08 & 0.00 & 0.02 \\
\hline FTH1P5 & 211628_PM_x_at & ferritin, heavy polypeptide 1 pseuc & 1.08 & 0.00 & 0.02 \\
\hline \multirow[t]{2}{*}{ CDC42SE1 } & 218157_PM_x_at & CDC42 small effector 1 & 1.08 & 0.00 & 0.00 \\
\hline & AFFX-BioDn-3_at & & 1.08 & 0.00 & 0.01 \\
\hline B2M & 201891_PM_s_at & beta-2-microglobulin & 1.08 & 0.00 & 0.00 \\
\hline LINC00340 & 1562469_PM_at & long intergenic non-protein coding & 1.08 & 0.00 & 0.01 \\
\hline BIRC6 & 224635_PM_s_at & baculoviral IAP repeat containing $\epsilon$ & 1.08 & 0.00 & 0.02 \\
\hline PSMD7 & 201705_PM_at & proteasome (prosome, macropain' & 1.08 & 0.00 & 0.01 \\
\hline TMEM59 & 200620_PM_at & transmembrane protein 59 & 1.09 & 0.00 & 0.00 \\
\hline MORF4L1 & 221381_PM_s_at & mortality factor 4 like 1 & 1.09 & 0.00 & 0.00 \\
\hline LOC440944 & 1557357_PM_at & uncharacterized LOC440944 & 1.09 & 0.00 & 0.00 \\
\hline \multirow[t]{2}{*}{ SPG11 } & 203513_PM_at & spastic paraplegia 11 (autosomal $r$ & 1.09 & 0.00 & 0.01 \\
\hline & \multicolumn{2}{|c|}{ AFFX-r2-Ec-bioD-3_at } & 1.09 & 0.00 & 0.01 \\
\hline RAB11A & 200863_PM_s_at & RAB11A, member RAS oncogene $f_{c}$ & 1.09 & 0.00 & 0.01 \\
\hline H2AFZ & 213911_PM_s_at & H2A histone family, member Z & 1.09 & 0.00 & 0.01 \\
\hline MBD4 & 214047_PM_s_at & methyl-CpG binding domain protei & 1.09 & 0.00 & 0.00 \\
\hline PPP2CA & 208652_PM_at & protein phosphatase 2 , catalytic sı & 1.09 & 0.00 & 0.01 \\
\hline
\end{tabular}




\begin{tabular}{|c|c|c|c|c|c|}
\hline NBPF10 & 212854_PM_x_at & neuroblastoma breakpoint family, & 1.09 & 0.00 & 0.02 \\
\hline SRGN & 201859_PM_at & serglycin & 1.10 & 0.00 & 0.01 \\
\hline \multirow[t]{2}{*}{ IDS } & 212221_PM_x_at & iduronate 2-sulfatase & 1.10 & 0.00 & 0.02 \\
\hline & 216420_PM_at & & 1.10 & 0.00 & 0.01 \\
\hline SETX & 201964_PM_at & senataxin & 1.10 & 0.00 & 0.02 \\
\hline \multirow[t]{2}{*}{ EVI2B } & 211742_PM_s_at & ecotropic viral integration site $2 B$ & 1.10 & 0.00 & 0.01 \\
\hline & 1569812_PM_at & & 1.10 & 0.00 & 0.01 \\
\hline MAOB & 1561009_PM_at & Monoamine oxidase B & 1.10 & 0.00 & 0.00 \\
\hline AK2 & 212174_PM_at & adenylate kinase 2 & 1.10 & 0.00 & 0.02 \\
\hline PLEKHB2 & 224121_PM_x_at & pleckstrin homology domain conta & 1.10 & 0.00 & 0.01 \\
\hline \multirow[t]{2}{*}{ MCU } & 225320_PM_at & mitochondrial calcium uniporter & 1.10 & 0.00 & 0.02 \\
\hline & AFFX-r2-Ec-bioD-5_a & & 1.10 & 0.00 & 0.00 \\
\hline CFTR & 234702_PM_x_at & cystic fibrosis transmembrane con & 1.11 & 0.00 & 0.01 \\
\hline \multicolumn{2}{|c|}{ LOC100507699202395_PM_at } & vesicle-fusing ATPase-like & 1.11 & 0.00 & 0.01 \\
\hline $\mathrm{H} 2 \mathrm{AFZ}$ & 200853_PM_at & H2A histone family, member Z & 1.11 & 0.00 & 0.01 \\
\hline RPS6KA3 & 203843_PM_at & ribosomal protein $\mathrm{S} 6$ kinase, $90 \mathrm{kDi}$ & 1.11 & 0.00 & 0.01 \\
\hline MORF4L1 & 224561_PM_s_at & mortality factor 4 like 1 & 1.11 & 0.00 & 0.00 \\
\hline PPCS & 218341_PM_at & phosphopantothenoylcysteine syn & 1.11 & 0.00 & 0.01 \\
\hline \multirow[t]{2}{*}{ OSBPL8 } & 212582_PM_at & oxysterol binding protein-like 8 & 1.11 & 0.00 & 0.01 \\
\hline & 1569772_PM_x_at & & 1.11 & 0.00 & 0.01 \\
\hline TINF2 & 224809_PM_x_at & TERF1 (TRF1)-interacting nuclear fi & 1.11 & 0.00 & 0.01 \\
\hline ATP6 & 242131_PM_at & ATP synthase F0 subunit 6 & 1.12 & 0.00 & 0.02 \\
\hline PICALM & 212506_PM_at & phosphatidylinositol binding clathr & 1.12 & 0.00 & 0.01 \\
\hline \multirow[t]{2}{*}{ ASAP1 } & 224791_PM_at & ArfGAP with SH3 domain, ankyrin I & 1.12 & 0.00 & 0.01 \\
\hline & 234371_PM_at & & 1.12 & 0.00 & 0.01 \\
\hline FGR & 208438_PM_s_at & Gardner-Rasheed feline sarcoma v & 1.12 & 0.00 & 0.01 \\
\hline MYL12B & 221474_PM_at & myosin, light chain $12 B$, regulatory & 1.12 & 0.00 & 0.00 \\
\hline LOC400590 & 1560992_PM_at & uncharacterized LOC400590 & 1.12 & 0.00 & 0.00 \\
\hline CHMP3 & 217837_PM_s_at & charged multivesicular body prote & 1.12 & 0.00 & 0.00 \\
\hline USP8 & 229501_PM_s_at & ubiquitin specific peptidase 8 & 1.12 & 0.00 & 0.00 \\
\hline MYL12A & 201319_PM_at & myosin, light chain $12 \mathrm{~A}$, regulatory & 1.12 & 0.00 & 0.00 \\
\hline BID & 227143_PM_s_at & $\mathrm{BH} 3$ interacting domain death ago & 1.13 & 0.00 & 0.01 \\
\hline UBQLN1 & 222990_PM_at & ubiquilin 1 & 1.13 & 0.00 & 0.02 \\
\hline \multicolumn{2}{|c|}{ LOC100128909236008_PM_at } & uncharacterized LOC100128909 & 1.13 & 0.00 & 0.01 \\
\hline \multirow[t]{2}{*}{ SFN } & 33323_PM_r_at & stratifin & 1.13 & 0.00 & 0.01 \\
\hline & 241617_PM_x_at & & 1.13 & 0.00 & 0.01 \\
\hline SCP2 & 211733_PM_x_at & sterol carrier protein 2 & 1.14 & 0.00 & 0.01 \\
\hline CENPBD1 & 223728_PM_at & CENPB DNA-binding domains cont & 1.14 & 0.00 & 0.01 \\
\hline STAG1 & 202294_PM_at & stromal antigen 1 & 1.14 & 0.00 & 0.01 \\
\hline PRELID1 & 223032_PM_x_at & PRELI domain containing 1 & 1.14 & 0.00 & 0.02 \\
\hline MAPK1 & 212271_PM_at & mitogen-activated protein kinase 1 & 1.14 & 0.00 & 0.01 \\
\hline \multicolumn{2}{|c|}{ LOC100507507 241353_PM_S_at } & uncharacterized LOC100507507 & 1.14 & 0.00 & 0.01 \\
\hline CFLAR & 237367_PM_x_at & CASP8 and FADD-like apoptosis rę & 1.14 & 0.00 & 0.01 \\
\hline GNAQ & 224861_PM_at & guanine nucleotide binding proteir & 1.14 & 0.00 & 0.01 \\
\hline NT5C2 & 209155_PM_s_at & 5'-nucleotidase, cytosolic II & 1.15 & 0.00 & 0.00 \\
\hline \multirow[t]{2}{*}{ PPP2R5A } & 202187_PM_s_at & protein phosphatase 2 , regulatory & 1.15 & 0.00 & 0.01 \\
\hline & 1564121_PM_at & & 1.15 & 0.00 & 0.01 \\
\hline SNTB2 & 226685_PM_at & syntrophin, beta 2 (dystrophin-ass & 1.15 & 0.00 & 0.02 \\
\hline DAPP1 & 219290_PM_x_at & dual adaptor of phosphotyrosine a & 1.15 & 0.00 & 0.00 \\
\hline HBXIP & 202299_PM_s_at & hepatitis $B$ virus $x$ interacting prot $\epsilon$ & 1.15 & 0.00 & 0.00 \\
\hline
\end{tabular}




\begin{tabular}{|c|c|c|c|c|c|}
\hline \multirow[t]{2}{*}{ TNFSF14 } & 233935_PM_at & \multirow[t]{2}{*}{ Tumor necrosis factor (ligand) sup } & 1.15 & 0.00 & 0.02 \\
\hline & 1555845_PM_at & & 1.16 & 0.00 & 0.02 \\
\hline NSMAF & 203269_PM_at & neutral sphingomyelinase (N-SMas & 1.16 & 0.00 & 0.00 \\
\hline LYST & 203518_PM_at & Iysosomal trafficking regulator & 1.16 & 0.00 & 0.02 \\
\hline \multirow[t]{2}{*}{ LINC00525 } & 1553708_PM_at & \multirow[t]{2}{*}{ long intergenic non-protein coding } & 1.16 & 0.00 & 0.01 \\
\hline & 242366_PM_at & & 1.16 & 0.00 & 0.00 \\
\hline PLXNC1 & 213241_PM_at & plexin $\mathrm{C} 1$ & 1.16 & 0.00 & 0.02 \\
\hline \multirow[t]{2}{*}{ RAB32 } & 228161_PM_at & \multirow[t]{2}{*}{ RAB32, member RAS oncogene far } & 1.16 & 0.00 & 0.01 \\
\hline & 229602_PM_at & & 1.16 & 0.00 & 0.01 \\
\hline \multirow[t]{2}{*}{ ALOX5 } & 204446_PM_s_at & \multirow[t]{2}{*}{ arachidonate 5-lipoxygenase } & 1.16 & 0.00 & 0.01 \\
\hline & 216580_PM_at & & 1.16 & 0.00 & 0.01 \\
\hline MYD88 & 209124_PM_at & myeloid differentiation primary re! & 1.16 & 0.00 & 0.00 \\
\hline IFNAR1 & 204191_PM_at & interferon (alpha, beta and omega & 1.16 & 0.00 & 0.02 \\
\hline CKLF & 219161_PM_s_at & chemokine-like factor & 1.16 & 0.00 & 0.01 \\
\hline CTSS & 202901_PM_x_at & cathepsin S & 1.16 & 0.00 & 0.00 \\
\hline ADAM8 & 205180_PM_s_at & ADAM metallopeptidase domain 8 & 1.16 & 0.00 & 0.02 \\
\hline HEBP2 & 203430_PM_at & heme binding protein 2 & 1.16 & 0.00 & 0.01 \\
\hline \multirow[t]{2}{*}{ ATP5J2 } & 202961_PM_s_at & \multirow[t]{2}{*}{ ATP synthase, $\mathrm{H}+$ transporting, mit } & 1.17 & 0.00 & 0.01 \\
\hline & 217352_PM_at & & 1.17 & 0.00 & 0.00 \\
\hline MYO5A & 204527_PM_at & myosin VA (heavy chain 12 , myoxir & 1.17 & 0.00 & 0.02 \\
\hline $\mathrm{SSH} 3$ & 219919_PM_s_at & slingshot homolog 3 (Drosophila) & 1.17 & 0.00 & 0.01 \\
\hline TMEM189 & 201003_PM_x_at & transmembrane protein 189 & 1.17 & 0.00 & 0.02 \\
\hline TPMT & 203671_PM_at & thiopurine S-methyltransferase & 1.17 & 0.00 & 0.01 \\
\hline CACNG8 & 231355_PM_at & calcium channel, voltage-depende & 1.17 & 0.00 & 0.00 \\
\hline S100A8 & 202917_PM_s_at & S100 calcium binding protein $\mathrm{A} 8$ & 1.17 & 0.00 & 0.01 \\
\hline PGM2 & 225367_PM_at & phosphoglucomutase 2 & 1.17 & 0.00 & 0.02 \\
\hline ALAS1 & 205633_PM_s_at & aminolevulinate, delta-, synthase 1 & 1.17 & 0.00 & 0.00 \\
\hline \multirow[t]{2}{*}{ TMEM33 } & 225492_PM_at & \multirow[t]{2}{*}{ transmembrane protein 33} & 1.17 & 0.00 & 0.02 \\
\hline & 229359_PM_at & & 1.17 & 0.00 & 0.01 \\
\hline AMPD3 & 207992_PM_s_at & adenosine monophosphate deami & 1.17 & 0.00 & 0.00 \\
\hline ARHGAP26 & 226576_PM_at & Rho GTPase activating protein 26 & 1.17 & 0.00 & 0.02 \\
\hline SLC25A20 & 203658_PM_at & solute carrier family 25 (carnitine & 1.17 & 0.00 & 0.01 \\
\hline SUSD1 & 226264_PM_at & sushi domain containing 1 & 1.17 & 0.00 & 0.01 \\
\hline DEFT1P & 1553518_PM_at & defensin, theta 1 pseudogene & 1.18 & 0.00 & 0.03 \\
\hline EEF1A1 & 1557120_PM_at & Eukaryotic translation elongation $f$ & 1.18 & 0.00 & 0.00 \\
\hline C9orf85 & 238912_PM_x_at & chromosome 9 open reading fram & 1.18 & 0.00 & 0.01 \\
\hline KCTD21 & 229873_PM_at & potassium channel tetramerisatior & 1.18 & 0.00 & 0.01 \\
\hline KREMEN1 & 224534_PM_at & kringle containing transmembrane & 1.18 & 0.00 & 0.01 \\
\hline CCNYL1 & 227280_PM_s_at & cyclin Y-like 1 & 1.18 & 0.00 & 0.01 \\
\hline PYGL & 202990_PM_at & phosphorylase, glycogen, liver & 1.18 & 0.00 & 0.02 \\
\hline SOX6 & 227498_PM_at & SRY (sex determining region Y)-bo) & 1.18 & 0.00 & 0.00 \\
\hline $\mathrm{CNIH} 4$ & 223993_PM_s_at & cornichon homolog 4 (Drosophila) & 1.18 & 0.00 & 0.03 \\
\hline \multicolumn{2}{|c|}{ LOC100509484209122_PM_at } & uncharacterized LOC100509484 & 1.19 & 0.00 & 0.00 \\
\hline \multirow[t]{2}{*}{ NFIL3 } & 203574_PM_at & \multirow[t]{2}{*}{ nuclear factor, interleukin 3 regula } & 1.19 & 0.00 & 0.02 \\
\hline & 217340_PM_at & & 1.19 & 0.00 & 0.03 \\
\hline KIAA0141 & 227056_PM_at & KIAA0141 & 1.19 & 0.00 & 0.01 \\
\hline CAT & 211922_PM_s_at & catalase & 1.19 & 0.00 & 0.03 \\
\hline UBE2J1 & 217825_PM_s_at & ubiquitin-conjugating enzyme E2, 」 & 1.19 & 0.00 & 0.01 \\
\hline TSNAX & 238973_PM_s_at & translin-associated factor $X$ & 1.19 & 0.00 & 0.02 \\
\hline EID1 & 211698_PM_at & EP300 interacting inhibitor of diffe & 1.19 & 0.00 & 0.02 \\
\hline
\end{tabular}




\begin{tabular}{|c|c|c|c|c|c|}
\hline DPH3 & 225200_PM_at & DPH3, KTI11 homolog (S. cerevisia & 1.19 & 0.00 & 0.00 \\
\hline SYT11 & 209197_PM_at & synaptotagmin XI & 1.19 & 0.00 & 0.01 \\
\hline FAM217B & 225313_PM_at & family with sequence similarity $21^{\circ}$ & 1.20 & 0.00 & 0.01 \\
\hline \multirow[t]{3}{*}{ RHOQ } & 212120_PM_at & ras homolog family member $Q$ & 1.20 & 0.00 & 0.01 \\
\hline & 235286_PM_at & & 1.20 & 0.00 & 0.02 \\
\hline & 237563_PM_s_at & & 1.20 & 0.00 & 0.02 \\
\hline UBE2J1 & 222435_PM_s_at & ubiquitin-conjugating enzyme E2, J & 1.20 & 0.00 & 0.01 \\
\hline \multirow[t]{3}{*}{ CEP350 } & 213165_PM_at & centrosomal protein $350 \mathrm{kDa}$ & 1.20 & 0.00 & 0.01 \\
\hline & 239117_PM_at & & 1.20 & 0.00 & 0.01 \\
\hline & 1569568_PM_at & & 1.20 & 0.00 & 0.00 \\
\hline HSD3B7 & 222817_PM_at & hydroxy-delta-5-steroid dehydrog $\epsilon$ & 1.20 & 0.00 & 0.04 \\
\hline CAT & 201432_PM_at & catalase & 1.20 & 0.00 & 0.00 \\
\hline UBL3 & 201535_PM_at & ubiquitin-like 3 & 1.20 & 0.00 & 0.04 \\
\hline \multirow[t]{2}{*}{ KCTD15 } & 222668_PM_at & potassium channel tetramerisatior & 1.21 & 0.00 & 0.01 \\
\hline & 237071_PM_at & & 1.21 & 0.00 & 0.02 \\
\hline RNF141 & 226106_PM_at & ring finger protein 141 & 1.21 & 0.00 & 0.04 \\
\hline CCPG1 & 221156_PM_x_at & cell cycle progression 1 & 1.21 & 0.00 & 0.03 \\
\hline \multirow[t]{3}{*}{ LOC158402 } & 1559067_PM_a_at & Uncharacterized LOC158402 & 1.21 & 0.00 & 0.02 \\
\hline & 240020_PM_at & & 1.21 & 0.00 & 0.01 \\
\hline & 230176_PM_at & & 1.21 & 0.00 & 0.03 \\
\hline S100A8 & 214370_PM_at & S100 calcium binding protein $A 8$ & 1.21 & 0.00 & 0.00 \\
\hline CD36 & 206488_PM_s_at & CD36 molecule (thrombospondin r & 1.21 & 0.00 & 0.01 \\
\hline UBE2J1 & 217823_PM_s_at & ubiquitin-conjugating enzyme $E 2\rfloor$, & 1.21 & 0.00 & 0.03 \\
\hline \multicolumn{2}{|c|}{ LOC100507015 227356 PM at } & uncharacterized LOC100507015 & 1.22 & 0.00 & 0.02 \\
\hline & 236297_PM_at & & 1.22 & 0.00 & 0.04 \\
\hline STXBP5 & 226794_PM_at & syntaxin binding protein 5 (tomos) & 1.22 & 0.00 & 0.01 \\
\hline BHLHE40 & 201170_PM_s_at & basic helix-loop-helix family, meml & 1.22 & 0.00 & 0.00 \\
\hline MPO & 203948_PM_s_at & myeloperoxidase & 1.22 & 0.00 & 0.04 \\
\hline \multirow[t]{2}{*}{ LIN7A } & 227929_PM_at & lin-7 homolog $A$ (C. elegans) & 1.22 & 0.00 & 0.04 \\
\hline & 240673_PM_at & & 1.22 & 0.00 & 0.00 \\
\hline CR1 & 217552_PM_x_at & complement component $(3 \mathrm{~b}$ & 1.22 & 0.00 & 0.01 \\
\hline CHD9 & 212616_PM_at & chromodomain helicase DNA bindi & 1.22 & 0.00 & 0.00 \\
\hline ZNF321P & 1558888_PM_x_at & zinc finger protein 321 , pseudogen & 1.22 & 0.00 & 0.03 \\
\hline \multirow[t]{2}{*}{$\mathrm{CMC1}$} & 228283_PM_at & COX assembly mitochondrial prote & 1.23 & 0.00 & 0.04 \\
\hline & 239294_PM_at & & 1.23 & 0.00 & 0.00 \\
\hline FAM198B & 223204_PM_at & family with sequence similarity $19 i$ & 1.23 & 0.00 & 0.04 \\
\hline PPID & 228469_PM_at & Peptidylprolyl isomerase $D$ & 1.23 & 0.00 & 0.00 \\
\hline ACSL1 & 201963_PM_at & acyl-CoA synthetase long-chain far & 1.23 & 0.00 & 0.00 \\
\hline \multicolumn{2}{|c|}{ LOC100509498227290_PM_at } & uncharacterized LOC100509498 & 1.24 & 0.00 & 0.00 \\
\hline RGS12 & 1555032_PM_at & regulator of G-protein signaling 12 & 1.24 & 0.00 & 0.03 \\
\hline \multirow[t]{2}{*}{$\mathrm{CD} 24$} & 216379_PM_x_cat & CD24 molecule & 1.24 & 0.00 & 0.05 \\
\hline & 243824_PM_at & & 1.24 & 0.00 & 0.03 \\
\hline SNORD89 & 235536_PM_at & small nucleolar RNA, C & 1.24 & 0.00 & 0.01 \\
\hline KCNJ15 & 238428_PM_at & potassium inwardly-rectifying char & 1.24 & 0.00 & 0.04 \\
\hline АСОТ11 & 216103_PM_at & acyl-CoA thioesterase 11 & 1.24 & 0.00 & 0.01 \\
\hline PTGDR2 & 206361_PM_at & prostaglandin D2 receptor 2 & 1.25 & 0.00 & 0.04 \\
\hline HRASLS5 & 231050_PM_at & HRAS-like suppressor family, mem & 1.25 & 0.00 & 0.01 \\
\hline MCTP2 & 220603_PM_s_at & multiple C2 domains, transmembri & 1.25 & 0.00 & 0.00 \\
\hline HCAR3 & 205220_PM_at & hydroxycarboxylic acid receptor 3 & 1.25 & 0.00 & 0.01 \\
\hline ITPR2 & 202660_PM_at & inositol 1,4,5-trisphosphate recept & 1.25 & 0.00 & 0.01 \\
\hline
\end{tabular}




\begin{tabular}{|c|c|c|c|c|c|}
\hline NDFIP2 & 224802_PM_at & Nedd4 family interacting protein 2 & 1.25 & 0.00 & 0.02 \\
\hline TCN1 & 205513_PM_at & transcobalamin I (vitamin B12 binc & 1.25 & 0.00 & 0.05 \\
\hline DAPK2 & 215184_PM_at & death-associated protein kinase 2 & 1.25 & 0.00 & 0.02 \\
\hline HNMT & 204112_PM_s_at & histamine $\mathrm{N}$-methyltransferase & 1.25 & 0.00 & 0.05 \\
\hline TRPM6 & 240389_PM_at & Transient receptor potential catior & 1.25 & 0.00 & 0.01 \\
\hline FAM120A & 1555947_PM_at & family with sequence similarity 121 & 1.25 & 0.00 & 0.00 \\
\hline MSRB3 & 225782_PM_at & methionine sulfoxide reductase $B 3$ & 1.26 & 0.00 & 0.02 \\
\hline KIF1B & 225878_PM_at & kinesin family member $1 \mathrm{~B}$ & 1.26 & 0.00 & 0.01 \\
\hline CD24 & 209772_PM_s_at & CD24 molecule & 1.26 & 0.00 & 0.02 \\
\hline RPS27L & 218007_PM_s_at & ribosomal protein S27-like & 1.26 & 0.00 & 0.05 \\
\hline PIK3R6 & 1558770_PM_a_at & phosphoinositide-3-kinase, regulat & 1.26 & 0.00 & 0.01 \\
\hline IGHA1 & 1558438_PM_a_at & Immunoglobulin heavy constant al & 1.27 & 0.00 & 0.03 \\
\hline LGALS12 & 223828_PM_s_at & lectin, galactoside-binding, soluble & 1.27 & 0.00 & 0.01 \\
\hline MPO & 203949_PM_at & myeloperoxidase & 1.27 & 0.00 & 0.02 \\
\hline GPR34 & 223620_PM_at & G protein-coupled receptor 34 & 1.28 & 0.00 & 0.03 \\
\hline PNPLA1 & 1553364_PM_at & patatin-like phospholipase domain & 1.28 & 0.00 & 0.00 \\
\hline \multirow[t]{2}{*}{ SLC29A1 } & 201801_PM_s_at & solute carrier family 29 (nucleosidt & 1.28 & 0.00 & 0.00 \\
\hline & 1567100_PM_at & & 1.28 & 0.00 & 0.02 \\
\hline SGMS1 & 212989_PM_at & sphingomyelin synthase 1 & 1.28 & 0.00 & 0.02 \\
\hline CD36 & 228766_PM_at & CD36 molecule (thrombospondin $r$ & 1.28 & 0.00 & 0.00 \\
\hline ARSB & 232197_PM_x_at & arylsulfatase B & 1.28 & 0.00 & 0.00 \\
\hline CYP1B1-AS1 & 1553829_PM_at & CYP1B1 antisense RNA 1 (non-prot & 1.28 & 0.00 & 0.01 \\
\hline \multirow[t]{2}{*}{ CARD16 } & 1552701_PM_a_at & caspase recruitment domain famil & 1.28 & 0.00 & 0.01 \\
\hline & 227051_PM_at & & 1.28 & 0.00 & 0.05 \\
\hline S100A12 & 205863_PM_at & S100 calcium binding protein $\mathrm{A} 12$ & 1.28 & 0.00 & 0.02 \\
\hline C19orf59 & 235568_PM_at & chromosome 19 open reading fran & 1.28 & 0.00 & 0.01 \\
\hline CYP4F12 & 206539_PM_s_at & cytochrome P450, family 4 , subfan & 1.29 & 0.00 & 0.00 \\
\hline ITGA2B & 206493_PM_at & integrin, alpha $2 \mathrm{~b}$ (platelet glycopr & 1.29 & 0.00 & 0.03 \\
\hline PALLD & 200897_PM_s_at & palladin, cytoskeletal associated pı & 1.29 & 0.00 & 0.00 \\
\hline SLC24A3 & 57588_PM_at & solute carrier family 24 (sodium & 1.30 & 0.00 & 0.00 \\
\hline FAM124B & 220637_PM_at & family with sequence similarity 12 & 1.30 & 0.00 & 0.01 \\
\hline ASRGL1 & 222764_PM_at & asparaginase like 1 & 1.30 & 0.00 & 0.00 \\
\hline CAMP & 210244_PM_at & cathelicidin antimicrobial peptide & 1.30 & 0.00 & 0.02 \\
\hline MMP8 & 207329_PM_at & matrix metallopeptidase 8 (neutro & 1.31 & 0.00 & 0.03 \\
\hline F5 & 204714_PM_s_at & coagulation factor V (proaccelerin, & 1.31 & 0.00 & 0.00 \\
\hline MYCT1 & 231947_PM_at & myc target 1 & 1.31 & 0.00 & 0.01 \\
\hline GAPT & 1552386_PM_at & GRB2-binding adaptor protein, tral & 1.31 & 0.00 & 0.00 \\
\hline \multirow[t]{2}{*}{ SLC26A8 } & 237340_PM_at & solute carrier family 26 , member 8 & 1.31 & 0.00 & 0.03 \\
\hline & 243252_PM_at & & 1.31 & 0.00 & 0.04 \\
\hline ZNF321P & 1558887_PM_at & zinc finger protein 321 , pseudogen & 1.32 & 0.00 & 0.01 \\
\hline LPAR1 & 204036_PM_at & lysophosphatidic acid receptor 1 & 1.32 & 0.00 & 0.03 \\
\hline CD24 & 266_PM_s_at & CD24 molecule & 1.32 & 0.00 & 0.01 \\
\hline DACH1 & 228915_PM_at & dachshund homolog 1 (Drosophila & 1.33 & 0.00 & 0.02 \\
\hline F5 & 231029_PM_at & coagulation factor $\mathrm{V}$ (proaccelerin, & 1.34 & 0.00 & 0.00 \\
\hline CTSG & 205653_PM_at & cathepsin G & 1.34 & 0.00 & 0.05 \\
\hline PPBP & 214146_PM_s_at & pro-platelet basic protein (chemok & 1.34 & 0.00 & 0.01 \\
\hline SLC29A1 & 201802_PM_at & solute carrier family 29 (nucleosidt & 1.35 & 0.00 & 0.01 \\
\hline OLIG1 & 228170_PM_at & oligodendrocyte transcription factı & 1.35 & 0.00 & 0.02 \\
\hline LINC00537 & 232034_PM_at & Long intergenic non-protein codin & 1.35 & 0.00 & 0.00 \\
\hline CYP1B1 & 202437_PM_s_at & cytochrome P450, family 1 , subfan & 1.37 & 0.00 & 0.01 \\
\hline
\end{tabular}




\begin{tabular}{|c|c|c|c|c|c|}
\hline COX7B & 202110_PM_at & cytochrome c oxidase subunit VIIb & 1.37 & 0.00 & 0.05 \\
\hline IDO1 & 210029_PM_at & indoleamine 2,3-dioxygenase 1 & 1.37 & 0.00 & 0.03 \\
\hline SMPD3 & 219695_PM_at & sphingomyelin phosphodiesterase & 1.38 & 0.00 & 0.00 \\
\hline VSTM1 & 235818_PM_at & V-set and transmembrane domain & 1.38 & 0.00 & 0.00 \\
\hline HP & 208470_PM_s_at & haptoglobin & 1.38 & 0.00 & 0.00 \\
\hline ARG1 & 206177_PM_s_at & arginase, liver & 1.39 & 0.00 & 0.05 \\
\hline CEBPE & 214523_PM_at & CCAAT & 1.39 & 0.00 & 0.00 \\
\hline SLPI & 203021_PM_at & secretory leukocyte peptidase inhi & 1.40 & 0.00 & 0.00 \\
\hline ANXA3 & 209369_PM_at & annexin A3 & 1.41 & 0.00 & 0.00 \\
\hline ADORA3 & 206171_PM_at & adenosine $\mathrm{A} 3$ receptor & 1.42 & 0.00 & 0.00 \\
\hline CLC & 206207_PM_at & Charcot-Leyden crystal protein & 1.44 & 0.00 & 0.00 \\
\hline CEACAM6 & 203757_PM_s_at & carcinoembryonic antigen-related & 1.45 & 0.00 & 0.01 \\
\hline IL5RA & 210744_PM_s_at & interleukin 5 receptor, alpha & 1.46 & 0.00 & 0.01 \\
\hline CD24 & 208650_PM_s_at & CD24 molecule & 1.46 & 0.00 & 0.00 \\
\hline OLIG2 & 213825_PM_at & oligodendrocyte lineage transcript & 1.47 & 0.00 & 0.01 \\
\hline \multirow[t]{2}{*}{ DEFA1 } & 205033_PM_s_at & defensin, alpha 1 & 1.47 & 0.00 & 0.00 \\
\hline & 240131_PM_at & & 1.47 & 0.00 & 0.00 \\
\hline HP & 206697_PM_s_at & haptoglobin & 1.48 & 0.00 & 0.00 \\
\hline ORM1 & 205040_PM_at & orosomucoid 1 & 1.48 & 0.00 & 0.03 \\
\hline ORM1 & 205041_PM_s_at & orosomucoid 1 & 1.51 & 0.00 & 0.02 \\
\hline CEACAM6 & 211657_PM_at & carcinoembryonic antigen-related & 1.51 & 0.00 & 0.00 \\
\hline RNASE2 & 206111_PM_at & ribonuclease, RNase A family, 2 (lii & 1.52 & 0.00 & 0.00 \\
\hline SIGLEC8 & 208253_PM_at & sialic acid binding Ig-like lectin 8 & 1.52 & 0.00 & 0.00 \\
\hline CRISP3 & 207802_PM_at & cysteine-rich secretory protein 3 & 1.52 & 0.00 & 0.00 \\
\hline LCN2 & 212531_PM_at & lipocalin 2 & 1.53 & 0.00 & 0.01 \\
\hline PRSS33 & 1552349_PM_a_at & protease, serine, 33 & 1.53 & 0.00 & 0.00 \\
\hline BPI & 205557_PM_at & bactericidal & 1.57 & 0.00 & 0.01 \\
\hline IL5RA & 211517_PM_s_at & interleukin 5 receptor, alpha & 1.57 & 0.00 & 0.00 \\
\hline MMP8 & 231688_PM_at & matrix metallopeptidase 8 (neutro & 1.58 & 0.00 & 0.01 \\
\hline TMEM176A & 218345_PM_at & transmembrane protein $176 \mathrm{~A}$ & 1.59 & 0.00 & 0.03 \\
\hline LTF & 202018_PM_s_at & lactotransferrin & 1.61 & 0.00 & 0.02 \\
\hline PRSS33 & 1552348_PM_at & protease, serine, 33 & 1.63 & 0.00 & 0.00 \\
\hline OLFM4 & 212768_PM_s_s_at & olfactomedin 4 & 1.71 & 0.00 & 0.02 \\
\hline CEACAM 8 & 206676_PM_at & carcinoembryonic antigen-related & 1.73 & 0.00 & 0.00 \\
\hline RNASE3 & 206851_PM_at & ribonuclease, RNase A family, 3 & 1.75 & 0.00 & 0.00 \\
\hline CCL23 & 210548_PM_at & chemokine ( $\mathrm{C}-\mathrm{C}$ motif) ligand 23 & 1.75 & 0.00 & 0.00 \\
\hline RNF182 & 230720_PM_at & ring finger protein 182 & 1.81 & 0.00 & 0.01 \\
\hline DEFA4 & 207269_PM_at & defensin, alpha 4 , corticostatin & 1.90 & 0.00 & 0.00 \\
\hline $\mathrm{CCL} 23$ & 210549_PM_s_at & chemokine ( $\mathrm{C}-\mathrm{C}$ motif) ligand 23 & 2.00 & 0.00 & 0.00 \\
\hline
\end{tabular}


U-BIOPRED has been funded by a public-private grant from the Innovative Medicines Initiative (IMI) provided by the European Union (EU) and the European Federation of Pharmaceutical Industries and Associations. The members of the U-BIOPRED Study Group are as follows:

Adcock lan, National Heart and Lung Institute, Imperial College, London, UK;

Adriaens Nora, Academic Medical Centre, University of Amsterdam, The Netherlands;

Ahmed Hassan, European Institute for Systems Biology and Medicine, CNRS-ENS-UCBL-INSERM, Lyon, France;

Aliprantis Antonios, Merck Research Laboratories, Boston, USA;

Alving Kjell, Dept Women's \& Children's Health, Uppsala University, Sweden

Auffray Charles, European Institute for Systems Biology and Medicine, CNRS-ENS-UCBL-INSERM, Lyon, France;

Badorrek Philipp, Fraunhofer ITEM; Cornelia Faulenbach, Fraunhofer ITEM;

Bakke Per, Department of Clinical Science, University of Bergen, Bergen, Norway;

Balgoma David, Centre for Allergy Research, Karolinska Institutet, Stockholm, Sweden

Bansal Aruna T., Acclarogen Ltd, St. John's Innovation Centre, Cambrigde, UK;

Barber Clair, NIHR Southampton Respiratory Biomedical Research Unit and Clinical and Experimental Sciences, Southampton, UK;

Baribaud Frédéric, Janssen R\&D, USA;

Bates Steward, Respiratory Therapeutic Unit, GSK, UK;

Bautmans An, MSD, Brussels, BE

Behndig Annelie F., Dept of Public Health and Clinical Medicine, Umeå University, Umeå, Sweden;

Bel Elisabeth, Academic Medical Centre, University of Amsterdam, The Netherlands;

Beleta Jorge, Almirall S.A., Barcelona, Spain;

Berglind Ann, Dept. Women's and Children's Health \& Centre for Allergy Research, Karolinska Institutet, Stockholm, Sweden

Berton Alix, AstraZeneca, Mölndal, Sweden

Bigler Jeannette, Previously at: Amgen Inc.;

Bisgaard Hans, COPSAC, Copenhagen Prospective Studies on Asthma in Childhood, Herlev and Gentofte Hospital, University of Copenhagen, Copenhagen, Denmark

Bochenek Grazyna, II Department of Internal Medicine, Jagiellonian University Medical College, Krakow, Poland; Boedigheimer Michel J., Amgen Inc.;

Bøonnelykke Klaus, COPSAC, Copenhagen Prospective Studies on Asthma in Childhood, Herlev and Gentofte Hospital, University of Copenhagen, Copenhagen, Denmark;

Brandsma Joost, University of Southampton, UK

Braun Armin, Fraunhofer Institute for Toxicology and Experimental Medicine, Hannover, Germany;

Brinkman Paul, Academic Medical Centre, University of Amsterdam, The Netherlands;

Burg Dominic, Centre for Proteomic Research, Institute for Life Sciences, University of Southampton, UK Campagna Davide, Department of Clinical and Experimental Medicine, University of Catania, Italy; 
Carayannopoulos Leon, MSD, USA;

Caruso Massimo, Dept. Clinical and Experimental Medicine, University of Catania, Italy;

Carvalho da Purificação Rocha João Pedro, Royal Brompton and Harefield NHS Foundation Trust, UK;

Chaiboonchoe Amphun, European Institute for Systems Biology and Medicine, CNRS-ENS-UCBL-INSERM, CIRIUMR5308, Lyon, France;

Chaleckis Romanas, Centre of Allergy Research, Karolinska Institutet, Stockholm, Sweden

Chanez Pascal, Assistance publique des Hôpitaux de Marseille - Clinique des bronches, allergies et sommeil, Aix Marseille Université, Marseille France

Chung Kian F., National Heart and Lung Institute, Imperial College, London, UK;

Coleman Courtney, Asthma UK, London, UK;

Compton Chris, Respiratory Therapeutic Unit, GSK, UK

Corfield Julie, Areteva R\&D, Nottingham, UK;

D’Amico Arnaldo, University of Rome 'Tor Vergata', Rome Italy;

Dahlen Barbro, Karolinska University Hospital \& Centre for Allergy Research, Karolinska Institutet, Stockholm, Sweden

Dahlén Sven-Erik, Centre for Allergy Research, Karolinska Institutet, Stockholm, Sweden

De Alba Jorge, Almirall S.A., Barcelona, Spain;

de Boer Pim, Longfonds, Amersfoort, The Netherlands;

De Lepeleire Inge, MSD, Brussels, BE

De Meulder Betrand, European Institute for Systems Biology and Medicine, CNRS-ENS-UCBL-INSERM, Lyon, France;

Dekker Tamara, Academic Medical Centre, University of Amsterdam, The Netherlands;

Delin Ingrid, Centre for Allergy Research, Karolinska Institutet, Stockholm, Sweden

Dennison Patrick, NIHR Southampton Respiratory Biomedical Research Unit, Clinical and Experimental Sciences, NIHR-Wellcome Trust Clinical Research Facility, Faculty of Medicine, University of Southampton, UK;

Dijkhuis Annemiek, Academic Medical Centre, University of Amsterdam, The Netherlands;

Djukanovic Ratko, NIHR Southampton Respiratory Biomedical Research Unit and Clinical and Experimental Sciences, Southampton, UK;

Draper Aleksandra, BioSci Consulting, Maasmechelen, Belgium;

Edwards Jessica, Asthma UK, London, UK;

Emma Rosalia, Department of Clinical and Experimental Medicine, University of Catania, Italy;

Ericsson Magnus, Karolinska University Hospital, Stockholm, Sweden

Erpenbeck Veit, Translational Medicine, Respiratory Profiling, Novartis Institutes for Biomedical Research, Basel, Switzerland;

Erzen Damijan, Boehringer Ingelheim Pharma GmbH \& Co. KG; Biberach, Germany

Fichtner Klaus, Boehringer Ingelheim Pharma GmbH \& Co. KG; Biberach, Germany

Fitch Neil, BioSci Consulting, Maasmechelen, Belgium;

Fleming Louis J., National Heart and Lung Institute, Imperial College, London, UK; 
Flood Breda, Asthma UK, UK;

Fowler Stephen J., Centre for Respiratory Medicine and Allergy, Institute of Inflammation and Repair, University of Manchester and University Hospital of South Manchester, Manchester Academic Health Sciences Centre, Manchester, United Kingdom

Frey Urs, University Children's Hospital, Basel, Switzerland;

Gahlemann Martina, Boehringer Ingelheim (Schweiz) GmbH, Switzerland;

Galffy Gabriella, Semmelweis University, Budapest, Hungary;

Gallart Hector, Centre for Allergy Research, Karolinska Institutet, Stockholm, Sweden

Garrett Trevor, BioSci Consulting, Maasmechelen, Belgium;

Geiser Thomas, Department of Respiratory Medicine, University Hospital Bern, Switzerland;

Gent Julaiha, Royal Brompton and Harefield NHS Foundation Trust, UK;

Gerhardsson de Verdier Maria, AstraZeneca; Molndal, Sweden;

Gibeon David, National Heart and Lung Institute, Imperial College, UK;

Gomez Cristina, Centre for Allergy Research, Karolinska Institutet, Stockholm, Sweden

Gove Kerry, NIHR Southampton Respiratory Biomedical Research Unit and Clinical and Experimental Sciences, Southampton, UK;

Gozzard Neil, UCB, UK;

Guo Yi-ke, Data Science Institute, Imperial College, UK;

Hashimoto Simone, Academic Medical Centre, University of Amsterdam, The Netherlands;

Haughney John, International Primary Care Respiratory Group, Aberdeen, Scotland;

Hedlin Gunilla, Dept. Women's and Children's Health \& Centre for Allergy Research, Karolinska Institutet, Stockholm, Sweden;

Hekking Pieter-Paul, Academic Medical Centre, University of Amsterdam, The Netherlands;

Henriksson Elisabeth, Karolinska University Hospital \& Karolinska Institutet, Stockholm, Sweden

Hewitt Lorraine, NIHR Southampton Respiratory Biomedical Research Unit, Southampton, UK

Higgenbottam Tim, Allergy Therapeutics, West Sussex, UK;

Hoda Uruj, Imperial College, UK;

Hohlfeld Jens, Fraunhofer Institute for Toxicology and Experimental Medicine, Hannover, Germany;

Holweg Cecile, Respiratory and Allergy Diseases, Genentech, San Francisco CA;

Horvath Ildiko, Semmelweis University, Budapest, Hungary;

Howarth Peter, NIHR Southampton Respiratory Biomedical Research Unit, Clinical and Experimental Sciences and Human Development and Health, Southampton, UK;

Hu Richard, Amgen Inc.;

Hu Sile, National Heart and Lung Institute, Imperial College, UK;

Hu Xugang, Amgen Inc.;

Hudson Val, Asthma UK, London, UK;

James Anna J., Centre for Allergy Research, Karolinska Institutet, Stockholm, Sweden;

Kamphuis Juliette, Longfonds, Amersfoort, The Netherlands; 
Kennington Erika J., Asthma UK, London, UK;

Kerry Dyson, CromSource, Stirling UK;

Klüglich Matthias, Boehringer Ingelheim Pharma GmbH \& Co. KG; Biberach, Germany

Knobel Hugo, Philips Research Laboratories, Eindhoven, The Netherlands;

Knowles Richard, Arachos Pharma, UK;

Knox Alan, Respiratory Research Unit, University of Nottingham, UK;

Kolmert Johan, Centre for Allergy Research, Karolinska Institutet, Stockholm, Sweden

Konradsen Jon, Dept. Women's and Children's Health \& Centre for Allergy Research, Karolinska Institutet, Stockholm, Sweden

Kots Maxim, Chiesi Pharmaceuticals, SPA, Parma, Italy;

Krueger Linn, University Children's Hospital Bern, Switzerland

Krug Norbert, Fraunhofer Institute for Toxicology and Experimental Medicine, Hannover, Germany;

Kuo Scott, National Heart and Lung Institute, Imperial College, UK;

Kupczyk Maciej, Centre for Allergy Research, Karolinska Institutet, Stockholm, Sweden

Lambrecht Bart, University of Gent, Gent, Belgium;

Lantz Ann-Sofie, Karolinska University Hospital \& Centre for Allergy Research, Karolinska Institutet, Stockholm, Sweden;

Larsson Lars, AstraZeneca, Mohlndal, Sweden;

Lazarinis Nikos, Karolinska University Hospital \& Karolinska Institutet, Stockholm, Sweden

Lefaudeux Diane, European Institute for Systems Biology and Medicine, CNRS-ENS-UCBL-INSERM, Lyon, France; Lone-Latif Saeeda, Academic Medical Centre, University of Amsterdam, The Netherlands;

Loza Matthew J., Janssen R\&D, USA;

Lutter Rene, Academic Medical Centre, University of Amsterdam, The Netherlands;

Marouzet Lisa, NIHR Southampton Respiratory Biomedical Research Unit, Southampton, UK

Martin Jane, NIHR Southampton Respiratory Biomedical Research Unit, Southampton, UK

Masefield Sarah, European Lung Foundation, Sheffield, UK;

Mathon Caroline, Centre of Allergy Research, Karolinska Institutet, Stockholm, Sweden

Matthews John G., Respiratory and Allergy Diseases, Genentech, San Francisco CA, USA;

Mazein Alexander, European Institute for Systems Biology and Medicine, CNRS-ENS-UCBL-INSERM, CIRI-

UMR5308, Lyon, France;

Meah Sally, National Heart and Lung Institute, Imperial College, UK;

Meiser Andrea, National Heart and Lung Institute, Imperial College, UK;

Menzies-Gow Andrew, Royal Brompton and Harefield NHS Foundation Trust, UK;

Metcalf Leanne, Previously at: Asthma UK, London, UK;

Middelveld Roelinde, Centre for Allergy Research, Karolinska Institutet, Stockholm, Sweden

Mikus Maria, Science for Life Laboratory \& The Royal Institute of Technology, Stockholm, Sweden;

Miralpeix Montse, Almirall, Barcelona, Spain; 
Monk Philip, Synairgen Research Ltd, Southampton, UK;

Montuschi Paolo, Università Cattolica del Sacro Cuore, Italy;

Mores Nadia, Università Cattolica del Sacro Cuore, Italy;

Murray Clare S., Centre for Respiratory Medicine and Allergy, Institute of Inflammation and Repair, University of Manchester and University Hospital of South Manchester, Manchester Academic Health Sciences Centre,

Manchester, United Kingdom

Musial Jacek, II Department of Internal Medicine, Jagiellonian University Medical College, Krakow, Poland;

Myles David, Respiratory Therapeutic Unit, GSK, UK;

Naz Shama, Centre for Allergy Research, Karolinska Institutet, Stockholm, Sweden

Nething Katja, Boehringer Ingelheim Pharma GmbH \& Co. KG; Biberach, Germany

Nicholas Ben, University of Southampton, UK

Nihlen Ulf, AstraZeneca; Molndal, Sweden;

Nilsson Peter, Science for Life Laboratory \& The Royal Institute of Technology, Stockholm, Sweden;

Nordlund Björn, Dept. Women's and Children's Health \& Centre for Allergy Research, Karolinska Institutet, Stockholm, Sweden

Östling Jörgen, AstraZeneca, Mölndal, Sweden;

Pacino Antonio, Lega Italiano Anti Fumo, Catania, Italy;

Pahus Laurie, Assistance publique des Hôpitaux de Marseille, Clinique des bronches, allergies et sommeil Espace Éthique Méditerranéen, Aix-Marseille Université, Marseille, France;

Palkonen Susanna, European Federation of Allergy and Airways Diseases Patient's Associations, Brussels, Belgium.

Pandis loannis, Data Science Institute, Imperial College, London, UK;

Pavlidis Stelios, National Heart and Lung Institute, Imperial College, UK

Pennazza Giorgio, University of Rome 'Tor Vergata', Rome Italy;

Petrén Anne, Centre for Allergy Research, Karolinska Institutet, Stockholm, Sweden

Pink Sandy, NIHR Southampton Respiratory Biomedical Research Unit, Southampton, UK

Postle Anthony, University of Southampton, UK

Powel Pippa, European Lung Foundation, Sheffield, UK;

Rahman-Amin Malayka, Previously at: Asthma UK, London, UK;

Rao Navin, Janssen R\&D, USA;

Ravanetti Lara, Academic Medical Centre, University of Amsterdam, The Netherlands;

Ray Emma, NIHR Southampton Respiratory Biomedical Research Unit, Southampton, UK

Reinke Stacey, Centre for Allergy Research, Karolinska Institutet, Stockholm, Sweden

Reynolds Leanne, Previously at: Asthma UK, London, UK;

Riemann Kathrin, Boehringer Ingelheim Pharma GmbH \& Co. KG; Biberach, Germany

Riley John, Respiratory Therapeutic Unit, GSK, UK;

Robberechts Martine, MSD, Brussels, BE

Roberts Amanda, Asthma UK, UK; 
Roberts Graham, NIHR Southampton Respiratory Biomedical Research Unit, Clinical and Experimental Sciences and Human Development and Health, Southampton, UK;

Rossios Christos, National Heart and Lung Institute, Imperial College, UK;

Rowe Anthony, Janssen R\&D, UK;

Russell Kirsty, National Heart and Lung Institute, Imperial College, UK;

Rutgers Michael, Longfonds, Amersfoort, The Netherlands;

Sandström Thomas, Dept of Public Health and Clinical Medicine, Umeå University, Umeå, Sweden;

Santini Giuseppe, Università Cattolica del Sacro Cuore, Italy;

Santoninco Marco, University of Rome 'Tor Vergata', Rome Italy;

Schoelch Corinna, Boehringer Ingelheim Pharma GmbH \& Co. KG, Biberach, Germany

Schofield James P. R., Centre for Proteomic Research, Institute for Life Sciences, University of Southampton, UK

Seibold Wolfgang, Boehringer Ingelheim Pharma GmbH, Biberach, Germany

Shaw Dominick E., Respiratory Research Unit, University of Nottingham, UK;

Sigmund Ralf, Boehringer Ingelheim Pharma GmbH \& Co. KG; Biberach, Germany

Singer Florian, University Children's Hospital, Zurich, Switzerland;

Sjödin Marcus, Centre for Allergy Research, Karolinska Institutet, Stockholm, Sweden

Skipp Paul J., Centre for Proteomic Research, Institute for Life Sciences, University of Southampton, UK

Smids Barbara, Academic Medical Centre, University of Amsterdam, The Netherlands;

Smith Caroline, NIHR Southampton Respiratory Biomedical Research Unit, Southampton, UK

Smith Jessica, Asthma UK, London, UK;

Smith Katherine M., University of Nottingham, UK;

Söderman Päivi, Dept. Women's and Children's Health, Karolinska Institutet, Stockholm, Sweden

Sogbesan Adesimbo, Royal Brompton and Harefield NHS Foundation Trust, UK;

Sousa Ana R., Respiratory Therapeutic Unit, GSK, UK;

Staykova Doroteya, University of Southampton, UK

Sterk Peter J., Academic Medical Centre, University of Amsterdam, The Netherlands;

Strandberg Karin, Karolinska University Hospital \& Karolinska Institutet, Stockholm, Sweden

Sun Kai, National Heart and Lung Institute, Imperial College, UK;

Supple David, Asthma UK, UK;

Szentkereszty Marton, Semmelweis University, Budapest, Hungary;

Tamasi Lilla, Semmelweis University, Budapest, Hungary;

Tariq Kamran, NIHR Southampton Respiratory Biomedical Research Unit, Clinical and Experimental Sciences, NIHR-Wellcome Trust Clinical Research Facility, Faculty of Medicine, University of Southampton, UK;

Thörngren John-Olof, Karolinska University Hospital, Sweden

Thornton Bob, MSD, USA;

Thorsen Jonathan, COPSAC, Copenhagen Prospective Studies on Asthma in Childhood, Herlev and Gentofte Hospital, University of Copenhagen, Copenhagen, Denmark; 
Valente Salvatore, Università Cattolica del Sacro Cuore, Italy;

van Aalderen Wim, Academic Medical Centre, University of Amsterdam, The Netherlands;

van de Pol Marianne, Academic Medical Centre, University of Amsterdam, The Netherlands;

van Drunen Kees, Academic Medical Centre, University of Amsterdam, The Netherlands;

van Geest Marleen, AstraZeneca, Mölndal, Sweden;

Versnel Jenny, Previously at: Asthma UK, London, UK;

Vestbo Jorgen, Centre for Respiratory Medicine and Allergy, Institute of Inflammation and Repair, University of Manchester and University Hospital of South Manchester, Manchester Academic Health Sciences Centre, Manchester, United Kingdom

Vink Anton, Philips Research Laboratories, Eindhoven, The Netherlands;

Vissing Nadja, COPSAC, Copenhagen Prospective Studies on Asthma in Childhood, Herlev and Gentofte Hospital, University of Copenhagen, Copenhagen, Denmark;

von Garnier Christophe, University Hospital Bern, Switzerland;

Wagener Ariane, Academic Medical Centre, University of Amsterdam, The Netherlands;

Wagers Scott, BioSci Consulting, Maasmechelen, Belgium;

Wald Frans, Boehringer Ingelheim Pharma GmbH \& Co. KG, Biberach, Germany

Walker Samantha, Asthma UK, London, UK;

Ward Jonathan, Histochemistry Research Unit, Faculty of Medicine, University of Southampton, Southampton, UK;

Weiszhart Zsoka, Semmelweis University, Budapest, Hungary;

Wetzel Kristiane, Boehringer Ingelheim Pharma GmbH, Biberach, Germany

Wheelock Craig E., Centre for Allergy Research, Karolinska Institutet, Stockholm, Sweden;

Wiegman Coen, National Heart and Lung Institute, Imperial College, UK;

Williams Siân, International Primary Care Respiratory Group, Aberdeen, Scotland;

Wilson Susan J., Histochemistry Research Unit, Faculty of Medicine, University of Southampton, Southampton, UK;

Woodcock Ashley, Centre for Respiratory Medicine and Allergy, Institute of Inflammation and Repair, University of Manchester and University Hospital of South Manchester, Manchester Academic Health Sciences Centre, Manchester, United Kingdom

Yang Xian, National Heart and Lung Institute, Imperial College, UK;

Yeyasingham Elizabeth, UK Clinical Operations, GSK, Stockley Park, UK;

Yu Wen, Amgen Inc.;

Zetterquist Wilhelm, Dept. Women's and Children's Health \& Centre for Allergy Research, Karolinska Institutet, Stockholm, Sweden

Zwinderman Koos, Academic Medical Centre, University of Amsterdam, The Netherlands; 